\title{
NEUTRON SCATTERING AND MICROSCOPY STUDIES OF THE STRUCTURE AND DYNAMICS OF WATER NEAR A NANOSTRUCTURED HYDROPHILIC COPPER OXIDE SURFACE
}

\author{
A Dissertation presented to \\ the Faculty of the Graduate School \\ at the University of Missouri-Columbia \\ In Partial Fulfillment \\ of the Requirements for the Degree \\ Doctor of Philosophy \\ by \\ JAMES R. TORRES \\ Professor Haskell Taub, Dissertation Supervisor
}

July 2019 
(C) Copyright by James Torres 2019

All Rights Reserved 
The undersigned, appointed by the Dean of the Graduate School, have examined the dissertation entitled:

NEUTRON SCATTERING AND MICROSCOPY STUDIES OF THE STRUCTURE AND DYNAMICS OF WATER NEAR A NANOSTRUCTURED HYDROPHILIC COPPER OXIDE SURFACE

presented by James Torres, a candidate for the degree of Doctor of Philosophy and hereby certify that, in their opinion, it is worthy of acceptance.

Dr. Haskell Taub

Dr. Helmut Kaiser

Dr. Paul Miceli

Dr. Robert A. Winholtz

Dr. Tommi White

Dr. Kenneth W. Herwig 


\section{Acknowledgements}

There are a great number of people that contributed to this body of work without whom this thesis could not have been possible. I would like to thank my thesis adviser, Prof. Haskell Taub, for preparing me for a career in experimental scientific research through his guidance, patience, and dedication to my success. I thank my reactor adviser, Dr. Helmut Kaiser, for taking every opportunity to engage my interests in neutron scattering and instrumentation at MURR. My education and hands-on experience in neutron scattering as well as my attendance at numerous conferences and workshops were made possible by the support from the NSF-funded Integrative Graduate Education and Research Traineeship (IGERT) program at MU. To the experts at the MU Electron Microscopy Core, I thank you for supporting my work in, and now passion for, electron microscopy that I carry forward. I also thank our collaborators, Drs. Robert Winholtz, Tom Zhang and Hongbin Ma, for providing the foundations of this thesis.

I wish to express my gratitude towards Dr. Ken Herwig for his championship of our research during my internship in the Graduate Opportunity (GO!) program at Oak Ridge National Laboratory. This invaluable experience has had a positive impact on my career goals in STEM research as well as my approach to new challenges.

I also appreciate the support from the MU Department of Physics and Astronomy and my fellow graduate students. Many thanks to Alessandro Mazza for being an excellent friend and colleague. To Zack Buck, thank you for your help in conducting experiments and performing data analysis during my first years as a graduate student. Best of luck to you both. And to Tiffanie, who was there from the beginning, thank you for sharing your immense love and friendship, especially during these last few months. 


\section{Table of Contents}

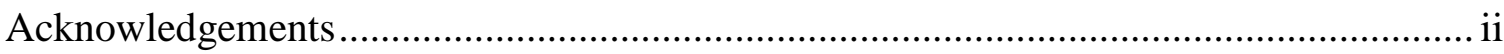

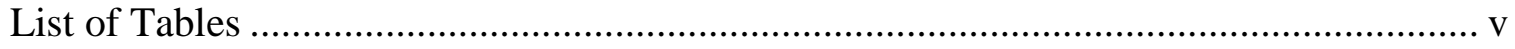

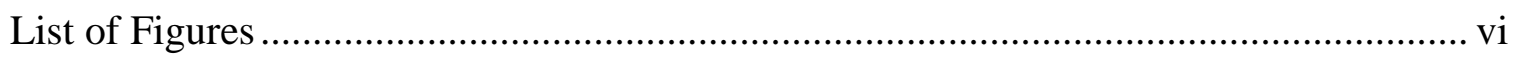

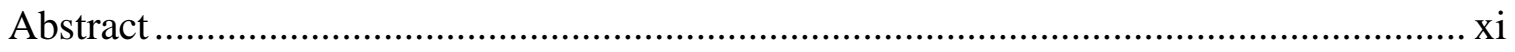

Chapter 1 Introduction to Oscillating Heat Pipes and Hydrophilic Materials .................. 1

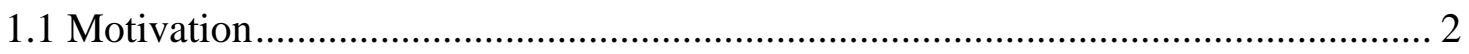

1.2 Development of Oscillating Heat Pipes (OHPs) .................................................. 3

1.3 Surface Wetting, Superhydrophilicity, and the Unusual Properties of Interfacial and

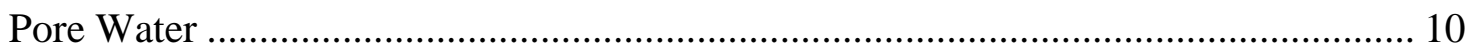

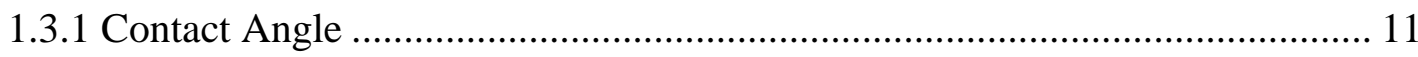

1.3.2 Review of Interfacial and Confined Water .................................................... 14

Chapter 2 The Structure, Morphology, and Wetting Properties of Nanostructured CuO. 22

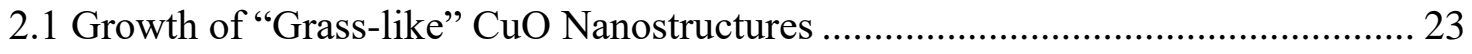

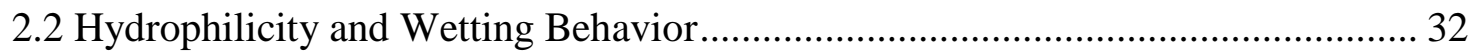

2.3 Surface Area, Porosity, and Sorption Properties of the Nanostructured $\mathrm{CuO}$

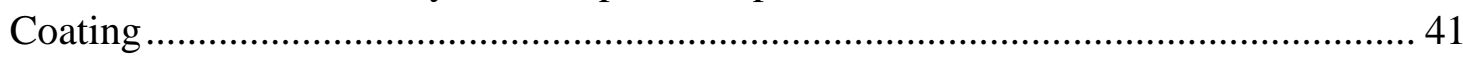

Chapter 3 Structure and Freezing/Melting Behavior of Water Near Hydrophilic CuO

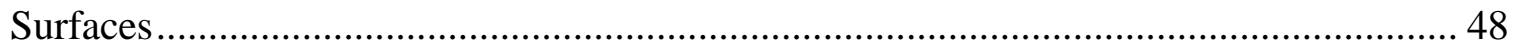

3.1 Two Complementary Backscattering Neutron Spectrometers............................. 49

3.2 Preparation of Samples for Neutron Scattering ................................................ 52

3.3 Elastic "Fixed-Window" Scans Measured on the HFBS ...................................... 53

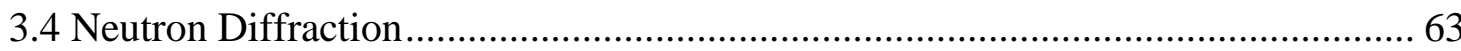

Chapter 4 Dynamics of Interfacial Water Near Hydrophilic CuO-Nanostructured

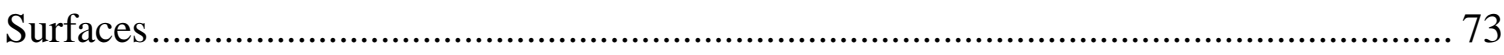

4.1 Inelastic Neutron Scattering from Water Near Hydrophilic CuO-Nanostructured

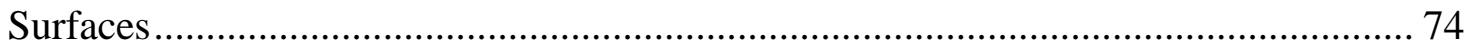

4.2 Quasielastic Neutron Scattering from Water Near Hydrophilic CuO-

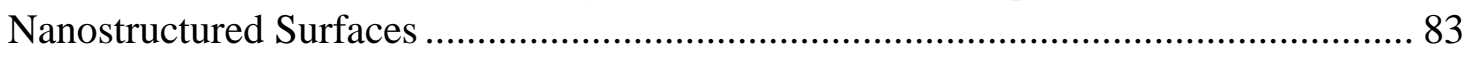

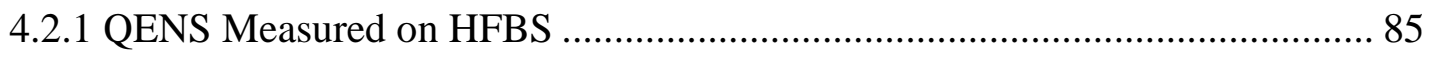

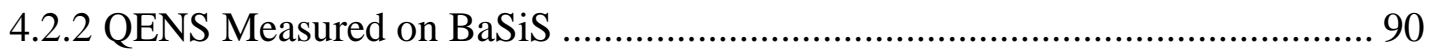

4.2.3 Comparing HFBS and BaSiS Elastic Intensities ....................................... 92

4.2.4 Motions Occurring on Two Time Scales .................................................. 95 


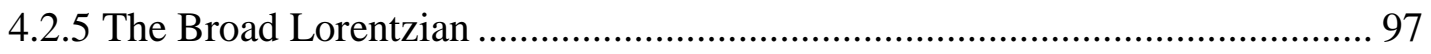

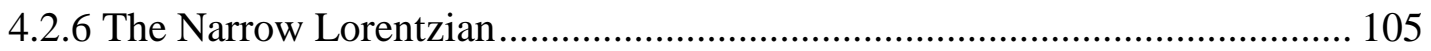

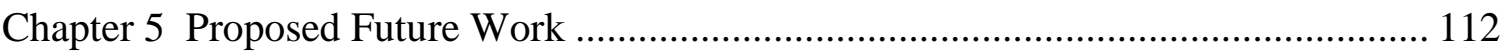

5.1 Time-Resolved Neutron Imaging of Liquid Water Dynamics in a CuO-Coated

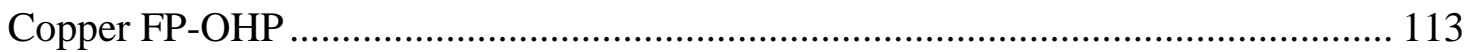

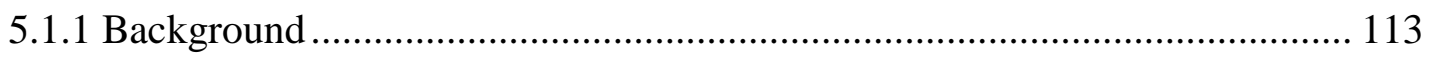

5.1.2 Thermal Performance Measurements .......................................................... 114

5.1.3 Neutron Imaging Measurements ................................................................... 116

5.2 Conceptual Design of a Thermal Neutron Imaging Facility at the MU Research

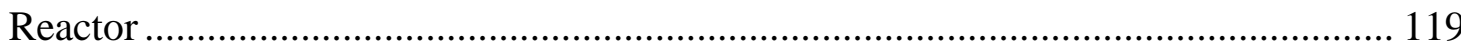

5.2.1 MURR Design and Performance …………………….............................. 120

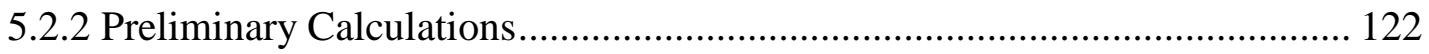

5.2.3 Instrument Layout ................................................................................... 125

5.2.4 Neutron and Gamma-Ray Filters and Shielding ........................................... 131

5.2.5 CAD Drawings of the MURR-NIS ........................................................... 140

5.2.6 McStas Neutron Ray-Tracing Computer Simulations ................................... 144

Appendix A - Samples and Sample Cell Used for Neutron Scattering.......................... 148

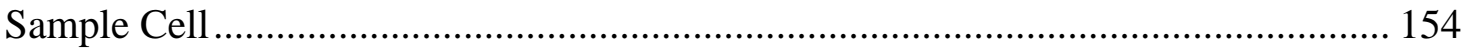

Appendix B - Estimating the Amount of Frozen and/or Immobile Water in HFBS Elastic

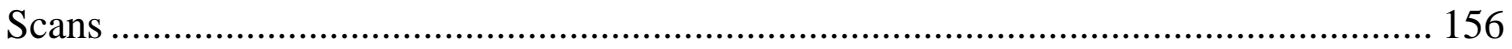

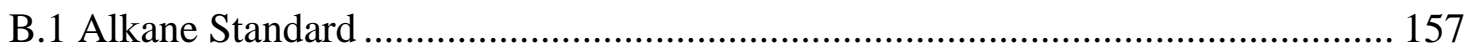

B.2 Water-Film Thickness on $\mathrm{Cu}$ and $\mathrm{CuO}$ Surfaces …….................................... 160 Appendix C - Neutron Diffraction Bragg Peak Analysis at the MU Research Reactor 166

C.1 Temperature Dependence of the Diffraction Patterns.......................................... 166

C.2 Correction to Bragg Peak Position ................................................................ 170

C.3 $\mathrm{D}_{2} \mathrm{O}$ Ice Particle Domain Size Calculation ...................................................... 175

Appendix D - McStas Script Used to Simulate Instrument Performance ....................... 177

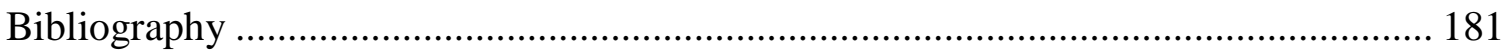

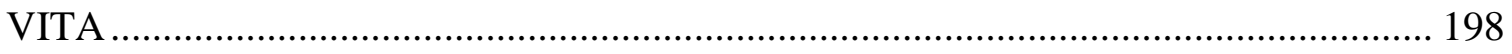




\section{List of Tables}

Table 2.1. Comparison between observed and calculated $d$-spacings for Bragg spots indexed from SAED patterns of CuO-coated samples

Table 3.1. Approximate wave vector transfer $(Q)$ ranges, energy-transfer (dynamic) ranges, and energy resolutions of the HFBS and BaSiS instruments used in QENS experiments

Table 3.2. Calculated and observed Bragg peak positions of $\mathrm{Cu}, \mathrm{Cu}_{2} \mathrm{O}$, and $\mathrm{CuO}$ phases in the diffraction pattern of a non-hydrated $\mathrm{CuO}$-coated sample .....

Table 4.1. Various types of amorphous ice produced experimentally 82

Table 5.1. Exemplary neutron energy ranges 120

Table 5.2. Instrument settings and performance 125

Table 5.3. Empirical Debye temperatures of several crystalline materials 132

Table 5.4. Estimations of the radiation shielding's dimensions, weight, and cost 139

Table 5.5. Results of neutron ray-tracing simulations

Table A.1. List of samples, their composition, and the period in which they were used in neutron scattering experiments, between May of 2015 and August of 2018.

Table B.1. Coherent $\left(\sigma_{\text {coh }}\right)$ and incoherent scattering $\left(\sigma_{\text {inc }}\right)$ cross sections of the elements in the alkane standard sample 158

Table B.2. Monitor-normalized elastic intensity $(I(T))$ measured at the minimum $\left(T_{\min }\right)$ and maximum $\left(T_{\max }\right)$ temperatures of the elastic scans shown in Fig. B.2 163

Table C.1. $\mathrm{D}_{2} \mathrm{O}$ peak position corrected for instrument effects using $\mathrm{Cu}(111)$ fiducial. 171

Table C.2. $\mathrm{D}_{2} \mathrm{O}$ peak position corrected for instrument effects using $\mathrm{CuO}(111)$ fiducial

Table C.3. Width of the $\mathrm{D}_{2} \mathrm{O}(100)$ peak, measured in degrees, which is converted to domain size 


\section{List of Figures}

Figure 1.1. Illustration of a conventional heat pipe (CHP) ........................................ 3

Figure 1.2. Exploded view of a notebook computer with a copper heat pipe integrated in

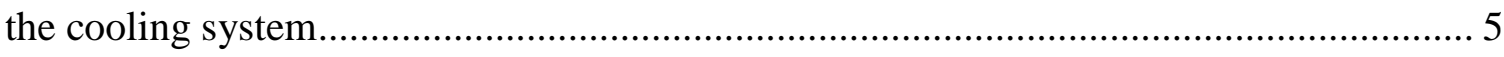

Figure 1.3. (a) Diagram and (b) image of a closed-loop OHP consisting of a narrow capillary tube meandering between a heat source (evaporator) and heat sink (condenser). 6

Figure 1.4. SEM image of $\mathrm{CuO}$ nanostructures grown on copper via hot alkaline bath .... 7

Figure 1.5. Schematic diagram of a water contact angle $\theta$ measured at the contact line formed at the intersection of three phases: solid substrate, liquid water droplet, and

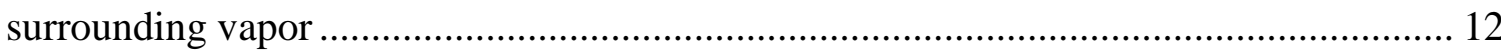

Figure 1.6. Generalized continuum models of interfacial and bulk water ...................... 15

Figure 2.1. Photos of a 5-cm diameter, 12- $\mu \mathrm{m}$ thick copper foil (left) before and (right)

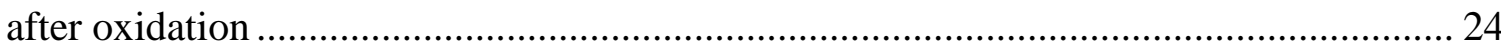

Figure 2.2. SEM image of a discolored "grey" patch on the oxidized copper foil .......... 25

Figure 2.3. Energy-dispersive X-ray spectroscopy (EDS) scans of (a) black and (b) discolored regions of copper foil oxidized in an alkaline solution ................................. 26

Figure 2.4. SEM images of a copper surface exposed to an oxidizing solution .............. 28

Figure 2.5. SEM image of the grass-like morphology of $\mathrm{CuO}$ nanostructures................. 28

Figure 2.6. SEM image of a cross section of the sample used for TEM characterization 29

Figure 2.7. (a) HAADF image of a cross section of a chemically oxidized $\mathrm{Cu}$ foil. Layers $\left(\mathrm{Cu}, \mathrm{Cu}_{2} \mathrm{O}\right.$, and $\left.\mathrm{CuO}\right)$ are identified based on image contrast, enabling the $\mathrm{Cu}_{2} \mathrm{O}$ region to be outlined by the dashed red curve. (b) SAED pattern taken in the circled region in (a) along the [112] zone axis of $\mathrm{Cu}$ and $\mathrm{Cu}_{2} \mathrm{O}$. (c) EELS scans taken within each layer of (a)

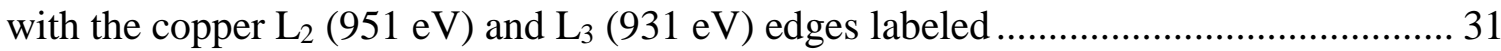

Figure 2.8. Equilibrium water contact-angle measurements, using $1 \mu 1$ droplets, for (a) bare- $\mathrm{Cu}$ and (b) $\mathrm{CuO}$-coated $\mathrm{Cu}$ surfaces with average values of $70^{\circ}$ and $\sim 0^{\circ}$, respectively. (c-d) Observation by ESEM of water condensation on (c) untreated-Cu and

(d) $\mathrm{CuO}$ surfaces 33

Figure 2.9. ESEM image of water condensed on the surface of a bare-Cu foil............... 36

Figure 2.10. Equilibrium phase diagram of bulk water ........................................... 37 
Figure 2.11. False-colored ESEM images of water actively condensing on bare $\mathrm{Cu}$ (a-c) and $\mathrm{CuO}$ nanostructures (d-f).

Figure 2.12. Magnified ESEM images of the dry (a) and wet (b-c) CuO surfaces .......... 39

Figure 2.13. ESEM video screenshots of water condensing on a $\mathrm{CuO}$-coated $\mathrm{Cu}$ foil at multiple length scales.

Figure 2.14. (a) Vapor isotherms for adsorption (closed squares) and desorption (open circles) of $\mathrm{N}_{2}$ as a function of reduced pressure $\left(P / P_{0}\right)$ at $77 \mathrm{~K}$. (b) Plot of the cumulative amount of $\mathrm{N}_{2}$ adsorbed by $\mathrm{CuO}$-coated samples vs. pore diameter................................. 43

Figure 2.15. Water vapor adsorption and desorption isotherms measured at 298 K........ 45

Figure 2.16. Topographical AFM image of a $10 \times 10 \mu \mathrm{m}^{2}$ region of a nanostructured $\mathrm{CuO}$ surface

Figure 3.1. Schematic of the HFBS showing the major components of the instrument... 52

Figure 3.2. Sketch of the sample cell used for neutron scattering experiments................ 53

Figure 3.3. Elastic scans measured on the HFBS vs. sample temperature of three samples

Figure 3.4. Mean-squared displacement (MSD, $\left\langle u^{2}\right\rangle$ ) plotted vs. temperature on cooling at a constant ramp rate of $0.08 \mathrm{~K} / \mathrm{min}$ for the wet (blue) and dry (red) $\mathrm{CuO}$-coated samples

Figure 3.5. Photo of the two-axis powder neutron diffractometer located at D-port at MURR

Figure 3.6. Semi-log plot of the diffraction intensity for a CuO-coated sample at $\sim 300 \mathrm{~K}$

Figure 3.7. Neutron diffraction scans at $\sim 240 \mathrm{~K}$ vs. wave vector transfer $Q$ for four samples.

Figure 3.8. (a) Diffraction scans of the $\mathrm{CuO}$ sample hydrated with $240 \mu 1$ of $\mathrm{D}_{2} \mathrm{O}$ as a function of temperature taken every $5 \mathrm{~K}$ on cooling from $295 \mathrm{~K}$ to $200 \mathrm{~K}$. (b) Temperature dependence of the $\mathrm{D}_{2} \mathrm{O}(100)$ Bragg peak integrated intensity normalized to the peak at $260 \mathrm{~K}$ determined from a Gaussian fit to the peaks in (a).

Figure 4.1. Inelastic neutron scattering (INS) spectra of bulk water at various temperatures at low energy-transfers.

Figure 4.2. Monitor-normalized vibrational spectra measured at $5 \mathrm{~K}$ in the low energytransfer range for our four samples

Figure 4.3. Vibrational spectra reproduced from Fig. 4.2 and now normalized to the copper phonon centered around $3 \mathrm{meV}$. 
Figure 4.4. Difference spectra at $5 \mathrm{~K}$ of the wet (blue and green) and dry (purple) samples with the spectrum of the vacuum dried $\mathrm{CuO}$ sample $(\mathrm{CuO}+0 \mu \mathrm{l})$ subtracted. 79

Figure 4.5. FWS reproduced from Fig. 3.3 excluding the detector at $Q=0.25 \AA^{-1}$ in order to reduce the coherent scattering from $\mathrm{Cu}$ the substrate. 84

Figure 4.6. QENS spectrum of the wet CuO sample at $Q \sim 0.7 \AA^{-1}$ measured on the HFBS

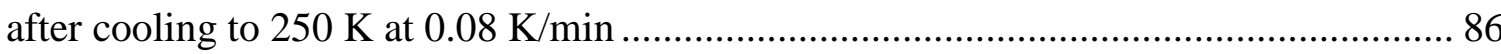

Figure 4.7. HWHM $\Gamma$ of the quasielastic peak vs. $Q^{2}$ for bulk water from Ref. $161 \ldots . . .88$

Figure 4.8. HWHM of the single Lorentzian function fit to QENS spectra of the wet $\mathrm{CuO}$

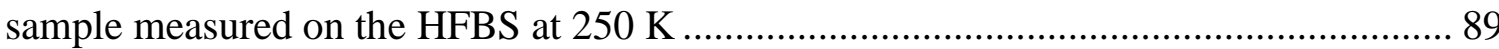

Figure 4.9. QENS spectrum of the wet CuO-coated sample measured on $\mathrm{BaSiS}$ at $Q=1.1$

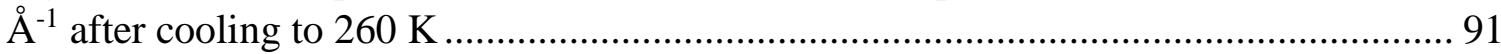

Figure 4.10. (a) Elastic intensity vs. temperature measured on HFBS (blue and orange points) and $\mathrm{BaSiS}$ (red and green points) for the wet $\mathrm{CuO}$ (squares) and dry (circles) $\mathrm{CuO}$-coated samples. (b) Integrated intensities of the quasielastic components (narrow plus broad Lorentzians) measured on BaSiS as a function of temperature .................... 93

Figure 4.11. Intensity of the background term $b Q$ in the linear background intensity $V(Q, \omega)=a(Q) \omega+b(Q)$ plotted versus $Q$ for the wet (closed points) and dry (open points) samples at all temperatures measured on BaSiS.

Figure 4.12. Temperature dependence of the intensity of the narrow and broad Lorentzians in the $\mathrm{BaSiS}$ spectra of the wet and dry $\mathrm{CuO}$-coated samples .....

Figure 4.13. HWHM vs $Q^{2}$ of the broad Lorentzian as measured on BaSiS at 270, 250, and $230 \mathrm{~K}$

Figure 4.14. HWHM of Lorentzian functions fit to QENS spectra of supercooled bulk water at a temperature around $260 \mathrm{~K}$ by various research groups

Figure 4.15. Temperature dependence of the $Q$-weighted intensity of the (a) broad and (b) narrow Lorentzian components in the BaSiS spectra of both the wet and dry CuO-coated samples.....

Figure 4.16. HWHM of the narrow Lorentzian $\Gamma_{N}$ as a function of $Q^{2}$ at selected temperatures as measured on BaSiS for (a) the wet $(60 \mu \mathrm{l})$ and (b) dry (10 $\mu \mathrm{l}) \mathrm{CuO}$ coated samples 106

Figure 4.17. Pressure-temperature phase diagram of water

Figure 5.1. Average temperature difference $\Delta T$ between the evaporator and condenser sections of an FP-OHP

Figure 5.2. Neutron radiograph of the evaporator section of a copper FP-OHP partially filled with water 
Figure 5.3. (left) Plan view of the MURR core (as of 1983) containing eight ${ }^{235} \mathrm{U}$ fuel elements in a cylindrical arrangement, surrounded by neutron-reflector material consisting of beryllium and graphite wedges. (right) Neutron brightness vs. wavelength at E-port before and after removing filters currently in place

Figure 5.4. Plan view of the MURR beam-port floor (BPF) shown with the current suite of instruments.

Figure 5.5. (a) Schematic of a pinhole-camera geometry used to determine the optimal diameter $(D)$ and location $(L)$ of the aperture 123

Figure 5.6. Diameter of the umbra of the neutron beam at the detector position vs. aperture-detector distance $L$ for selected $L / D$ settings

Figure 5.7. Block diagram of the imaging instrument (NIS) layout (a) upstream and (b) downstream of the flight tube

Figure 5.8. Detailed layout of the existing instrument suite at MURR including the proposed NIS at E-port

Figure 5.9. Total microscopic cross-sections of naturally occurring cadmium, ${ }^{56} \mathrm{Fe},{ }^{10} \mathrm{~B}$, ${ }^{1} \mathrm{H}$, and ${ }^{208} \mathrm{~Pb}$ 133

Figure 5.10. 3D sketch of the NIS conceptual design at E-port at MURR 140

Figure 5.11. Top view of the NIS 141

Figure 5.12. Side view of the NIS. 142

Figure 5.13. Trimetric view of the NIS 143

Figure 5.14. Total (calculated) neutron cross section of sapphire at temperatures of $296 \mathrm{~K}$ and $80 \mathrm{~K}$ vs. neutron energy . 145

Figure 5.15. Experimental data for the total neutron cross section of bismuth at temperature of $100 \mathrm{~K}$ vs. neutron energy. 145

Figure 5.16. Semi-log plot of the attenuation coefficient $\Sigma$ of Bi at $100 \mathrm{~K}$ vs. neutron wavelength 146

Figure 5.17. Simulation results of the NIS for the $L / D=400$ setting 147

Figure A.1. Temperature-dependent elastic neutron scattering scans of a CuO-coated sample with $120 \mu \mathrm{l}$ of water added

Figure A.2. 3D schematic of the main body of the sample cell used for neutron scattering experiments 155

Figure B.1. Elastic scattering intensity summed over all 16 detectors vs. temperature as measured on the HFBS for a sample of 10 monolayers of $\mathrm{C}_{32}$ alkane material adsorbed onto $100 \mathrm{Si}$ wafers 158 
Figure B.2. Intensity of elastically scattered neutrons vs. temperature, summed over all 16

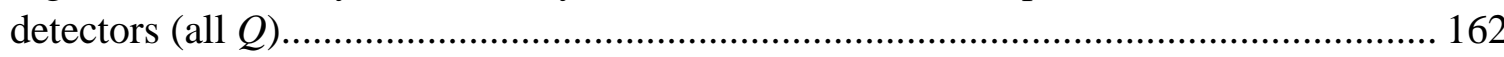

Figure C.1. Diffraction patterns of the CuO-coated sample hydrated with $120 \mu \mathrm{l}$ of $\mathrm{D}_{2} \mathrm{O}$

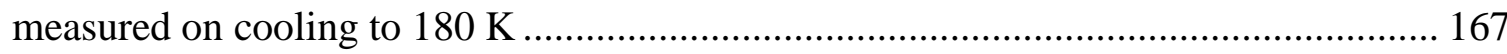

Figure C.2. Diffraction scans of the CuO-coated sample hydrated with $240 \mu \mathrm{l}$ of $\mathrm{D}_{2} \mathrm{O}$

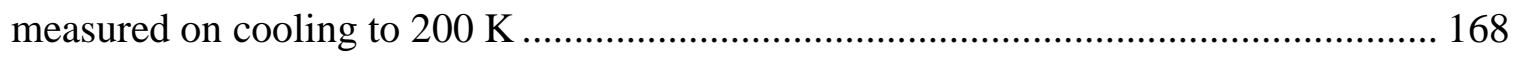

Figure C.3. Example of a Gaussian line shape fit (Eq. (C.1)) to the (100) Bragg peak of $\mathrm{D}_{2} \mathrm{O}$ hexagonal ice

Figure C.4. Background levels of several diffraction scans and beam-open background

Figure C.5. $\mathrm{Cu}(111)$ Bragg peak position vs. temperature that illustrates an instrumental shift around $260 \mathrm{~K}$ 170

Figure C.6. Observed (black points) and corrected (red points) positions of the $\mathrm{D}_{2} \mathrm{O}(100)$ peak as a function of temperature

Figure C.7. $\mathrm{CuO}(111)$ Bragg peak position vs. temperature that illustrates a shift around $260 \mathrm{~K}$ 173

Figure C.8. Observed (black points) and corrected (red points) positions of the $\mathrm{D}_{2} \mathrm{O}(100)$ peak vs. temperature 


\section{Abstract}

Recently, it has been shown that superhydrophilic coatings of "grass-like" cupric oxide $(\mathrm{CuO})$ nanostructures can significantly improve the thermal performance of heat transfer devices known as oscillating heat pipes (OHPs). The origin of this enhanced performance is currently unknown, but it is believed to be attributed to the thin film of interfacial water supported by the nanostructures that coat the OHP's interior surface. The aim of this work is to investigate the microscopic origin of enhanced heat transfer at the $\mathrm{CuO}$ surface by studying the structure, morphology, freezing/melting behavior, and dynamics of the water in proximity to the $\mathrm{CuO}$ coating over time and length scales that span picoseconds-to-seconds and angstroms-to-millimeters, respectively.

Water contact angle measurements show that the native (bare) copper surface of the OHP is weakly hydrophilic, whereas water readily wets to the nanostructured $\mathrm{CuO}$ coating with a contact angle of $\sim 0^{\circ}$. In situ imaging of water condensation in a scanning electron microscope shows that micron-sized droplets form a web-like morphology within the $\mathrm{CuO}$ nanostructures, filling the funnel-shaped pores from base-to-peak. Further evidence of a strong interaction of water with the $\mathrm{CuO}$ nanostructures comes from our high-energy-resolution elastic neutron scattering measurements, which show a continuous freezing transition of the water near the nanostructures over a temperature range of 200$280 \mathrm{~K}$. The temperature dependence of the elastic intensity points to two types of water with different freezing behavior and which are located at different distances from the $\mathrm{CuO}$ nanostructures. The gradual increase in the elastic intensity on cooling suggests the water freezes into an amorphous solid below $260 \mathrm{~K}$. 
Neutron diffraction scans of $\mathrm{D}_{2} \mathrm{O}$-hydrated $\mathrm{CuO}$ samples show no Bragg peaks indicative of the growth of crystalline ice. At higher hydration levels, we observed hexagonal ice form around $260 \mathrm{~K}$. These Bragg peaks were broader than those of water near bare $\mathrm{Cu}$ surfaces, indicating the growth of crystals of a smaller size $(\sim 30 \mathrm{~nm})$ on the $\mathrm{CuO}$ coating as the temperature is lowered to $230 \mathrm{~K}$. Vibrational spectra of the water in the $\mathrm{CuO}$ samples at a temperature of $5 \mathrm{~K}$ measured by inelastic neutron scattering provide supporting evidence that interfacial water solidifies into amorphous ice as evidenced by changes in the H-bonding between water molecules.

From quasielastic neutron scattering, we find evidence of two populations of water that diffuse on three different time scales in the temperature range 230-270 K: 1) pop. 1 strongly interacts with the $\mathrm{CuO}$ surfaces and performs slow, bounded translational motion; 2) pop. 2 is located further from the $\mathrm{CuO}$ surface and performs faster translational motion at a rate similar to that of bulk supercooled water at $\sim 250 \mathrm{~K}$; and, 3) below $240 \mathrm{~K}$, the translational motion in pop. 2 freezes out and reveals a fast, rotational motion in pop. 1 near the $\mathrm{CuO}$ surfaces. The temperature and $Q$ dependence of these motions suggests that some of the interfacial water undergoes a liquid-liquid transition between 240 and $230 \mathrm{~K}$.

Our results demonstrate that water near superhydrophilic $\mathrm{CuO}$ nanostructures exhibits low-temperature anomalies in its structure and dynamics at the molecular level-a direction of research that has both applied and fundamental interest. The significantly altered structure and dynamics of the interfacial water could affect the boundary conditions for bulk water motion inside of an OHP during its operation. To test this hypothesis, we have proposed time-resolved neutron imaging experiments to characterize the kinetics of water oscillations in CuO-coated OHPs. 


\section{Chapter 1}

\section{Introduction to Oscillating Heat Pipes and}

\section{Hydrophilic Materials}

In this chapter, we discuss the motivation for, and development of, heat transfer devices known as heat pipes, a key technology used in the electronics-cooling industry. In Sec. 1.2, we describe the oscillating heat pipe (OHP) as well as the hydrophilic, nanostructured coatings used to enhance OHP performance compared to untreated OHPs. In consideration of the numerous reports of enhanced heat transfer by hydrophilic coatings, in Sec. 1.3 we review recent literature pertaining to hydrophilic and so-called superhydrophilic materials to help explain the microscopic mechanism of enhanced heat transfer. We find that research on superhydrophilic surfaces has centered around their fabrication, applications, and their macroscopic characterization (e.g., by water contact angle measurements). In the experiments presented in this work, our aim is to explore the wetting behavior, structure, and dynamics of water near a hydrophilic, nanostructured cupric oxide $(\mathrm{CuO})$ surface compared to bulk water at the microscopic level. These studies provide a previously underutilized methodology - neutron scattering - for investigating superhydrophilicity at the fundamental level, which can be used for rational OHP design and fabrication. 


\subsection{Motivation}

Microelectronics and computer technologies have advanced significantly over the last 50 years in accordance with Moore's law - a projection based on historical trends that the number of transistors per unit area of an integrated circuit will roughly double every two years [1-2]. The increasing miniaturization, complexity, and power consumption of each new generation of devices has led to an increase in the heat generated in power-dense components (e.g., the CPU), which can negatively affect their performance, reliability, and lifetime. For many years, heat sinks composed of thermally conductive material like aluminum, stainless steel, or copper have been used to facilitate the removal of heat via conduction and radiation to the ambient. However, local heat

fluxes in the range $10^{2}-10^{3} \mathrm{~W} \cdot \mathrm{cm}^{-2} \cdot{ }^{\circ} \mathrm{C}^{-1}$, generated by next-generation electronics [3], are anticipated to exceed the cooling capacity of traditional heat-removal devices [3-4]. The heat dissipation problem was highlighted by G. Eastman in his 1968 article The Heat Pipe [5], where he noted that a heat input of 10,000 watts at one end of a solid copper rod (1-inch diameter and 1-foot long) would result in a temperature difference between the ends of the rod exceeding 30,000 degrees Fahrenheit - about three times hotter than the surface of the sun! Therefore, the development of heat transfer devices is a critical area of research needed to address growing concerns for the deleterious effects of waste heat. 


\subsection{Development of Oscillating Heat Pipes (OHPs)}

The "heat pipe" emerged in the 1960s at Los Alamos Scientific Laboratory and was designed to manage heat generated in spacecraft electronics [5]. It was quickly realized that the heat pipe's ability to transfer large quantities of heat over long distances without loss could be extended to other applications like power generation, solar heating, water desalination, HVAC systems, food processing, and microelectronics cooling [6-8]. Even today, initiatives like nuclear thermal propulsion and power generation identify heat pipes as key to future space exploration [9-10]. Heat pipes are passive devices in that no external power is required to sustain their operation, aside from the heat energy provided by the heat source. Construction of this "conventional" heat pipe (CHP) is illustrated in Fig. 1.1, which resembles that of a gravity assisted heat exchanger (thermosyphon) with notable differences, namely its orientation and use of a wick structure.

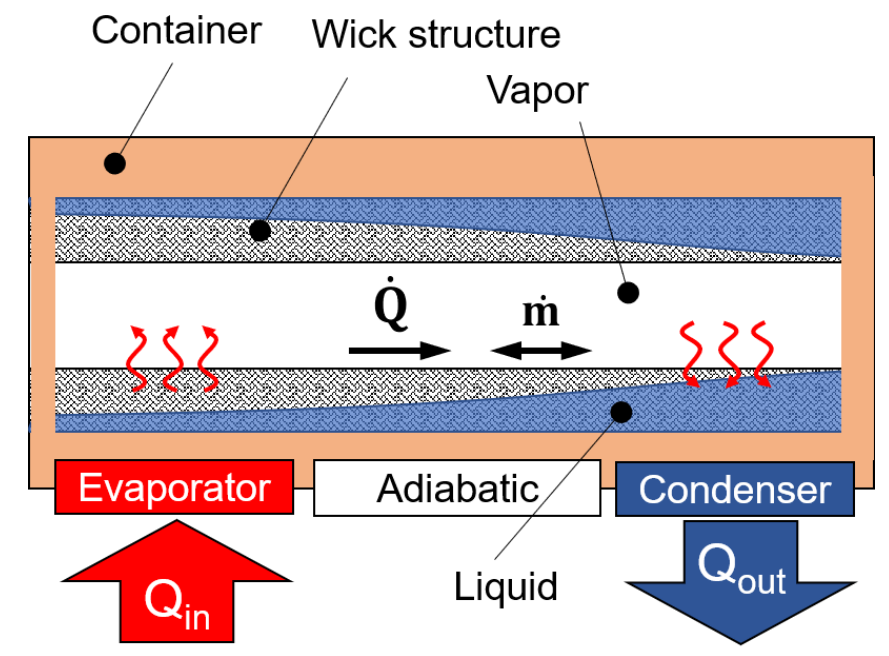

Figure 1.1. Illustration of a conventional heat pipe (CHP) consisting of a hollow container whose interior is lined with a wick structure and partially filled with a working fluid. Heat $\left(Q_{\text {in }}\right)$ introduced at one end (evaporator) is absorbed by the working fluid in the form of latent and sensible heat. Vapor (i்) and heat $(\dot{\mathbf{Q}})$ transport without loss through the adiabatic section to the condenser where the heat $\left(Q_{\text {out }}\right)$ exits to a heat sink. The liquid phase returns to the evaporator section via capillary forces of the wicking structure. Adapted from Ref. [11]. Not drawn to scale. 
The CHP is highly versatile and can be designed in nearly any geometry as it consists of three basic components: a partially evacuated tube, capillary/wick structure, and a volatile working fluid [11]. Taking Fig. 1.1 as an example, the CHP is divided into three regions: evaporator, adiabatic, and condenser sections. By placing the evaporator section in contact with a heat source, heat conducts through the container wall/wick into the working fluid. Once the fluid reaches the temperature corresponding to its saturation vapor pressure, the evaporating liquid creates a pressure difference between the evaporator and condenser sections. This pressure difference drives the transport of vapor through the adiabatic section to the condenser section. By connecting the condenser to a heat sink at a temperature lower than that corresponding to the saturation vapor pressure of the working fluid, vapor condenses back into liquid and thereby releases latent and sensible heat externally. Liquid condensate transports back to the evaporator section primarily by capillary forces of the wick structure as well as by gravity [12]. Sustained vapor transport via evaporation-condensation cycles forms the basis of the heat pipe design and its exceptionally high thermal conductivity.

The materials used to construct the CHP depend on its operating conditions. Choice of the working fluid is based on the operational temperature range as well as the fluid's latent heat of vaporization, surface tension, and viscosity. Water, acetone, and ammonia are typical for low-temperature $\left(-70-270^{\circ} \mathrm{C}\right)$ applications [7]. The container material is almost always metallic and designed for high thermal conductivity, machinability, and chemical compatibility with the working fluid. A typical commercialized heat pipe is constructed from thin copper tubing and charged with water as the working fluid, which is used to cool electronic components like those in laptop computers as shown in Fig. 1.2. 


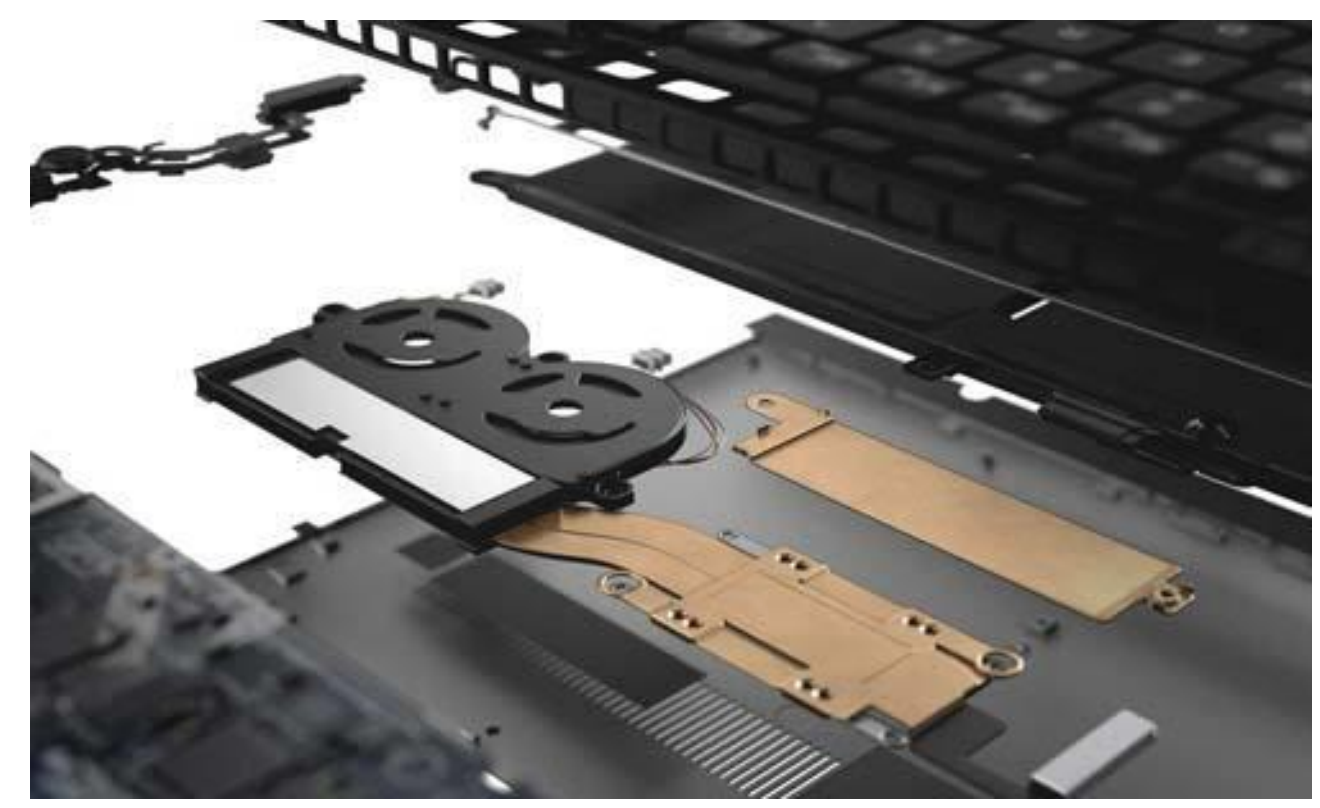

Figure 1.2. Exploded view of a notebook computer with a copper heat pipe integrated in the cooling system (GORE ${ }^{\mathrm{TM}}$ Thermal Insulation, Dell XPS). Source: Dell.com (2019).

In the early 1990s, Akachi patented the oscillating heat pipe (OHP) [13-14], which eliminated the need for a wick structure to return liquid to the evaporator section. This advance was achieved by reducing the inner diameter of the tube below the capillary length of the working fluid $(\sim 2.7 \mathrm{~mm}$ for water [15]) such that the fluid forms a chain of liquid "slugs" and vapor "bubbles" via surface tension (see Fig. 1.3a) [11]. In addition, the tube is bent into a series of parallel turns that wind between the evaporator and condenser sections. Expansion and contraction of the vapor bubbles due to heat addition and rejection, respectively, drives an oscillatory motion of the slugs and bubbles. This added convective component increases the transfer of sensible heat compared to the CHP. The most common OHP designs are tubular (T-OHP) and flat-plate (FP-OHP) where the latter is shown in Fig. 1.3b. Thermal performance is measured by the temperature difference $(\Delta T)$ between the evaporator $\left(T_{\text {evap }}\right)$ and condenser $\left(T_{\text {cond }}\right)$ sections, which can be converted to an effective thermal resistance where a lower resistance (lower $\Delta T$ ) is desired for heat-transfer applications. 

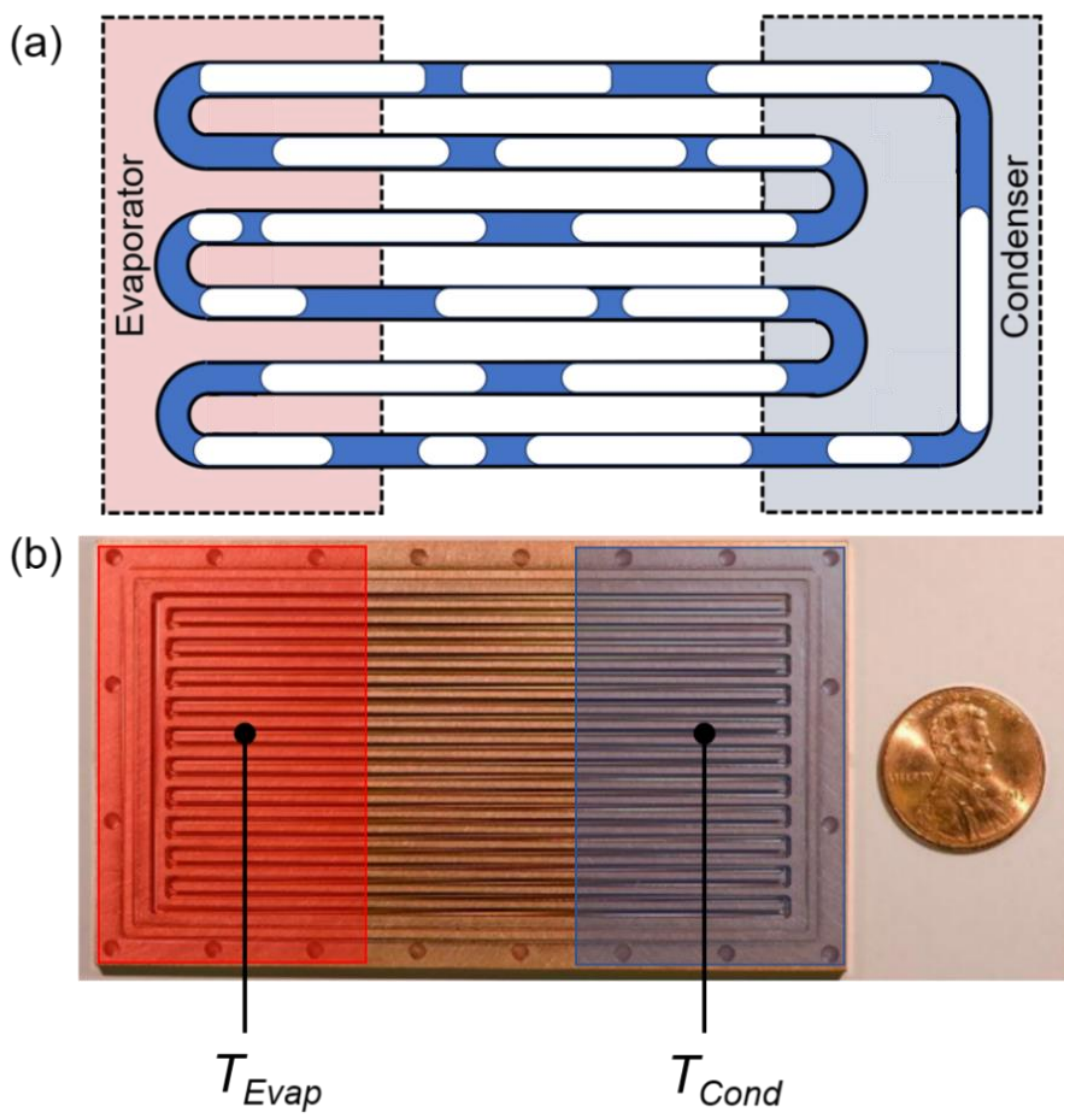

Figure 1.3. (a) Diagram and (b) image of a closed-loop OHP consisting of a narrow capillary tube meandering between a heat source (evaporator) and heat sink (condenser). The interior is partially filled with a working fluid, which separates into liquid "slugs" (blue regions) and vapor "bubbles" (white regions) due to surface tension. (b) An FPOHP with the cover plate removed, revealing the inner channels that were machined into the copper plate. Image reproduced from Ref. [16].

The next evolution of OHP design incorporated nanofluids, which are nanoparticles suspended in the working fluid that lead to an increase in heat capacity and a decrease in OHP thermal resistance. In many cases, the origin of this enhanced performance is related to the deposition of nanoparticles on the OHP's channel surface during the boiling phase, thereby forming a nanostructured coating $[6,17-19]$. While there is clear link between these coatings and improved heat transfer, the definitive microscopic mechanisms are still to be determined. 
In a recent study by Zhang et al. [16], the temperature difference between the evaporator and condenser sections, i.e., the thermal performance, of a water-charged copper FP-OHP (Fig. 1.3b) was enhanced by selectively treating the evaporator or condenser sections with a coating of cupric oxide $(\mathrm{CuO})$ nanostructures. The $\mathrm{CuO}$ coating, which has been reproduced in the scanning electron microscope (SEM) image in Fig. 1.4, was grown by partially immersing the copper OHP into a hot alkaline solution $[\mathbf{1 6 , 2 0}]$. The measured water contact angle of $\sim 12^{\circ}$ revealed that the resulting $\mathrm{CuO}$ coating is strongly hydrophilic, which contrasts with the initially untreated copper with a contact angle of $\sim 60^{\circ}[\mathbf{1 6}]$. To explain the improved heat transfer performance for nanostructures placed in the evaporator section, Zhang et al. proposed that the $\mathrm{CuO}$ coating enhances thin-film evaporation due to the thin layer of water left behind by a moving liquid slug [16].

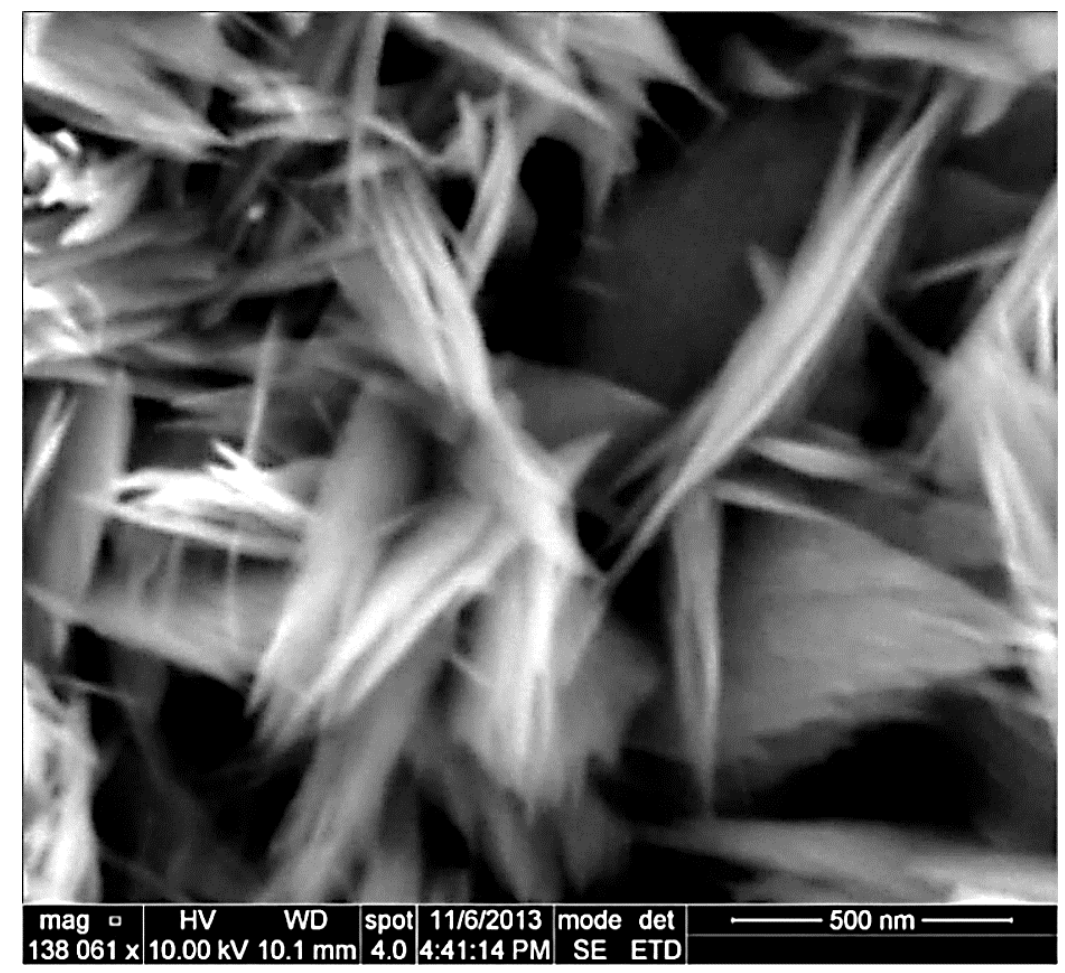

Figure 1.4. SEM image of $\mathrm{CuO}$ nanostructures grown on copper via hot alkaline bath $[\mathbf{1 6}, \mathbf{2 0}]$. Note the triangular "grass-like" morphology. Image reproduced from Ref. [16]. 
The conclusions of Zhang et al. [16] support those reached by others [6,21-23]. For example, the mathematical model developed by Ma et al. [21] identifies thin-film evaporation in the evaporator section as key to designing highly efficient OHPs. Experiments by Ji et al. [22] showed that a hydrophilic copper T-OHP has a lower thermal resistance over a heat input range of 0-200 W compared to a hydrophobic OHP. This performance increase was attributed to the presence of a thin liquid film in the hydrophilic evaporator section that was absent in the hydrophobic case.

In addition to thin-film evaporation, nanostructured coatings have also led to enhanced heat transfer in the boiling regime. Qu et al. [24] investigated the effects of surface roughness on OHP thermal performance by machining helical micro-grooves into copper T-OHP channels. The thermal resistance of the micro-grooved OHP was lower than the smooth OHP, and the grooves helped to delay the creation of a thermally insulating vapor layer during the transition from nucleate to film boiling. The latter also marks the maximum exchangeable heat flux, i.e. the "critical heat flux" (CHF), between the hot container wall and the working fluid [25]. $\mathrm{Xu}$ and $\mathrm{Li}[\mathbf{2 6}]$ determined that a $\mathrm{CuO}-$ nanoflower-coated copper OHP increased the CHF compared to an uncoated OHP with $\mathrm{mm}$-sized grooves. Moreover, they observed a factor of two difference in the growth rate of vapor bubbles facilitated by the dense array of nanostructures. These combined effects are particularly useful in applications which rely critically on devices operating below the CHF threshold like, for example, the Fukushima Daiichi boiling-water nuclear reactor [27]. In fact, heat pipe technology is being considered for passive heat removal in the event of power loss during a reactor shutdown (see Fig. 14 and related discussion in Ref. [7]). While the primary use of a wick or capillary structure in a CHP is to move liquid 
from the condenser to the evaporator, its use in OHPs is to promote constant contact between the liquid working fluid and the hot container wall $[\mathbf{1 5 , 2 5 , 2 8}]$.

In addition to enhanced two-phase heat transfer, interfacial water also provides some hydrodynamic benefits to OHP performance. In a series of high-speed photography and thermal performance experiments [23,29-30], Hao et al. observed changes in the motion of water slugs in copper FP-OHPs with different surface treatments. From highspeed photography, the amplitude and velocity of liquid slugs in the superhydrophilic $(\mathrm{CuO})$ and hydrophilic $(\mathrm{CuO}$ removed by acid) OHPs were higher than those of the untreated and hydrophobic ( $n$-octadecyl mercaptan-coated) copper OHPs. They concluded from these tests that it was a thin film of water that lubricates vapor bubbles as they pass over the nanostructures, thereby reducing the overall flow resistance. This in turn led to an improvement in sensible heat transfer caused by larger amplitude and higher velocity of liquid slugs compared to untreated and hydrophobic OHPs. In a related set of experiments, Cubaud et al. [31] studied two-phase flow characteristics of water bubbles moving in microchannels with square cross-sections. They also observed that hydrophilic surface treatments created a thin film of water, which can support wedge or slug flow (see Fig. 4b and 4c in Ref. [31]) based on the stability of the film.

To summarize, there are several proposed mechanisms that might explain the observed enhancement in heat transfer in micro/nanostructured OHPs. One of these depends on a high capillary pressure induced by the nanostructures, which pumps liquid to recently dried regions such as in boiling heat transfer $[\mathbf{6 , 3 2}]$. Accordingly, this effect is bounded by the capillary limit. Similarly, superhydrophilic coatings promote water's physical and/or chemical bonding to the OHP surface, which enhances liquid spreading 
and thin-film evaporation. Superior liquid spreading of liquid on $\mathrm{CuO}$ compared to uncoated copper leads to an evenly distributed water layer over the OHP surface and minimizes the formation of temperature hot spots [6]. From another perspective, the presence of a thin liquid film at the container wall lowers the flow resistance of the oscillating liquid slugs and therefore leads to an enhancement in sensible heat transfer $[6,23]$.

\subsection{Surface Wetting, Superhydrophilicity, and the}

\section{Unusual Properties of Interfacial and Pore Water}

The previous section highlights the need to understand the extent to which the thin film of water within nanostructured coatings affects the transport of heat and mass around it. To address this question, we first turn to the study of surface wetting and wettability, which dates back to the work by Thomas Young over 200 years ago and his concept of the contact angle [33-34]. Over a century later, in the 1930s and 1940s, theoretical models were developed, namely by Wenzel [35] and Cassie and Baxter [36], to account for the observed effects of surface roughness on wettability. Despite challenges to contact angle analyses, especially in the context of superwetting (see, e.g., Refs. [34,37-38]), they are still used to this day as convenient models to describe wetting behavior.

Materials that demonstrate extreme wetting behavior like superhydrophilicity and superhydrophobocity are typically characterized by extreme values in their contact angle measurements. These wetting behaviors have drawn considerable attention particularly because of their wide range of practical applications [34,39-42]. For example, the 
spontaneous wetting facilitated by superhydrophilic materials lends itself useful in selfcleaning $[\mathbf{4 0}, \mathbf{4 3}]$, anti-fogging/icing $[\mathbf{3 9}, \mathbf{4 3}]$, biomedical (antibacterial and low-drag) $[39,41,43]$, thin-film patterning [44], heat-transfer $[\mathbf{1 2 , 1 5 - 1 6 , 2 0 - 2 1}]$, and water/oil filtration $[39,45]$ applications. The ability to form uniformly thick films of water can have a dramatic effect on reducing sample preparation time for cryo-electron microscopy. Here, sample stability and electron transparency are strongly dependent on the thickness of the vitreous ice film [46], and superhydrophilic coatings like $\mathrm{CuO}$ offer a possibility to control film thickness [47].

Materials designed for superwetting are typically modeled from nature, i.e., "bioinspired." For example, the water repellency of the lotus leaf has become iconic for demonstrating superhydrophobicity where rainwater readily curls-up into spherical droplets that roll off the leaf's surface, carrying dust or other contamination. Artificial coatings that imitate this behavior like car wax have immediate application to glass surfaces to reduce water and ice adhesion. At the other end of extreme wetting, some fish (e.g., N. septentrionalis) can survive in oil contaminated seas because of their superhydrophilic and oil repellent "oeleophobic" scales [48-49]. Fabrication of these bioinspired materials has thus become active area in materials research and surface engineering where characterization of the water-solid interface is of fundamental importance.

\subsubsection{Contact Angle}

Generally speaking, the difference between hydrophilicity and hydrophobicity is qualitative in that hydrophilic surfaces are those which have a high affinity for water whereas hydrophobic surfaces are water-repelling. The level of affinity towards water is 
based on a competition between adhesive (water-solid) and cohesive (water-water) forces, which may include electrostatic, dispersion, induction, repulsion, and hydrogen bonding $[\mathbf{5 0 , 5 1}]$. With this understanding, modifications of surface hydrophilicity are made by changes to the surface chemistry and roughness. Generally, surfaces that are intrinsically hydrophilic or hydrophobic are made more so by increasing surface roughness, which might or might not affect the value of the water contact angle (CA) $\theta$, as illustrated in Fig. 1.5.
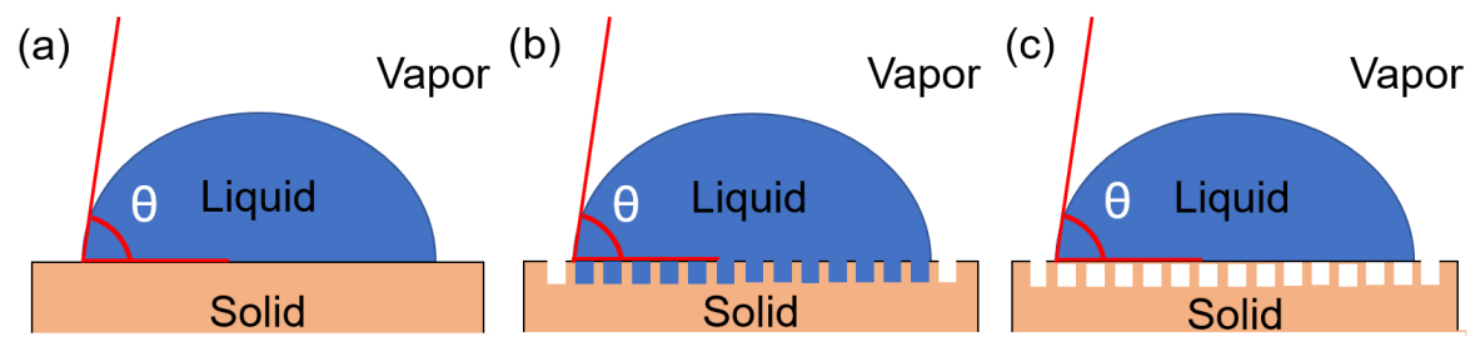

Figure 1.5. Schematic diagram of a water contact angle $\theta$ measured at the contact line formed at the intersection of three phases: solid substrate, liquid water droplet, and surrounding vapor. Identical contact angles are shown for the (a) Young [33-34], (b) Wenzel [35], and (c) Cassie-Baxter models [36] (see text).

Typically, an equilibrium CA represents the balance of forces at the three-phase (solid, liquid, vapor) contact line for a perfectly flat, chemically homogeneous solid surface. This balance can be described using Young's equation [33-34],

$$
\gamma_{s v}-\gamma_{s l}=\gamma_{l v} \cos \theta
$$

where $\gamma$ is surface tension or surface free energy $([\mathrm{N} / \mathrm{m}])$ and the subscripts $s, l$, and $v$ denote the solid, liquid, and vapor phases, respectively. Wenzel [35] proposed that surface roughness (see Fig. 1.5b) enhances wettability through a roughness factor $r$ (ratio of true-to-projected surface area), which modifies Young's equation (Eq. (1.1)),

$$
\cos \theta=\frac{\cos \theta_{a}}{r}
$$


where $\theta_{a}$ is the experimentally accessible apparent-CA. A similar empirical expression was derived by Cassie and Baxter [36] to account for the rough composite surface (solid and vapor) below the water droplet as shown in Fig. 1.5c.

Although observations generally obey Eq. (1.2), it is difficult, if not impossible, to extract $\theta_{a}$ from systems that display ideal wetting $\left(\theta_{a} \rightarrow 0\right)$. Moreover, physical and chemical heterogeneities can pin the contact line, which skew the measurement of $\theta_{a}$, i.e., the angle is not unique $[\mathbf{4 2 , 5 2 - 5 3}]$. These factors imply that there are multiple equilibrium wetting states, which can be explored by advancing and receding water droplets through dynamic-CA measurements. Nevertheless, it is convenient to label materials that display static CAs of $<10^{\circ}$ as superhydrophilic and $\theta_{a}>150^{\circ}$ as superhydrophobic $[34,39,49]$.

The superwetting states discussed above can only be accessed by creating a sufficiently rough interface of an already hydrophilic or hydrophobic material. To demonstrate, Nishino et al. chemically modified a flat glass surface with a coating of $n$ Perfluroeicosane deposited on a hexagonal close-packed lattice with the intent of making the glass superhydrophobic [54]. They found that the average of the advancing and receding CAs was $119^{\circ}$, which is low compared to the static $\sim 160^{\circ} \mathrm{CA}$ for the iconic lotus leaf [55]. Because the surface free energy of the coating $\left(6.7 \mathrm{~mJ} / \mathrm{m}^{2}\right)$ is considered one of the lowest of any solid, this result shows that microscopic structures, like the waxy papillae of the lotus leaf, are key to creating a superhydrophobic surface. In fact, one can take an initially superhydrophilic, nanostructured surface like $\mathrm{CuO}[\mathbf{5 6}]$ and effectively lower its surface free energy with a fluorinated or polymer coating to achieve superhydrophobicity $[\mathbf{1 5 , 5 7 ]}$. Superhydrophilicity is in some ways easier to achieve 
because a hydrophilic material can be artificially roughened. In case of metals, they can be chemically treated to promote the growth of a rough oxide layer [15]. Alternatively, some oxides like $\mathrm{ZnO}$ or $\mathrm{TiO}_{2}$ can be exposed to $\mathrm{UV}$ radiation to create oxygen vacancies at the surface that alter the chemical composition of the surface to facilitate dissociative water adsorption [58]. The net effect is a short-lived reduction of the static water CA to nearly zero [40]. The recent review article by Liu et al. [44] provides more detail on the design criteria of superwetting surfaces.

\subsubsection{Review of Interfacial and Confined Water}

In comparison to the abundant research devoted to the fabrication and application of superwetting materials [e.g., 39-40,42,59-60], less attention has been paid to understand physical and chemical interactions that take place at the microscopic interface between the oxide surface and water. Indeed, confined and interfacial water are ubiquitous in nature and found in places like cracks in rocks, soil, interior of cells [61-63] or even among the cosmos on interstellar dust [64]. In many cases, interfacial water is not a static bystander but rather an active participant in surface interactions like protein and membrane functions [65]. In fact, most of the water in our bodies is not in its bulk form but rather it can be considered interfacial [66]. Figure 1.6 illustrates the distinction between interfacial and bulk water on flat surfaces and within pores.

As will be discussed below, the properties of interfacial and confined water often differ from those of the bulk and include phenomena like polymorphism, suppressed or altered phase transition(s), density changes, hysteresis, layering effects, and enhanced or suppressed mobility. Given the ubiquity of interfacial water and its apparent systemdependent properties, there are fundamental questions that need to be addressed, 
including its thickness, viscosity, reactivity and bonding to the substrate, thermodynamic properties, orientation, structure, and mobility compared to bulk water.
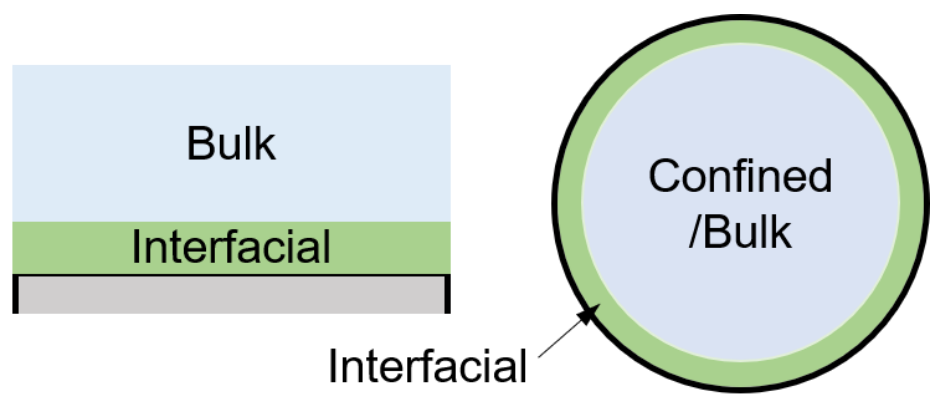

Figure 1.6. Generalized continuum models of interfacial and bulk water, illustrated for flat (left) and porous (right) materials.

The number of journal articles and books dedicated to the understanding of water at various interfaces is extremely vast; so, for practical purposes, the scope of this section is limited to a review of the freezing/melting behavior, structure, and dynamics of water in hydrophilic porous materials with a focus on experimental results. In particular, we are interested in the thin layer of water that resides at the surface of these porous materials. Recent development of prototypical porous materials that mimic aspects of natural environments has enabled detailed experimental studies of interfacial water. Some of these materials include templated-silica (MCM- $n$, SBA- $n$ ), zeolites, microemulsions (aerosol-OT), carbon nanotubes and aerogels, graphite oxide, mineral clays, and other porous glasses (Vycor, silica gel) [63,67]. MCM-41 and SBA-15 are among the most studied systems $[\mathbf{6 3 , 6 8 - 7 0}]$ as they can be prepared and purified relatively easily, have extremely high specific surface areas $\left(10^{2}-10^{3} \mathrm{~m}^{2} / \mathrm{g}\right)$, and can be chemically modified to impart varying degrees of hydrophilicity. Furthermore, their programmable pore shapes and sizes reduce the ambiguity of experimental results. Moreover, the characteristic length and time scales of water structure and dynamics are amenable to studies via 
computer simulations to test the effects of pore size and hydrophilicity [70-72]. Thus, throughout this thesis we frequently compare our findings from neutron scattering and diffraction studies to those of silicas like MCM and SBA.

Perhaps one of the most documented results from studies of water confined in porous materials, especially mesoporous silicas, has been the effect of pore size on water's equilibrium melting temperature $T_{m}$. Studies on the freezing and melting of water in silica pores have shown a systematic depression of $T_{m}$ with decreasing pore diameter to temperatures well below $273 \mathrm{~K}$, as characterized by DSC (differential scanning calorimetry), NMR (nuclear magnetic resonance), Raman and IR spectroscopy, and neutron and $\mathrm{x}$-ray diffraction $[\mathbf{6 3 , 7 0 - 7 1 , 7 3 ]}$. For pore diameters $\leq 21 \AA$, crystallization can be avoided altogether $[\mathbf{6 6}, \mathbf{7 4}]$; and, the hysteresis between freezing and melting vanishes for many pores with sizes less than $\sim 27 \AA$, which implies an end to the firstorder transition character of bulk water $[\mathbf{7 1 , 7 5}]$. Similar occurrences of nonfreezing pore and interfacial water come from water intercalated in graphite oxide [76], minerals [77], and hydrogels [78].

In some cases, confinement of water to narrow pores or to hydrophilic surfaces is considered to be a "trick" to study the physics of supercooled water in the temperature range $\sim 150-235 \mathrm{~K}$, which for many years has been the experimentally inaccessible "noman's land" of water's $P$ - $T$ phase diagram [70,79-81]. Access to this region would be interesting because, based purely on theoretical predictions and computer simulations [82-83], there exists a phase boundary that extends from the liquid-gas coexistence line into the single-phase region; further, it separates high- and low-density phases of supercooled water at temperatures above and below the transition, respectively, and could 
explain many of the anomalies of supercooled water. This proposed liquid-liquid transition alludes to the experimentally observed first-order transition between highdensity (HDA) and low-density (LDA) amorphous ice [79]. In other words, LDA and HDA are thought to be the vitrified forms of low- and high-density liquid water, respectively [84]. At ambient pressure, the transition temperature $T_{c}$ is in the proximity of $228 \mathrm{~K}$ where many physical quantities tend to diverge $[\mathbf{8 2 , 8 4}]$. Crossing this so-called "Widom Line" from above $T_{c}$ could be associated with a change in the diffusion coefficient $D(T)$ from Arrhenius ("fragile") to non-Arrhenius ("strong") temperature dependence [82]. These theoretical studies were substantiated by the experimental work of Liu et al. [85] who observed a dynamic crossover in the average relaxation time of water confined in $14 \AA$ A-dia. pores of MCM-48-S at $224 \mathrm{~K}$ at ambient pressure. On this basis, there are now many more examples of dynamic crossovers of water in both hard and soft confinement [63]. It is still unclear, however, whether confined water can be considered as a viable model of bulk supercooled water or its relation to a true liquidliquid critical point $[63,87-89]$. Nevertheless, it is apparent that confined and interfacial water pose significant challenges to our understanding of its behavior, especially at temperatures below is bulk melting point.

Molecular dynamics simulations have confirmed the presence of a liquid-like layer of water that wets silica pore walls (pore diameter of $30 \AA$ ) where a large fraction of it does not participate in crystallization [71]. In addition, Moore et al. [70] determined that, below the bulk freezing point, the fraction of liquid-like water in $40 \AA$ diameter silica pores is systematically higher in hydrophilic pores than in hydrophobic pores where the distinction between the two wetting states is based on the surface interaction potential 
$\varepsilon_{w p}(>0.3 \mathrm{kcal} / \mathrm{mol}$ corresponds to hydrophilic). In cooling the water well below the melting point (e.g., $190 \mathrm{~K}$ ), the freezing behavior indicates that a significant fraction (up to $50 \%$ ) of interfacial water will not crystallize prior to vitrification. They concluded this study by pointing out that the melting temperatures of bulk-like water in the pores are not related to the contact angles or adsorption energies of water molecules in a trivial way.

The melting point depression of water in nanosized pores can be modeled using a modified form of the Gibbs-Thomson (GT) equation [70-71,87,90-91],

$$
T_{m}=T_{0}-\frac{C}{R-t}
$$

where $T_{0}$ is the melting point for bulk water $(273 \mathrm{~K}), R$ is the pore radius, $C$ is a constant $[\mathrm{K} \cdot \mathrm{nm}]$ that depends on the free energy of the system, and $t$ is a fitting parameter that is often interpreted as the thickness of a nonfreezing layer of water. The value of $t$ varies between different systems but is typically on the order of $0.3-1.0 \mathrm{~nm}$, or 1-3 equivalent monolayers of water $[\mathbf{7 0 , 7 3 , 7 5}]$. By this model, confinement effectively accounts for a change in the Gibbs free energy relative to bulk water [68].

The GT equation has proven useful in determining the melting point of confined water in many systems, but it provides no insight regarding the structure of the nonfreezing water layer or its dependence on surface wettability $[\mathbf{6 6 , 6 9 , 7 1 ]}$. Using X-ray and neutron scattering, Jelassi et al. [69] showed that there are distinct differences in the structure, particularly the second nearest neighbors, of water confined in either hydrophilic (unmodified) or hydrophobic (methylated) silica pores having diameters of 50-70 A. They suggested that three of the four H-bonds of a water molecule near a hydrophilic (hydrophobic) surface will form with surface-hydroxyl groups (other water) 
and point towards (away from) the pore wall, altering the density and orientation of the molecules. From the IR measurements by Asay et al. [73], water in the temperature range $10-35{ }^{\circ} \mathrm{C}$ tends to adsorb onto hydroxylated (hydrophilic, $\mathrm{CA}<5^{\circ}$ ) silica surfaces with predominantly solid-like structure (spectroscopically). Furthermore, by increasing the hydration level, the population of solid-like water near the hydrophilic surface increases at a faster rate than the liquid-like water up to $70 \%$ relative-humidity. In contrast, the amount of adsorbed water on modified (slightly hydrophobic, $\mathrm{CA}=45-90^{\circ}$ ) silica is about a third less and both fractions of solid-like and liquid-like water grow at the same rate with increased hydration level. In a review by Bertrand et al. [92], a number of different experimental studies such as small-angle x-ray and neutron scattering, diffraction, and Raman and IR spectroscopy show a gradual decrease in the density of water in porous MCM-41 on cooling from the maximum density around $240-250 \mathrm{~K}$ to a minimum around $180-200 \mathrm{~K}$ at 1 bar of pressure. The derivative of the density with respect to temperature is proportional to the thermal expansivity, which shows a minimum around $240 \mathrm{~K}$ for $\mathrm{D}_{2} \mathrm{O}$ that is strongly confined in MCM-41 (see Fig. 14 in Ref. [92]).

There are a variety of experimental methods used to probe the dynamics related to the freezing and melting of water confined in nano/mesoporous silica. NMR studies by Miyatou et al. [81] revealed that the correlation time of the isotropic rotation of $\mathrm{D}_{2} \mathrm{O}$ molecules, from room-temperature to $\sim 238 \mathrm{~K}$, followed a Vogel-Fulcher-Tammann (VFT) relation. As the temperature approached the freezing point, $\mathrm{D}_{2} \mathrm{O}$ molecules clustered and formed H-bonds before creating a low-density, non-crystalline structure that strongly affected the rotation of water in the spherical SBA-16 micropores. [93-94]. Similarly, temperature dependent NMR studies [68] of $T_{2}$ relaxation of water in SBA-15 
indicate that the disordered (liquid-like) interfacial water as measured by neutron diffraction displays relaxation rate that is intermediate between bulk ice and liquid water. On heating to about $200-220 \mathrm{~K}$, a sharp rise in the $T_{2}$ relaxation was observed and attributed to the rapid onset of rotational diffusion where the water entered a plastic ice state. Then, just prior to the melting event, translational motion set in around $260 \mathrm{~K}$ [68]. This behavior appears to be reversible in cooling and heating scans.

Quasielastic (QENS) and inelastic neutron scattering provide complementary information regarding the anomalous melting and freezing of water confined in porous materials like silica. This is due to the exceptionally high incoherent scattering cross section of hydrogen ( 80.26 barn) compared to the other components: silicon (0.004 barn) and oxygen (0.0008 barn) [95]. Takahara et al. [96] were among the first to study water dynamics in MCM-41 pores using QENS and found that the relaxation time of water in the center of the pore was longer than that of bulk water; but, rotational relaxation rate of the sample with a monolayer of water was nearly the same as that of the interfacial water in the filled pores. They also determined that the diffusion of water was systematically slower in narrower pores where there is a higher fraction of interfacial water. Zanotti et al. [80] reported evidence from QENS measurements that interfacial water $(\sim 1$ monolayer) in Vycor experiences a first-order transition from a low-density to a higher density liquid at $240 \mathrm{~K}$ on heating where rotational and long-range translational motions set in separately around $150 \mathrm{~K}$ and $240 \mathrm{~K}$, respectively. Their neutron diffraction measurements confirmed that, down to $77 \mathrm{~K}$, no Bragg peaks characteristic of crystalline ice were observed at this low hydration level and therefore the water was in an amorphous solid state. 
Kiwilsza et al. [97] used both QENS and NMR to evaluate the dynamics of water in $\sim 55 \AA$ pores of SBA-15 at low hydration level (15\% pore volume). The gradual loss of the free induction decay signal (from NMR) on cooling from $240 \mathrm{~K}$ to $120 \mathrm{~K}$ is interpreted as both a gradual freezing transition and slowing of the mobility of water in the pores as the temperature was reduced. Over this temperature range, the spin-spin relaxation time $T_{2}^{*}$ shows a dynamic transition around $\sim 165 \pm 5 \mathrm{~K}$. From QENS, the temperature-dependent scan of the intensity of elastically scattered neutrons $(\sim 0.9 \mu \mathrm{eV}$ resolution) indicates a gradual melting of the interfacial water with an inflection point around $170 \mathrm{~K}$ that separates the two linear regions. At $210 \mathrm{~K}$, the quasielastic peak can be described by a single Lorentzian function whereas at $250 \mathrm{~K}$ and $300 \mathrm{~K}$ two Lorentzians are required. Each Lorentzian produced an average correlation time that differed significantly from the other. Furthermore, the activation energies of the two different types of motions (short-range vs. long-range hopping) were in good agreement with the values derived by NMR, showing that both techniques were sensitive to the same types of motion.

In summary, it is clear that the behavior of interfacial and confined water is remarkably different than bulk water in many ways. The confinement of water to narrow pores of mesoporous silica has a dramatic effect on the water's ability to freeze into crystalline ice. Furthermore, the differences in surface hydrophilicity are reflected in the different ways that water arranges on the silica wall as well as its mobility compared to water further from the surface. The possibility of a second critical point of water has recently opened an active and exciting area of research that extends to the physical and applied sciences. 


\section{Chapter 2}

\section{The Structure, Morphology, and Wetting}

\section{Properties of Nanostructured $\mathrm{CuO}$}

In this chapter, we describe how we used electron and atomic force microscopy (AFM), water contact-angle, and vapor sorption measurements to characterize $\mathrm{CuO}$ coatings that mimic the interior surfaces of copper flat-plate oscillating heat pipes (FP-OHPs). The nanostructures that were prepared using the oxidation recipe by Liu et al. [98] have been shown to enhance the thermal performance of water-charged FP-OHPs [16]. However, we found that the uniformity of these $\mathrm{CuO}$ coatings was inadequate for the many foils required for our neutron scattering experiments. Therefore, we used another recipe by Nam and Ju [56], which our scanning electron microscope (SEM) images show the oxide to form a nearly identical "grass-like" $\mathrm{CuO}$ morphology as those by Liu et al. [98].

A detailed understanding of the structure and composition of the solid surface is required to interpret our findings from neutron scattering experiments. Therefore, in Sec. 2.1, we characterize the crystallinity, layering structure, and chemical composition of the $\mathrm{CuO}$ coating using a variety of transmission electron microscopy (TEM) techniques. In Sec. 2.2, we discuss our water contact-angle and condensation experiments where the latter reveal the wetting behavior and superhydrophilicity of the $\mathrm{CuO}$ coating on the micron scale. Lastly, in Sec. 2.3, we characterize the pore size, surface area, and water adsorption of the $\mathrm{CuO}$ coating using vapor sorption measurements and AFM. 


\subsection{Growth of "Grass-like" CuO Nanostructures}

Cupric oxide $(\mathrm{CuO})$ nanostructures have recently attracted attention because of their sizedependent physical and chemical properties such as tunable band gap, photoluminescence, electrical resistivity, photocurrent density, and magnetism [99]. These tailorable properties are desirable for a wide range of applications: gas/bio sensing, solar collection, nano/microfluidics, catalysis, field emission ( $p$-type semiconductor), batteries, heat transfer, and filtration [99-100]. Unlike other $3 \mathrm{~d}$ transition-metal oxides, $\mathrm{CuO}$ forms a monoclinic crystal structure (as opposed to the typical rock-salt structure) whose micron-scale morphology can be controlled by tuning growth conditions like temperature, pressure, and concentration of reactants [99].

The nanostructured-CuO coating applied to copper FP-OHPs was grown using the recipe developed by Liu et al. $[\mathbf{1 6 , 9 8}]$ : the native oxide layer of the copper OHP was removed by immersing the $\mathrm{OHP}$ in $3.0 \mathrm{M} \mathrm{HCl}$ solution for $10 \mathrm{~min}$. After rinsing in ethanol and deionized water, the OHP was then immersed in an aqueous solution consisting of $30 \mathrm{mM}$ of $\mathrm{NaOH}$ for 20 hours at $60{ }^{\circ} \mathrm{C}$. After oxidation, the OHP was removed from heating, rinsed in deionized water, and air-dried. This process resulted in a dark coating of copper oxide as shown for copper foils in Fig. 2.1. On preparing several dozen samples required for our neutron scattering experiments, we found that the uniformity and homogeneity of the $\mathrm{CuO}$ layer was inadequate. Therefore, another synthesis option was considered and is discussed below. 

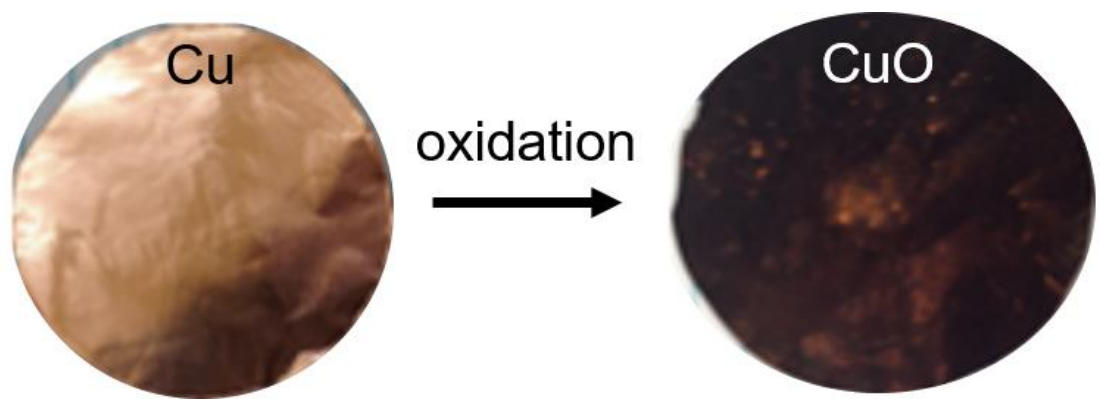

Figure 2.1. Photos of a 5-cm diameter, 12- $\mu \mathrm{m}$ thick copper foil (left) before and (right) after oxidation using the recipe developed by Liu et al. [98].

On inspecting the oxidized foil in Fig. 2.1, we see that the surface is decorated with brown (or greyish) spots. An SEM image of one of these spots is shown in Fig. 2.2 and reveals a different morphology from that of the nanostructures found in the black areas of the foil (Fig. 1.4). A different nanostructure morphology could affect the hydrophilicity of the oxidized foil and therefore the behavior of interfacial water (see, e.g., Fig. A.1). Using energy-dispersive X-ray spectroscopy (EDS), the elemental composition of the discolored areas of the oxidized foils consisted of copper and oxygen, like the $\mathrm{CuO}$ (black) regions; however, the copper signal at $\sim 0.9 \mathrm{keV}$ is markedly enhanced, which indicates the discolored patches are likely composed of the other stable copper oxide phase, $\mathrm{Cu}_{2} \mathrm{O}$, i.e., the oxidation process was incomplete. Attempts were made to achieve higher uniformity of nanostructures by increasing the oxidation time to 28 hours, but the number of samples whose surfaces lacked complete $\mathrm{CuO}$ coverage remained around 10-20\% (out of 100 foils per batch). 


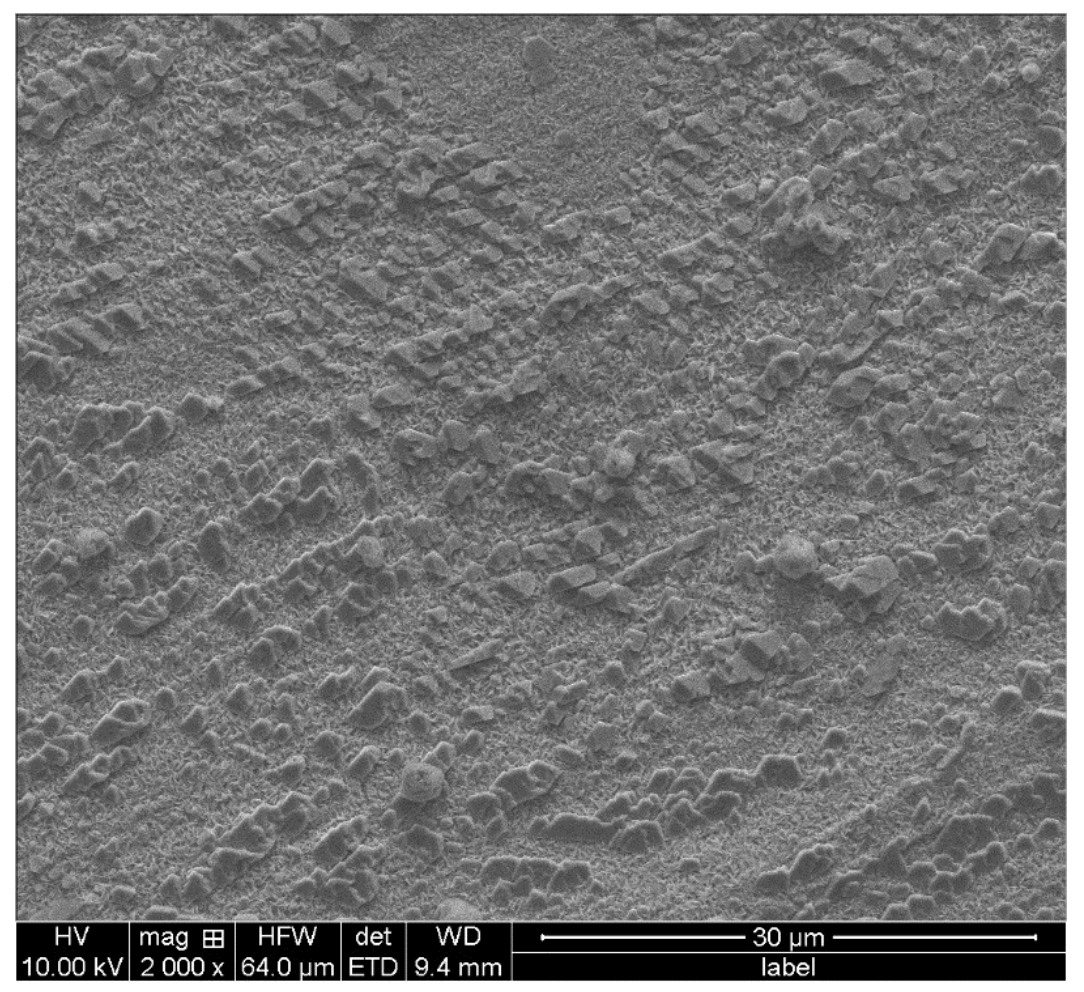

Figure 2.2. SEM image of a discolored "grey" patch on the oxidized copper foil.

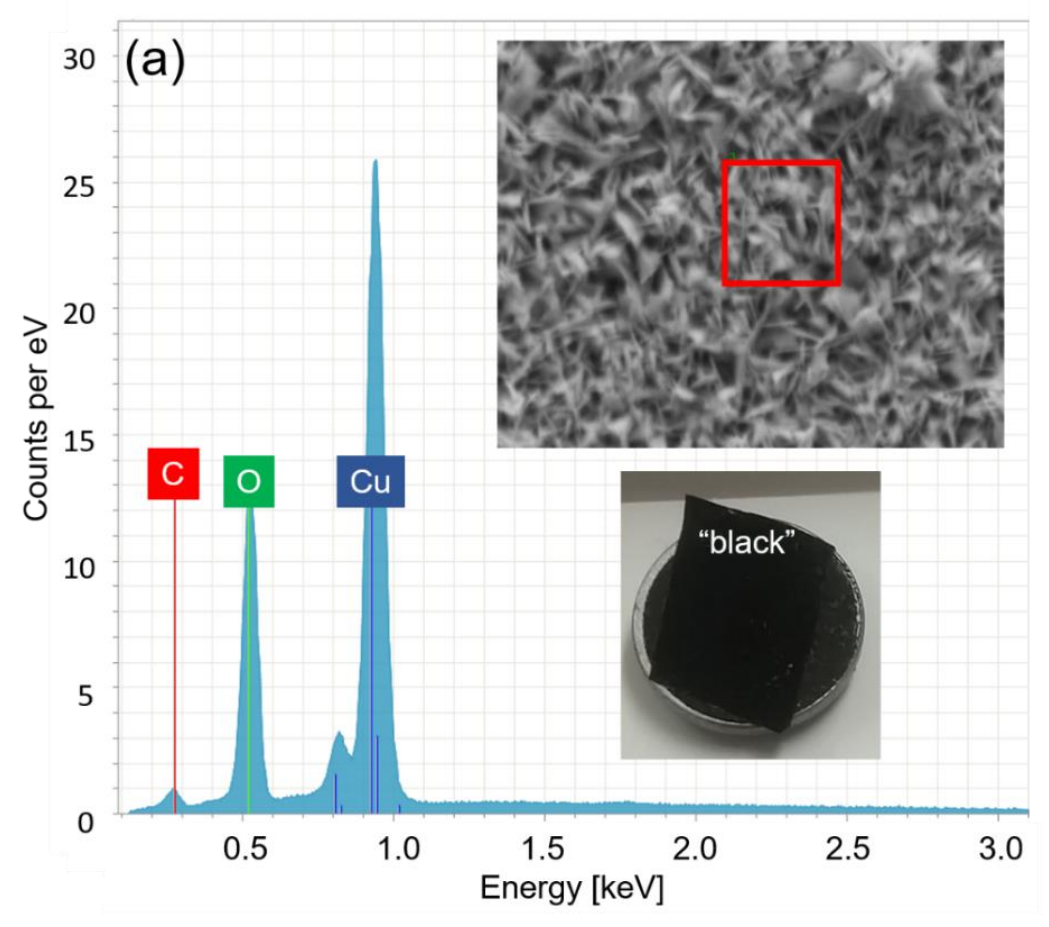




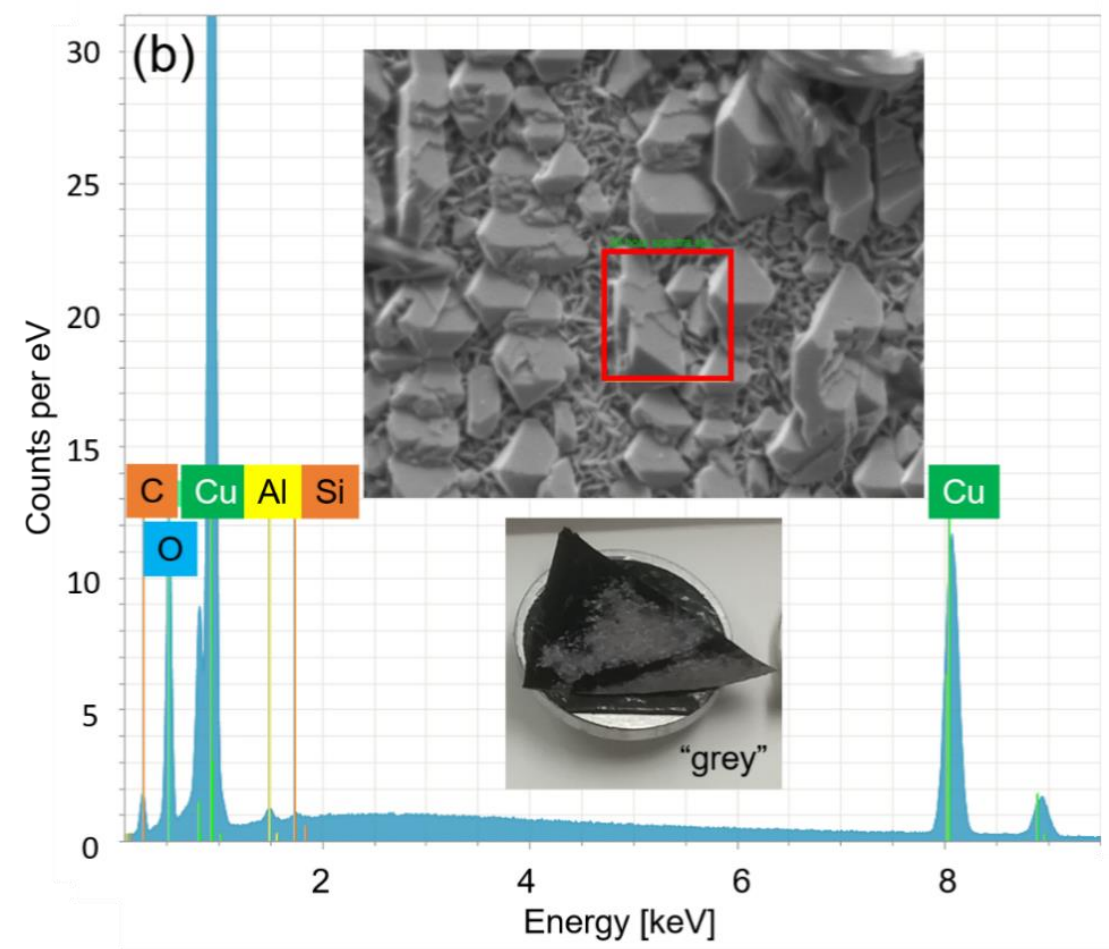

Figure 2.3. Energy-dispersive X-ray spectroscopy (EDS) scans of (a) black and (b) discolored regions of copper foil oxidized in an alkaline solution [98]. EDS was taken within the $\sim 3 \times 3 \mu \mathrm{m}^{2}$ boxes of the inset SEM images. Photos of each sample are inserted.

To address the nonuniformity of the $\mathrm{CuO}$ coverage, we used an alternative oxidation recipe by $\mathrm{Nam}$ and $\mathrm{Ju}[\mathbf{5 6}]$, which can produce $\mathrm{CuO}$ nanostructures on $\mathrm{Cu}$ foils with a similar "grass-like" morphology as the recipe by Liu et al. [98]. Commercially available copper foils (All Foils, Inc.) of thickness $12.7 \mu \mathrm{m}$ were cleaned in acetone for $\sim 20 \mathrm{~min}$, and the native oxide layer was removed in $2.0 \mathrm{M} \mathrm{HCl}$ solution for $\sim 30$ seconds. The foils were then quickly rinsed in deionized water and immersed in a hot alkaline bath at $95{ }^{\circ} \mathrm{C}$ consisting of $\mathrm{NaClO}_{2}, \mathrm{NaOH}, \mathrm{Na}_{3} \mathrm{PO}_{4} \cdot 12 \mathrm{H}_{2} \mathrm{O}$, and deionized water in the ratio 3.75:5:10:100 by weight. Immediately after immersion, the color of the foils transitioned from metallic pink to brown to black within a couple of minutes, which is significantly faster than the 20-hour oxidation process used previously [98]. After ten minutes in solution, the foils were rinsed with deionized water and air dried on a clean surface. 
Subsequent SEM imaging shows that ten minutes of oxidation leads to a uniform oxide layer on the surface of the copper foils. The two-step chemical reaction leading to the formation of $\mathrm{CuO}$ on $\mathrm{Cu}$ foil is described in Refs. [56-57]:

$$
\begin{gathered}
2 \mathrm{Cu}+2 \mathrm{OH}^{-} \rightarrow \mathrm{Cu}_{2} \mathrm{O}+\mathrm{H}_{2} \mathrm{O}+2 \mathrm{e}^{-} \\
\mathrm{Cu}_{2} \mathrm{O}+2 \mathrm{OH}^{-} \rightarrow 2 \mathrm{CuO}+\mathrm{H}_{2} \mathrm{O}+2 \mathrm{e}^{-}
\end{gathered}
$$

Note that the initially transparent solution changed to a greenish-blue color characteristic of copper-hydroxide $\left(\mathrm{Cu}(\mathrm{OH})_{2}\right)$ - after oxidizing a few foils, so a metastable intermediate like $\mathrm{Cu}(\mathrm{OH})_{2}$ might play a role in determining the final morphology of $\mathrm{CuO}$.

SEM images of oxidized foils are shown in Fig. $\mathbf{2 . 4}$ for samples taken out of solution at various times: $0 \mathrm{~s}$ (not exposed), $10 \mathrm{~s}, 30 \mathrm{~s}, 1 \mathrm{~min}, 2 \mathrm{~min}$, and $5 \mathrm{~min}$. Before exposure, the starting substrate (bare Cu foil) shows surface imperfections ("striations") caused by the manufacturing process, i.e., rolling. Upon exposure to the solution (Fig. 2.4b-e), the $\mathrm{Cu}$ surface rapidly becomes rougher with the development of oxide nanostructures. At 5 minutes (Fig. 2.4f), the foils are completely covered with a layer of nanostructures whose grass-like morphology is shown in the magnified SEM image in Fig. 2.5. From a survey of SEM images, the average dimensions of a triangular $\mathrm{CuO}$ blade are $2 \mu \mathrm{m}$ tall, $\sim 0.5 \mu \mathrm{m}$ wide at the base, and $\sim 20 \mathrm{~nm}$ thick. For the preparation of many (>10) samples, we note the following: (1) using a larger volume of solution yielded poorer results than for the $100 \mathrm{ml}$ bath size; (2) the need to exchange the $100 \mathrm{ml}$ solution after every $\sim 5$ foils due to the evaporation of water at $95^{\circ} \mathrm{C}$; and (3) the need to flip the foils after $\sim 5$ minutes or stir occasionally. 

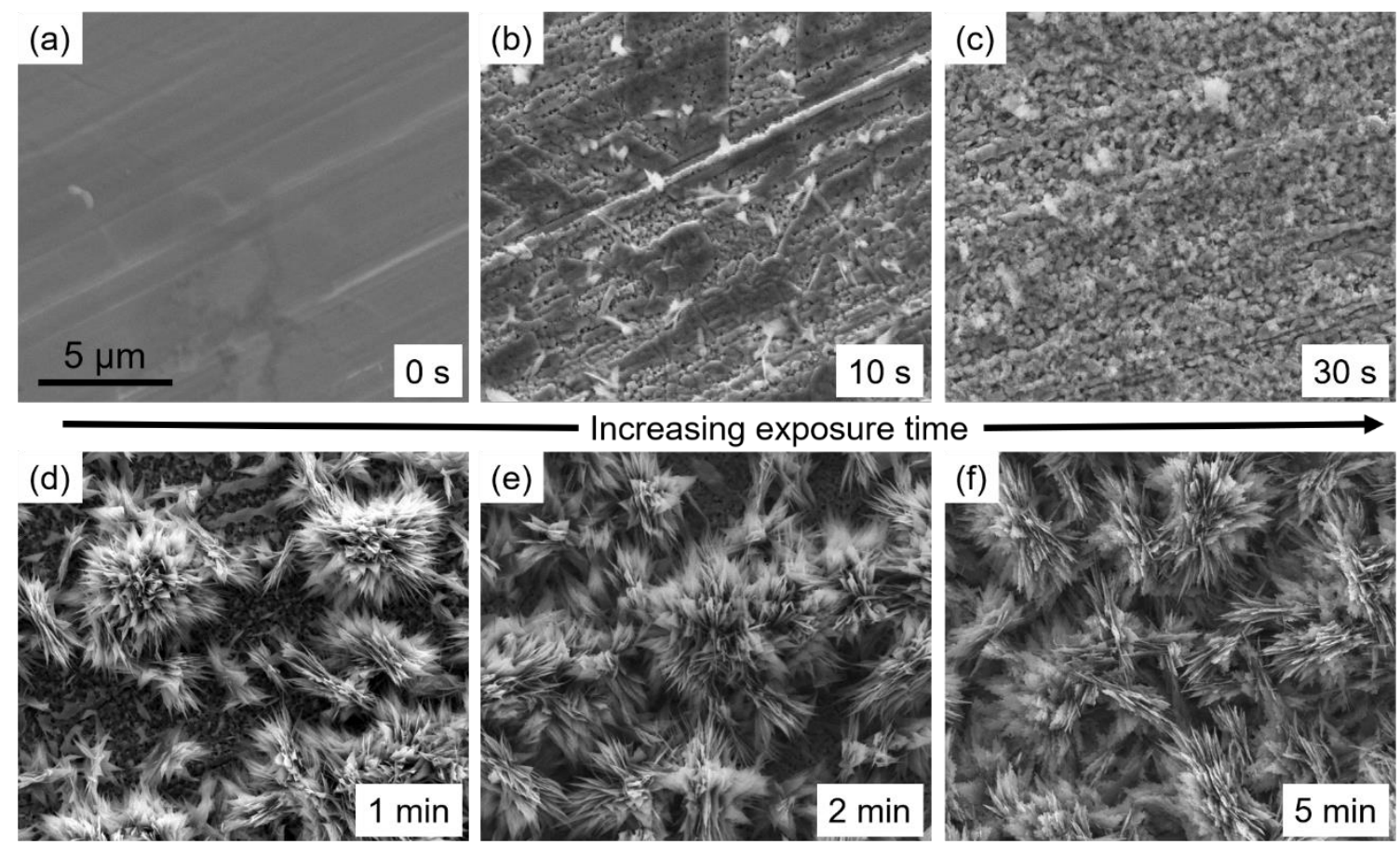

Figure 2.4. SEM images of a copper surface exposed to an oxidizing solution [56] for (a) $0 \mathrm{~s}$, (b) $10 \mathrm{~s}$, (c) $30 \mathrm{~s}$, (d) $1 \mathrm{~min}$, (e) $2 \mathrm{~min}$, and (f) $5 \mathrm{~min}$.

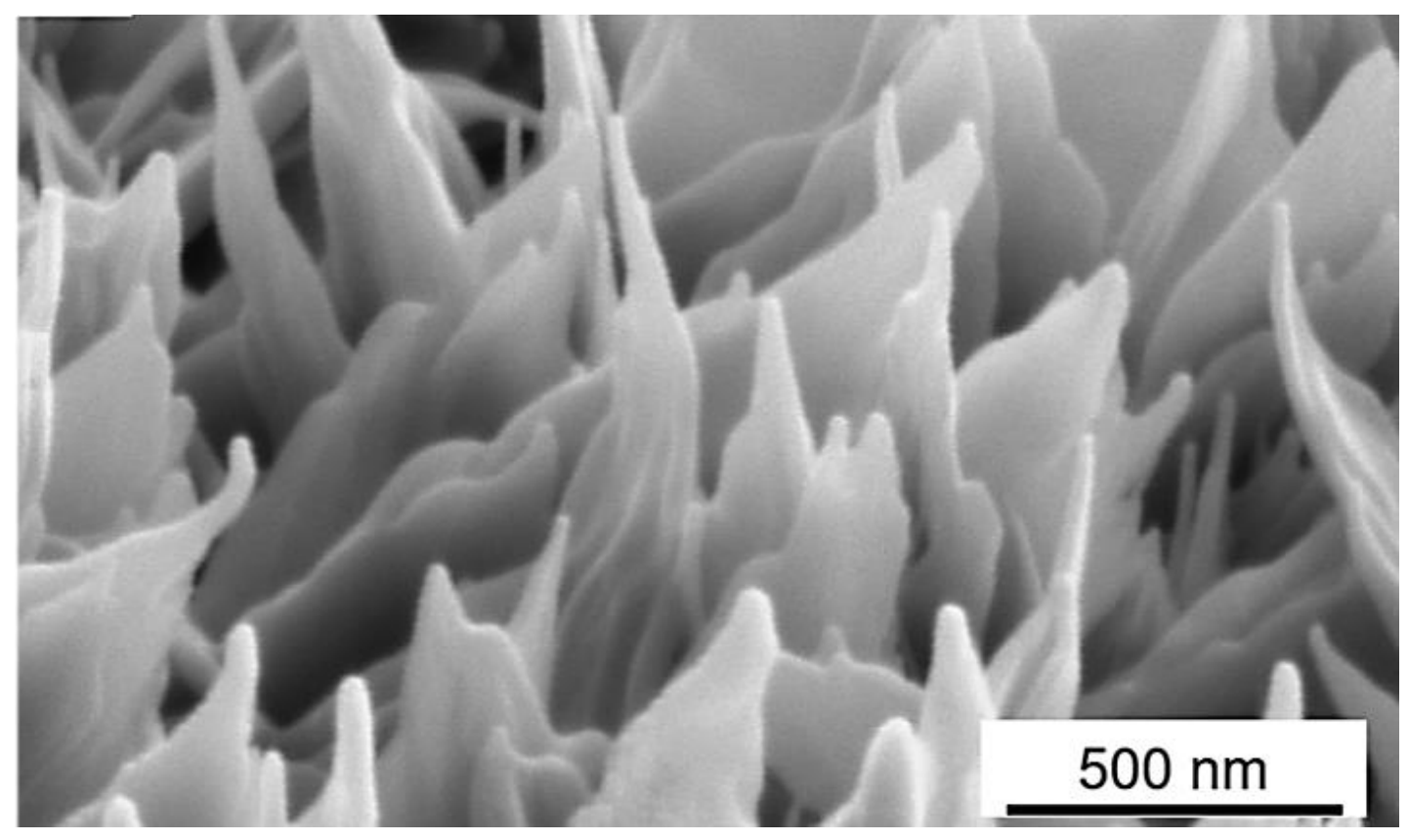

Figure 2.5. SEM image of the grass-like morphology of $\mathrm{CuO}$ nanostructures. 
The crystallinity, layered-structure, and chemical composition of the oxide layers were investigated using high-spatial-resolution transmission electron microscopy (TEM) at the MU Electron Microscopy Core Facility. The sample used for TEM characterization was prepared in a focused-ion beam (FIB)-SEM instrument (FEI Scios Analytical): a thin $(\sim 30 \mathrm{~nm})$ layer of platinum was deposited over a $25 \times 2.5 \mu \mathrm{m}^{2}$ rectangular area of the $\mathrm{CuO}$ surface to protect the nanostructures from damage during the ion-milling process. A highly energetic Ga-ion beam ( $5 \mathrm{kV}, 0.1 \mathrm{nA})$ was used for initial trenching, whose energy was subsequently lowered $(2 \mathrm{kV}, 16 \mathrm{pA})$ for final polishing. An SEM image of the sample after lift-out (prior to final polishing) is shown in Fig. 2.6.

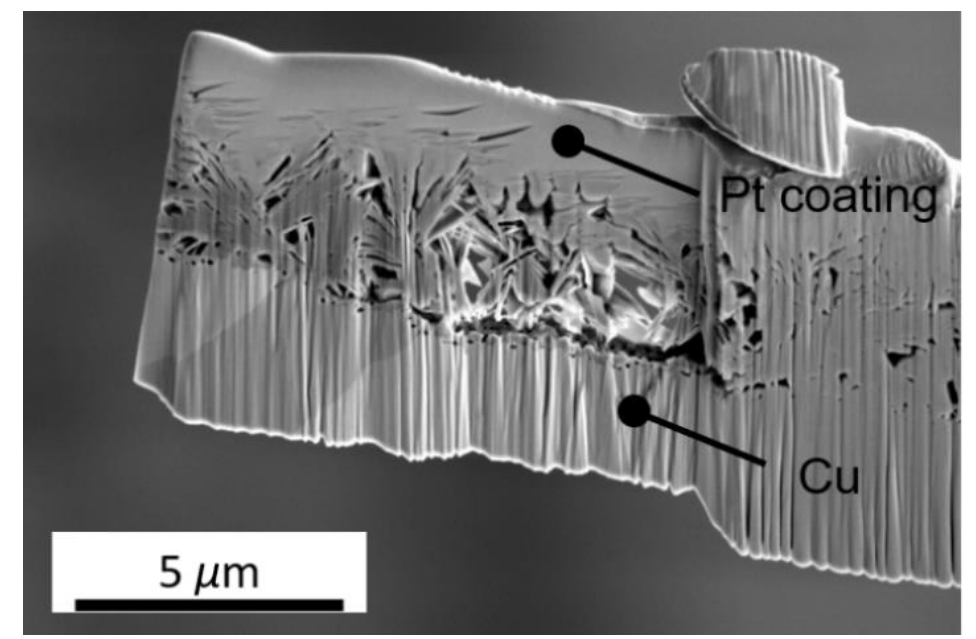

Figure 2.6. SEM image of a cross section of the sample used for TEM characterization.

The $~ 100 \mathrm{~nm}$-thick sample was transferred to a 300-kV TEM (FEI Tecnai Twin F30) for characterization. A cross-sectional view of a CuO-coated copper foil is shown in the high-angle annular dark-field (HAADF) image in Fig. 2.7a. HAADF images are formed primarily by Rutherford back-scattered electrons collected at relatively high angles with respect to the incident beam $(70 \mathrm{mrad}<\theta<400 \mathrm{mrad})$. In principle, the grey-scale intensity of an HAADF image is approximately proportional to $\mathrm{z}^{2}$ where $\mathrm{z}$ is 
the atomic number of the target atoms [101-102]. The average $\mathrm{z}^{2}$ values for the copper layers $\mathrm{Cu}, \mathrm{Cu}_{2} \mathrm{O}$, and $\mathrm{CuO}$ are approximately 841,484 , and 342 , respectively. Accordingly, the grey scale intensity of bulk $\mathrm{Cu}$ would be brightest, $\mathrm{CuO}$ the darkest, and $\mathrm{Cu}_{2} \mathrm{O}$ in between, consistent with the HAADF image contrast shown in Fig. 2.7a. The black areas in this figure are holes through which the incident electron beam passes unimpeded. The average thickness of the $\mathrm{Cu}_{2} \mathrm{O}$ layer normal to the $\mathrm{Cu}$ substrate is $216 \pm$ $98 \mathrm{~nm}$, which agrees with that measured by Enright et al. [57], although it is unclear how they distinguished the $\mathrm{Cu}_{2} \mathrm{O}$ layer and its thickness $(\sim 300 \mathrm{~nm})$ from $\mathrm{CuO}$ and $\mathrm{Cu}$ phases in their SEM images.

Selected-area electron diffraction (SAED) patterns were taken in the circled region in Fig. 2.7a. The pattern in Fig. 2.7b shows the resulting Bragg spots indicative of single-crystal domains [103], which could be indexed to $\mathrm{Cu}$ and $\mathrm{Cu}_{2} \mathrm{O}$ phases (see Table 2.1). Moreover, the SAED patterns revealed an epitaxial relationship between the grains of the $\mathrm{Cu}$ substrate and the $\mathrm{Cu}_{2} \mathrm{O}$ film: $\mathrm{Cu}[112]\left\|\mathrm{Cu}_{2} \mathrm{O}[112] ; \mathrm{Cu}(111)\right\| \mathrm{Cu}_{2} \mathrm{O}(111)$; and, $\mathrm{Cu}(220) \| \mathrm{Cu}_{2} \mathrm{O}(220)$ as has been observed previously [104-105].

\begin{tabular}{c|cccc}
\multicolumn{1}{c}{ Sample area } & Index & Obs. $\boldsymbol{d}[\AA]$ & Calc. $\boldsymbol{d}[\AA]$ & Diff. $[\%]$ \\
\hline \multirow{2}{*}{ Copper substrate } & $\mathrm{Cu}(200)$ & 1.821 & 1.807 & 0.77 \\
& $\mathrm{Cu}(111)$ & 2.089 & 2.087 & 0.01 \\
\hline \multirow{3}{*}{$\mathrm{Cu} / \mathrm{Cu}_{2} \mathrm{O}$ interface } & $\mathrm{Cu}(200)$ & 1.800 & 1.807 & 0.39 \\
& $\mathrm{Cu} 2 \mathrm{O}(111)$ & 2.474 & 2.465 & 0.37 \\
& $\mathrm{Cu}_{2} \mathrm{O}(200)$ & 2.120 & 2.135 & 0.70
\end{tabular}

Table 2.1. Comparison between observed and calculated $d$-spacings for Bragg spots indexed from SAED patterns of CuO-coated samples (see Fig. 2.7b). 

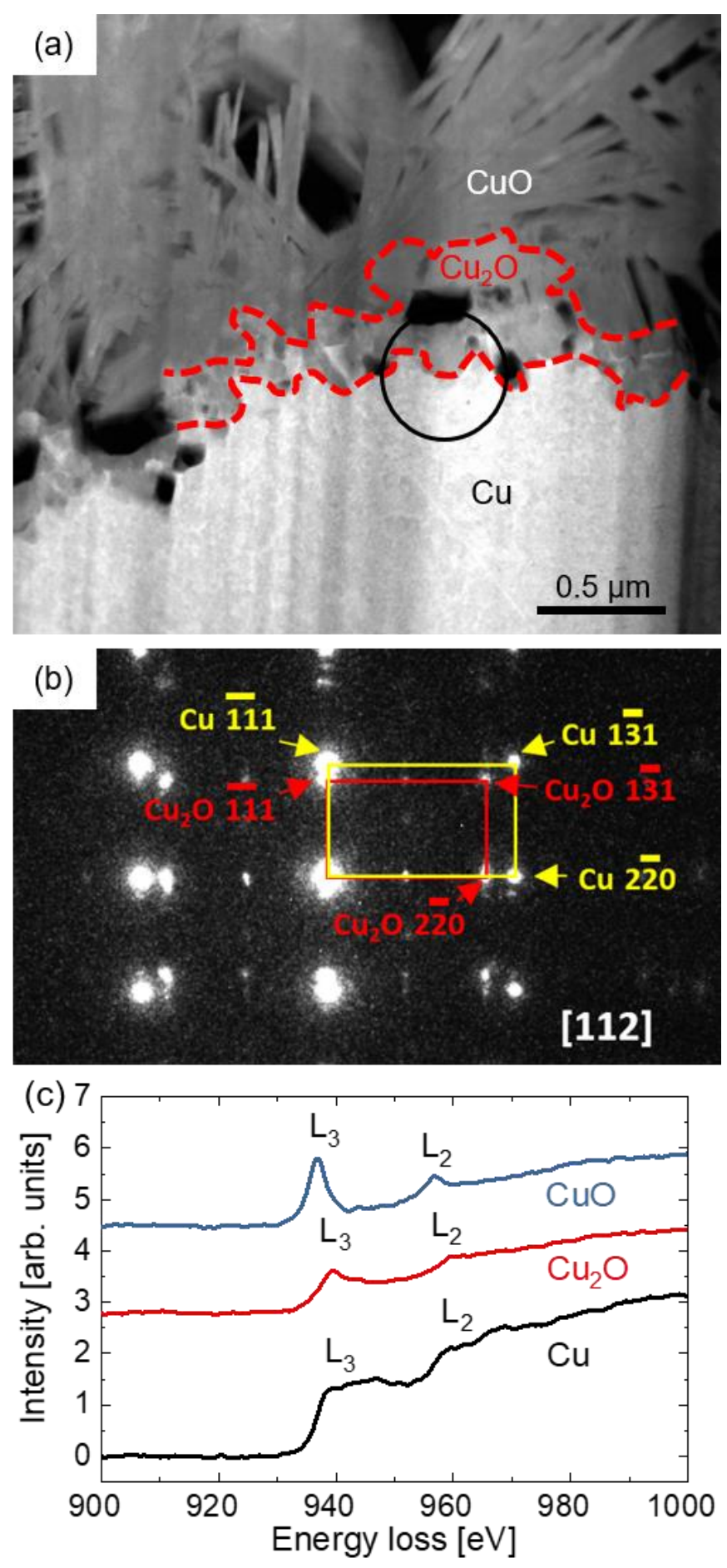

Figure 2.7. (a) HAADF image of a cross section of a chemically oxidized $\mathrm{Cu}$ foil. Layers $\left(\mathrm{Cu}, \mathrm{Cu}_{2} \mathrm{O}\right.$, and $\left.\mathrm{CuO}\right)$ are identified based on image contrast, enabling the $\mathrm{Cu}_{2} \mathrm{O}$ region to be outlined by the dashed red curve. The estimated thickness of the $\mathrm{Cu}_{2} \mathrm{O}$ layer is $216 \pm$ $98 \mathrm{~nm}$, in reasonable agreement with Ref. [57]. (b) SAED pattern taken in the circled region in (a) along the [112] zone axis of $\mathrm{Cu}$ and $\mathrm{Cu}_{2} \mathrm{O}$. (c) EELS scans taken within each layer of (a) with the copper $\mathrm{L}_{2}(951 \mathrm{eV})$ and $\mathrm{L}_{3}(931 \mathrm{eV})$ edges labeled. Spectra are offset vertically for clarity. 
Electron energy-loss spectroscopy (EELS) scans collected simultaneously with TEM images were used to determine chemical composition in the layers identified by image contrast in Fig. 2.7a. Representative EELS scans are shown in Fig. 2.7c where the energy-resolution is $\sim 1 \mathrm{eV}$ based on the width of the zero-loss peak. The background was removed using standard analysis methods available in the acquisition software. In these scans, the incident electron beam $(300 \mathrm{keV})$ excites the sample's electrons into vacant

states above the ionization edges [106]. The shapes of the L-transitions (2p-to-3d shells) near $931 \mathrm{eV}$ and $951 \mathrm{eV}$ and their intensity ratio $\left(\mathrm{L}_{3}-\mathrm{to}_{\mathrm{L}} \mathrm{L}_{2}\right)$ can be used to fingerprint the oxidation state of copper [107]. The shapes of the EELS edges agree well with those reported elsewhere [108]; and, together with the HAADF images and SAED patterns, confirm the identities of the layers labeled in Fig. 2.7a.

\subsection{Hydrophilicity and Wetting Behavior}

Micro- and nano-structuring of surfaces can enhance their wetting properties $[\mathbf{3 4 , 5 5 , 5 9 ]}$ as was found for the $\mathrm{CuO}$ coatings used in FP-OHPs [56,98]. As discussed in Sec. 1.3, the most common test to determine hydrophilicity is by a water contact-angle measurement. Our measurements were all conducted in air using $1 \mu 1$ droplets of purified water (Ramé-Hart model 200), and our results show that bare copper is weakly hydrophilic/hydrophobic with a contact angle of $\sim 70-90^{\circ}$ (see Fig. 2.8a). In contrast, water deposited on the $\mathrm{CuO}$ surface readily wets the nanostructures and produces a vanishing $\left(\sim 0^{\circ}\right)$ contact angle (Fig. 2.8b), which is characteristic of superhydrophilic surfaces [34]. 

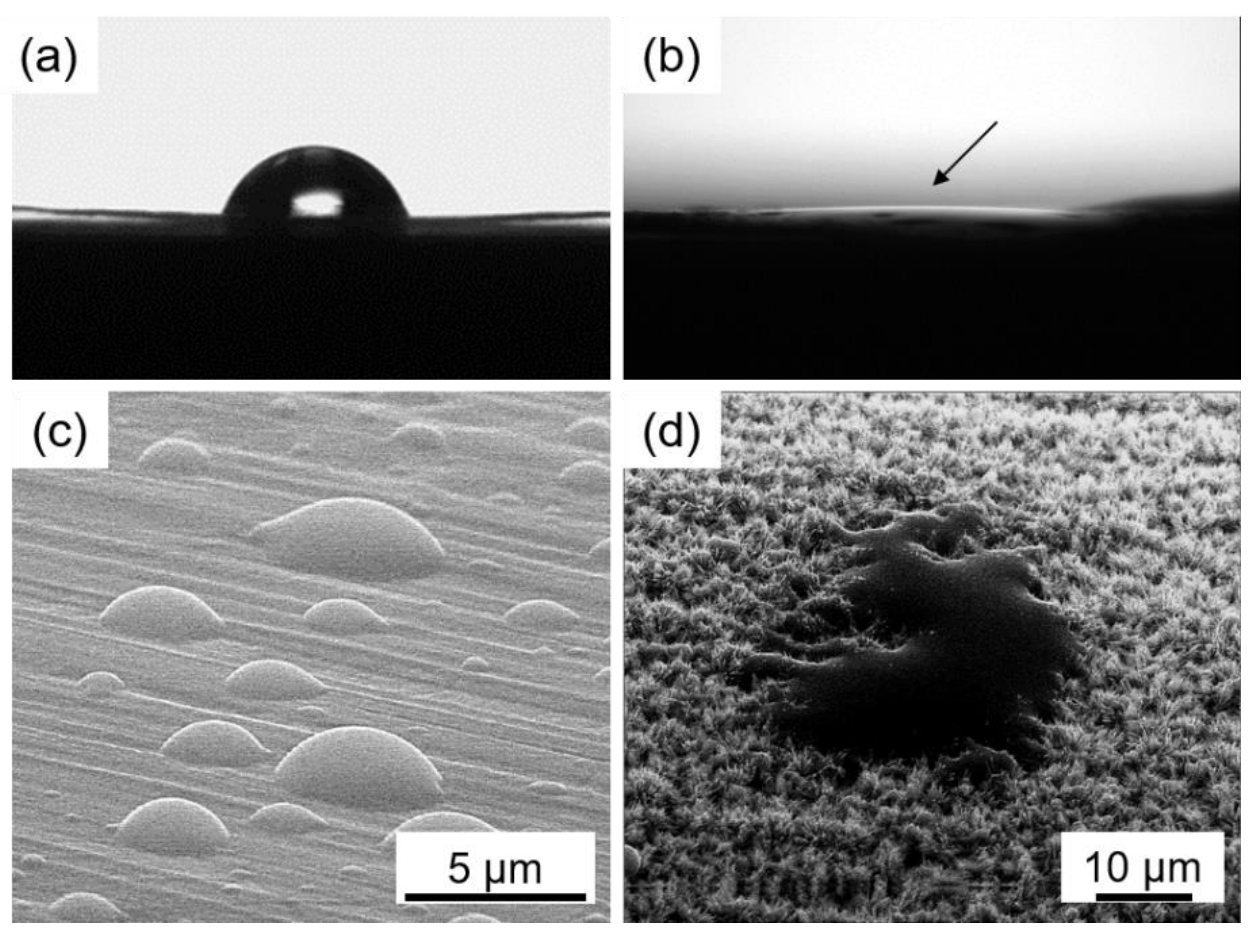

Figure 2.8. Equilibrium water contact-angle measurements, using $1 \mu 1$ droplets, for (a) bare- $\mathrm{Cu}$ and (b) $\mathrm{CuO}$-coated $\mathrm{Cu}$ surfaces with average values of $70^{\circ}$ and $\sim 0^{\circ}$, respectively. The arrow in (b) points to the water droplet that has spread out on the $\mathrm{CuO}$ surface. (c-d) Observation by ESEM of water condensation on (c) untreated-Cu and (d) $\mathrm{CuO}$ surfaces.

In addition to water contact-angle evaluation, we used environmental-SEM (ESEM) water-condensation measurements to elucidate the differences in wetting on untreated- $\mathrm{Cu}$ and $\mathrm{CuO}$-coated $\mathrm{Cu}$ foils at the microscale. ESEM is an operational mode in which water vapor (or other gas) is introduced into the SEM chamber at relatively low pressures (2600 Pa), enabling the study of biological or insulating surfaces without invasive sample preparation procedures [109]. We take advantage of ESEM by creating a humid environment around the sample similar to that within our neutron scattering sample cell (see Appendix A). In addition, the sample temperature can be controlled to induce condensation or evaporation of liquid water, allowing us to determine wetting behavior and water droplet morphology on the micron scale. 
To observe the wetting of water to $\mathrm{CuO}$ blades, an $\sim 8 \times 13 \mu \mathrm{m}^{2}$ rectangular trench was milled into the surface of a $\sim 1 \times 1 \mathrm{~cm}^{2} \mathrm{CuO}$-coated $\mathrm{Cu}$ foil using a focusedion beam (FIB) in a FIB-SEM instrument (FEI Scios Analytical). The sample was then mounted on a custom-made oxygen-free high-conductivity (OFHC) copper sample stub, titled at an angle of $\sim 15^{\circ}$ relative to the incident beam direction in order to minimize beam heating effects [110-111]. High-thermal-conductivity paste (Arctic Silver 5) was used to provide optimal thermal contact between the sample and the Peltier cold-stage. ESEM images were obtained by collecting electrons in a gaseous backscattered-electron detector as the incident electron beam rasters across the sample's surface. Image recording ended when the water layer thickness exceeded the escape length of backscattered electrons, as indicated by the total loss of image contrast. Reproducibility was confirmed by repeating condensation and evaporation cycles on several samples.

The dew point near the sample surface was controlled by adjusting the vapor pressure of the SEM chamber $(0-800 \mathrm{~Pa})$ as well as the sample temperature $(268-298 \mathrm{~K})$. In addition, we adjusted the electron beam accelerating voltage $(5-20 \mathrm{kV})$, sampledetector gap distance $(10-15 \mathrm{~mm})$, and image dwell time $(0.3-30 \mu \mathrm{s})$ for the best signalto-noise ratio. We found that a water vapor pressure of $~ 500 \mathrm{~Pa}$ provided a good compromise between humidity and image noise. The predicted saturated vapor pressure (dew point) $e_{s}$ over water was determined using Tetens' (empirical) formula [112],

$$
e_{S}=e_{0} \exp \left[\frac{b\left(T-T_{1}\right)}{T-T_{2}}\right]
$$

where $e_{0}$ is the saturated vapor pressure at $273.16 \mathrm{~K}$ approximately equal to $611 \mathrm{~Pa} ; b$ is a constant (17.27); and, $T_{1}$ and $T_{2}$ are equal to $273.16 \mathrm{~K}$ and $35.86 \mathrm{~K}$, respectively. 
Choosing $e_{s}$ of $500 \mathrm{~Pa}$, the calculated dew point is $\sim 270.44 \mathrm{~K}$ or $-2.72{ }^{\circ} \mathrm{C}$, which can be achieved using the Peltier cold-stage accessory of the SEM instrument (FEI Quanta 600F). Controlling the sample temperature in this way allowed us to initiate condensation and evaporation of water similar to the technique used by others [113].

The water condensation procedure begins by equilibrating the sample at a temperature just above the dew point (e.g., $273 \mathrm{~K}$ ) for 5 minutes. Then, the sample is cooled in steps ranging from $0.1 \mathrm{~K}$ to $1 \mathrm{~K}$ at the slowest allowable temperature ramping rate of $1 \mathrm{~K} / \mathrm{min}$ and equilibrated for at least one minute after each temperature change. At the dew point, the system is in equilibrium where the number of water molecules that condense is equal to that which evaporates. Just below the dew point, around $269.5 \mathrm{~K}$, ESEM imaging shows the onset of condensation. Water droplets nucleate and grow rapidly over a span of a couple minutes during which video is recorded. After a few minutes, the $\mathrm{CuO}$ surface becomes completely submerged, and droplets grow larger than the image frame size, prompting the end of image recording.

Here, we comment on the state of water on the $\mathrm{CuO}$ surface during this experiment. The conditions under which condensation occurs in these experiments (269.5 $\mathrm{K}, 500 \mathrm{~Pa}$ ) appear to cross the solid-vapor coexistence line just below the triple point as indicated by the dashed blue line in Fig. 2.10. To reconcile this issue, we first note that condensation is a non-equilibrium thermodynamic process and therefore the $P T$-phase diagram in Fig. 2.10 does not accurately reflect the state of the water on the $\mathrm{CuO}$ surface during condensation [113]. Furthermore, our analysis of water wetting to $\mathrm{CuO}$ is based on images of small ( $<100 \mu \mathrm{m}$ diameter) droplets, which are known to supercool due to finite size effects [114]. Based on image contrast alone, we cannot rule out the possibility 
of the deposition or growth of solid water on the $\mathrm{CuO}$ surface; however, comparing our ESEM images with those of bulk ice at similar conditions [113], the droplets in our system have rounded shapes and tend to coalesce, suggesting the liquid state. To pursue this point, we can apply the Young-Laplace equation, which expresses the pressure discontinuity $\Delta P$ at a curved interface [113],

$$
\Delta P=P_{w}-P_{E S E M}=\frac{2 \gamma_{l v}}{R}
$$

Here, $R$ is the droplet radius $([\mu \mathrm{m}])$, and the subscripts $w$ and ESEM refer to the pressure within the water droplet and ESEM chamber, respectively; $\gamma_{l v}$ is the liquid-vapor surface tension of supercooled water, which is approximately $76.5 \mathrm{kPa} / \mu \mathrm{m}$ at $-3{ }^{\circ} \mathrm{C}$ [115]. Using a droplet radius of $2 \mu \mathrm{m}$ as an example (circled droplet Fig. 2.9), the droplet's internal pressure $P_{w}$ is approximately $76.5 \mathrm{kPa}$. This value suggests the water is effectively in the liquid region of the phase diagram [116] depicted by the red asterisk (*) in Fig. 2.10. While this argument can explain the observation of liquid water in our ESEM images, we must emphasize that the phase diagram is applicable to macroscopic systems in thermodynamic equilibrium [113].

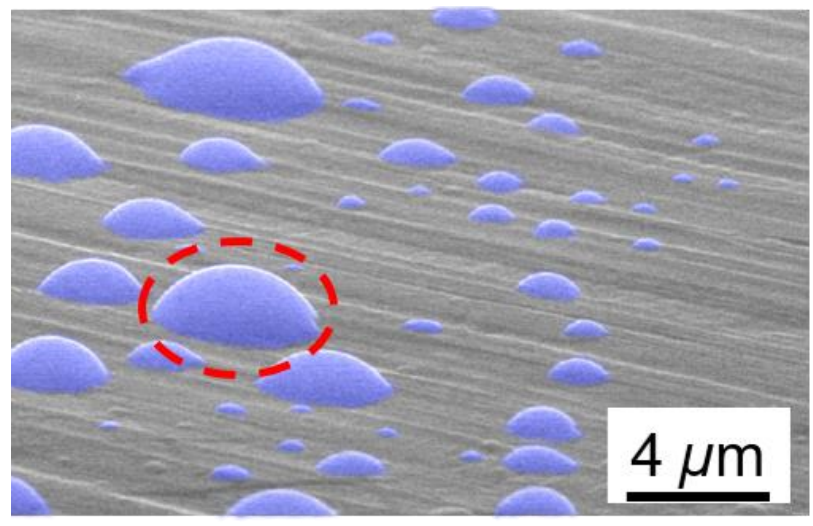

Figure 2.9. ESEM image of water condensed on the surface of a bare-Cu foil. The Young-Laplace pressure (Eq. (2.4)) was evaluated for the circled droplet. 


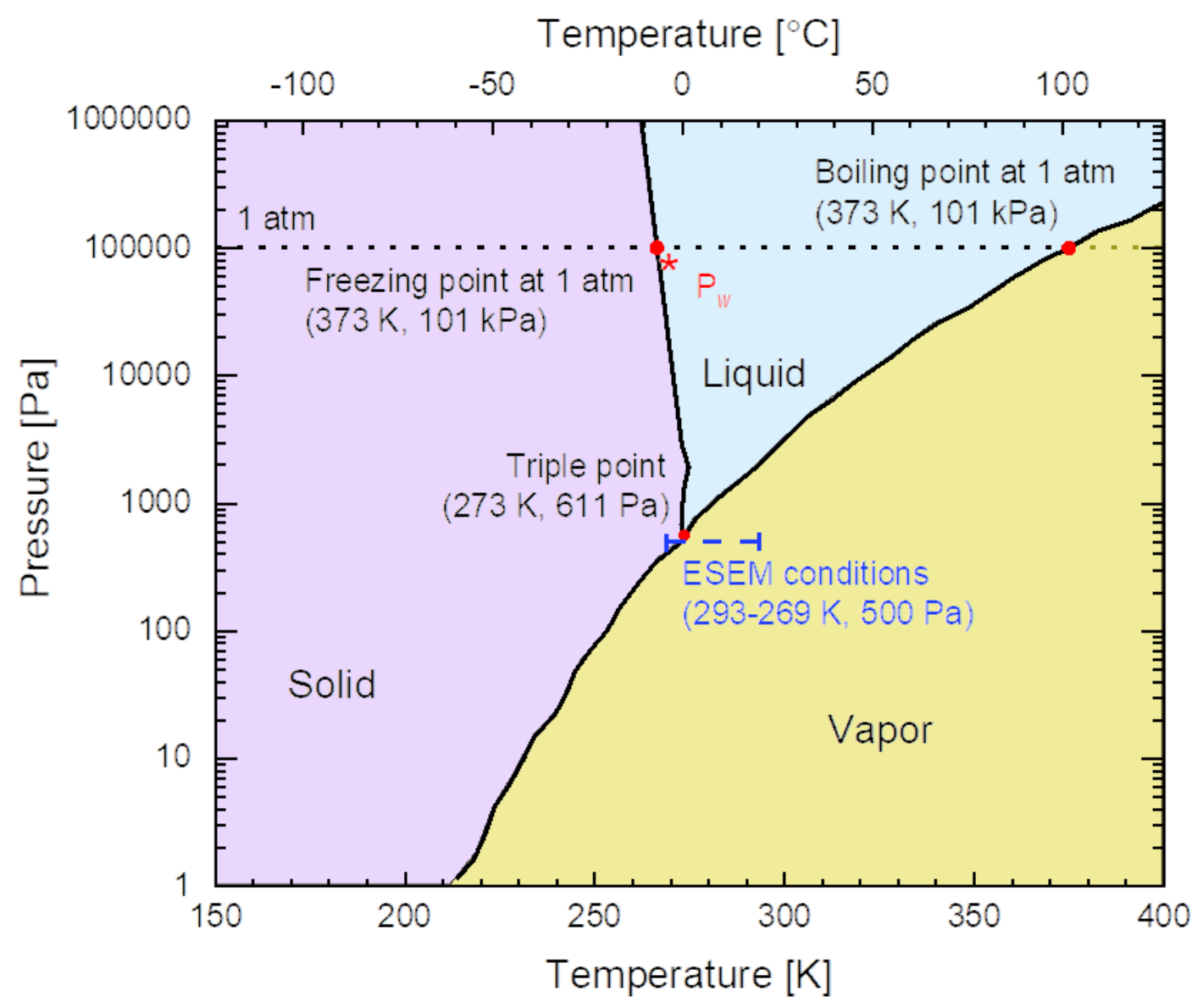

Figure 2.10. Equilibrium phase diagram of bulk water. Adapted from [116] (original available from: http://math.ucr.edu/home/baez/chemical/725pxPhase_diagram_of_water.svg.png). Temperature and pressure conditions of our ESEM experiments are shown as the dashed horizontal line near the triple point. The YoungLaplace pressure $P_{w}$ for a water droplet of radius $2 \mu \mathrm{m}$ (see Eqn. (2.4)) is $\sim 76.5 \mathrm{kPa}$ at $\sim 269.5 \mathrm{~K}$, shown as the red asterisk (*) just within boundary of the liquid phase.

A summary of our ESEM results for bare- $\mathrm{Cu}$ and $\mathrm{CuO}$ samples is shown in Fig.

2.8c and Fig. 2.8d, respectively. On the bare $\mathrm{Cu}$ surface, we observed hemisphericallyshaped droplets that nucleated along the striations of the $\mathrm{Cu}$ surface. Video playback (select frames are given in Fig. 2.11(a-c)) shows that surface wetting proceeds in a twostep process: the droplet volume increases slightly, as seen by an increase in height, followed by the liquid front moving outward radially, following the striations of the copper foil. Throughout the growth period, the droplets retained their hemispherical shape in qualitative agreement with water contact angle measurements (Fig. 2.8a). 
In contrast, condensed water on the $\mathrm{CuO}$ coating nucleated near the base of the nanostructures and proceeded to fill the pore space from valley to peak (see Fig. 2.11(ef), Fig. 2.12(b-c), and Fig. 2.13(a-c)). Simultaneously, the three-phase (liquid, vapor, solid) contact line expanded laterally such that water tended to fill the pore space between neighboring $\mathrm{CuO}$ blades, again, from valley to peak (see Fig. 2.13(a-c)). At this stage, ESEM images in Fig. 2.11 show liquid water wetting to the $\mathrm{CuO}$ blades, forming a weblike morphology as in the Wenzel wetting model [35]. After filling a pore between $\mathrm{CuO}$ blades, the water began to form a hemispherical droplet shape with its edges pinned to the CuO blades (see Fig. 2.13(d)). As the droplets grew, we saw that their contact line was pinned by the nanostructures (see Fig. 2.13(e-h)).
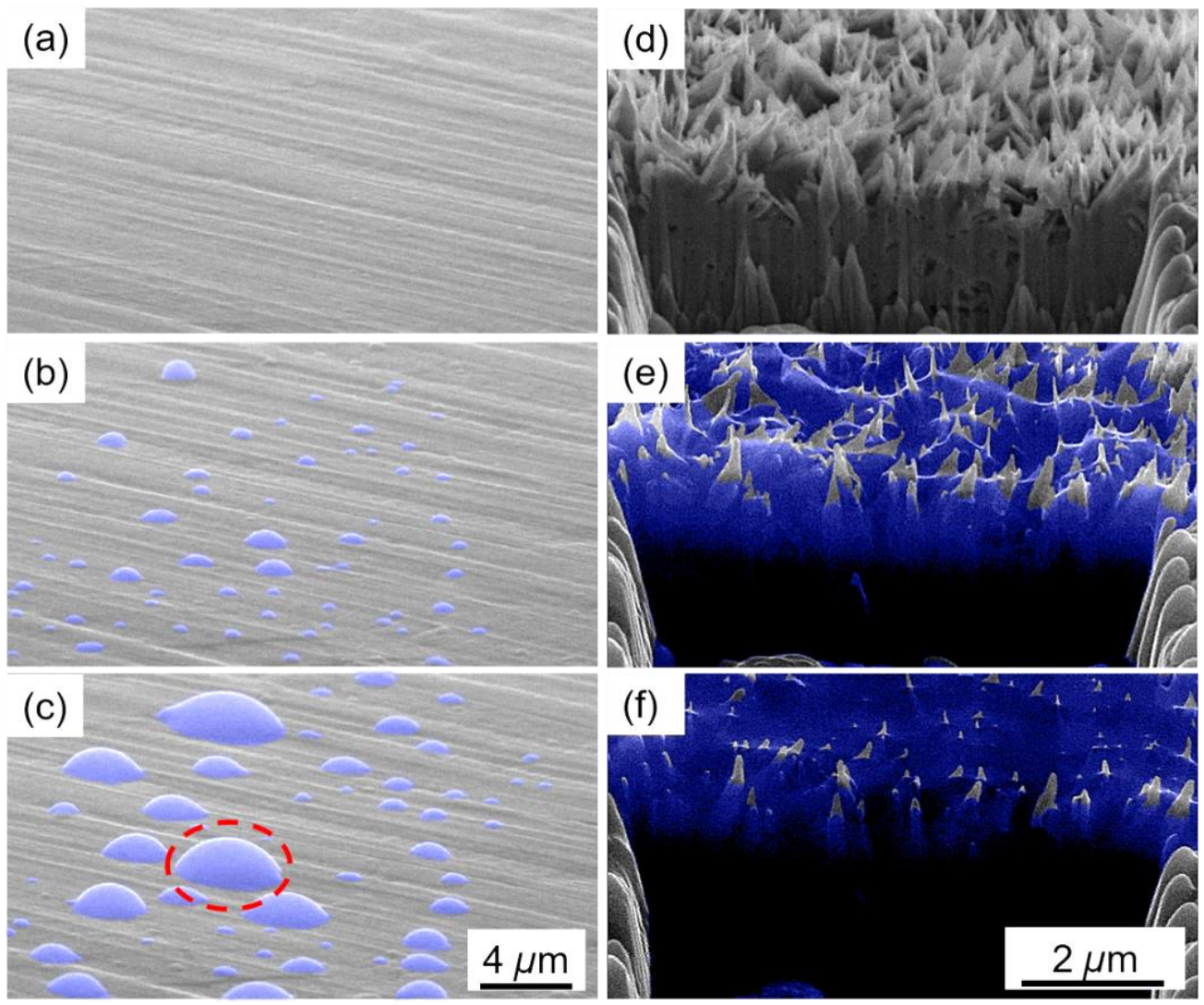

Figure 2.11. False-colored ESEM images of water actively condensing on bare $\mathrm{Cu}$ (a-c) and $\mathrm{CuO}$ nanostructures (d-f). The colored pixels identified as water are discriminated based on image contrast and intensity relative to the dry state in (d). 


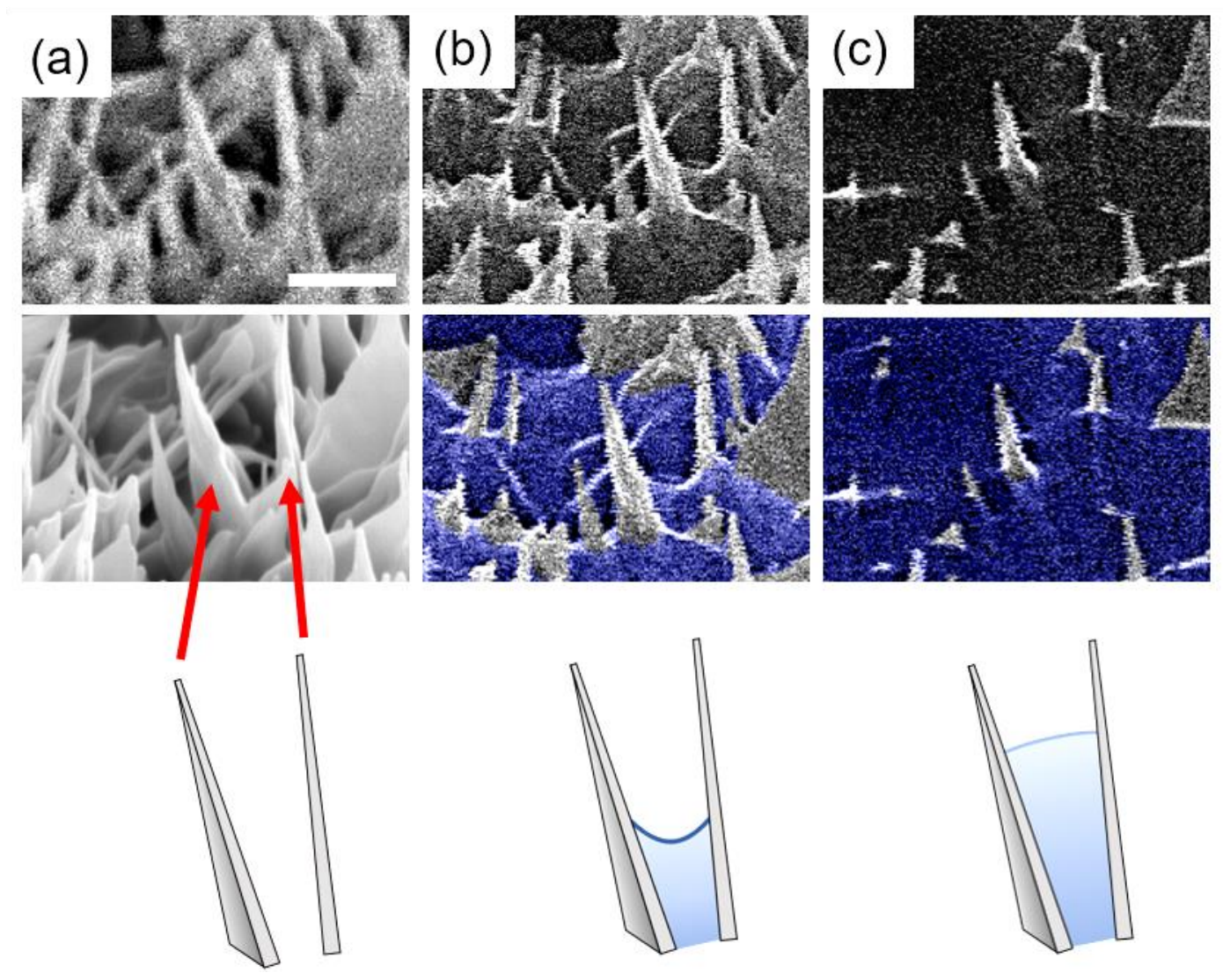

Figure 2.12. Magnified ESEM images of the dry (a) and wet (b-c) $\mathrm{CuO}$ surfaces previously shown in Fig. 2.11(d-e). Liquid water was identified by image contrast and by comparing with the initially dry condition shown in (a). In the image, water was colored blue color using computer software (Adobe Photoshop). Cartoon illustrations of two adjacent $\mathrm{CuO}$ blades are shown to highlight the water meniscus and pore-filling (i.e., the Wenzel state [35]). Scale bar is $0.5 \mu \mathrm{m}$. 

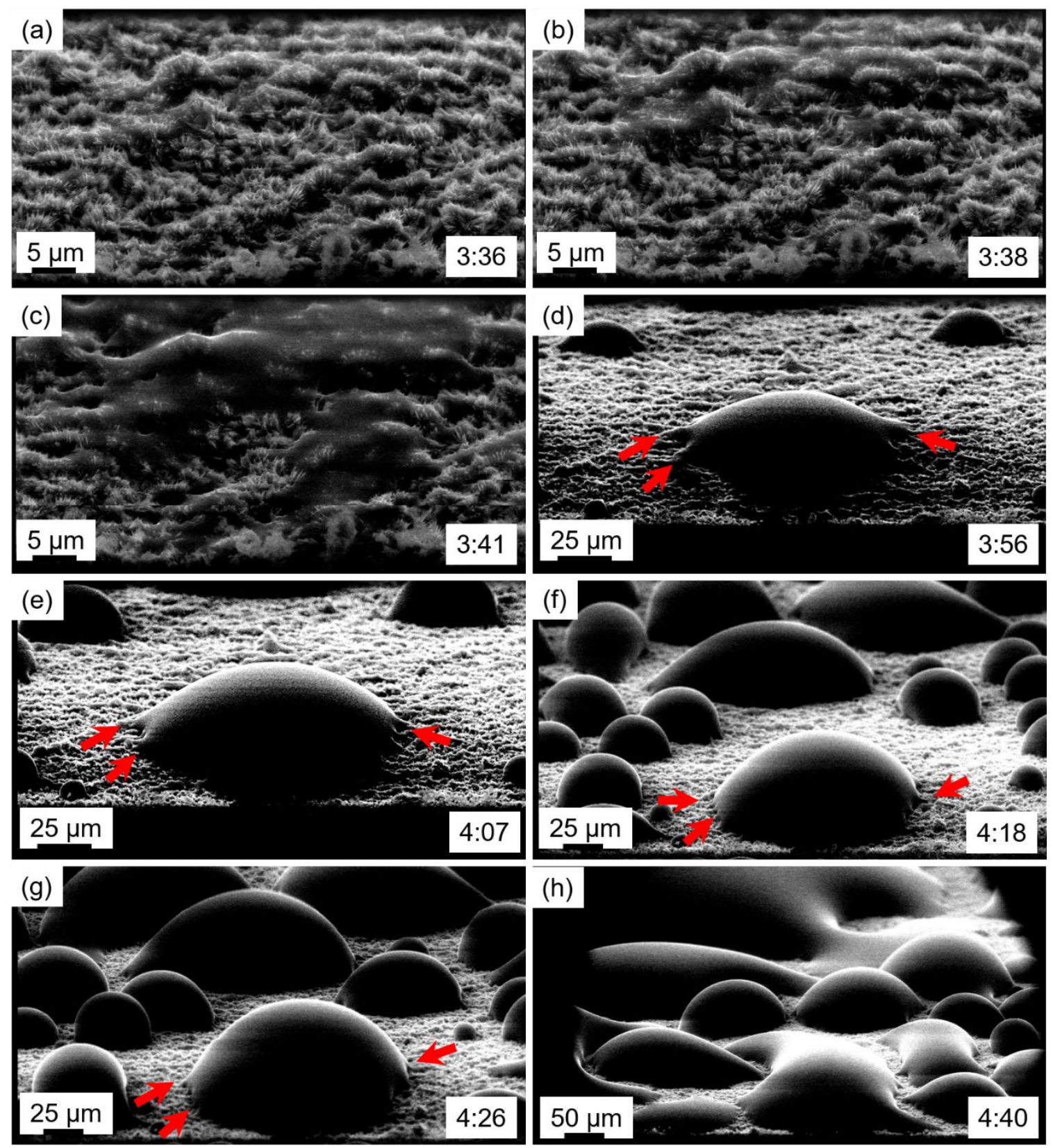

Figure 2.13. ESEM video screenshots of water condensing on a $\mathrm{CuO}$-coated $\mathrm{Cu}$ foil at multiple length scales. Timestamps (min.: sec.) are given in the bottom right corner of each image. (a-c) Initial stages of condensation, shortly after nucleation at the base of the $\mathrm{CuO}$ nanostructures. Water fills the funnel-shaped pores vertically (valley-to-peak) and expands laterally, filling adjacent pores. (c-g) When a pore is filled, a hemispherical droplet forms above it with its three-phase contact line being pinned to nanostructures (red arrows). (h) Eventually, droplets coalesce, forming different morphologies. 


\subsection{Surface Area, Porosity, and Sorption Properties of the Nanostructured $\mathrm{CuO}$ Coating}

The porosity of the nanostructured $\mathrm{CuO}$ coating was studied using nitrogen $\left(\mathrm{N}_{2}\right)$ isotherms measured on a Quantachrome Autosorb instrument after outgassing the sample at $513 \mathrm{~K}$ for 20 hours. Figure 2.14a shows the $\mathrm{N}_{2}$ adsorption and desorption isotherms for a $\sim 0.87 \mathrm{~g}$ sample of $\mathrm{CuO}$-coated $\mathrm{Cu}$ foils (four 5-cm-diameter foils). The mass of these foils also includes that of the copper substrate, which is nominally $10 \mu \mathrm{m}$ thick for each foil. By removal of the oxide coating using diluted-HCl, the mass of the metallic copper was about $0.61 \mathrm{~g}$; so, the $\mathrm{CuO}$ coating accounts for $\sim 0.26 \mathrm{~g}$ of the sample mass used for these nitrogen isotherm experiments. The inset shows the pore-size distribution calculated by the BJH method [117] (embedded in the software of the Quantachrome instrument), which indicates that the $\mathrm{CuO}$ coating contains both micro- and mesopores

with pore sizes in the ranges $0-20 \AA$ and $20-500 \AA$, respectively [118]. The wide range of pore sizes probed by $\mathrm{N}_{2}$ molecules is consistent with the random spacing between $\mathrm{CuO}$ blades observed in our electron microscope images (see, e.g., Fig. 2.5 and Fig. 2.7a).

It is clear in Fig. 2.14a that the $\mathrm{CuO}$ coating readily uptakes $\mathrm{N}_{2}$, based on the sharp increase in the amount adsorbed at low pressure $\left(P / P_{0} \rightarrow 0\right)$. On increasing the pressure, one sees a number of steps corresponding to layer-by-layer adsorption of $\mathrm{N}_{2}$. In the IUPAC system [118], the data closely resemble that of type-VI adsorption in which the step-height represents the adsorption capacity of each wetting layer and the sharpness of each step depends on the temperature and the interaction potential between the probe gas molecules and the atoms of the substrate [119]. The rare type-VI isotherm has been 
observed in the adsorption of krypton (at $90 \mathrm{~K}$ ) on graphitized carbon black, which possesses a highly uniform surface [119]. A characteristic feature of type-VI isotherms is that they are reversible, which does not appear to be the case in the $\mathrm{CuO}$ system given the hysteresis in the desorption branch (open points in Fig. 2.14a). Furthermore, the desorption data display an interesting behavior where, at certain pressures $(\sim 0.8,0.55$, and 0.3), it appears that some of the $\mathrm{N}_{2}$ molecules release from the surface/pores and give rise to local maxima and minima. These sorption phenomena have been observed previously in systems consisting of niobium-doped (0-1 \%) tungsten-oxide $\left(\mathrm{WO}_{3}\right)$ nanoparticles [120]; however, the origin of the unusual desorption behavior in both systems remains to be determined.

The specific surface area of the $\mathrm{CuO}$ coating was estimated by the BET method [121] ( Brunauer, Emmett, and Teller) to be $\sim 0.6 \mathrm{~m}^{2}$ per gram of $\mathrm{CuO}$-coated $\mathrm{Cu}$ foils, which includes the relatively large mass of the copper substrates. An equivalent treatment of bare-Cu foils (not shown) yields $\sim 0.1 \mathrm{~m}^{2} / \mathrm{g}$. We recognize that these measurements lie at the lower end of the instrument sensitivity, and we addressed this issue by reproducing the low specific surface area of $\sim 0.54 \mathrm{~g}$ sample of exfoliated graphite (i.e., Grafoil@) of $22.4 \mathrm{~m}^{2} / \mathrm{g}$. Assuming that the results of our $\mathrm{N}_{2}$ sorption measurements accurately reflect the filling of $\mathrm{CuO}$ pores, the cumulative adsorption of $\mathrm{N}_{2}$ is plotted versus pore size in Fig. 2.14b. 

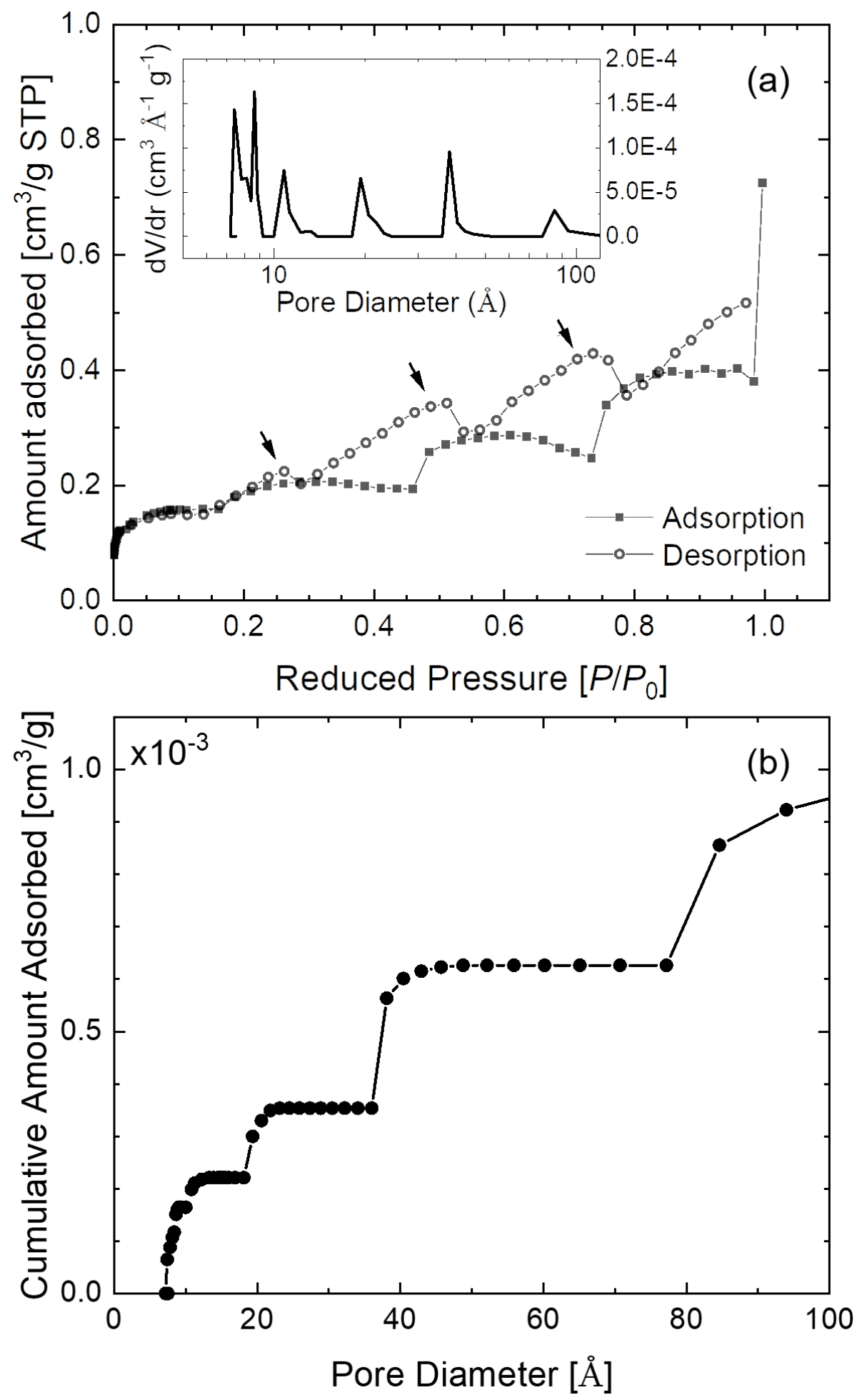

Figure 2.14. (a) Vapor isotherms for adsorption (closed squares) and desorption (open circles) of $\mathrm{N}_{2}$ as a function of reduced pressure $\left(P / P_{0}\right)$ at $77 \mathrm{~K}$. Arrows point to artifacts in the desorption data (see text). (Inset) Pore-size distribution determined by the BJH method [117]. (b) Plot of the cumulative amount of $\mathrm{N}_{2}$ adsorbed by $\mathrm{CuO}$-coated samples vs. pore diameter. 
Nitrogen is a weakly interacting molecule that does not participate in hydrogen bonding with the surface as do water molecules on the superhydrophilic $\mathrm{CuO}$ nanostructures. $\mathrm{H}$-bonding between water and $\mathrm{CuO}$ is discussed in Sec. 4.1, which contains measurements of the vibration spectrum of ice by inelastic neutron scattering. Therefore, a water sorption measurement was also performed on a $\sim 0.39$-gram sample of CuO-coated copper foil using a BELSORP-max instrument at $298 \mathrm{~K}$ after outgassing the sample at $373 \mathrm{~K}$ for 24 hours. The adsorption and desorption isotherms are shown in Fig. 2.15. In the IUPAC system [118], the adsorption follows that of a type-III isotherm characterized by no identifiable monolayer formation. The increasing slope up to the saturated vapor pressure $\left(P_{0}\right)$ indicates capillary condensation, and hysteresis might be attributed to metastability of the adsorbed layers and/or network effects [118]. Additional measurements are planned to 1$)$ resolve the low-pressure region $\left(<0.2 P / P_{0}\right)$ in order to study the initial adsorption of interfacial water and 2) to determine the porosity as in Fig. 2.14 but now using water as the probe molecule. 


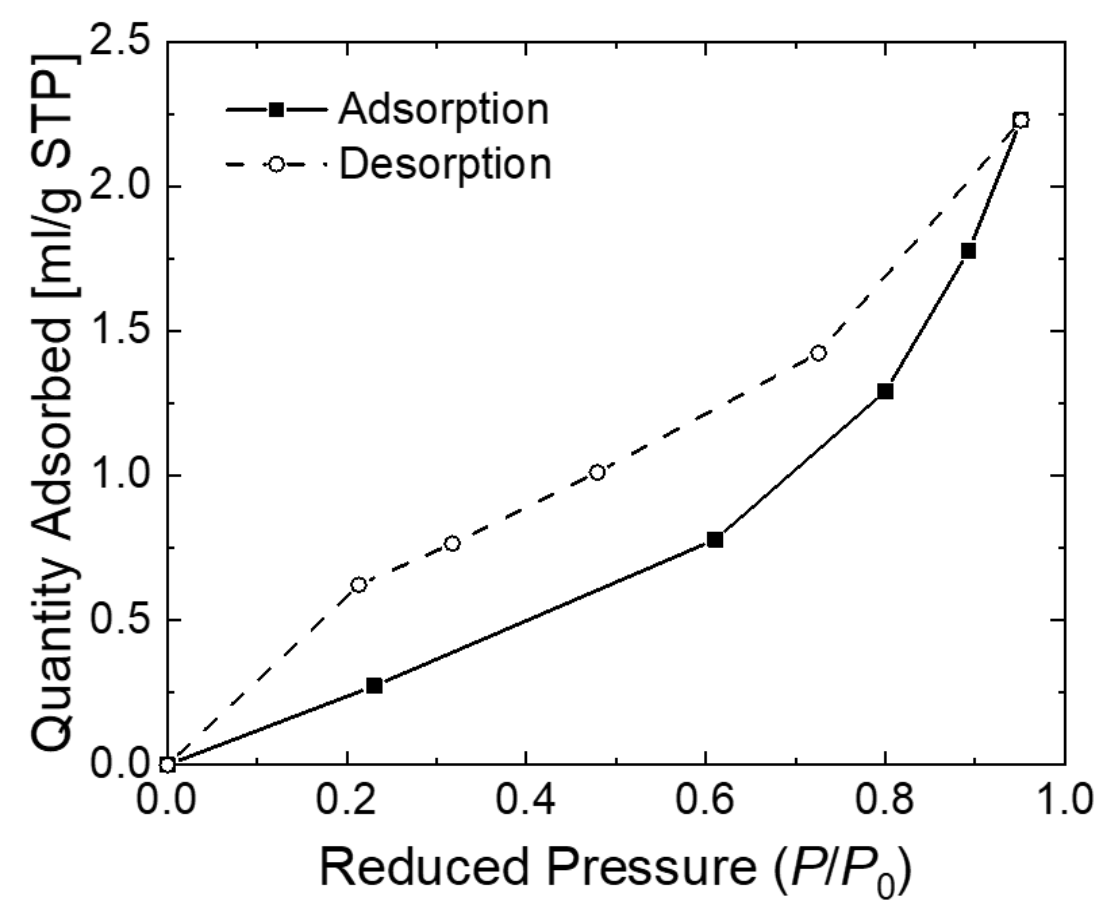

Figure 2.15. Water vapor adsorption and desorption isotherms measured at $298 \mathrm{~K}$.

We also performed atomic force microscopy (AFM) measurements to determine the surface roughness and estimate the surface area of the $\mathrm{CuO}$ coating in contact with hydration water. The high aspect ratio and density of the nanostructures made these measurements difficult because standard AFM probes cannot penetrate very far into the valleys between neighboring $\mathrm{CuO}$ blades. In addition, the AFM probe is not sensitive to the side of $\mathrm{CuO}$ blades facing the $\mathrm{Cu}$ substrate or blades oriented normal to the surface. Nevertheless, AFM scans were performed at the Center for Nanophase Materials Sciences (CNMS) at Oak Ridge National Laboratory using high-aspect ratio (>5:1) AFM probes (Asylum Research, AR5-NCH) and a state-of-the-art AFM instrument (Asylum Research, MFP-3D).

AFM scans were conducted in tapping-mode at a scanning rate of $0.1 \mathrm{~Hz}$ over a $10 \times 10 \mu \mathrm{m}^{2}$ area, and the results were averaged over ten different areas of a CuO-coated 
$\mathrm{Cu}$ foil. A representative AFM scan of a CuO sample is shown in Fig. 2.16. The rms $\left(\mathrm{R}_{\mathrm{q}}\right)$ roughness of the $\mathrm{CuO}$ surface is $\sim 739 \mathrm{~nm}$ with a standard deviation of $91 \mathrm{~nm}$, calculated using the following expression,

$$
R_{q}=\sqrt{\frac{1}{L} \int_{0}^{L}\left|Z^{2}(x)\right| d x}
$$

where $L$ is the length of the scan line and $Z(x)$ is the pixel height at position $x$ on the surface. The approximate surface area $\left(S_{m}\right)$ of the $\mathrm{CuO}$ coating in a $100 \mu \mathrm{m}^{2}$ scan area $\left(S_{a}\right)$ is $302 \mu \mathrm{m}^{2}$, which is calculated by a simple triangulation method [122] embedded in the AFM instrument's acquisition software. Using the mass of a single foil $(\sim 0.2 \mathrm{~g})$, the surface area measured by AFM equates to a specific surface area of $\sim 0.06 \mathrm{~m}^{2} / \mathrm{g}$, which is an order of magnitude smaller than that determined from a nitrogen isotherm $\left(\sim 0.6 \mathrm{~m}^{2} / \mathrm{g}\right)$. This discrepancy is due to the inability of the AFM probe to access a significant fraction $(90 \%)$ of the $\mathrm{CuO}$ pore space/surface area. It can be also be demonstrated by comparing the AFM image in Fig. 2.16 to an SEM image like that in Fig. 2.4f. For reference, the AFM scan of a $100 \mu \mathrm{m}^{2}$ area of a bare $\mathrm{Cu}$ foil (not shown) yields an rms roughness of $110 \pm 19 \mathrm{~nm}$ and surface area of $\sim 103.5 \pm 0.8 \mu \mathrm{m}^{2}$. Therefore, the roughness parameters $\left(r=S_{m} / S_{a}\right)$ for the $\mathrm{CuO}$ and $\mathrm{Cu}$ surfaces are $\sim 3.0$ and $\sim 1.0$, respectively. Our AFM results confirm that surface probes cannot access the entire surface area of these $\mathrm{CuO}$ nanostructured coatings and that vapor sorption measurements are preferred. 


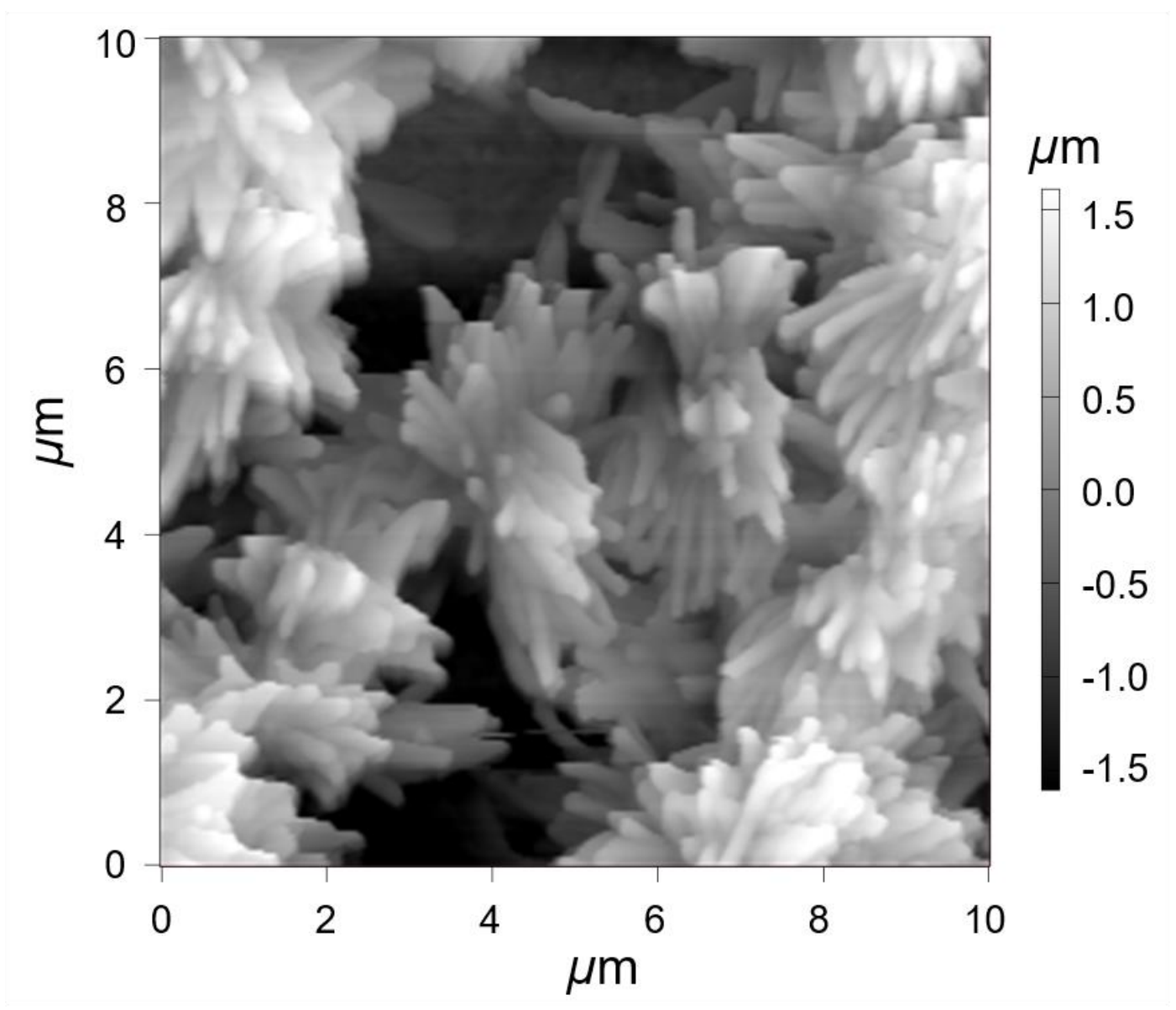

Figure 2.16. Topographical AFM image of a $10 \times 10 \mu \mathrm{m}^{2}$ region of a nanostructured $\mathrm{CuO}$ surface. 


\section{Chapter 3}

\section{Structure and Freezing/Melting Behavior of}

\section{Water Near Hydrophilic CuO Surfaces}

In this chapter, we assess the strength of the hydrophilic interaction between water and nanostructured-CuO surfaces by examining water's solid-liquid phase transition. Our approach utilizes incoherent elastic neutron scattered intensity measured as a function of sample temperature on a high-energy-resolution backscattering neutron spectrometer. These so-called elastic "fixed-window" scans (FWS) served as efficient preliminary measurements conducted prior to our more detailed quasielastic neutron scattering (QENS) experiments discussed in Chapter 4. Therefore, in Sec. 3.1 we introduce two backscattering spectrometers from which we collected FWS and QENS. The composition of our samples used for neutron scattering measurements is given in Sec. 3.2. The results of our FWS are presented in Sec. 3.3 where we report continuous freezing and melting of the water near $\mathrm{CuO}$-coated samples that is consistent with the formation of an amorphous solid phase. To test this hypothesis, we conducted a series of neutron diffraction measurements, with results presented in Sec. 3.4, to determine the structure of the solid interfacial water. The absence of Bragg peaks associated with crystalline ice supports our interpretation of the FWS wherein water freezes into an amorphous solid at low hydration ( $\leq 120 \mu 1 \mathrm{D}_{2} \mathrm{O}$ added). However, at sufficiently high hydration $(240 \mu \mathrm{l})$ Bragg peaks indexed to hexagonal ice appear around $260 \mathrm{~K}$ whose intensities grow with decreasing temperature. 


\subsection{Two Complementary Backscattering Neutron}

\section{Spectrometers}

Two state-of-the-art backscattering neutron spectrometers were used to study the freezing/melting behavior and dynamics of interfacial water near $\mathrm{CuO}$-coated and bare$\mathrm{Cu}$ foils: The High-Flux Backscattering Spectrometer (HFBS) at the NIST Center for Neutron Research [123] and the Backscattering Silicon Spectrometer (BaSiS) [124] at the ORNL Spallation Neutron Source. In general, backscattering spectrometers have an inverted geometry in which the final neutron energy is fixed via diffraction from an array of analyzer crystals. This setup is analogous to a triple-axis spectrometer with a fixed analyzer position. Furthermore, backscattering spectrometers are characterized by highenergy resolution $\Delta E_{\text {min }}$ in the $\mu \mathrm{eV}$ to sub- $\mu \mathrm{eV}$ range where the final neutron energy $E_{f}$ is determined by Bragg diffraction from an array of silicon (111) analyzer crystals at scattering angles approaching $2 \theta=180^{\circ}$ (backscattered) [123-124]. Given a fixed final energy, the incident neutron energy varies with its time-of-arrival, which is determined in fundamentally different ways for each spectrometer.

On $\mathrm{BaSiS}$, the incident neutron energy $E_{i}$ is determined by the time it takes for a neutron emitted from the surface of the moderator to reach the sample position, i.e., its time-of-flight (TOF). The incident energy is expressed as [125],

$$
E_{i}=\frac{\alpha L_{i}^{2}}{\left(t-t_{f}-t_{0}\right)^{2}}
$$


where $\alpha$ is a constant $\left(5.2276 \times 10^{-6}\right), L_{i}$ is the moderator-to-sample distance $(84 \mathrm{~m}), t$ is the total time-of-arrival measured by the detector, $t_{f}$ is the TOF of the neutron from the sample to the detector, and $t_{0}$ is the emission time of the neutron from the moderation (time measured in seconds). The uncertainty in the energy transfer $\delta E$ is given by the uncertainty in the incident and final energy terms, which add in quadrature [125],

$$
\delta E=\sqrt{\delta E_{P}^{2}+\delta E_{S}^{2}}
$$

where $\delta E_{P}$ and $\delta E_{S}$ are the contributions by the flight paths before and after the sample.

In contrast with BaSiS, on the HFBS the energy resolution is determined by the monochromator, which consists of an array of perfect silicon wafers oriented with their (111) planes normal to the incident neutron beam. The spread in wavelength of neutrons $\Delta \lambda$ that Bragg-diffract from the Si (111) planes of the monochromator is described by the following expression [123],

$$
\left(\frac{\Delta \lambda}{\lambda}\right)^{2}=\left(\frac{\Delta d}{d}\right)^{2}+\left(\frac{\Delta \theta}{\tan \theta}\right)^{2}
$$

where the last term vanishes for scattering angles approaching the backscattering condition $\left(\theta=90^{\circ}\right)$ and $\Delta d$ is the spread in the lattice spacing of the $\operatorname{Si}(111)$ planes. The Si wafers are mounted on an oscillating support structure (mechanical Doppler drive), which moves in a direction parallel to the average $\mathrm{Si}[111]$ direction such that the backscattering condition is always maintained [123]. The primary use of the Doppler drive is to change the incident neutron energy on the HFBS. With this technology, at any point in time, the energy of the neutron incident on the sample is shifted up or down 
(depending on the direction the Doppler drive moves) by diffraction from the moving Si(111) lattice [123].

The configurations of the HFBS and BaSiS used in the following experiments are summarized in Table 3.1. These spectrometers complement each other in that they access dynamics on similar time and length scales: hundreds-of-picoseconds to nanosecond in time, and angstrom-to-nanometer in space. BaSiS, with its larger dynamic range, can sample faster motions than HFBS, whereas the HFBS has a better energy resolution $(\sim 1$ $\mu \mathrm{eV}$ vs. $\sim 3.5 \mu \mathrm{eV}$ for $\mathrm{BaSiS})$ - a point to which we will return later.

One of the primary data-acquisition modes of the HFBS is a scan of elastically scattered neutron intensity versus temperature, i.e., a "fixed-window scans (FWS)," as recorded in each detector (each wave vector transfer $Q$ ). It is obtained by halting the monochromator and recording the resolution-limited elastic intensity. A FWS can also be recorded as a function of other physical variables like time or pressure. The strength of this operational mode is that all the neutrons incident on the sample have nominally the same energy, which allows for faster measurements of the elastic intensity as opposed to when the Doppler drive is in motion.

\begin{tabular}{cccc}
\multicolumn{1}{c}{} & $\begin{array}{c}Q \text {-range }\left[\AA^{-1}\right] \\
(d \text {-spacing }[\AA])\end{array}$ & $\begin{array}{c}\text { Dynamic range }[\mu \mathrm{eV}] \\
(\text { Approx. timescale }[\mathrm{ps}])\end{array}$ & $\begin{array}{c}\text { Energy resolution }[\mu \mathrm{eV}] \\
\text { (Approx. timescale }[\mathrm{ps}])\end{array}$ \\
\cline { 2 - 4 } HFBS & $0.25-1.75$ & \pm 17 & 1.0 \\
& $(\sim 3.59-25.12)$ & $(>240)$ & $(4100)$ \\
\cline { 2 - 4 } BASIS & $0.20-1.90$ & \pm 120 & 3.5 \\
& $(\sim 3.31-31.4)$ & $(>30)$ & $(1200)$
\end{tabular}

Table 3.1. Approximate wave vector transfer $(Q)$ ranges, energy-transfer (dynamic) ranges, and energy resolutions of the HFBS and BaSiS instruments used in QENS experiments. The conversion $d \sim 2 \pi / Q$ is used to estimate the accessible length scales ( $d$-spacings); time scales are estimated by the conversion $t \sim E / h$ where $h$ is Planck's 
constant. Note that the energy ranges and resolutions of both the HFBS and BaSiS are not limited to the specific configurations listed above.

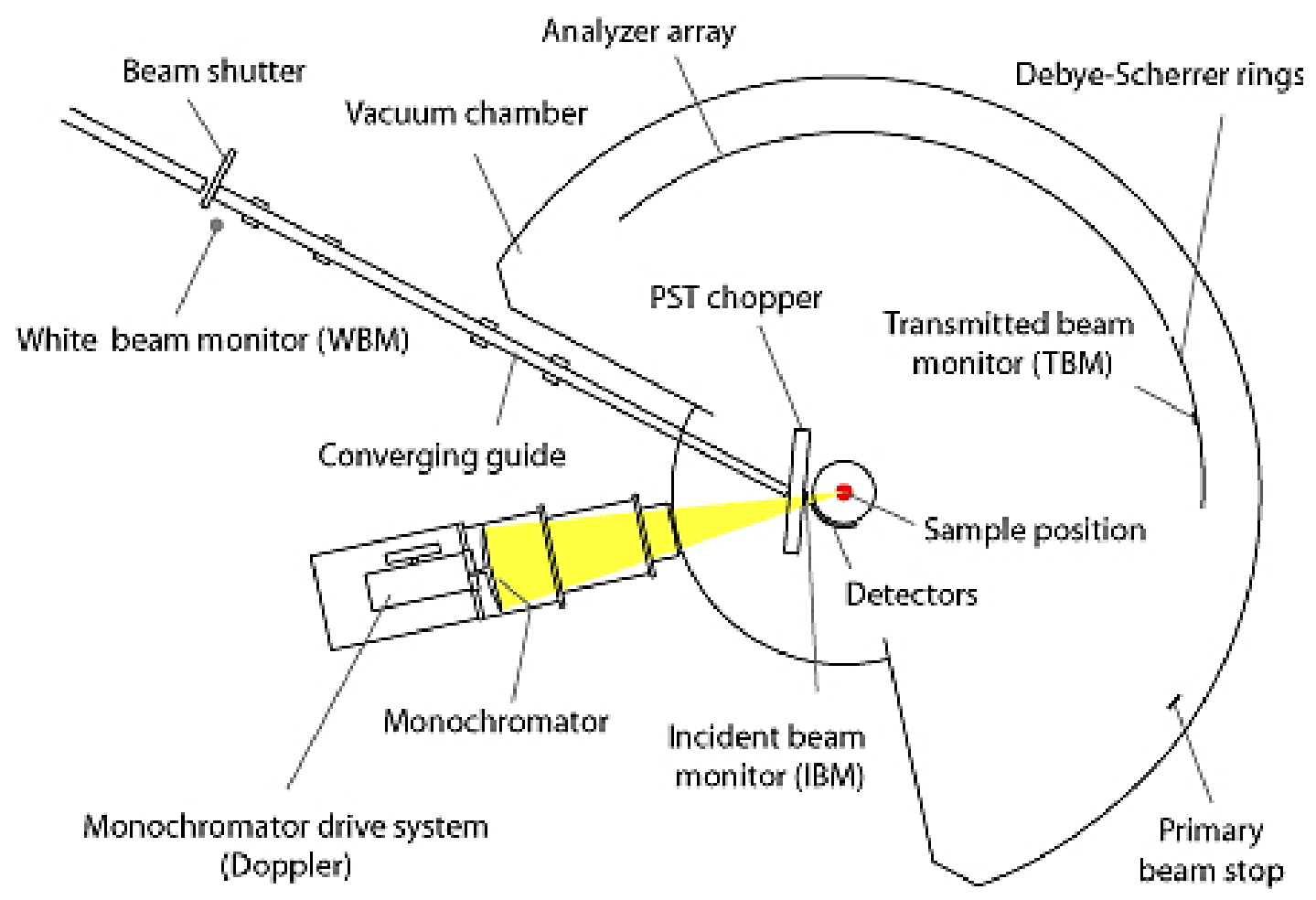

Figure 3.1. Schematic of the HFBS showing the major components of the instrument (reproduced from ncnr.nist.gov (accessed 2019)).

\subsection{Preparation of Samples for Neutron Scattering}

The samples used in the following neutron scattering experiments were prepared with identical composition and geometry to facilitate comparison of results between different spectrometers. Our samples consist of $100 \mathrm{CuO}$-coated copper foil disks (5-cm-diameter), each of thickness $12.7 \mu \mathrm{m}$, stacked inside of a cylindrical aluminum cell (5-cm-dia., 2cm-height) under helium atmosphere and sealed with an indium O-ring (see Fig. 3.2). In some experiments, a sample of untreated (bare) copper foils was prepared for background subtraction or comparison purposes. Prior to sealing a sample, and unless otherwise 
specified, the foils are heated in air at a moderately low temperature of $\sim 328 \mathrm{~K}$ for $24-48$ hours to drive off excess water. Then, the hydration level of each sample is established by loading a fixed volume of water $\left(\mathrm{H}_{2} \mathrm{O}\right.$ for incoherent scattering or $\mathrm{D}_{2} \mathrm{O}$ for diffraction) in the bottom of the sample cell immediately prior to sealing. After sealing the sample, it is heated again at $\sim 328 \mathrm{~K}$ for a couple of hours to evaporate the water and create a humid atmosphere inside of the cell. A list of the samples and sample cell used in our neutron scattering experiments is given in Appendix A.

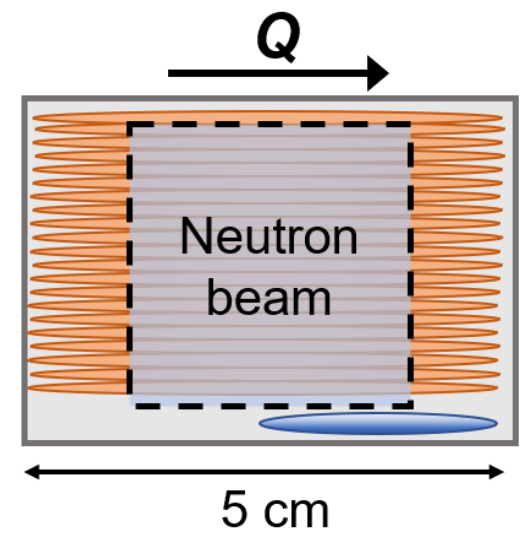

Figure 3.2. Sketch of the sample cell used for neutron scattering experiments. A water droplet $\left(\mathrm{H}_{2} \mathrm{O}\right.$ or $\left.\mathrm{D}_{2} \mathrm{O}\right)$ of known volume placed at the bottom of the cell, outside of the neutron scattering volume, controls the hydration level. The scattering vector $\boldsymbol{Q}$ lies in the plane of the copper foils. The incident neutron beam cross section has dimensions $\sim 30 \mathrm{x}$ $30 \mathrm{~mm}^{2}$. Not drawn to scale.

\subsection{Elastic "Fixed-Window" Scans Measured on the}

\section{HFBS}

In addition to the wetting behavior investigated with water contact angle measurements and environmental-SEM, both discussed in Sec. 2.2, the strength of the hydrophilicity of $\mathrm{CuO}$ nanostructures can be assessed by studying their effect on water's freezing transition. Differential scanning calorimetry (DSC) is a convenient and frequently 
employed technique used to investigate freezing and melting transitions of water confined in porous media (see, e.g., Ref. [126]). We have tried to apply the DSC technique to our system, but the specific surface area of a CuO-coated $\mathrm{Cu}$ foil $\left(\sim 0.6 \mathrm{~m}^{2} / \mathrm{g}\right)$ is extremely small. Our DSC samples contain an amount of water (few $\mu \mathrm{g})$ that is two to three orders of magnitude less than the quantity typically used (few mg) in measurements with commercial DSC instruments. For this reason, we have been unable to perform reliable DSC scans to investigate the freezing/melting behavior of our samples.

The size of the neutron beam of the HFBS $\left(\sim 3 \times 3 \mathrm{~cm}^{2}\right.$ cross-section) accommodates much larger samples than those used in DSC measurements. The large number (100) and diameter $(5 \mathrm{~cm})$ of the $\mathrm{CuO}$-coated copper foils increase the total surface area of the $\mathrm{CuO}$ coating and therefore enhance the scattering from interfacial water. Due to the large incoherent cross section of hydrogen, neutrons are highly sensitive to the thin layer of interfacial water, and FWSs provide an attractive probe of the phase transitions of water. Specifically, a FWS is sensitive to water molecules which are immobile or move on a time scales slower than $\sim 4 \mathrm{~ns}$ as determined by the energy resolution of the HFBS. As the sample temperature is lowered, an increase in the elastic intensity measured on HFBS provides a measure of the amount of water which becomes immobile or "frozen" on a nanosecond time scale. In contrast, DSC scans integrate molecular motions over a wider range of timescales.

Figure 3.3 shows the FWSs of our three samples: $\mathrm{CuO}$-coated $\mathrm{Cu}$ foils hydrated with $60 \mu \mathrm{l}$ of $\mathrm{H}_{2} \mathrm{O}$ (termed "wet"); a similar sample with $10 \mu \mathrm{l}$ of $\mathrm{H}_{2} \mathrm{O}$ (termed "dry"); and, for comparison, a sample of bare $\mathrm{Cu}$ foils with $60 \mu \mathrm{l}$ of $\mathrm{H}_{2} \mathrm{O}$. All three samples were cooled and heated at a slow temperature ramp rate of $0.08 \mathrm{~K} / \mathrm{min}$ to establish quasi- 
equilibrium of the system, and each data point of Fig. $\mathbf{3 . 3}$ was integrated over a counting time of 5 minutes. Scans began above the freezing point of bulk water at $280 \mathrm{~K}$, cooled to $200 \mathrm{~K}$, and then immediately heated back to $280 \mathrm{~K}$ over a time span of about 42 hours during which data were recorded. The elastic intensity was summed over all 16 detectors spanning a wave vector transfer $(Q)$ range of $0.25 \AA^{-1}<Q<1.75 \AA^{-1}$ and then normalized to the incident white-beam monitor located upstream of the sample position (see Fig. 3.1).

As shown in Fig. 3.3a, the elastic intensity of the wet CuO-coated sample gradually increased when slow cooled from $280 \mathrm{~K}$ to $200 \mathrm{~K}$. On heating at the same rate from $200 \mathrm{~K}$ to $280 \mathrm{~K}$, hysteresis was observed with the intensity gradually decreasing toward its initial value. A similar trend was observed in the dry sample, although with reduced hysteresis. The total change in the intensity between the highest and lowest temperatures is less than for the wet sample. We checked the reproducibility of the FWS in a second cooling cycle of the dry CuO-coated sample. A slight decrease in the slope of the high-temperature data was observed and attributed to an increase in the low- $Q$ intensity $\left(Q \leq 0.36 \AA^{-1}\right)$. Due to time constraints, a second cooling scan on the wet CuOcoated sample was not completed; however, data in the temperature range $250-280 \mathrm{~K}$ was in excellent agreement with the first scan.

We have interpreted the gradual increase (decrease) in the elastic intensity as indicating a continuous freezing (melting) transition of the water in the samples. This freezing behavior differs that of water near the bare $\mathrm{Cu}$ surface where the abrupt changes in the intensity are observed on cooling at $\sim 266 \mathrm{~K}$ and, on heating, at the bulk melting point of water, $273 \mathrm{~K}$ (Fig. 3.3c). 
By recalling that the change in the elastic intensity in a temperature interval is proportional to the amount of water that immobilizes, we note that the factor of $\sim 2$ difference in the intensity between the wet and dry CuO-coated samples over the temperature range $200-280 \mathrm{~K}$ does not reflect their 6:1 hydration ratio. Assuming that all of the initial water droplet is adsorbed onto the CuO-coated foils, there are several possible reasons for this discrepancy: (1) irreproducibility in the amount of residual water on the foils after annealing the samples [127], (2) variations in the CuO coating's surface area and/or hydrophilicity, and (3) a difference in the position of the Doppler drive of the HFBS between elastic scans $[\mathbf{1 2 3}, \mathbf{1 2 8}]$.

As seen in the elastic scans of the dry CuO-coated sample in Fig. 3.3b, we find that, on cooling, the elastic intensity obeys a linear temperature dependence from $280 \mathrm{~K}$ down to $\sim 237 \mathrm{~K}$. Similarly, the intensity of the wet $\mathrm{CuO}$ sample (Fig. 3.3a) has an initial linear temperature dependence with virtually the same slope as that of the dry sample. As will be discussed further below, this initial linear term occurring in the freezing of both the wet and dry samples suggests that they share a common population of water confined in a similar environment [129]. The initial freezing behavior of the wet sample, however, ends at $\sim 258 \mathrm{~K}$ followed by an increase in the slope that extends down to $\sim 237 \mathrm{~K}$ - the same temperature at which the linear dependence of the intensity of the dry sample ends. Based on these observations, we suggest that both linear terms contribute to the intensity of the wet sample in the temperature range $237-258 \mathrm{~K}$. The presence of a steeper linear term in the elastic intensity scan of this sample provides evidence of a second water population occupying a distinct nanoscale environment. As this second population freezes more rapidly with temperature than the first, we suggest this water is located 
either (1) further from the $\mathrm{CuO}$ surfaces or (2) in a less dense region of the nanostructures (e.g., further up on a $\mathrm{CuO}$ blade). The first possibility (1) is based on investigations of porous silicas where it is inferred bulk-like water freezes further from the pore walls atop a layer of bound water [68]. The latter possibility (2), however, seems more likely to be the case in the $\mathrm{CuO}$ system as it is supported by our in-situ condensation experiments from Sec. 2.2, which will be discussed further in Sec. 4.2.6. 


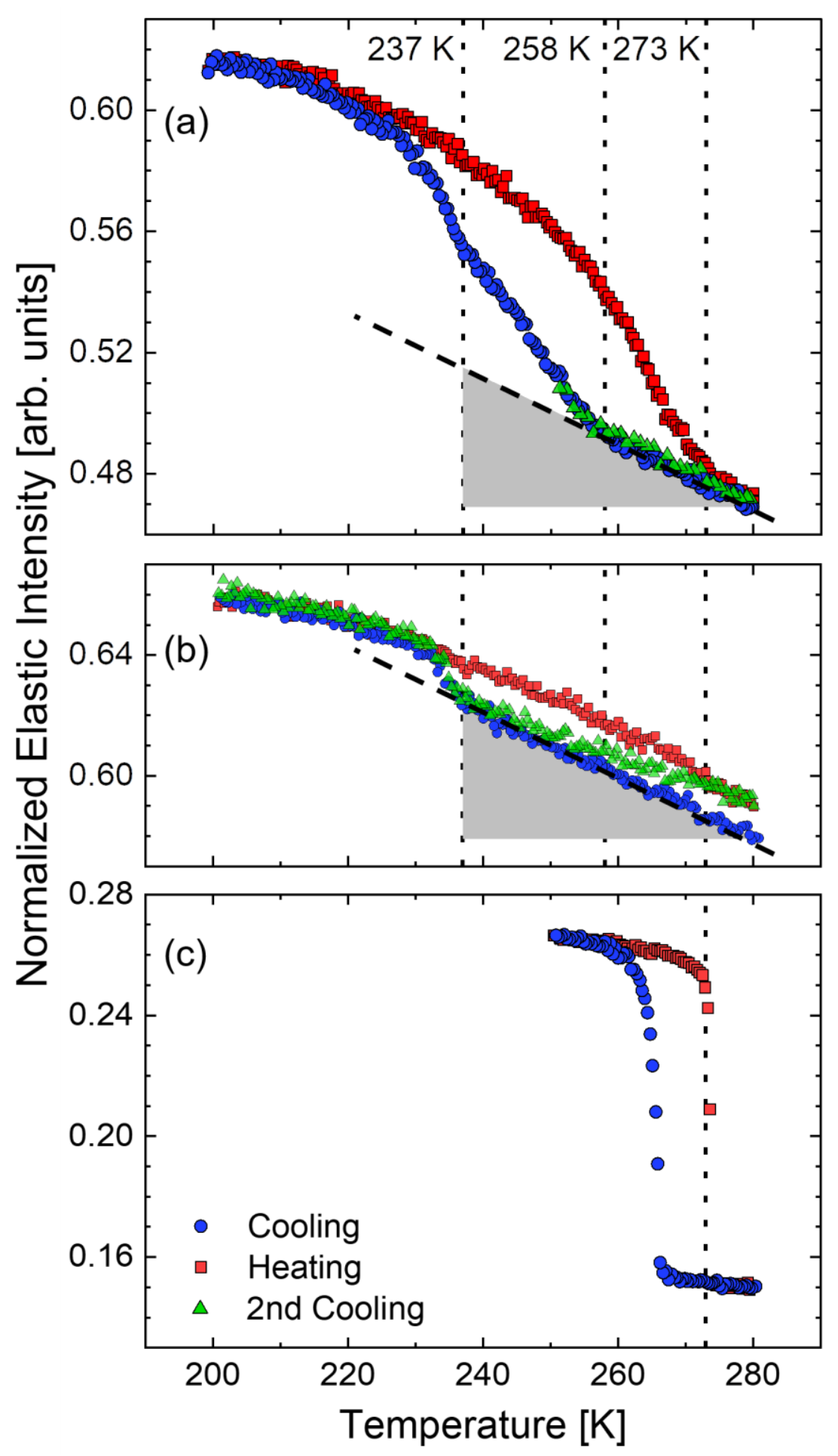

Figure 3.3. Elastic scans measured on the HFBS vs. sample temperature of three samples: (a) CuO-coated $\mathrm{Cu}$ foils with $60 \mu 1$ of $\mathrm{H}_{2} \mathrm{O}$; (b) a similar sample with $10 \mu 1$ of $\mathrm{H}_{2} \mathrm{O}$; and (c) bare $\mathrm{Cu}$ with $60 \mu \mathrm{l}$ of $\mathrm{H}_{2} \mathrm{O}$. Intensity was summed over all 16 detectors covering a wave vector transfer $(Q)$-range of $0.25 \AA^{-1}<Q<1.75 \AA^{-1}$ and normalized to the incident beam monitor (see Fig. 3.1). Cooling (blue and green) and heating (red) scans were performed at a slow temperature ramp rate of $0.8 \mathrm{~K} / \mathrm{min}$ between $200 \mathrm{~K}$ and $280 \mathrm{~K}$. 
The continuous freezing behavior observed for water in proximity to the $\mathrm{CuO}$ coated surfaces differs from that which we have found for water near other interfaces. For example, the water associated with an anionic bilayer lipid membrane also exhibited a continuous freezing behavior, but its elastic scan on cooling began with a step-like feature, somewhat broader than we have found for our bare-Cu sample in Fig. 3.3c and whose magnitude increased with the water content of the sample (see Fig. 3 in Ref. [130]). This behavior suggested identifying it with the freezing of bulk-like water as was subsequently verified by measurement of its diffusivity [129]. Such a step-like feature is missing in the case of water near the $\mathrm{CuO}$ nanostructures, suggesting the absence of bulklike water.

There are reports of continuous freezing of interfacial water in other porous materials. Using neutron elastic scans with $1 \mu \mathrm{eV}$ resolution, Liu et al. [131] observed a gradual increase in elastic intensity from $300 \mathrm{~K}$ down to $150 \mathrm{~K}$ for water confined in mesoporous silica (MCM-41 and MCM-48) having pore diameters in the 14-22 $\AA$ range. In a more complex mesoscopic environment, Mamontov et al. [127] found qualitatively similar freezing behavior of water adsorbed in ultra-microporous carbon. As in our samples, they observed that the elastic intensity (intensity recorded over an energy transfer range of $\pm 3.4 \mu \mathrm{eV}$ around the elastic peak) increased linearly with temperature as the sample was cooled below room temperature at relatively low humidity (hydration) levels [127]. Unlike our samples, however, the slope of their initial linear term increased with water content, suggesting that a greater amount of water occupied a similar environment. At the highest relative humidity, the temperature dependence of the elastic 
intensity was initially concave upward, perhaps indicating the freezing of bulk-like water within the larger pores.

The elastic intensity measured in Fig. 3.3 includes contributions from both the $\mathrm{H}$ and $\mathrm{Cu}$ atoms of the sample; however, the signal is dominated by the incoherent scattering from the $\mathrm{H}$ atoms of the water in proximity to the $\mathrm{CuO}$ coating. Assuming that the contribution from the $\mathrm{Cu}$ atoms can be neglected, we can determine the $Q$ dependence of the elastic intensity $I(Q)$ in the cooling cycle by using a Debye-Waller model as in Eq. (3.4) [127-128],

$$
I(Q)=I_{0}(Q) e^{-Q^{2}\left\langle u^{2}\right\rangle / 3}
$$

where $\left\langle u^{2}\right\rangle$ is the mean-squared displacement (MSD) of $\mathrm{H}$ atoms and $I_{0}(Q)$ is the measured elastic intensity at the lowest temperature of $200 \mathrm{~K}$.

Shown in Fig. 3.4 are the MSDs of the wet and dry samples on cooling from 280 $\mathrm{K}$ to $200 \mathrm{~K}$ at $0.08 \mathrm{~K} / \mathrm{min}$. At $280 \mathrm{~K}$, the MSD of the wet sample is at least a factor of two higher than that of the dry sample, and the value of $0.55 \AA^{2}$ at $240 \mathrm{~K}$ is consistent with the $\sim 0.6 \AA^{2}$ measured independently by Kiwilsza et al. [97] for water in SBA-15 and by Zanotti et al. [80] for water in Vycor. The latter group interpreted the inflection in the MSD at $240 \mathrm{~K}$ as a phase transition from a high-density to a low-density liquid state. We consider this possibility in Sec. 4.2.6 for our Cu-coated samples. As shown in Fig. 3.4, the MSD of our wet sample decreases roughly linearly on cooling in two temperatures intervals: 280-258 K and, with a larger slope, in the range 258-237 K. At lower temperatures, the MSD levels-off, indicating the immobilization of the water molecules on a nanosecond time scale. 


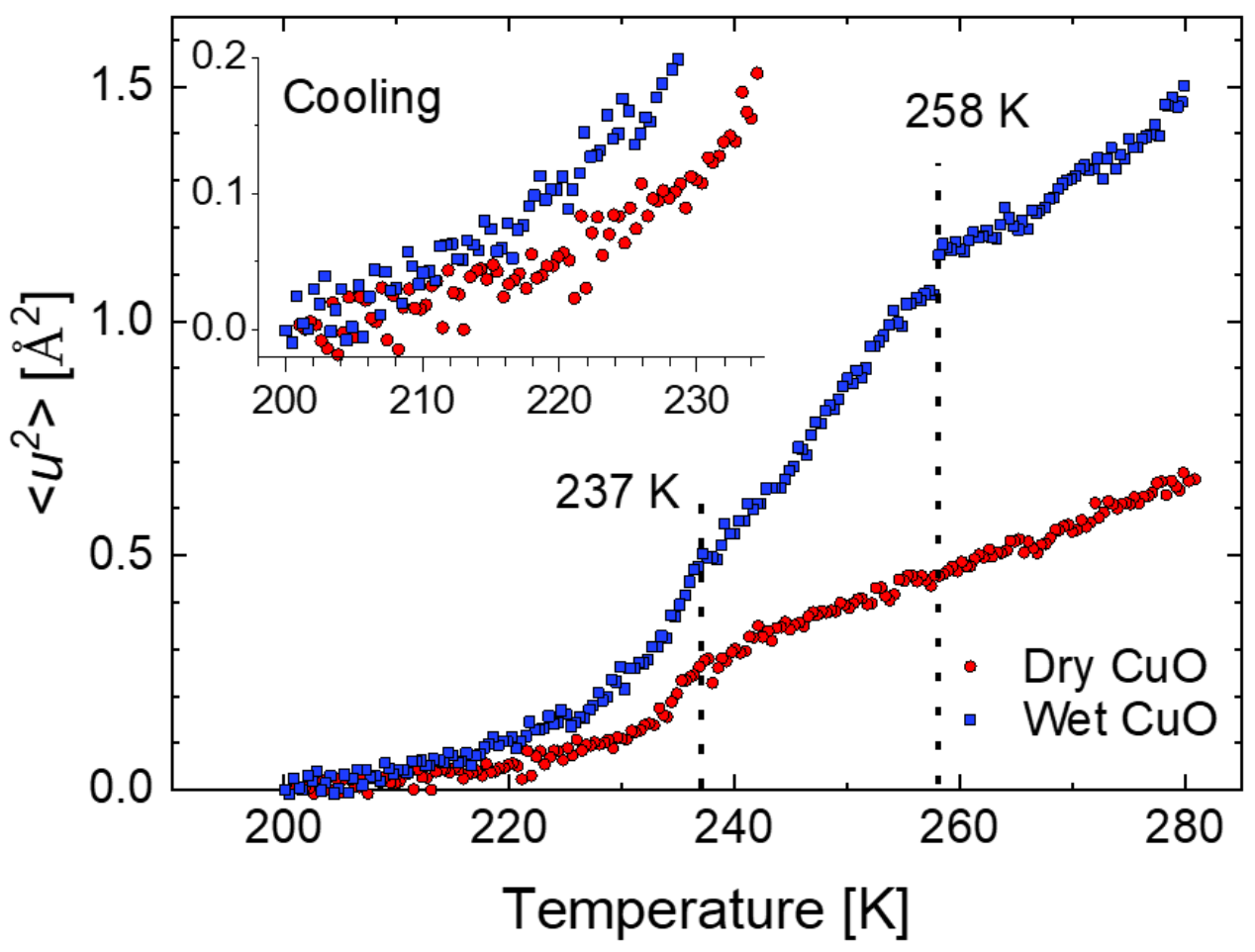

Figure 3.4. Mean-squared displacement (MSD, $\left.\left\langle u^{2}\right\rangle\right)$ plotted vs. temperature on cooling at a constant ramp rate of $0.08 \mathrm{~K} / \mathrm{min}$ for the wet (blue) and dry (red) CuO-coated samples. Data were summed over the $Q$ range $0.36-1.75 \AA^{-1}$. Vertical dotted lines and arrows are drawn at inflection points in the data. Inset shows the MSDs over the low temperature range.

To quantify the hydration level in our samples, we estimated the number of immobilized water molecules from the increase in elastic intensity on cooling from $280 \mathrm{~K}$ to $200 \mathrm{~K}$. This number was determined by calibration against a standard sample of alkane $\left(\mathrm{C}_{32} \mathrm{H}_{66}\right)$ films containing a known number of $\mathrm{H}$ atoms that freeze on a silicon substrate [129]. A correction was applied for the larger incoherent cross section of our predominantly copper substrates relative to silicon. Results show a total volume of $\sim 80 \mu \mathrm{l}$ and $\sim 40 \mu \mathrm{l}$ of water that becomes immobile between $280 \mathrm{~K}$ and $200 \mathrm{~K}$ for the wet and dry samples, respectively. For both samples, the calculated water volumes exceed those in the initial droplet, suggesting the presence of residual water after the samples were annealed. Evidence of strongly bound water has also been found on other oxide surfaces 
at high temperatures [132]. Assuming the water wets to the $\mathrm{CuO}$ coating in the form of a thin film with a uniform thickness over both sides of a foil, we estimate an upper bound to the water film thickness of the wet and dry samples to be $\sim 200 \mathrm{~nm}$ and $\sim 100 \mathrm{~nm}$, respectively. Although the uncertainty in these estimates is large, we believe it is reasonable to conclude that the water film thickness is less than the typical height of a CuO blade $(\sim 2 \mu \mathrm{m})$. 


\subsection{Neutron Diffraction}

Motivated by the results of our elastic FWS presented in the previous section, we performed a series of neutron diffraction measurements at the University of Missouri Research Reactor (MURR) on similarly fabricated samples to determine if the water near the $\mathrm{CuO}$ coating forms a crystalline or amorphous solid as a function of both temperature and hydration level. Samples were hydrated with $\mathrm{D}_{2} \mathrm{O}$ instead of $\mathrm{H}_{2} \mathrm{O}$ (to enhance coherent scattering) and were cooled from $300 \mathrm{~K}$ to $200 \mathrm{~K}$ at $\sim 1 \mathrm{~K} / \mathrm{min}$. Diffraction scans were taken at fixed temperatures in $5 \mathrm{~K}$-increments on cooling to $200 \mathrm{~K}$. At each temperature, a diffraction scan was recorded over a counting time of approximately three to twelve hours, depending on the chosen scattering range. By these stepwise scans, we searched for Bragg peaks that could be indexed to crystalline ice and which could provide insight into the continuous freezing/melting of water discussed in the previous section.

We used the two-axis neutron powder diffractometer located at D-port at MURR (Fig. 3.5) with a monochromatic beam of $\lambda=1.485 \AA$ neutrons and a detection system consisting of a vertical stack of five linear position-sensitive $\mathrm{He}^{3}$ detectors [135]. The 24inch active length of the 1-inch-diameter detectors subtends a scattering $\operatorname{arc}$ of $2 \theta \sim 20^{\circ}$. The detector bank is mounted on a moveable arm that covers a scattering range of $0-105^{\circ}$ in a series of five adjustable arm positions. At each arm position, six million counts of the monitor (counting time of $\sim 3$ hours), located before the sample, allows normalization of the diffraction patterns. In most cases, a single arm position is used that spans the range $19.45^{\circ}<2 \theta<39.40^{\circ}$ (equivalently, $1.43 \AA^{-1}<Q<2.85 \AA^{-1}$ where $Q=4 \pi \sin (2 \theta / 2) / \lambda$ ) because the most intense Bragg peaks for bulk hexagonal or cubic ice can be found in this 
range [136]. Full-range (complete) diffraction scans were also taken at least once during each cooling cycle to determine if other forms of ice are present or if structural changes are evident at higher scattering angles.

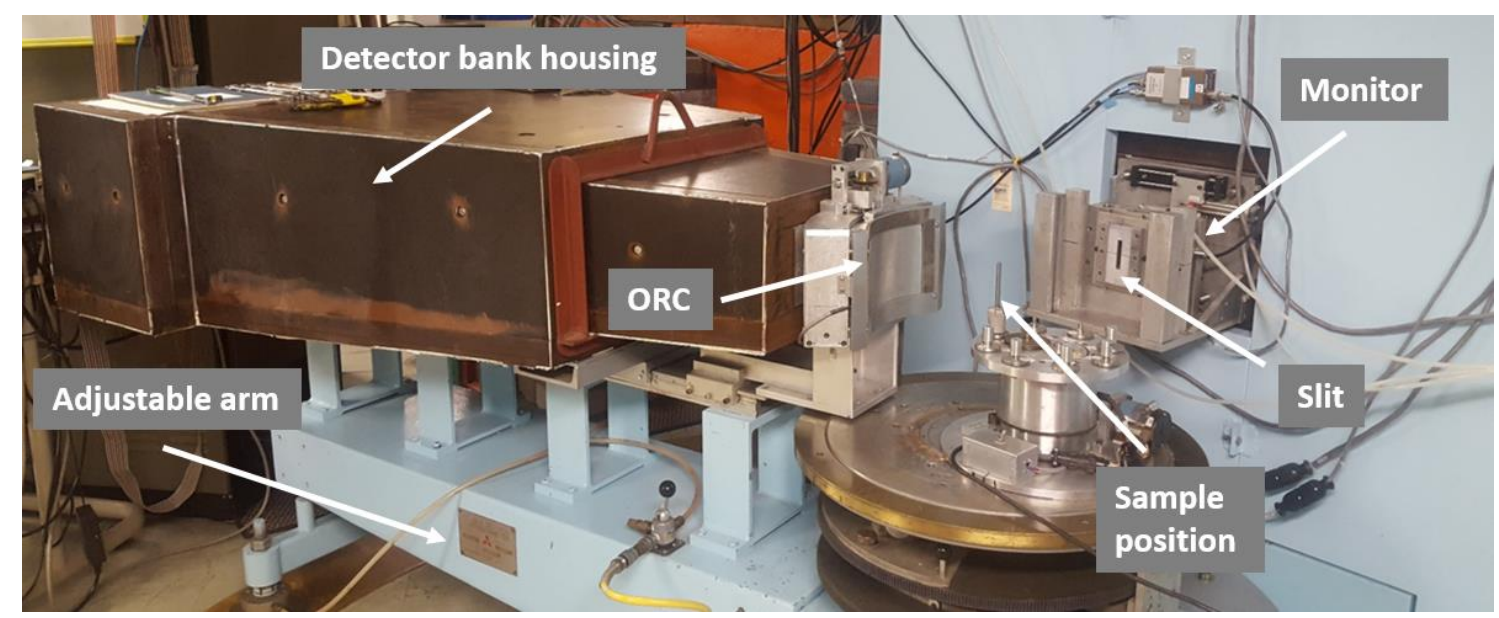

Figure 3.5. Photo of the two-axis powder neutron diffractometer located at D-port at MURR with several major components labeled (e.g., oscillating radial collimator ORC). Courtesy of Z. N. Buck [137].

A complete room-temperature $(\sim 300 \mathrm{~K})$ diffraction pattern of a non-hydrated $(0$ $\mu \mathrm{l}$ of $\mathrm{D}_{2} \mathrm{O}$ ) CuO-coated sample is shown in Fig. 3.6. Bragg peak positions, intensities, and widths were determined by Gaussian fits of the form,

$$
y=y_{0}+\frac{A}{\left(w \sqrt{\frac{\pi}{2}}\right)} \exp \left[-\frac{1}{2}\left(\frac{x-x_{c}}{w}\right)^{2}\right]
$$

where $y_{0}$ is a free parameter and $x_{c}, w$, and $A$ are the peak position, width, and intensity, respectively. The observed peak positions were then compared with the calculated positions (assuming a neutron wavelength of $\lambda=1.485 \AA$ ) for bulk copper $(\mathrm{Cu})$, cuprous oxide $\left(\mathrm{Cu}_{2} \mathrm{O}\right)$, and cupric oxide $(\mathrm{CuO})$ phases. From this analysis, we find good agreement between the calculated and observed peak positions as shown in Table 3.2. 


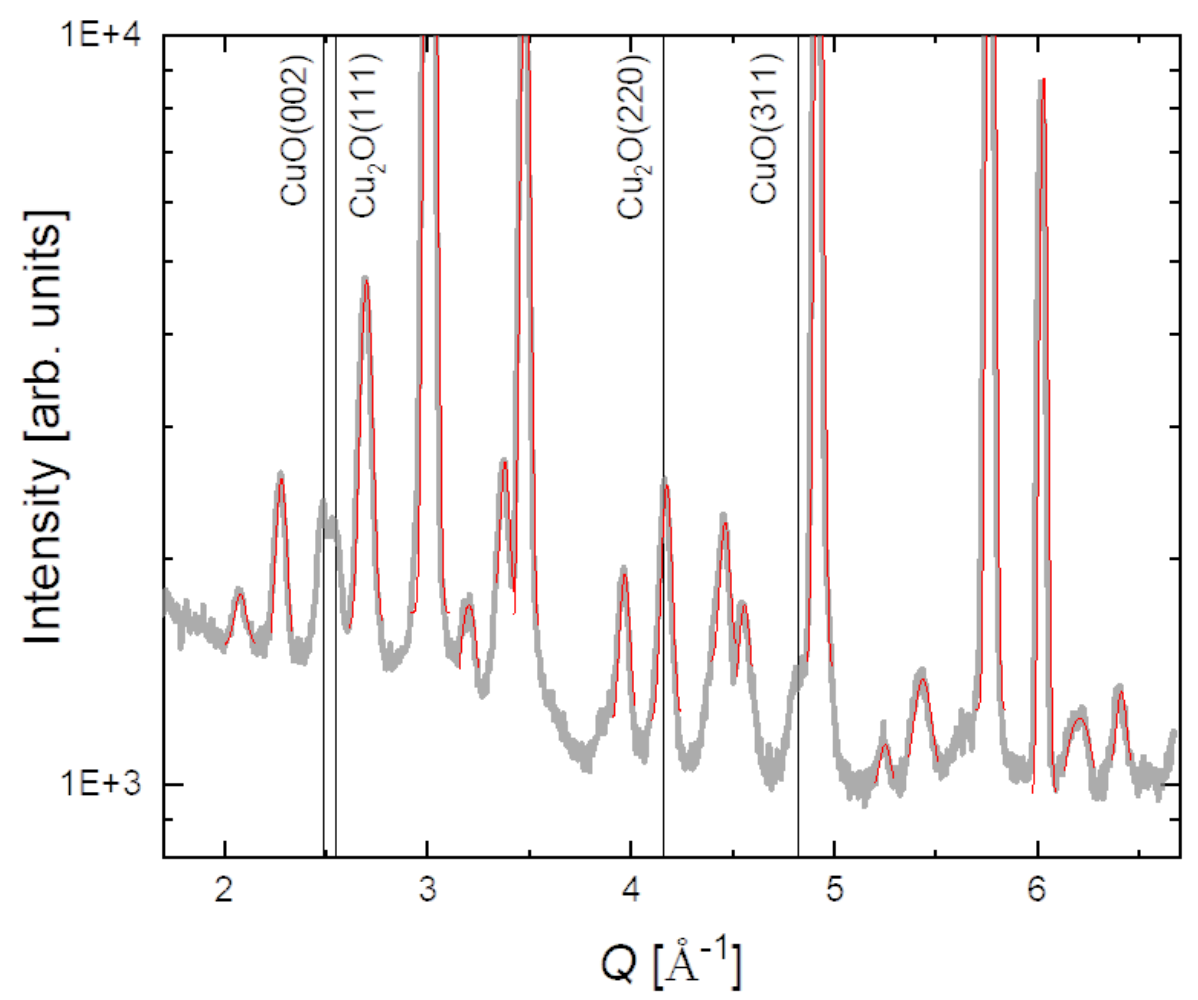

Figure 3.6. Semi-log plot of the diffraction intensity for a CuO-coated sample at $\sim 300 \mathrm{~K}$, showing Gaussian fits (red curves fit to Eqn. (3.5)) for most of the Bragg peaks.

Overlapping peaks are also shown with their approximate positions indicated by vertical lines and indexed as in Table 3.2.

\begin{tabular}{|c|c|c|c|c|c|c|c|c|}
\hline \multicolumn{3}{|c|}{$\mathrm{Cu}$} & \multicolumn{3}{|c|}{$\mathrm{Cu}_{2} \mathrm{O}$} & \multicolumn{3}{|c|}{$\mathrm{CuO}$} \\
\hline hkl & calc. $^{\text {a }} Q$ & obs. $Q$ & hkl & calc. ${ }^{\mathrm{b}} Q$ & obs. $Q$ & hkl & calc. $^{\text {c }} Q$ & obs. $Q$ \\
\hline 111 & 3.010 & 3.015 & 110 & 2.081 & $2.080(1)$ & 110 & 2.285 & 2.281 \\
\hline 200 & 3.476 & 3.482 & 111 & 2.549 & overlap & 002 & 2.485 & overlap \\
\hline 220 & 4.916 & 4.925 & 220 & 4.162 & buried? & 111 & 2.706 & 2.700 \\
\hline 311 & 5.765 & 5.771 & $\begin{array}{c}330 / \\
411\end{array}$ & 6.244 & $6.205(1)$ & $11-2$ & 3.205 & $3.203(1)$ \\
\hline 222 & 6.021 & 6.029 & 331 & 6.415 & $6.411(1)$ & $\begin{array}{c}20-2 \\
202 \\
11-3 \\
310 \\
113 \\
311 \\
12-3 \\
400\end{array}$ & $\begin{array}{l}3.367 \\
3.977 \\
4.175 \\
4.475 \\
4.560 \\
4.822 \\
5.248 \\
5.441\end{array}$ & $\begin{array}{c}3.380 \\
3.970 \\
4.176 \\
4.463 \\
4.558 \\
\text { overlap } \\
5.249(2) \\
5.435\end{array}$ \\
\hline
\end{tabular}

Table 3.2. Calculated and observed Bragg peak positions of $\mathrm{Cu}, \mathrm{Cu}_{2} \mathrm{O}$, and $\mathrm{CuO}$ phases in the diffraction pattern of a non-hydrated CuO-coated sample in Fig. 3.6. The Bragg peaks are labeled by their Miller indices (hkl) and their positions are given by the wave vector transfer $Q$ (in units of $\AA^{-1}$ ). ${ }^{\text {a }}$ from Ref. [138]. ${ }^{b}$ from Ref. [139]. ${ }^{\text {c }}$ from Ref. [140]. 
Based on the observed reflections in Fig. 3.6, the substrate consists of monoclinic $\mathrm{CuO}$, cubic $\mathrm{Cu}_{2} \mathrm{O}$, and fcc $\mathrm{Cu}$ in agreement with our SAED analysis (Fig. 2.7b). The weakness of the $\mathrm{Cu}_{2} \mathrm{O}$ and $\mathrm{CuO}$ peak intensities compared to those from $\mathrm{Cu}$ is consistent with the thickness of the oxide layers identified by electron microscopy in Fig. 2.7a compared to the that of the $\mathrm{Cu}$ substrate, which is about a factor of five thicker. In Fig. 3.6, there is a broad feature near $Q=2.5 \AA^{-1}$ that appears to be an overlap of two adjacent peaks, which, based on the reflections listed in Table 3.2, are most likely the $\mathrm{CuO}(002)$ and $\mathrm{Cu}_{2} \mathrm{O}$ (111) reflections. Similarly, the shoulder on the low- $Q$ side of the $\mathrm{Cu}$ (220) peak is possibly the $\mathrm{CuO}(311)$ reflection.

Due to the weak intensity expected for Bragg peaks from crystalline ice, we began our hydration-dependent measurements with samples of untreated and $\mathrm{CuO}$-coated $\mathrm{Cu}$ foils containing $120 \mu \mathrm{l}$ of $\mathrm{D}_{2} \mathrm{O}$ - twice the hydration level as the "wet" sample in our FWS in Fig. 3.3a. As shown in Fig. 3.7a, the pattern for the bare-Cu sample at $\sim 240 \mathrm{~K}$ contains three prominent Bragg peaks that could be indexed to hexagonal $\mathrm{D}_{2} \mathrm{O}$ ice [136]. These peaks initially appeared on cooling at $\sim 272 \mathrm{~K}$ or about $5 \mathrm{~K}$ below the freezing point of bulk $\mathrm{D}_{2} \mathrm{O}$. The approximately 5 degrees of supercooling is consistent with the $\sim 7$ degrees of supercooling observed in the FWS of the bare-Cu sample in Fig. 3.3. For the CuO-coated sample containing the same amount of water, no Bragg peaks were identified that could indicate the growth of crystalline ice (Fig. 3.7c). Moreover, the pattern is virtually identical to that of the sample containing no $\mathrm{D}_{2} \mathrm{O}$ (Fig. 3.7d) and remained unchanged down to $180 \mathrm{~K}$. In particular, no new Bragg peaks appeared on cooling that could indicate other forms of crystalline ice (see Fig. C.1). The maximum uncertainty in 
the temperature measurement is $\sim 2 \mathrm{~K}$, based on a temperature gradient between sensors at the top and bottom of the $2.6 \mathrm{~cm}$ tall sample cell (see Fig. A.2).

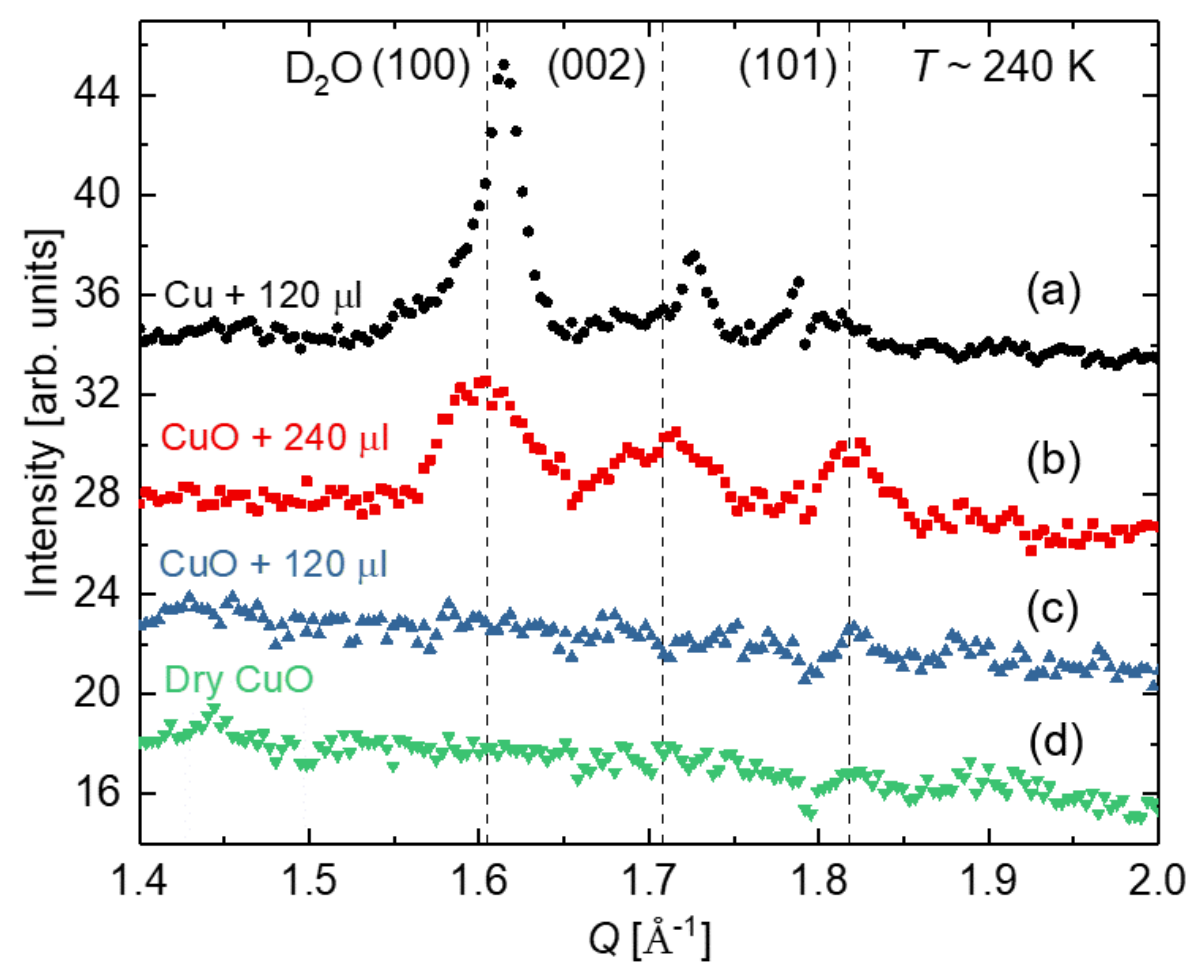

Figure 3.7. Neutron diffraction scans at $\sim 240 \mathrm{~K}$ vs. wave vector transfer $Q$ for four samples: (a) untreated-Cu foils with $120 \mu \mathrm{l}$ of $\mathrm{D}_{2} \mathrm{O}$ (black squares); (b) CuO-coated sample with $240 \mu 1$ of $\mathrm{D}_{2} \mathrm{O}$ (red squares); (c) CuO-coated sample with $120 \mu 1$ of $\mathrm{D}_{2} \mathrm{O}$ (blue up triangles); and (d) CuO-coated sample without added $\mathrm{D}_{2} \mathrm{O}$ (green down triangles). Vertical lines are drawn at $Q$ values corresponding to three Bragg reflections calculated for bulk hexagonal $\mathrm{D}_{2} \mathrm{O}$ ice at $240 \mathrm{~K}$ [136]: (100), (002), and (101). Patterns are offset vertically for clarity.

We then dismounted the sample, heated it in air at $\sim 328 \mathrm{~K}$ for 24 hours, and resealed it with $240 \mu$ of $\mathrm{D}_{2} \mathrm{O}$. A subsequent diffraction scan at $260 \mathrm{~K}$ (Fig. 3.7b) shows three Bragg peaks indicative of hexagonal ice similar to those observed in scans of the untreated-Cu sample (Fig. 3.7a). We interpret these results as indicating the growth of hexagonal ice at higher $\mathrm{D}_{2} \mathrm{O}$ coverage. No Bragg peaks that could be identified with cubic ice were present in repeated scans of the nanostructured samples. In this respect, our results differ from measurements on water confined to mesoporous silica where 
evidence of cubic as well as hexagonal and disordered ice have been found $[\mathbf{9 4 , 1 2 6}]$. The larger width of the peaks in Fig. 3.7b compared to those in Fig. 3.7a indicates a smaller crystallite size and/or larger mosaic structure than for the ice on the untreated-Cu sample - a feature that may be caused by the water film thickness being less than the height of the $\mathrm{CuO}$ nanostructures as discussed earlier. From the observed broadening of the $\mathrm{D}_{2} \mathrm{O}$ (100) peak below $260 \mathrm{~K}$, the average domain size of the ice particles is estimated to be $\sim 30 \mathrm{~nm}$ based on a Scherrer analysis. A more detailed analysis of the ice peaks is presented in Appendix C.

It is well known that metal oxide surfaces exposed to humid air adsorb molecular water, which remains on the surface or dissociates into hydroxyl $(-\mathrm{OH})$ groups [132]. The hydroxide reactions at the copper surface that take place during sample preparation (Eqs. (2.1a) and (2.1b)) are also likely sources of $-\mathrm{OH}$. The $\mathrm{H}$ atoms in these surface components will scatter neutrons incoherently, producing an isotropic background in diffraction measurements. Consistent with this effect, we found that the background intensity of the CuO-coated samples was greater than that of the bare $\mathrm{Cu}$ samples by about a factor of two and slightly higher in the low- $Q$ region $\left(<0.5 \AA^{-1}\right)$ due to smallangle scattering (see Fig. C.4).

The absence of Bragg peaks at lower $\mathrm{D}_{2} \mathrm{O}$ coverages could be explained by water initially solidifying into an amorphous or glassy structure which lacks long-range order. We suggest that the most favorable candidate for amorphous solid water would be the first water population identified in the elastic FWS of both the wet and dry $\mathrm{CuO}$ samples (Fig. 3.3a and 3.3b, respectively), whose intensity has the smaller slope on cooling. Because it is the first to immobilize, indicating the strongest interaction with the $\mathrm{CuO}$ 
nanostructures, this population should be the most likely to form a distorted network of hydrogen bonds that is incompatible with crystalline ice. This interpretation is consistent with our ESEM images (e.g., Fig. 2.12), which show water to condense first near the base of the nanostructures where the density of the $\mathrm{CuO}$ blades is the highest. However, it is possible that the second water population identified in the FWS of the wet $\mathrm{CuO}$ sample might also freeze into an amorphous structure. Its higher level of hydration $\left(60 \mu \mathrm{l}\right.$ of $\mathrm{D}_{2} \mathrm{O}$ added) is still less than that at which the Bragg peaks of hexagonal ice appeared (240 $\mu 1)$. More importantly, the hydration level of $60 \mu \mathrm{l}$ is less than the hydration level of $120 \mu \mathrm{l}$ at which no crystalline ice was observed. It is interesting to note that previous investigations $[\mathbf{9 4 , 1 2 6}]$ of water freezing in mesoporous silicas have presented evidence of disordered ice that forms in a layer adjacent to the pore walls in which the local hydrogen bonding of the water molecules is believed to differ from that of bulk ice. Similarly, Mamontov et al. [127] concluded that water adsorbed in partially filled pores of their ultra-microporous carbon samples did not crystallize on cooling, which they attributed to a disruption of its hydrogen bonding network.

In Fig. 3.8a, we show diffraction patterns of the CuO-coated sample hydrated with $240 \mu l$ of $\mathrm{D}_{2} \mathrm{O}$ at a series of temperatures taken subsequently to the pattern in Fig. 3.7b. As before, the Bragg peak positions are consistent with bulk hexagonal $\mathrm{D}_{2} \mathrm{O}$ ice [136]. In addition, we find that the intensity of the three Bragg peaks does not increase abruptly but rather gradually as the sample is cooled to $200 \mathrm{~K}$. This behavior can be seen more clearly in Fig. 3.8b where we have plotted the integrated intensity of the $\mathrm{D}_{2} \mathrm{O}$ (100) peak vs. temperature. As indicated by the dashed line, the peak intensity increases linearly from a temperature at $\sim 260 \mathrm{~K}$ at which ice peaks first appear down to $\sim 230 \mathrm{~K}$ 
where the intensity levels-off, indicating that all the bulk-like water has crystalized. From our Scherrer analysis, the width of the (100) peak is approximately constant over this temperature range from which we can conclude that ice growth on the $\mathrm{CuO}$ surfaces proceeds through nucleation of additional ice particles rather than by coalescence.

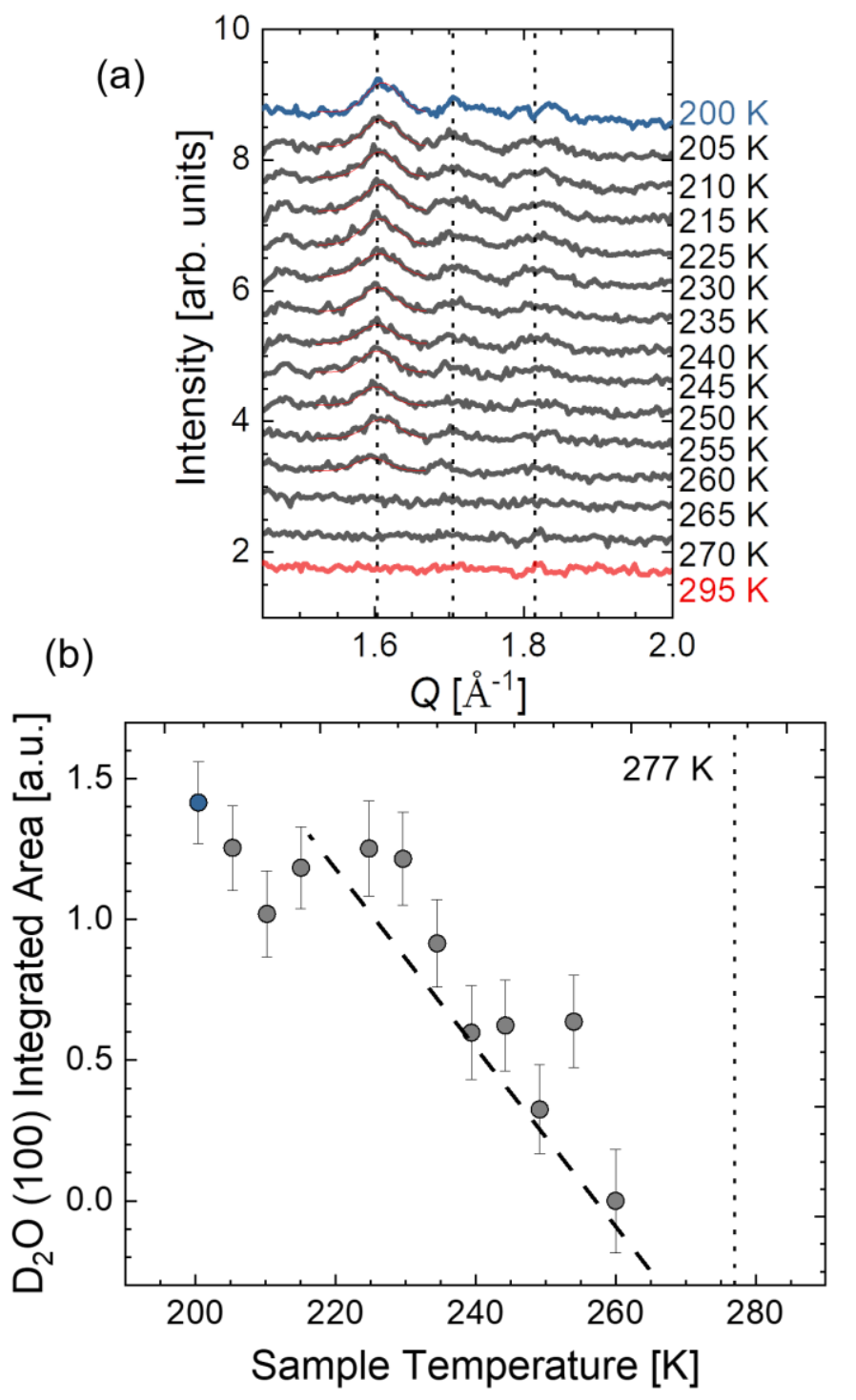

Figure 3.8. (a) Diffraction scans of the $\mathrm{CuO}$ sample hydrated with $240 \mu \mathrm{l}$ of $\mathrm{D}_{2} \mathrm{O}$ as a function of temperature taken every $5 \mathrm{~K}$ on cooling from $295 \mathrm{~K}$ to $200 \mathrm{~K}$. Vertical dotted lines are drawn at the calculated positions of the first three most intense Bragg peaks associated with hexagonal ice at $265 \mathrm{~K}$ [136]. (b) Temperature dependence of the $\mathrm{D}_{2} \mathrm{O}(100)$ Bragg peak integrated intensity normalized to the peak at $260 \mathrm{~K}$ determined from a Gaussian fit to the peaks in (a). The dashed line is a least-squares fit to the data between $230 \mathrm{~K}$ and $260 \mathrm{~K}$. Error bars represent one standard deviation in the uncertainty of the intensity. A vertical dotted line is drawn at the bulk melting point of $\mathrm{D}_{2} \mathrm{O}, 277 \mathrm{~K}$. 
The gradual increase in the amount of crystalline ice in Fig. 3.8b appears to be related to the continuous freezing observed in our FWS (Fig. 3.3); but, as these samples are hydrated to different levels and measured on different types of instruments, it is virtually impossible to compare the slopes of the linear terms directly. Still, this comparison would be interesting, recalling that an increase in the elastic intensity of the FWS is proportional to the amount of immobilized water - moving on a time scale $>4 \mathrm{~ns}$ - whereas the Bragg intensity is proportional to the amount of crystallized water.

A possible explanation for the gradual increase in the amount of ice with decreasing temperature in Fig. 3.8b is the conversion of non-crystalline ice (e.g., amorphous solid water or supercooled liquid) into small crystallites. This suggestion is based on studies of water confined within $\sim 86 \AA$ diameter cylindrical pores of templated silica (SBA-15) $[\mathbf{9 4 , 1 3 4 ]}$. Results from NMR cryoporometry and temperature-dependent neutron and x-ray diffraction by Webber et al. [94] and Jelassi et al. [69] indicate the presence of three phases of water within the silica pores: hexagonal and cubic ice as well as a disordered ice phase. The latter exhibited dynamics analogous to plastic phases in organic materials, i.e., the molecules form a well-defined lattice structure but are rotationally disordered. Furthermore, the disordered ice converted to crystalline (cubic) ice on cooling. Further measurements are required to determine whether a similar reversible transformation to crystalline ice is occurring at high levels of hydration in our CuO-coated samples.

As discussed earlier, the Bragg peak intensity in Fig. 3.8b increased as the temperature decreased due to an increase in the number of ice crystals forming near $\mathrm{CuO}$ nanostructures rather than by an increase in the grain size of already-nucleated ice. Thus, 
in the $\mathrm{CuO}$ system, we hypothesize that increasing the hydration level from $120 \mu \mathrm{l}$ to 240 $\mu 1$ of $\mathrm{D}_{2} \mathrm{O}$ introduces bulk-like ice particles located further from the substrate where the density of the nanostructures is lower and where hexagonal ice crystals of about the same size can nucleate on cooling. 


\section{Chapter 4}

\section{Dynamics of Interfacial Water Near}

\section{Hydrophilic CuO-Nanostructured Surfaces}

As deduced from our elastic fixed-window scans presented in Sec. 3.3, the interfacial water near $\mathrm{CuO}$ nanostructures undergoes a continuous freezing transition that spans the temperature range 200-280 K. In Sec. 3.4, we showed that, while we had sufficient sensitivity, we did not observe Bragg peaks characteristic of crystalline ice in repeated neutron diffraction measurements on CuO-coated samples hydrated with up to $120 \mu \mathrm{l}$ of $\mathrm{D}_{2} \mathrm{O}$. On this basis, we concluded that the water in our CuO-coated samples freezes continuously into an amorphous solid. Nevertheless, it would be desirable to have positive evidence for amorphous solid water rather than only a null result from diffraction measurements. For this reason, we have conducted quasielastic (QENS) and inelastic neutron scattering (INS) measurements to determine how the dynamics (translational, rotational, and vibrational modes) of interfacial water differs from that of bulk water.

Based on the vibrational dynamics presented in Sec. 4.1, we show that at a temperature of $5 \mathrm{~K}$ the collective vibrational modes (phonons) of the solid water are significantly reduced compared to those of bulk ice. In addition, we observe that the characteristically sharp edge of bulk ice's librational band is broadened toward lower energy transfers, indicating that the $\mathrm{H}$-bonds between water molecules have been weakened. These observations can be explained by the water-CuO surface interaction 
being stronger than the water-water interaction, leading to a distorted hydrogen bonding network compared to bulk ice.

The elastic scans presented in Chapter 3 have provided little information regarding the dynamics of the mobile water in the continuous freezing transition. Therefore, we investigated the diffusivity of the interfacial water on a $0.03-1 \mathrm{~ns}$ time scale in the temperature range 230-270 K using QENS as discussed below in Sec. 4.2.

\subsection{Inelastic Neutron Scattering from Water Near Hydrophilic CuO-Nanostructured Surfaces}

Inelastic neutron scattering allows investigation of the vibrational dynamics and molecular interactions of liquids and solids. The exceptionally large incoherent cross section of hydrogen makes neutrons highly sensitive to hydrogen nuclei in the thin layer of water located near the nanostructured $\mathrm{CuO}$ surfaces. Furthermore, the absence of selection rules gives neutrons an advantage in obtaining the complete vibrational density of states (VDOS) compared to optical methods such as Raman and infrared spectroscopy. Therefore, we collected vibrational spectra for our hydrated $\mathrm{CuO}$ samples, using the timeof-flight spectrometer VISION at ORNL's SNS [141].

VISION is an inverted-geometry crystal-analyzer spectrometer that is optimized to investigate molecular vibrations, chemical bonding, and intermolecular interactions with its high energy resolution $(<1.5 \% \Delta E / E$ for $E>2 \mathrm{meV})$ and dynamic range of 5-600 meV. We used the same CuO-coated samples hydrated with $60 \mu \mathrm{l}$ and $10 \mu \mathrm{l}$ of $\mathrm{H}_{2} \mathrm{O}$ as previously measured on the HFBS (see Sec. 3.2). In addition, two dried samples of bare 
and $\mathrm{CuO}$-coated copper as well as a bulk water sample were measured for comparison. All samples were first quenched in liquid nitrogen and then cooled to $5 \mathrm{~K}$, the base temperature of the cryostat, in order to minimize thermal (Debye-Waller) effects. All spectra were reduced using standard tools at ORNL (see Appendix A for more details). Our aims were two-fold: (1) to investigate the vibrational dynamics of solid interfacial water near $\mathrm{CuO}$ nanostructures compared to bulk ice; and (2) to determine the type of water (crystalline vs. amorphous) at the $\mathrm{CuO}$ interface based on the observed vibrational dynamics.

The sample of bulk water $\left(\mathrm{H}_{2} \mathrm{O}\right)$ consisted of 2.8 grams of deionized water sealed in a standard flat aluminum cell $(0.5 \mathrm{~mm}$ thick $)$. The vibrational spectra of bulk water are plotted in Fig. 4.1 over energy transfers of 0-150 meV and temperatures of 5-300 K. On cooling from $300 \mathrm{~K}$, the initially broad band centered around $60 \mathrm{meV}$ becomes narrower and develops some structure below $50 \mathrm{~K}$, especially near the low-energy cutoff (edge) near $\sim 67 \mathrm{meV}$. The sharpness of the edge of this broad band signifies a well-defined minimum energy required to excite the vibrations. At lower energies, several peaks emerge and become shaper as the sample is cooled down to the lowest temperature of 5 K.

The vibrational modes of bulk crystalline ice have been identified and analyzed previously [142-143]. A defining feature of the bulk ice vibrational spectrum is the characteristically sharp edge at $\sim 67 \mathrm{meV}$ on the low energy side of the librational band, which consists of localized, hindered rotational modes (wagging, twisting, and rocking) of the water molecule about its three principal axes (see insets in Fig. 4.1). These modes are highly sensitive to local distortions in the H-bonds between water molecules. Below 
$50 \mathrm{meV}$ are collective vibrational modes, including the hindered translational (acoustic) mode at $7.1 \mathrm{meV}$ followed by two optical modes (H-bond stretching) centered at 28.4 and $37.9 \mathrm{meV}$, respectively.

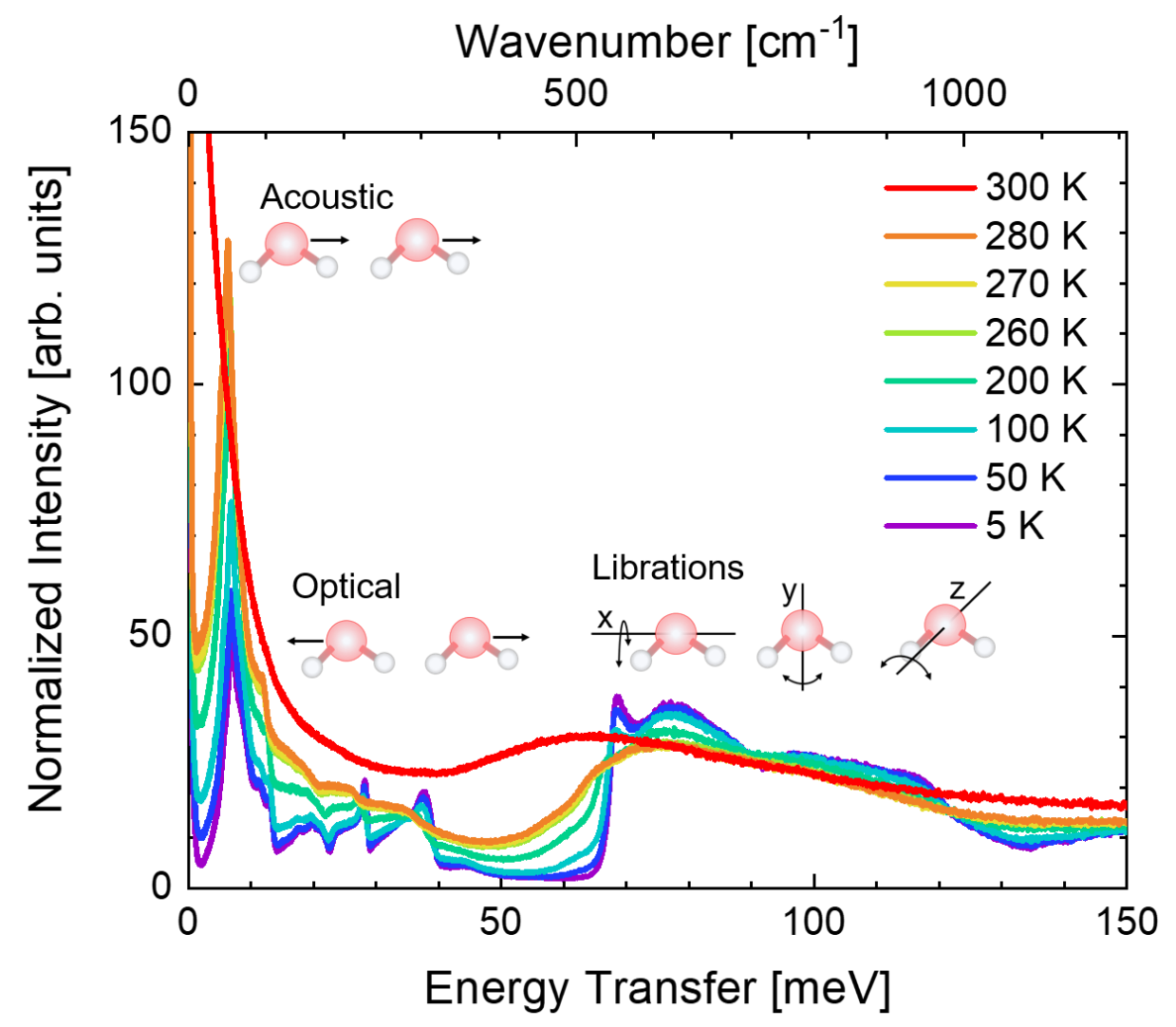

Figure 4.1. Inelastic neutron scattering (INS) spectra of bulk water at various temperatures at low energy-transfers $(E<150 \mathrm{meV})$.

Plotted in Fig. 4.2 are the vibrational spectra measured at $5 \mathrm{~K}$ for our three $\mathrm{CuO}$ samples hydrated with different amounts of $\mathrm{H}_{2} \mathrm{O}: 60 \mu \mathrm{l}$ (wet), $10 \mu \mathrm{l}$ (dry), and $0 \mu \mathrm{l}$ (vacuum dried for $24 \mathrm{hrs}$.$) . The last sample (\mathrm{CuO}+0 \mu \mathrm{l})$ represents a dry state of the $\mathrm{CuO}$ surface prior to adding water to the sample cell. We also measured the vibrational spectrum for a bare-Cu sample without a $\mathrm{CuO}$ coating to help identify copper phonons. As shown in Fig. 4.2, there are a number of optical modes attributed to copper below $\sim 45$ meV, which might obscure any peaks contributed by water. 


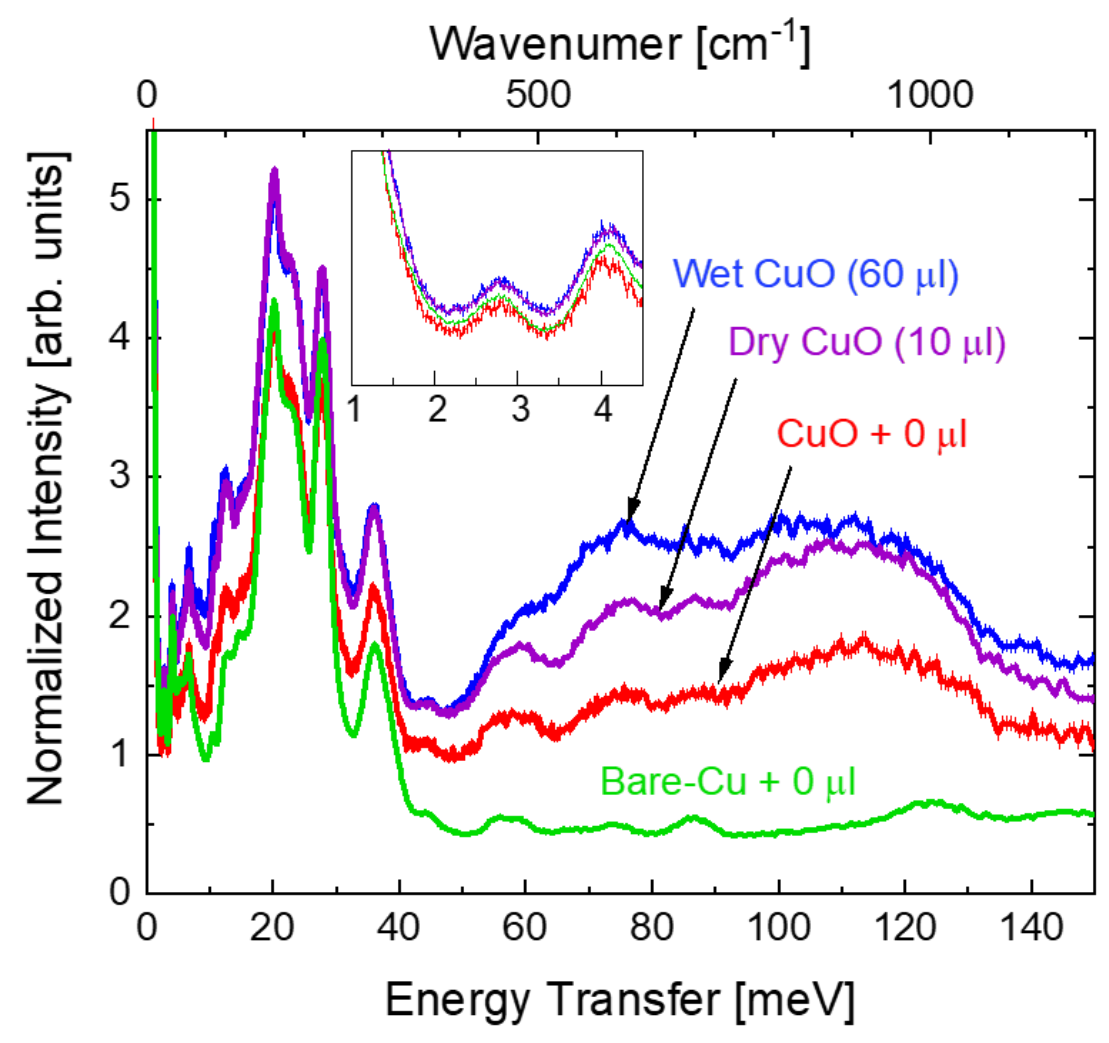

Figure 4.2. Monitor-normalized vibrational spectra measured at $5 \mathrm{~K}$ in the low energytransfer range for our four samples: $\mathrm{CuO}$-coated copper foils hydrated with $60 \mu \mathrm{l}$ of added $\mathrm{H}_{2} \mathrm{O}$ (blue); a similar sample with $10 \mu \mathrm{l}$ of $\mathrm{H}_{2} \mathrm{O}$ (purple); a similar sample without any added water (red); and, untreated copper foils without any added water (green).

In order to compare the scattered spectral intensities observed for different samples, all spectra were normalized to the copper phonon peak centered around $\sim 3 \mathrm{meV}$ (see inset in Fig. 4.2). This peak was chosen because it lies in a minimum in the bulk water spectrum at $5 \mathrm{~K}$ and thus less likely to be affected by the different amount of water added to each sample. This normalization is especially important for our analysis of the librational band. The normalized spectra are plotted in Fig. 4.3. 


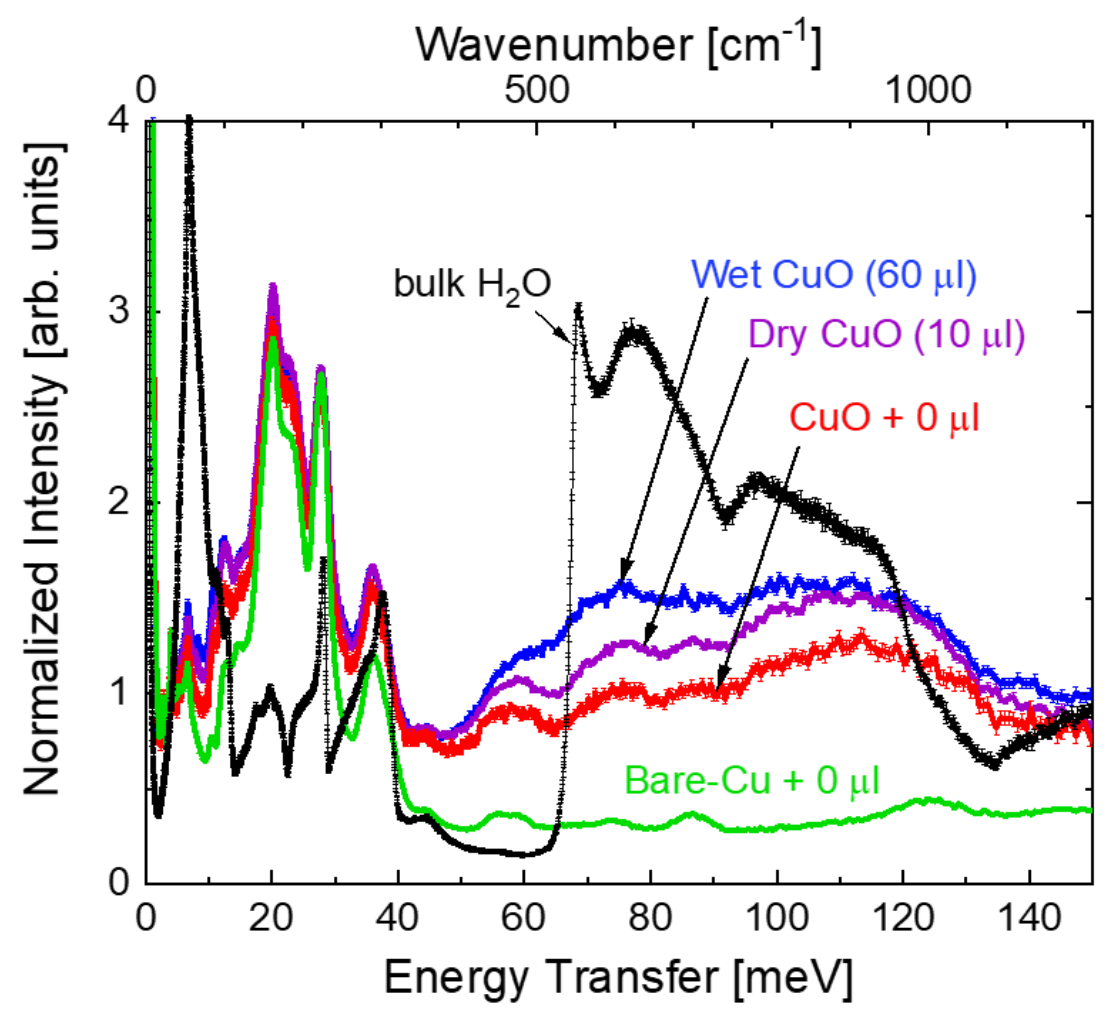

Figure 4.3. Vibrational spectra reproduced from Fig. 4.2 and now normalized to the copper phonon centered around $3 \mathrm{meV}$. Also included is a reference spectrum for bulk water, previously measured at $5 \mathrm{~K}$ on VISION, whose intensity has been arbitrarily scaled to fit within the plot window.

Comparing bare copper and nanostructured $\mathrm{CuO}(\mathrm{CuO}+0 \mu \mathrm{l})$ in Fig. 4.3, we see a significant increase in the scattered neutron intensity for energy transfers above $\sim 40$ meV. The origin of this difference is explained by the presence of strongly adsorbed water molecules on the oxide surface that were not removed after preparation of the $\mathrm{CuO}$ nanostructured $\mathrm{CuO}$. This explanation is consistent with the presence of adsorbed $\mathrm{H}_{2} \mathrm{O}$ identified in our neutron diffraction measurements by a significant increase in the background intensity compared to bare copper (see Fig. C.4). It is also consistent with the observed offset in the elastic intensity at $280 \mathrm{~K}$ measured on the HFBS between uncoated and CuO-coated copper (see Fig.3.3). 
To evaluate the differences in the librational band between the spectra of interfacial and bulk water, we require removal of the contribution to the spectra from the substrate. For this reason, in Fig. 4.4, the spectrum of vacuum-dried $\mathrm{CuO}$ sample $(\mathrm{CuO}+$ $0 \mu 1)$ has been subtracted from the spectra of the wet and dry $\mathrm{CuO}$-coated samples. The spectrum for bulk water is also plotted in Fig. $\mathbf{4 . 4}$ for comparison where its elastic intensity has been scaled to that of the wet $\mathrm{CuO}$ sample in the energy transfer range \pm 1 $\mathrm{meV}$. To check reproducibility, the spectrum of the wet sample (green) is also shown after it had been opened, vacuum dried at $100{ }^{\circ} \mathrm{C}$ for 24 hours, and sealed with another 60 $\mu \mathrm{l}$ of $\mathrm{H}_{2} \mathrm{O}$ under helium atmosphere. After this rehydration, the overall intensity decreased by $\sim 20-30 \%$ compared to its initial intensity, indicating less water in the scattering volume, but the positions and shapes of the peaks were unaffected.

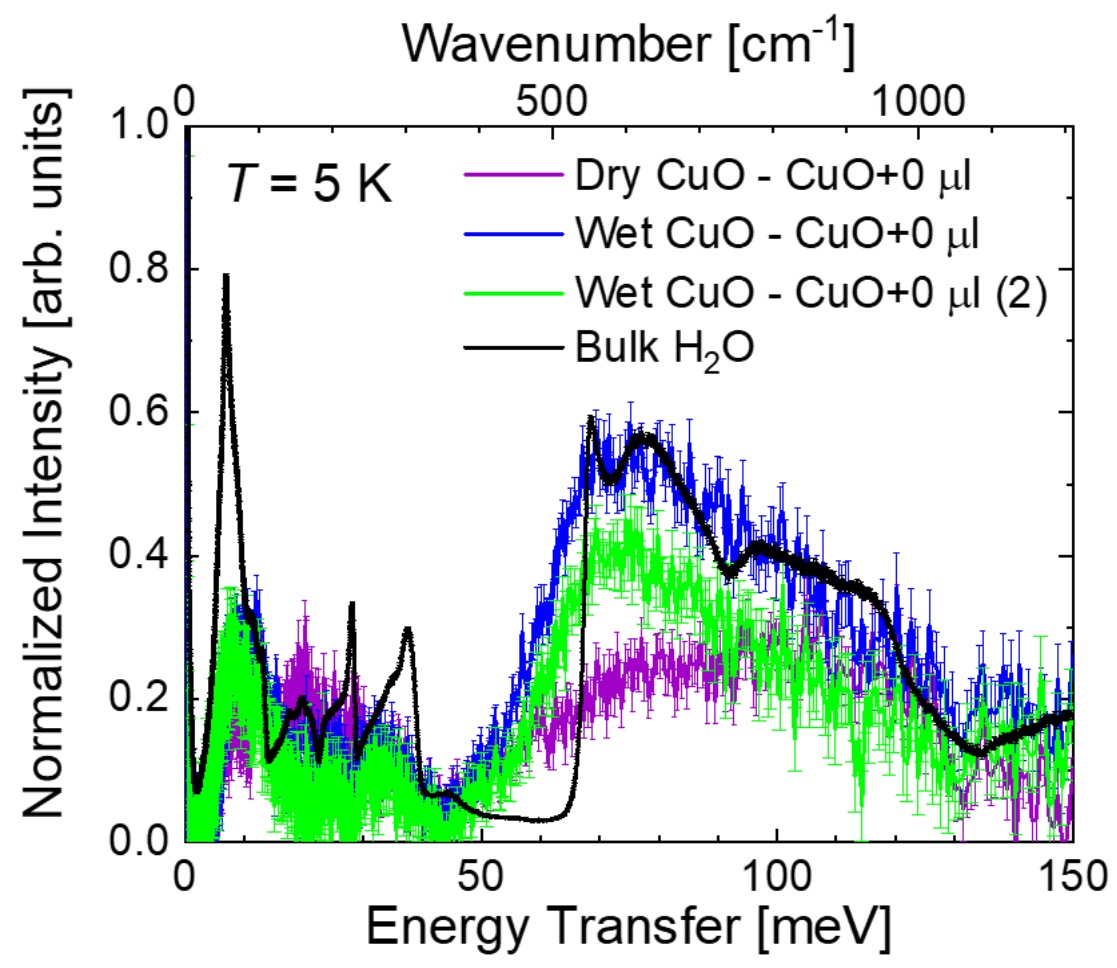

Figure 4.4. Difference spectra at $5 \mathrm{~K}$ of the wet (blue and green) and dry (purple) samples with the spectrum of the vacuum dried $\mathrm{CuO}$ sample $(\mathrm{CuO}+0 \mu \mathrm{l})$ subtracted. Also shown is a reference spectrum for bulk ice at $5 \mathrm{~K}$, which has been scaled such that its elastic intensity is equal to that of the wet $\mathrm{CuO}$ sample. 
As shown in Fig. 4.4, the intensity and shapes of the peaks below $50 \mathrm{meV}$ are different from those of bulk ice. In particular, the intensity of the acoustic mode at $7 \mathrm{meV}$ is markedly reduced in the $\mathrm{CuO}$ samples, indicating a loss of long-range translational order. Also, the center of the acoustic mode shifts slightly upward from $~ 7.1 \mathrm{meV}$ in bulk ice to $\sim 7.5 \mathrm{meV}$ and $\sim 10 \mathrm{meV}$ for the dry and wet $\mathrm{CuO}$ samples, respectively. We attribute the weakness of the acoustic modes to a strong interaction between adsorbed water and the $\mathrm{CuO}$ nanostructures as is believed to be the case for water tightly bound to $\mathrm{CuO}$ nanoparticles (15 $\mathrm{nm}$ diameter) [144]. Moreover, the shift in the optic band can be explained by so-called "riding modes" - correlated motion between the vibrations of the interfacial water and the optical modes of the underlying metal oxide surface [144].

In addition to the intensity loss of the low-energy collective modes, Fig. $\mathbf{4 . 4}$ shows a large intensity decrease of the librational band of the dry sample and a significant broadening of its leading edge. These features indicate that the hydrogen bonds between water molecules have been weakened due to the strong interaction with the $\mathrm{CuO}$ nanostructures [144-145]. The spectrum of the wet sample still shows some edge broadening; however, above $\sim 67 \mathrm{meV}$, the spectrum has a shape similar to that of bulk crystalline ice. This indicates that, locally, the H-bonding of the added water is structurally similar to bulk crystalline ice. As for the broadening of the librational edge, we note that the local tetrahedral H-bonding in bulk ice becomes rather distorted in amorphous ice, which is manifested in a similar shift of the libration band to lower energy transfers [143-145]. Amorphous ice-like vibrational dynamics also aptly describes hydration water of DNA [146-147] and some living cells and tissues [148]. Thus, we conclude from these INS measurements that the interfacial water in proximity to the $\mathrm{CuO}$ 
coating forms a short-ranged and distorted H-bonding network with features in the lowtemperature vibrational spectra that are similar to those of amorphous ice rather than bulk crystalline ice.

\section{Water Polyamorphism}

Water is polymorphic and exists in at least 18 different stable (and metastable) phases of crystalline ice [149]. In addition, water can also be prepared in one of three main polymorphs of amorphous ice: low-density (LDA), high-density (HDA), and veryhigh-density (VHDA) amorphous ice. We concluded from the previous section that interfacial water near $\mathrm{CuO}$ nanostructures solidifies into an amorphous structure. Therefore, this section discusses the various ways that have been used to prepare samples of amorphous ice. It must be noted that the properties of these amorphous "states" like density or their radial distribution function often vary depending on sample preparation and experimental protocols. For more details on the low-temperature amorphous states of water, the reader is directed to several recent review articles and references cited therein $[86,150-152]$.

Burton and Oliver [153] were among the first to prepare amorphous solid water (ASW) by depositing water vapor on cryo-cooled ( $80 \mathrm{~K})$ substrates. Annealing ASW to $\sim 110 \mathrm{~K}$ removes micropores and creates a reproducible state of ASW [152]. In the 1980’s, Brüggeller and Mayer [154] vitrified micron-sized droplets of water by quenching them in a liquid cryomedium (e.g., liquid ethane). A similar process of creating this so-called "hyper-quenched glassy water (HGW)" [86] or "vitreous ice" is now commonly used in electron microscopy. Creation of HGW relies on bypassing the 
homogeneous nucleation of ice $(\sim 231-235 \mathrm{~K}$ at 1 bar $[\mathbf{8 4 , 1 5 2}])$ by cooling at rates in excess of $10^{6} \mathrm{~K} / \mathrm{s}$.

High-density amorphous ice (HDA) is commonly prepared by applying pressure $(>0.8 \mathrm{GPa})$ to hexagonal ice at liquid nitrogen temperatures [155], which results in HDA that can be recovered by isothermal decompression to atmospheric pressure. Furthermore, one can convert the HDA to a low-density state (LDA) by heating above $115 \mathrm{~K}$ at atmospheric pressure where the LDA has a density of about $0.94 \mathrm{~g} / \mathrm{cm}^{3}$ [152]. We note that recent work by Tulk et al. [156] challenges the current understanding of the transition from HDA to, and from, hexagonal ice. Very-high-density amorphous ice (VHDA) can be formed by isobaric heating of HDA (above $1 \mathrm{GPa}$ ) from $77 \mathrm{~K}$ to $\sim 165 \mathrm{~K}$ [157]. Structurally, the polymorphs ASW and HGW are indistinguishable from LDA at the atomic level where all three have identical densities and contain four nearest neighbors in the first coordination shell $[\mathbf{1 5 2}, \mathbf{1 5 7}]$. By slow heating from cryogenic temperatures and at atmospheric pressure, all forms of amorphous ice crystallize at temperatures near $150 \mathrm{~K}$ [84], which marks the lower limit of "no man's land" as discussed in Sec. 1.3.2. Table 4.1 summarizes the commonly prepared forms of amorphous ice and their densities.

\begin{tabular}{ccc} 
Amorphous ice & Density ${ }^{\mathrm{a}}\left[\mathrm{g} / \mathrm{cm}^{3}\right]$ & Ref. \\
\hline ASW & 0.94 & $\mathbf{1 5 2}$ \\
HGW & 0.94 & $\mathbf{1 5 2}$ \\
LDA & $0.94 \pm 0.02$ & $\mathbf{1 5 1}$ \\
HDA & $1.17 \pm 0.02$ & $\mathbf{1 5 1}$ \\
VHDA & $1.25 \pm 0.01$ & $\mathbf{1 5 1}$
\end{tabular}

Table 4.1. Various types of amorphous ice produced experimentally. ${ }^{a}$ Density measured at $77 \mathrm{~K}$ at atmospheric pressure (1 bar). 


\subsection{Quasielastic Neutron Scattering from Water Near Hydrophilic CuO-Nanostructured Surfaces}

In Chapter 3, we investigated the continuous liquid-solid phase transition and the possibility of amorphous solid water in proximity to superhydrophilic $\mathrm{CuO}$ nanostructures. The inelastic neutron scattering measurements described in the previous section support the presence of amorphous solid water in our samples. They provided evidence of $\mathrm{H}$-bonding distorted from that of bulk ice between water molecules that are strongly interacting with the $\mathrm{CuO}$ nanostructures. As yet, we have not discussed the motion of water molecules as they participate in the continuous freezing transition observed in our samples. Knowledge of the temperature dependence of the diffusive motion of water molecules could help interpret the elastic scans in Fig. 3.3. For example, it would be of interest to address the question of whether the two populations of water identified in analysis of the FWSs (see Sec. 3.3) are characterized by different diffusion rates as our group had found for single-supported bilayer lipid membranes $[\mathbf{1 2 9}, \mathbf{1 5 8}]$. From a technological perspective, the molecular level dynamics might ultimately provide insight into the appropriate boundary condition for a liquid slug moving over a $\mathrm{CuO}$ coated surface inside an OHP. Therefore, in this section, we discuss our investigation of the water dynamics near a CuO-coated surface using QENS measurements performed on two complementary backscattering spectrometers (see Sec. 3.1 for more details). The fixed-window scans in Sec. 3.3 provide insight into the appropriate temperatures to measure the QENS signal. Accordingly, these scans are reproduced in Fig. 4.5 to facilitate discussion. 


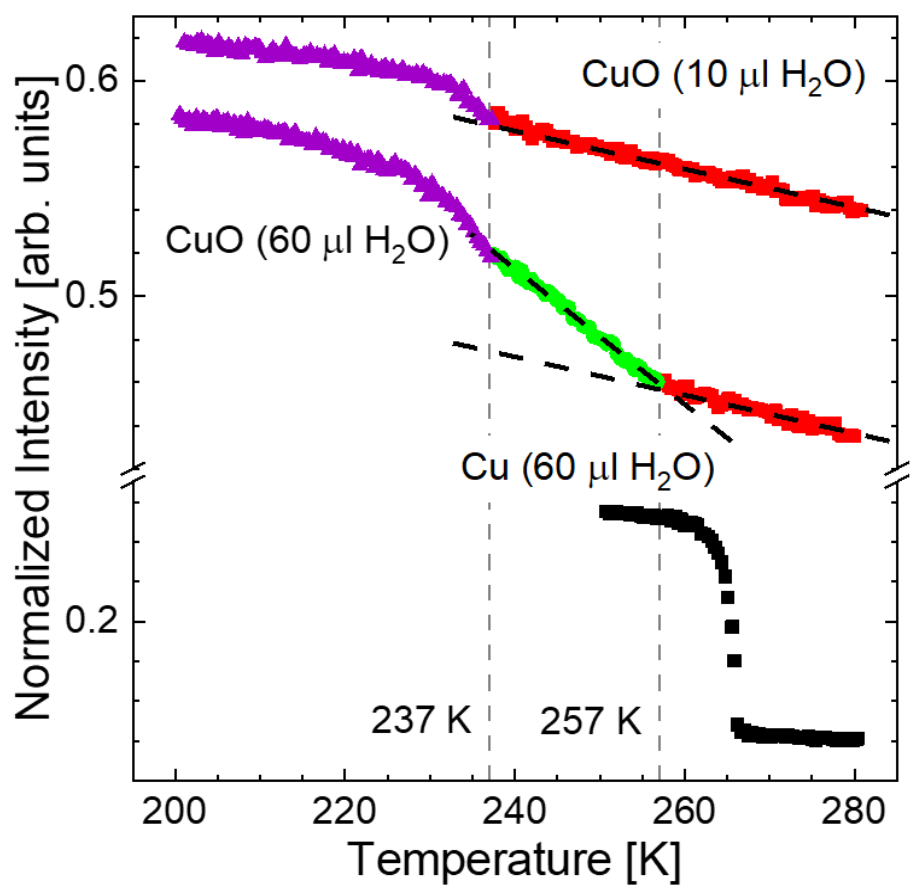

Figure 4.5. FWS reproduced from Fig. 3.3 excluding the detector at $Q=0.25 \AA^{-1}$ in order to reduce the coherent scattering from $\mathrm{Cu}$ the substrate. Data were collected on the HFBS and used to determine temperatures at which to collect QENS spectra on the HFBS and BaSiS. Vertical dashed lines indicate inflection points in the elastic neutron intensity. Regions with similar freezing behavior are highlighted with the same colors.

Recall that an increase in the elastic intensity as measured on the HFBS is proportional to the increase in the number of water molecules that move on a timescale longer than $\sim 4$ ns. As discussed in Sec. 3.3, we interpreted the initial linear temperature dependence (red data points in Fig. 4.5), common to both the wet and dry samples, as representing a population of water molecules strongly bound to the $\mathrm{CuO}$ nanostructures. The addition of $50 \mu \mathrm{l}$ of $\mathrm{H}_{2} \mathrm{O}$ was interpreted as introducing a second population of water (green points in Fig. 4.5) likely located in a region of the $\mathrm{CuO}$ coating with a lower density of nanostructures (e.g., further up a $\mathrm{CuO}$ blade). These elastic scans suggested obtaining QENS spectra at $270 \mathrm{~K}$ where the two samples share a common freezing behavior, at $250 \mathrm{~K}$ where the water in the wet sample freezes more rapidly on cooling, and at $230 \mathrm{~K}$ where most of water has been immobilized. 


\subsubsection{QENS Measured on HFBS}

QENS spectra were measured at a temperature of $250 \mathrm{~K}$ on HFBS for a sample of CuO-coated copper foils hydrated with $60 \mu 1 \mathrm{H}_{2} \mathrm{O} \mathrm{CuO}$ immediately after collecting FWS (Fig. 4.5). The temperature of $250 \mathrm{~K}$ was chosen for a feasibility measurement because it lies roughly in the midpoint of the continuous freezing transition and thus likely to have appreciable quasielastic scattering within the dynamic range of the HFBS $( \pm 17 \mu \mathrm{eV})$. A QENS spectrum from this experiment is shown in Fig. 4.6 at a wave-vector transfer $Q$ value of $\sim 0.7 \AA^{-1}$. We found that the spectra measured on the HFBS could be fit well by assuming a dynamic structure factor $S(Q, \omega)$ consisting of a sum of two terms: an elastic component described by a delta function plus a single Lorentzian function, representing the quasielastic scattering. As we will show in the next section, due to the larger dynamic range of BaSiS compared to HFBS, the QENS spectra measured on BaSiS can be modeled by a similar scattering law with an addition Lorentzian term.

$$
S(Q, \omega)=A(Q) \delta(\omega)+B(Q) \mathcal{L}_{N}(Q, \omega)+C(Q) \mathcal{L}_{B}(Q, \omega)
$$

Here, $A(Q), B(Q)$, and $C(Q)$ are free parameters; $Q=\left|\boldsymbol{k}_{i}-\boldsymbol{k}_{f}\right|$ is the neutron wave vector transfer; $\hbar \omega=E_{i}-E_{f}$ is the energy transfer to the neutron on scattering; and, $\mathcal{L}$ is a Lorentzian function given by Eq. (4.2) where $\Gamma$ is its half-width at half-maximum (HWHM). The subscripts $N$ and $B$ denote "narrow" and "broad," respectively.

$$
\mathcal{L}(Q, \omega)=\frac{1}{\pi} \frac{\Gamma(Q)}{[\Gamma(Q)]^{2}+\omega^{2}}
$$


The observed quasielastic intensities at each $Q$ value were fit to the intensity function $I(Q, \omega)$, which is obtained by convoluting Eq. (4.1) with the instrument resolution function $R(Q, \omega)$,

$$
I(Q, \omega)=S(Q, \omega) \otimes R(Q, \omega)+V(Q, \omega)
$$

Here, $V(Q, \omega)=a(Q) \omega+b(Q)$ is a linear background term. Data were fit using the Data Analysis and Visualization Environment (DAVE) software [159],

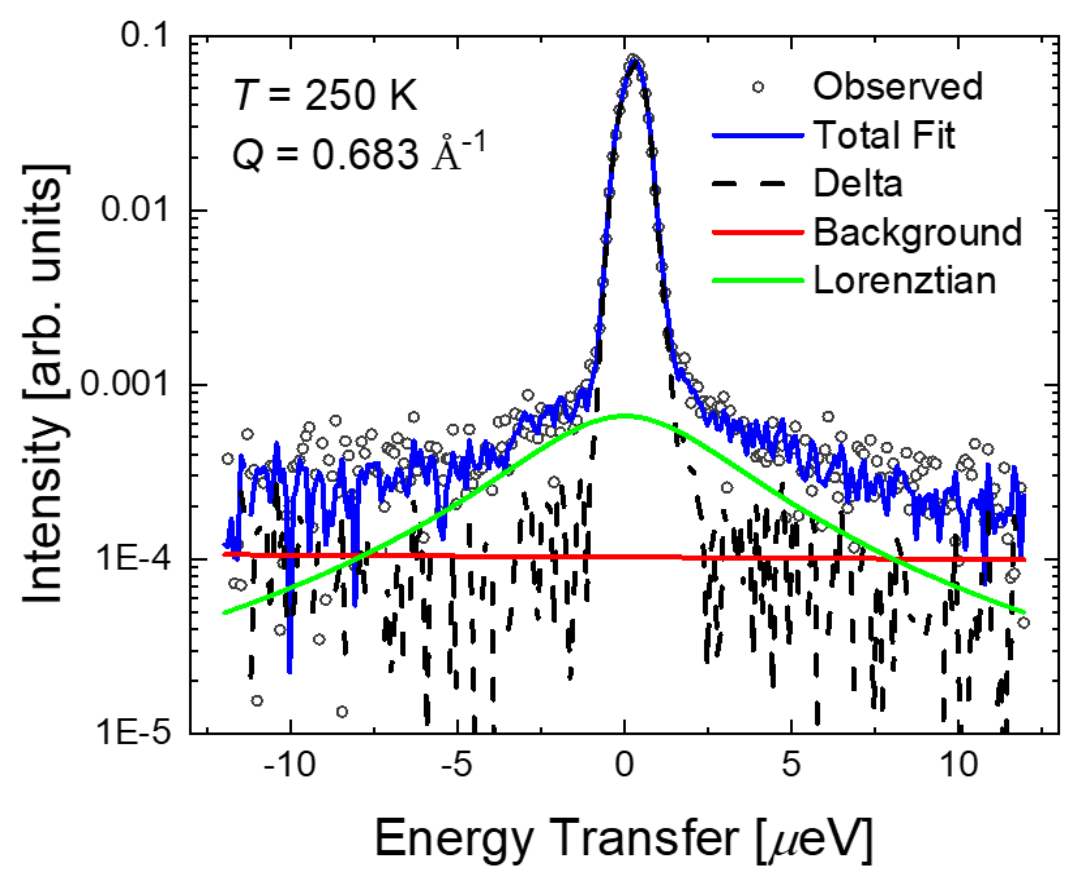

Figure 4.6. QENS spectrum of the wet CuO sample at $Q \sim 0.7 \AA^{-1}$ measured on the HFBS after cooling to $250 \mathrm{~K}$ at $0.08 \mathrm{~K} / \mathrm{min}$.

As indicated by the dashed curved in Fig. 4.6, there is a significant amount of elastic scattering compared to quasielastic scattering (green curve) at $250 \mathrm{~K}$. Note that in the $Q$ range of this experiment there should be no contribution to the elastic intensity from Bragg scattering from the copper substrate. Because the incoherent scattering in this experiment is dominated by the $\mathrm{H}$ atoms, the elastic component arises mainly from the presence of immobile water and possibly surface hydroxyls $(-\mathrm{OH})$. 
Unbounded Brownian-type diffusion of weakly interacting particles can be described by Fick's law given by $\Gamma(Q)=D Q^{2}$ where the factor $D$ is the translational diffusion coefficient [160], which has units of $\mathrm{cm}^{2} / \mathrm{s}$. The dynamics of bulk water have been extensively studied by QENS (see Ref. 161 and references cited therein). These studies have found that Fick's law only holds at low $Q\left(<1 \AA^{-1}\right)$ and $\Gamma$ tends to bend over at higher $Q$ as shown in Fig. 4.7. Furthermore, at high $Q, \Gamma$ decreases as the temperature of water decreases below $\sim 293 \mathrm{~K}$. It has been shown that this behavior can be described by a random jump-diffusion model in which $\Gamma(Q)$ is expressed as

$$
\Gamma(Q)=\frac{D Q^{2}}{1+D Q^{2} \tau_{0}}
$$

where $\tau_{0}$ is a characteristic residence time. 


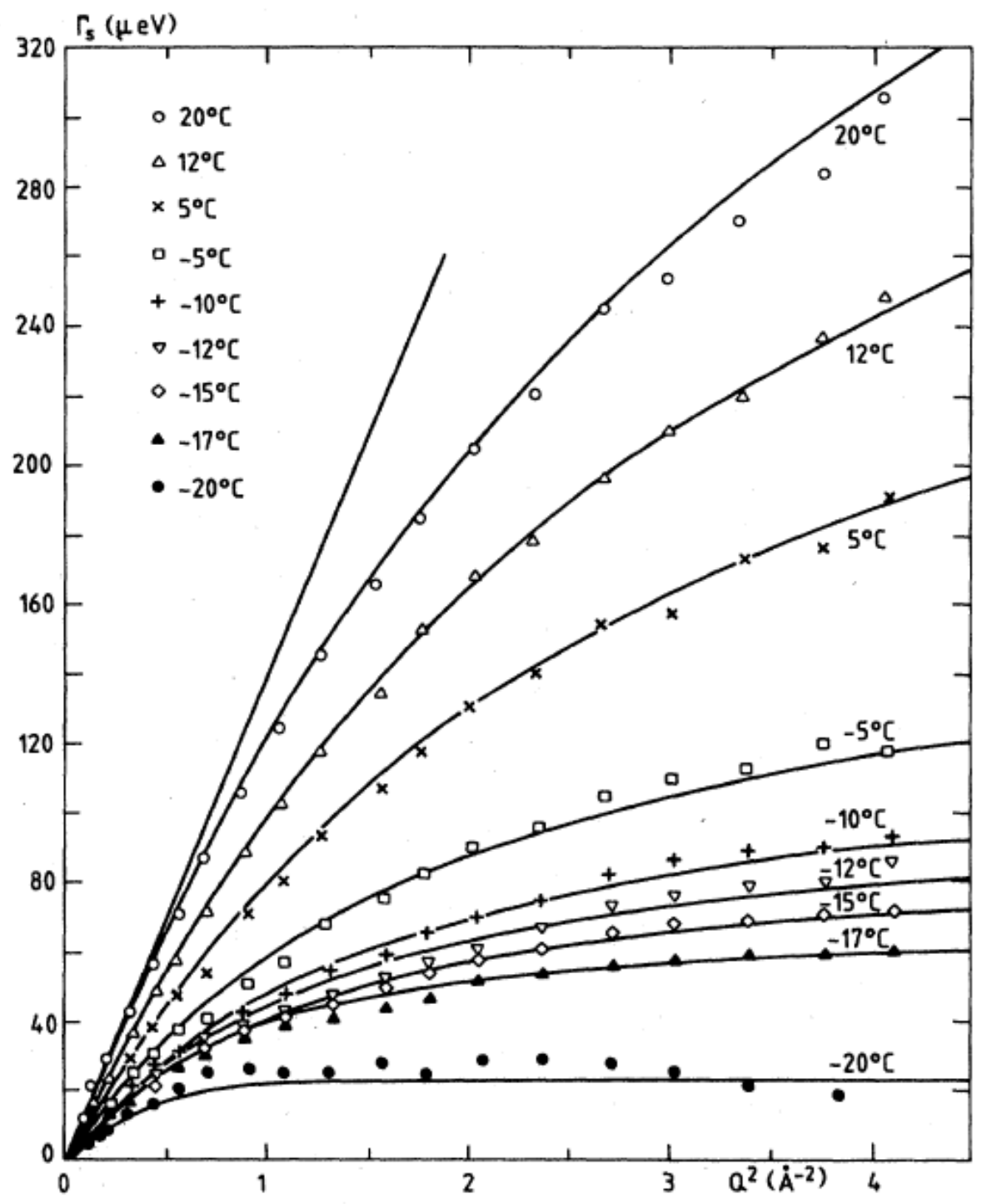

Figure 4.7. HWHM $\Gamma$ of the quasielastic peak vs. $Q^{2}$ for bulk water $\left(\mathrm{H}_{2} \mathrm{O}\right)$ as measured previously by Teixeira et al. [161] on the IN6 spectrometer at the Institut Laue Langevin at various temperatures. The energy resolution of the spectrometer (IN6) was $\Delta E=100$ $\mu \mathrm{eV}$ at the time of the experiment. Reproduced from Ref. [161].

In Fig. 4.8, we have plotted $\Gamma(Q)$ vs. $Q^{2}$ for the water in our wet $\mathrm{CuO}$ sample $(60$ $\mathrm{ml} \mathrm{H}_{2} \mathrm{O}$ added) at a temperature of $250 \mathrm{~K}$. Because it shows a behavior qualitatively similar to that of bulk water in Fig. 4.7, we fit $\Gamma(Q)$ to the expression in Eq. (4.4) for the random jump-diffusion model. The best fit is shown by the dashed red curve in Fig. $\mathbf{4 . 8}$ and yields a diffusion coefficient $D$ and residence time of $0.18 \pm 0.06 \times 10^{-5} \mathrm{~cm}^{2} / \mathrm{s}$ and 
$86.9 \pm 15.6$ ps, respectively. A fit of the low- $Q$ data $\left(<0.5 \AA^{-2}\right)$ to Fick's law yields $D \sim$ $0.11 \pm 0.04 \times 10^{-5} \mathrm{~cm}^{2} / \mathrm{s}$, which is a factor of about four slower than bulk supercooled water at $250 \mathrm{~K}$ [161]. We believe the Fick's law estimate of $D$ is more reliable as there is no reason to believe that a jump diffusion model should be applicable to the interfacial water in our CuO-coated samples. Despite the large uncertainty in $D$, we conclude that the diffusivity of the interfacial water at $250 \mathrm{~K}$ is significantly reduced compared to bulk supercooled water at a similar temperature. Unfortunately, no additional time was available on the HFBS to improve the statistics in Fig. 4.8 or to collect QENS at other temperatures in the continuous freezing region in Fig. 4.5.

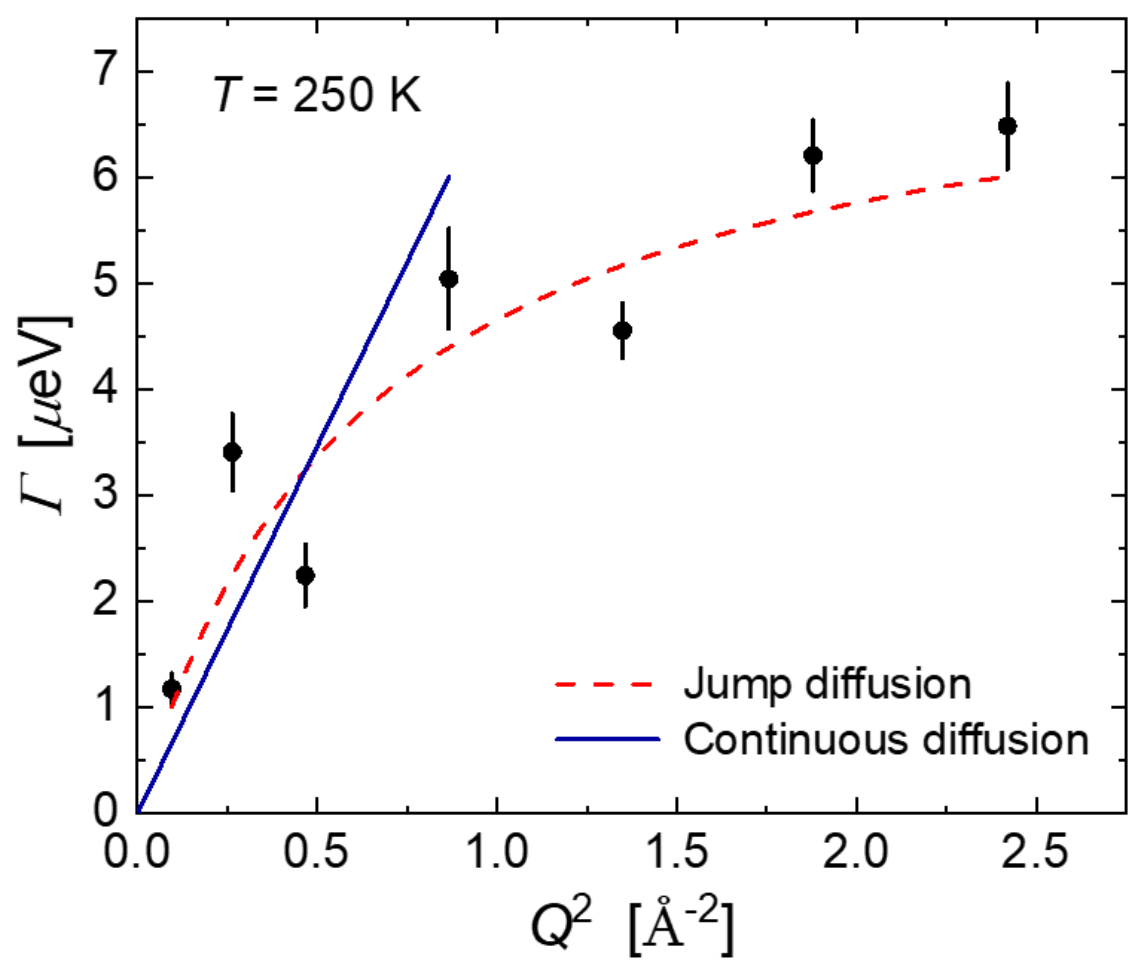

Figure 4.8. HWHM of the single Lorentzian function fit to QENS spectra of the wet CuO sample measured on the HFBS at $250 \mathrm{~K}$. Solid line is a least-squares fit to the low- $Q(<1$ $\AA^{-2}$ ) data, and the dashed curve is a fit of the data to a jump diffusion model [161]. 


\subsubsection{QENS Measured on BaSiS}

Following the preliminary QENS measurement on the HFBS presented in the previous section, we received eight days of beam time on BaSiS at ORNL to perform QENS measurements. Utilizing the larger dynamic range of BaSiS, the goal of these measurements was to investigate the diffusion of water near $\mathrm{CuO}$ nanostructures and to compare it with that of bulk supercooled water [161]. As discussed earlier, our elastic scans (Fig. 4.5) suggested obtaining QENS spectra at $270 \mathrm{~K}$ where our wet and dry samples share a common freezing behavior; at $250 \mathrm{~K}$ where water in the wet sample freezes more rapidly on cooling; and, at $230 \mathrm{~K}$ where most of the water has been immobilized.

The samples measured on BaSiS are identical to those measured on HFBS: 100 , 5-cm diameter $\mathrm{CuO}$-coated copper foils stacked and sealed in an aluminum can with 60 $\mu \mathrm{l}$ ("wet") and $10 \mu \mathrm{l}$ ("dry") of $\mathrm{H}_{2} \mathrm{O}$ under a helium atmosphere (see Appendix A for more details). Samples were cooled from $\sim 300 \mathrm{~K}$ to $275 \mathrm{~K}$ at a temperature ramp rate of $0.2 \mathrm{~K} / \mathrm{min}$ that was reduced to $0.05 \mathrm{~K} / \mathrm{min}$ until reaching $270 \mathrm{~K}$. At this point, QENS spectra were measured for approximately 3 hours at $270 \mathrm{~K}$ and the process repeated at $260 \mathrm{~K}, 250 \mathrm{~K}, 240 \mathrm{~K}$, and $230 \mathrm{~K}$, which span the continuous freezing transition in Fig. 4.5. After measuring QENS at $230 \mathrm{~K}$, the sample-specific resolution function $R(Q, \omega)$ was recorded at $100 \mathrm{~K}$ where the diffusive motions of the $\mathrm{H}$ atoms in the water molecules are frozen out and only the elastic signal is measured. 
All spectra were reduced using standard tools available at ORNL and fit using the software DAVE [159]. The detectors on BaSiS were normalized using a vanadium powder standard at $\sim 6 \mathrm{~K}$ in a sample cell identical to those of our samples. Using DAVE, Eq. (4.3) was fit to all $Q$ values simultaneously where the only constraint was to fix the centers of the delta and Lorentzian components. The other fitting parameters were adjusted and/or constrained before being finally relaxed in order to confirm the overall fits (red curve in Fig. 4.9), which represent the global minima in the LevenbergMarquardt least-squares fitting algorithm used in DAVE. Attempts to fit spectra with more than two Lorentzians gave either unphysical results or fits that depended on the initial set of parameters. The motions represented by the narrow Lorentzian are slower than those represented by the broad Lorentzian.

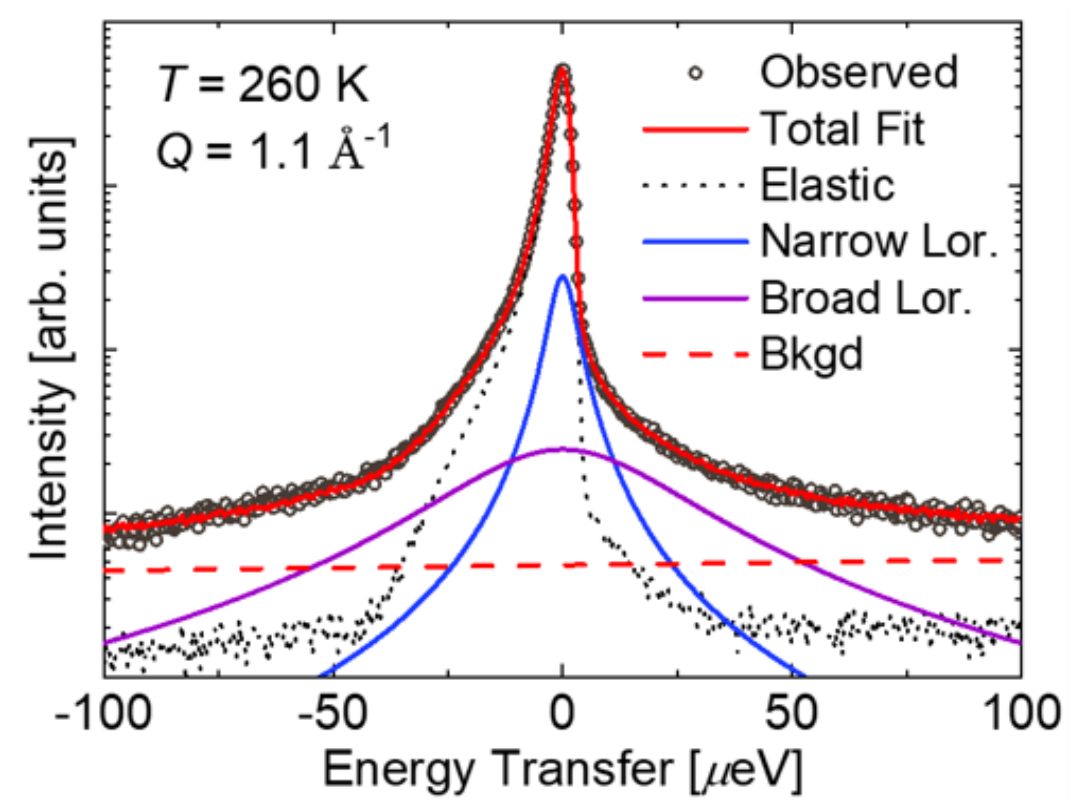

Figure 4.9. QENS spectrum of the wet CuO-coated sample measured on $\mathrm{BaSiS}$ at $Q=1.1$ $\AA^{-1}$ after cooling to $260 \mathrm{~K}$. The data points (open circles) were fit by folding the instrument resolution function with a scattering law consisting of three terms: elastic scattering described by a delta function (black dotted curve) and a broad Lorentzian (magenta curve) plus a narrow Lorentzian (blue curve) that describe the quasielastic scattering. The best fit to the spectrum (red curve) is obtained after adding a linear background term (dashed red line). 


\subsubsection{Comparing HFBS and BaSiS Elastic Intensities}

Because the HFBS and BaSiS have similar configurations and energy resolution $(\sim 1 \mu \mathrm{eV}$ vs. $\sim 3.5 \mu \mathrm{eV}$, respectively), it is desirable to check our sample reproducibility by comparing the temperature dependence of the elastic intensity measured on the two spectrometers. In Fig. 4.10, the HFBS elastic scans have been reproduced from Fig. 3.3 for our two $\mathrm{CuO}$ samples along with the intensity $A(Q)$ of the delta function component (black points) in the $\mathrm{BaSiS}$ spectra summed over the $Q$ range of interest. To make this comparison, we treated the data in the following way. First, the elastic intensities were integrated over similar $Q$ ranges $\left(0.36 \AA^{-1}<Q<1.75 \AA^{-1}\right.$ for HFBS and $0.30 \AA^{-1}<Q<$ $1.70 \AA^{-1}$ for BaSiS). Then, a scale factor was applied to the data of the wet sample such that its $Q$-integrated elastic intensity is equal to that of the dry sample at a temperature of $270 \mathrm{~K}$. This scaling accounts for an unequal amount of copper in the beam. The temperature of $270 \mathrm{~K}$ was selected because the samples contained different amounts of water, and at $270 \mathrm{~K}$ the amount of frozen/immobile that could contribute to the elastic intensity is small compared to that at $200 \mathrm{~K}$. Finally, the HFBS elastic intensities were scaled by a multiplicative factor that accounts for differences in the throughput of the two spectrometers.

With this normalization scheme, the relative change in the $Q$-integrated elastic intensities measured on the two spectrometers agree remarkably well in Fig. 4.10. However, further analysis is required to validate the use of this single scale factor to correct for difference in spectrometer configuration and background. The slight differences between the elastic intensities might be related to the coarser energy resolution of BaSiS $(3.5 \mu \mathrm{eV})$ compared to HFBS $(1 \mu \mathrm{eV})$ where quasielastic scattering 
near the elastic line (e.g., between 1 and $3.5 \mu \mathrm{eV}$ ) on the HFBS would be resolutionlimited on BaSiS. Another source of error in this comparison could be related to the difference in measurement time. The QENS spectra were recorded over a time span of 24 hours on BaSiS vs. several minutes on HFBS. Given the fewer temperature points measured on BaSiS, we cannot confirm the presence of two different freezing regimes in the wet sample as observed in the FWS measured on the HFBS (Fig. 3.3). Nevertheless, we conclude that the slope of the elastic intensity of the wet $\mathrm{CuO}$ sample is greater than that of the dry sample over the temperature range $230-260 \mathrm{~K}$ as measured on both spectrometers.

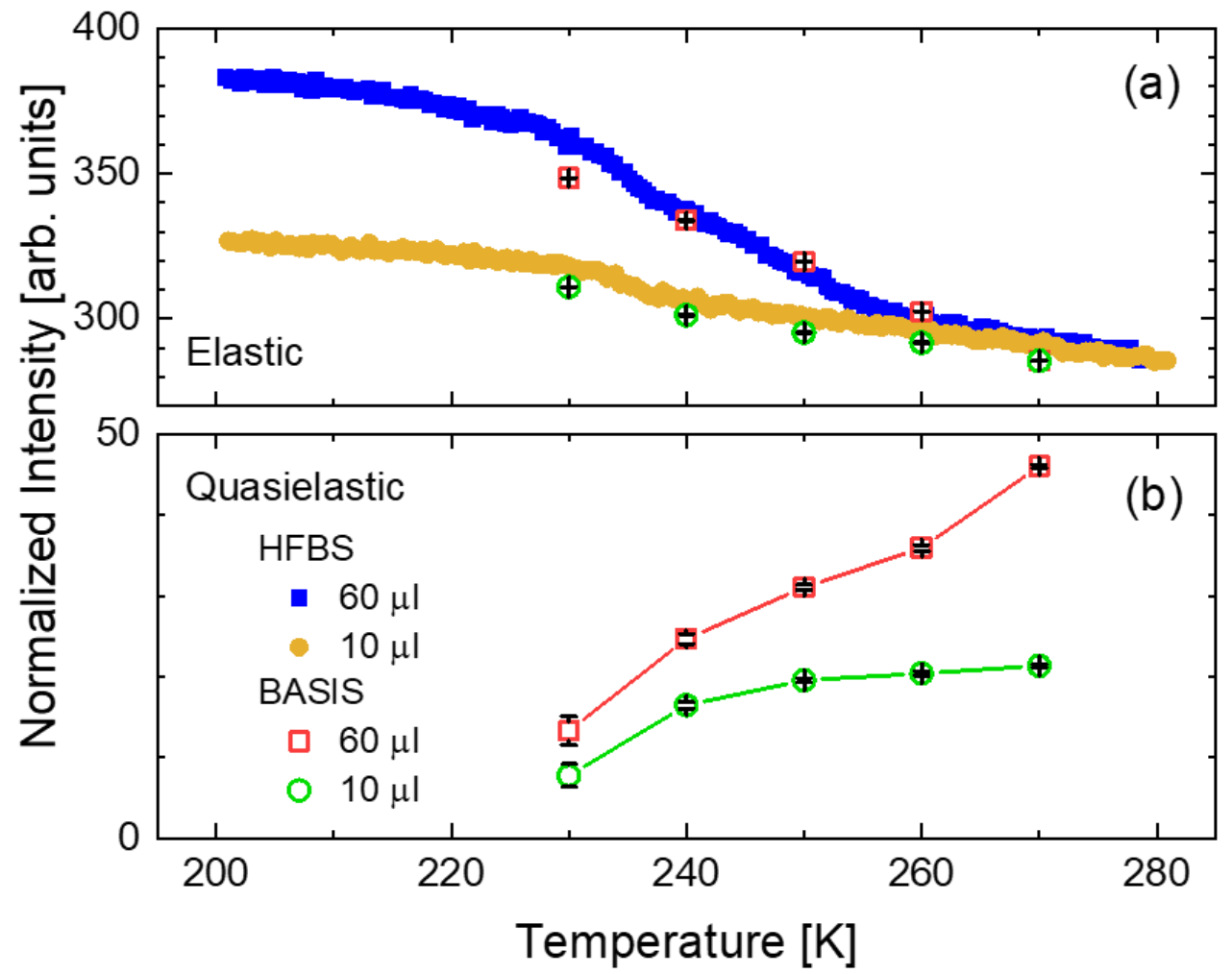

Figure 4.10. (a) Elastic intensity vs. temperature measured on HFBS (blue and orange points) and $\mathrm{BaSiS}$ (red and green points) for the wet $\mathrm{CuO}$ (squares) and dry (circles) $\mathrm{CuO}$-coated samples. (b) Integrated intensities of the quasielastic components (narrow plus broad Lorentzians) measured on $\mathrm{BaSiS}$ as a function of temperature. See text for details on scaling. The black lines within the data points are error bars that represent one standard deviation in the uncertainty in the measured intensity. 
The temperature dependence of the quasielastic intensity of each sample is shown in Fig. 4.10b as a sum of the $Q$-integrated intensities of the narrow and broad Lorentzians measured on BaSiS. At all temperatures, the quasielastic intensity of the wet sample is greater than that of the dry sample by a factor of $\sim 2.2$ difference at $270 \mathrm{~K}$ that decreases to $\sim 1.6$ at $230 \mathrm{~K}$. The gradual loss of quasielastic intensity combined with a gradual gain in elastic intensity over this temperature range indicates that the water is freezing continuously on a nanosecond timescale, consistent with our analysis of continuous freezing of water from our HFBS fixed-window scans. Moreover, the greater slope in the data of the wet sample indicates that continuous freezing occurs at a faster rate than the dry sample, similar to the greater slope of the second linear term in the HFBS scans.

We note that there is a discrepancy between the relative change in elastic intensity vs. quasielastic intensity for the two samples in Fig. 4.10. Specifically, the elastic intensity of the wet (dry) sample increased by about 60 (25) arbitrary units whereas the quasielastic intensity decreased by about 30 (13) arb. units on cooling from 270 to $230 \mathrm{~K}$. The origin of this factor of two difference between these intensity changes for both the wet and dry samples is currently unknown; however, the most likely cause is quasielastic scattering that was too broad to be measured within the dynamic range of $\mathrm{BaSiS}$. As the sample is cooled, these modes freeze out and contribute to the larger (factor of two) increase of the elastic intensity. Support for this possibility is provided by the temperature dependence of the background term fitted to the QENS spectra, which is plotted in Fig. 4.11. The gradual decrease in the background intensity, equal in magnitude for both samples, could indicate that quasielastic scattering is entering the dynamic range of $\mathrm{BaSiS}$ as the samples are cooled from $270 \mathrm{~K}$ to $230 \mathrm{~K}$. 


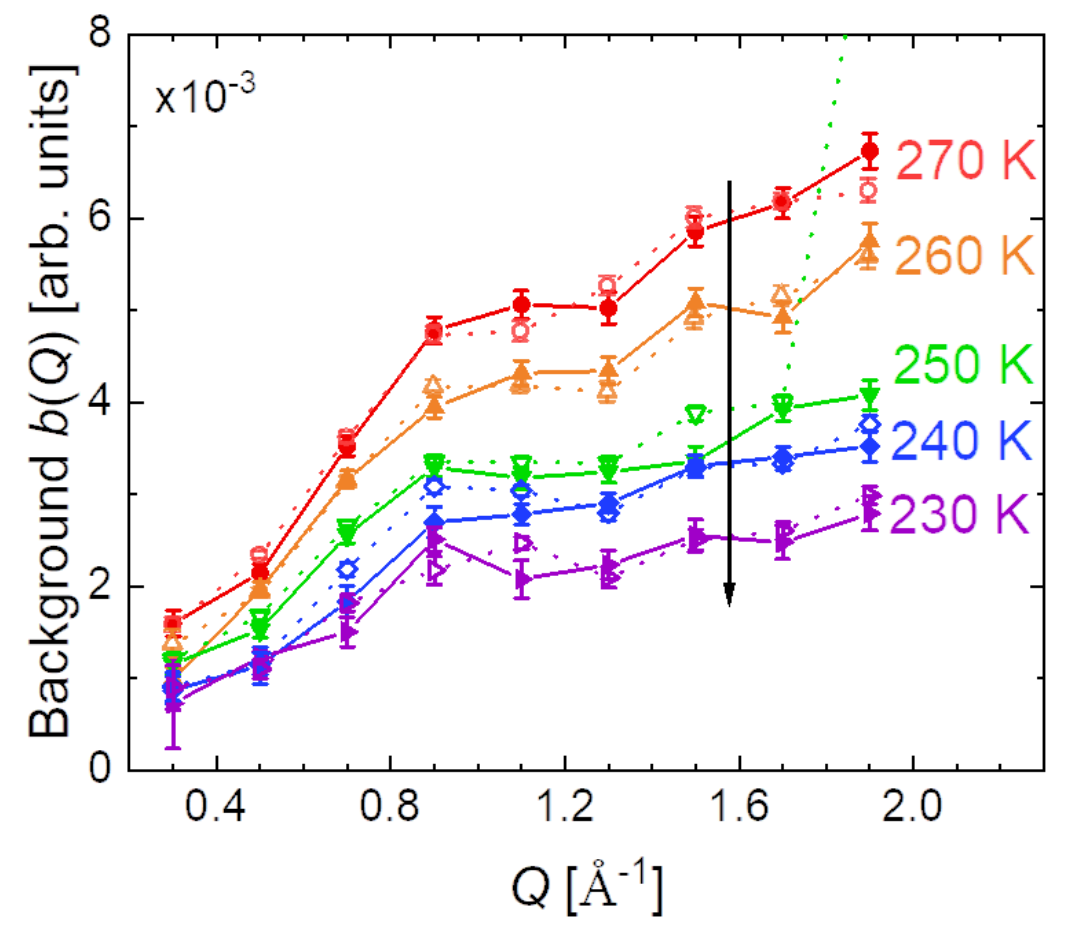

Figure 4.11. Intensity of the background term $b(Q)$ in the linear background intensity $V(Q, \omega)=a(Q) \omega+b(Q)$ plotted versus $Q$ for the wet (closed points) and dry (open points) samples at all temperatures measured on BaSiS. (Outlying data point at $250 \mathrm{~K}$ is at $\sim 10^{-2}$ arb. units). Lines are drawn as guides to the eye. The arrow indicates that the background intensities of both samples decreased as the samples were cooled from $270 \mathrm{~K}$ to $230 \mathrm{~K}$.

\subsubsection{Motions Occurring on Two Time Scales}

As noted above, the larger dynamic range of BaSiS compared to HFBS allowed us to resolve two distinct diffusive motions of the $\mathrm{H}$ atoms in water: "slow" and "fast" motions described by narrow and broad Lorentzians, respectively. A similar analysis of interfacial water near single-supported bilayer lipid membranes allowed us to identify the broad Lorentzian as representing the diffusion of bulk-like water that is different from water that interacts strongly with melittin peptides represented by a narrow Lorentzian [158]. For both $\mathrm{CuO}$ samples, the temperature dependence of the intensity of the narrow and broad Lorentzians is plotted individually in Fig. $\mathbf{4 . 1 2}$ in order to provide insight into 
the continuous freezing transition of water near the $\mathrm{CuO}$ nanostructures. As with the intensities plotted in Fig. 4.10b, those in Fig. 4.12 have been summed over the $Q$ range 0.3-1.7 $\AA^{-1}$ similar to that of the HFBS. For all temperatures, we see that the intensity of the narrow component exceeds that of the broad component. Furthermore, as the water content of the sample increases, the intensities of both the narrow and broad components increase as well. That is, both samples contain more "slowly" rather than "rapidly" diffusing water, and the amount of water moving on each time scale increases as the hydration level of the sample increases.

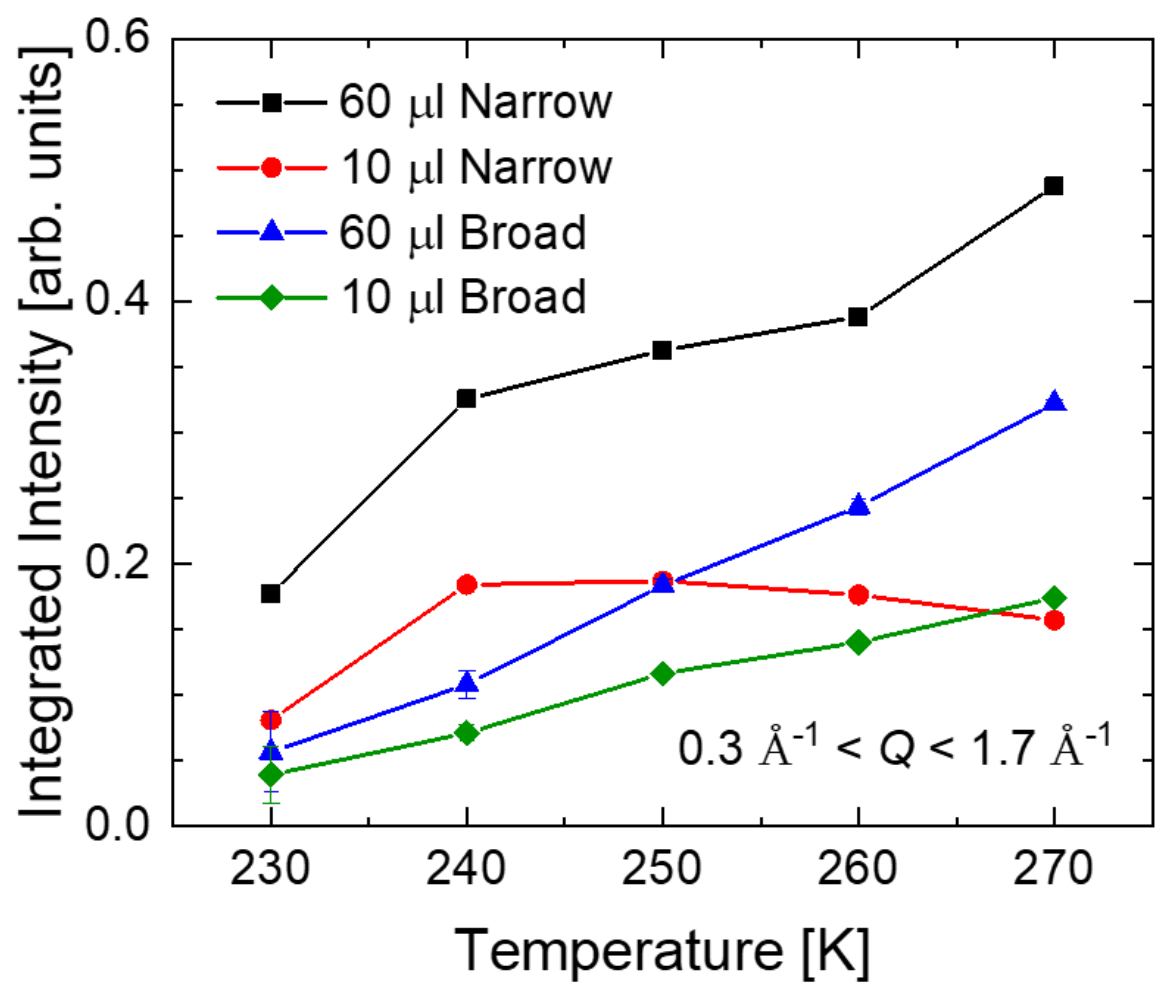

Figure 4.12. Temperature dependence of the intensity of the narrow and broad Lorentzians in the BaSiS spectra of the wet and dry $\mathrm{CuO}$-coated samples integrated over the $Q$-range 0.3-1.7 $\AA^{-1}$. To account for the possibility of a different number of foils in the scattering volume of the two samples, the spectra of the wet sample have been scaled so that the intensity of the delta function summed over all $Q$ at $270 \mathrm{~K}$ agrees with that of the dry sample (the delta function intensity is dominated by elastic scattering of the foils at $270 \mathrm{~K}$ ). Error bars representing a statistical uncertainty of one standard deviation are smaller than the size of the data points. 


\subsubsection{The Broad Lorentzian}

To probe the type of motion (e.g., translational or localized/rotational) occurring on the two different timescales, we examined the $Q$ dependence of the HWHM of the two Lorentzians. In Fig. 4.13, the HWHM of the broad Lorentzian is plotted vs. $Q^{2}$ at several temperatures for the wet and dry samples (for clarity, the data at $240 \mathrm{~K}$ and $260 \mathrm{~K}$ are not shown). Also plotted are data for bulk supercooled water measured at $253 \mathrm{~K}$ from Ref. [161]. In the temperature range 240-270 K, the HWHM for both samples exhibit very little temperature dependence and follow the HWHM of bulk supercooled water at low $Q$. These features suggest that the water represented by the broad Lorentzian is similar to bulk supercooled water undergoing translational diffusion at $253 \mathrm{~K}$ [161]. The deviation of $\Gamma_{B}$ from that of bulk water at a lower $Q$ seen for the dry sample compared to the wet sample (about 1 and $2 \AA^{-2}$, respectively) may result from a smaller amount of bulk-like water in the dry sample (consistent with the weaker intensity of the broad Lorentzian in the dry sample in Fig. 4.12). The more distorted shape and lower intensity of the librational band of the dry sample seen in our vibrational spectra in Fig. 4.4 also support the dry sample containing less bulk-like water than the wet sample. 

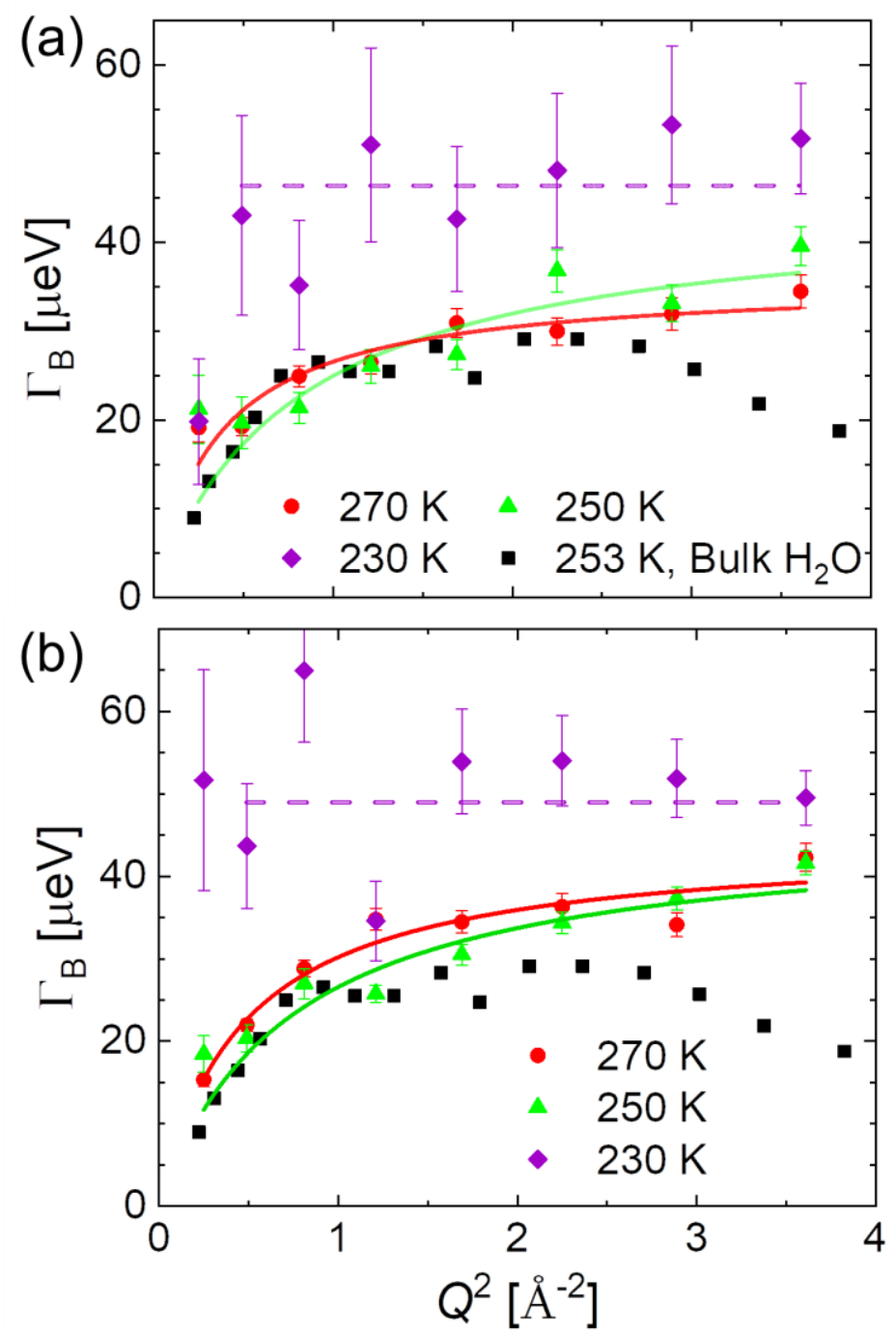

Figure 4.13. HWHM vs $Q^{2}$ of the broad Lorentzian as measured on BaSiS at 270, 250, and $230 \mathrm{~K}$. Data for bulk water are from Ref. [161]. Lines are drawn as guides to the eye.

The similarity between $\Gamma_{B}$ of the wet and dry samples at low- $Q$ with that of bulk supercooled water in Fig. 4.13 in the temperature range 240-270 K supports the presence of bulk-like water in both samples albeit there is less in the dry sample. Therefore, we infer that the freezing of bulk-like water is contributing to both linear terms (red and green data points) in the HFBS elastic scans in Fig. 4.5-a conclusion that could not have been reached from the elastic intensity scans alone. Presumably, this bulk-like water 
represents water molecules undergoing translation diffusion in a location further from the $\mathrm{CuO}$ surface and which interacts relatively weakly with the nanostructures.

Although the dynamics of the interfacial water in our samples represented by the broad Lorentzian appears similar to bulk supercooled water at a temperature of $\sim 250 \mathrm{~K}$ (Fig. 4.13), we note that the elastic intensity scan (Fig. 4.5) shows no evidence of a discontinuity or an abrupt intensity change as was observed for water in proximity to bilayer lipid membranes $[\mathbf{1 2 9}, \mathbf{1 5 8}]$. Furthermore, the abrupt change in elastic intensity observed in the lipid system occurred at the same temperature at which Bragg peaks of hexagonal ice appeared [137]. In the $\mathrm{CuO}$ system, however, even with a sample containing $120 \mu \mathrm{l}$ of $\mathrm{D}_{2} \mathrm{O}$, i.e., twice the amount of $\mathrm{H}_{2} \mathrm{O}$ as the wet sample reported here, we have not observed Bragg peaks of crystalline ice in repeated scans (see Fig. 3.7 and related discussion). These results reinforce our use of the term "bulk-like" in referring to the presence of water with dynamics similar to that of bulk supercooled water.

We also note that the HWHM of bulk supercooled water can differ slightly depending on the experimental conditions (e.g., instrument energy resolution and temperature). For example, the HWHM of the broad Lorentzian of the wet sample is plotted in Fig. 4.14 juxtaposed with the HWHM measured for bulk supercooled water at about the same temperature $(260 \mathrm{~K})$ by various other research groups: Di Cola et al. [162], J. Qvist et al. [163], J. Teixeira et al. [161], and F. Cavatorta et al. [164]. At high $Q$, the HWHM measured by the other investigators decreases monotonically as the temperature is reduced. Also, the data of our sample may be affected by the smaller dynamic range of $\mathrm{BaSiS}( \pm 120 \mu \mathrm{eV})$ compared to the spectrometers used by the other investigators. 


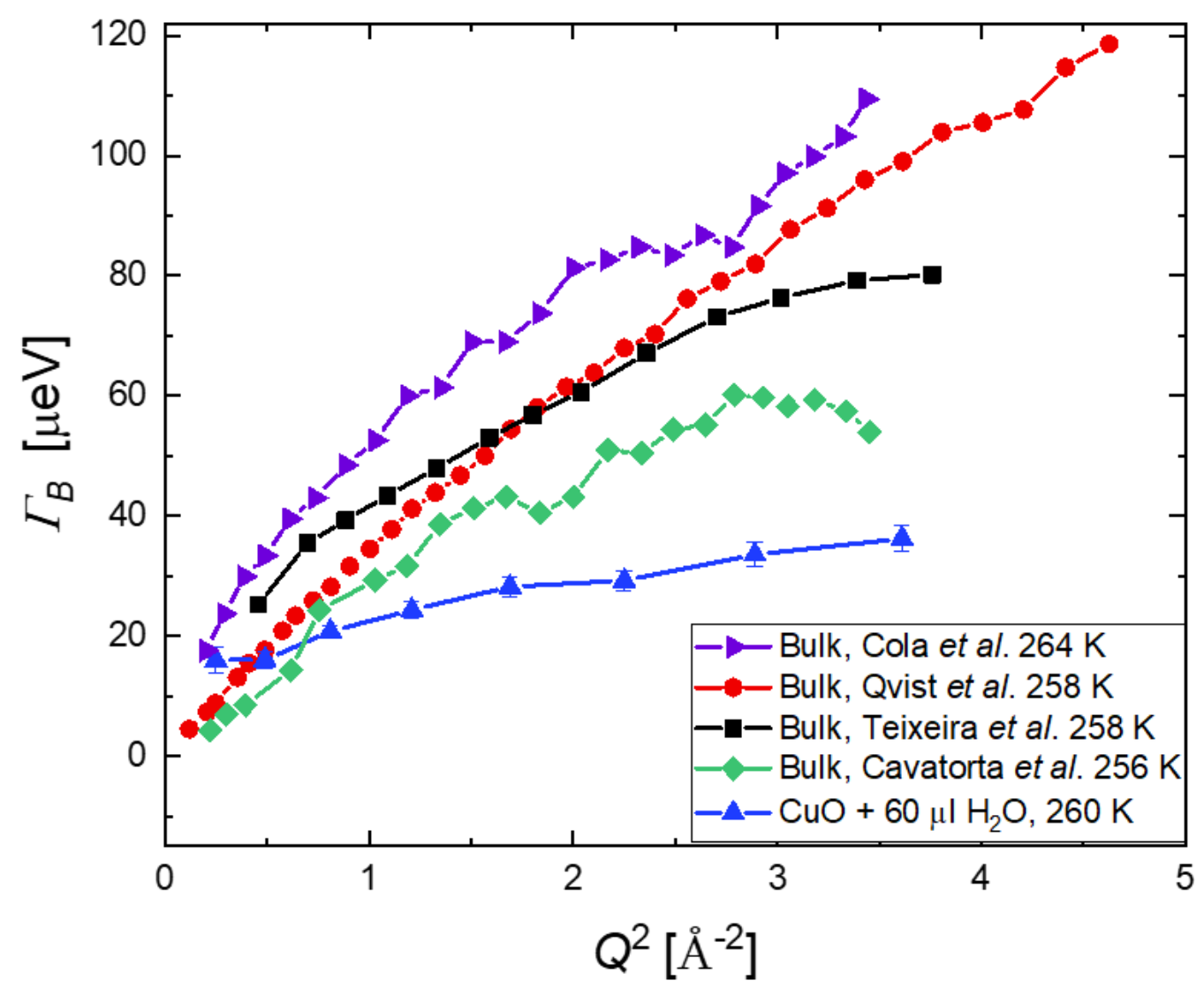

Figure 4.14. HWHM of Lorentzian functions fit to QENS spectra of supercooled bulk water at a temperature around $260 \mathrm{~K}$ by various research groups: Di Cola et al. [162] (purple right-arrows), Qvist et al. [163] (red circles), Teixeira et al. [161] (black squares), Cavatorta et al. [164] (green diamonds), and this work (blue triangles).

As shown in Fig. 4.13, we see that a qualitative change in the $Q$-dependence of $\Gamma_{B}$ occurs on reducing the temperature from $240 \mathrm{~K}$ to $230 \mathrm{~K}$. The magnitude of $\Gamma_{B}$ increases and becomes nearly $Q$ independent at $230 \mathrm{~K}$, which is inconsistent with bulk supercooled water undergoing translational diffusion [161]. The magnitude of $\Gamma_{B}$ of the wet (dry) sample fluctuates around a value of $\sim 46.3(48.9) \mu \mathrm{eV}$ as determined by a fit to a constant over the range $Q>0.7 \AA^{-1}$, which corresponds to a relaxation rate occurring on the $\sim 89$ $(\sim 85)$ ps time scale. We interpret the temperature dependence observed for $\Gamma_{B}$ as indicating that there are actually two types of diffusive motion occurring at a comparable rate that are unresolved and contributing to the broad Lorentzian at higher temperatures: 
(1) translational diffusion of bulk-like water and (2) water molecules undergoing rotational diffusion that contributes a $Q$-independent component to the width of the quasielastic peak [160]. As the sample temperature decreases below $240 \mathrm{~K}$, the translational diffusive motion of the bulk-like water freezes out so that by $230 \mathrm{~K}$ only the rotational motion contributes to the broad Lorentzian.

The question remains as to the location of the rotational diffusive motion at $230 \mathrm{~K}$ observed in Fig. 4.13. As the rotational motion survives the freezing-out of bulk-like water below $240 \mathrm{~K}$, it evidently is not strongly coupled to the translational motion of bulk-like water. To explore this possibility further, it is of interest to study the temperature dependence of the translational and rotational motions that contribute to the broad Lorentzian. Although we have been unable to decompose the broad Lorentzian into two separate components by introducing a third Lorentzian, we can probe the temperature dependence of broad Lorentzian more indirectly. We begin by noting that the intensity of quasielastic scattering from a water molecule rotating either about its center of mass or a symmetry axis will be a minimum at $Q=0$ and increase monotonically in the $Q$ range of our experiments. In contrast, the quasielastic intensity should be independent of $Q$ for center-of-mass translations $[\mathbf{8 0 , 1 6 0}]$.

Plotted in Fig. 4.15a is the intensity of the broad Lorentzian for both the wet and dry $\mathrm{CuO}$ samples which has been summed over two different $Q$ ranges: low $Q\left(0.3 \AA^{-1}<\right.$ $\left.Q<0.9 \AA^{-1}\right)$ and high $Q\left(1.1 \AA^{-1}<Q<1.7 \AA^{-1}\right)$. For the reason explained above, the sums over the low- $Q$ and high- $Q$ ranges will weight the broad Lorentzian intensity toward the translational and rotational diffusion components, respectively. Accordingly, in Fig. 4.15a the intensity of the broad Lorentzian, summed over the high- $Q$ range, decreases 
more slowly than that over the low- $Q$ range on cooling. This difference supports our interpretation that both bulk-like translation motion and rotational diffusion are contributing to the broad Lorentzian's intensity. Moreover, we infer that the contribution to the broad Lorentzian intensity from water diffusing rotationally has a weaker temperature dependence than that of the water undergoing translational motion $-\mathrm{a}$ feature that is more pronounced in the dry sample. Because the dry sample has less bulklike water, we are led to the conclusion that the rotational diffusive motion that we are observing is more likely occurring in water that is in close proximity to the nanostructures. 


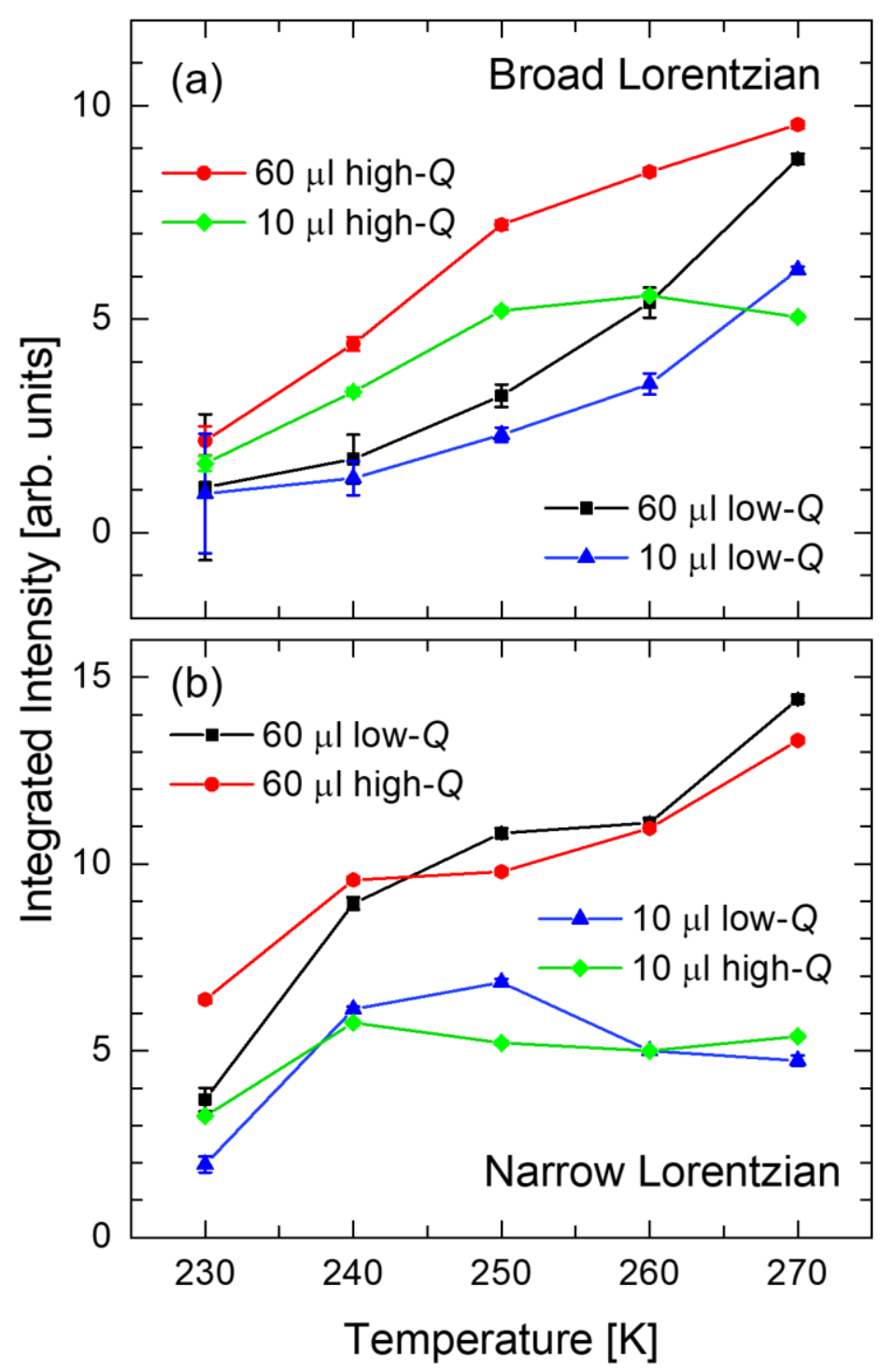

Figure 4.15. Temperature dependence of the $Q$-weighted intensity of the (a) broad and (b) narrow Lorentzian components in the BaSiS spectra of both the wet and dry CuO-coated samples. The intensity of each Lorentzian has been summed over two different $Q$ ranges: low $Q\left(0.3-0.9 \AA^{-1}\right)$ and high $Q\left(1.1-1.7 \AA^{-1}\right)$. As in Fig. 4.12, the Lorentzian intensities have been scaled to allow for a different number of foils in the scattering volume.

As discussed in Sec. 1.3.2., a variety of other systems containing confined water have shown evidence of rotational diffusive motion at low temperatures. These studies include supercooled water in Vycor [80] (porous silica glass) and water at protein interfaces [165]. As in the case of our CuO-coated samples, no Bragg peaks indicative of crystallization was observed for a water $\left(\mathrm{D}_{2} \mathrm{O}\right)$ monolayer adsorbed on the pore walls of 
Vycor [80]. The elastic scans by Zanotti et al. [80], with $1 \mu \mathrm{eV}$ energy resolution, revealed both translational and rotational components that contribute to the mean-squared displacements as indicated by inflection points occurring at $240 \mathrm{~K}$ (translational) and 220 $\mathrm{K}$ (localized rotational). These results appear consistent with our observation of freezing out of a "fast" translational motion between $240 \mathrm{~K}$ and $230 \mathrm{~K}$ prior to observation of the rotational motion.

The structure, freezing behavior, and dynamics of water confined to mesoporous silicas have been investigated in some detail $[\mathbf{9 4 , 1 2 6 , 1 3 1 , 1 6 6}]$. Neutron diffraction measurements have provided evidence of water in a glassy state lining the pore walls in a layer three molecules thick at $240 \mathrm{~K}[\mathbf{1 2 6}]$. However, unlike the water in our samples, this state converts reversibly to a defective form of ice (hexagonal and cubic containing stacking faults) at lower temperatures. NMR relaxation studies below $260 \mathrm{~K}$ on ice in several different forms of silica have shown that the disordered ice can be characterized as having proton mobility intermediate between that of bulk water and crystalline ice. It is also evident that the motion of the disordered ice is predominantly rotational rather than translational $[\mathbf{9 4 , 1 2 6}]$. The enhanced mobility over that in the crystalline ice phase was attributed to changes in the rotational freedom arising from the creation of a more hydrogen-bonded local environment. This disordered interfacial water was termed a "plastic crystal" $[\mathbf{9 4 , 1 2 6}]$ in analogy with other crystals that have well-defined lattice structures but have molecules that are orientationally disordered [167]. 


\subsubsection{The Narrow Lorentzian}

We next turn to the narrow Lorentzian component in the QENS spectra, which is contributed by water molecules undergoing diffusive motion an order of magnitude slower than those contributing to the broad Lorentzian. The HWHM of the narrow Lorentzian $\Gamma_{N}$ of the wet and dry samples is plotted in Fig. 4.16 as a function of $Q^{2}$ at several temperatures. As was the case for the broad Lorentzian, the wet and dry samples yield similar results. For $Q^{2} \geq 1.5 \AA^{-2}, \Gamma_{N}$ of both samples approaches a constant value above $240 \mathrm{~K}$. This behavior is characteristic of jump diffusion models used to interpret the translational dynamics of bulk supercooled water [161]. However, besides the relatively small value of $\Gamma_{N}$, the results in Fig. 4.16 differ qualitatively from bulk supercooled water in two respects. First, for both samples, $\Gamma_{N}$ increases monotonically from $\sim 3.5$ to $\sim 4.5 \mu \mathrm{eV}$ rather than decrease on cooling from $270 \mathrm{~K}$ to $240 \mathrm{~K}$ [161]. Secondly, at the lowest temperature of $230 \mathrm{~K}$, the magnitude of $\Gamma_{N}$ abruptly decreases at low $Q$ and fails to saturate at high $Q$. These anomalies suggest that the narrow Lorentzian is contributed by water that interacts strongly with and hence closest to the $\mathrm{CuO}$ nanostructures. 

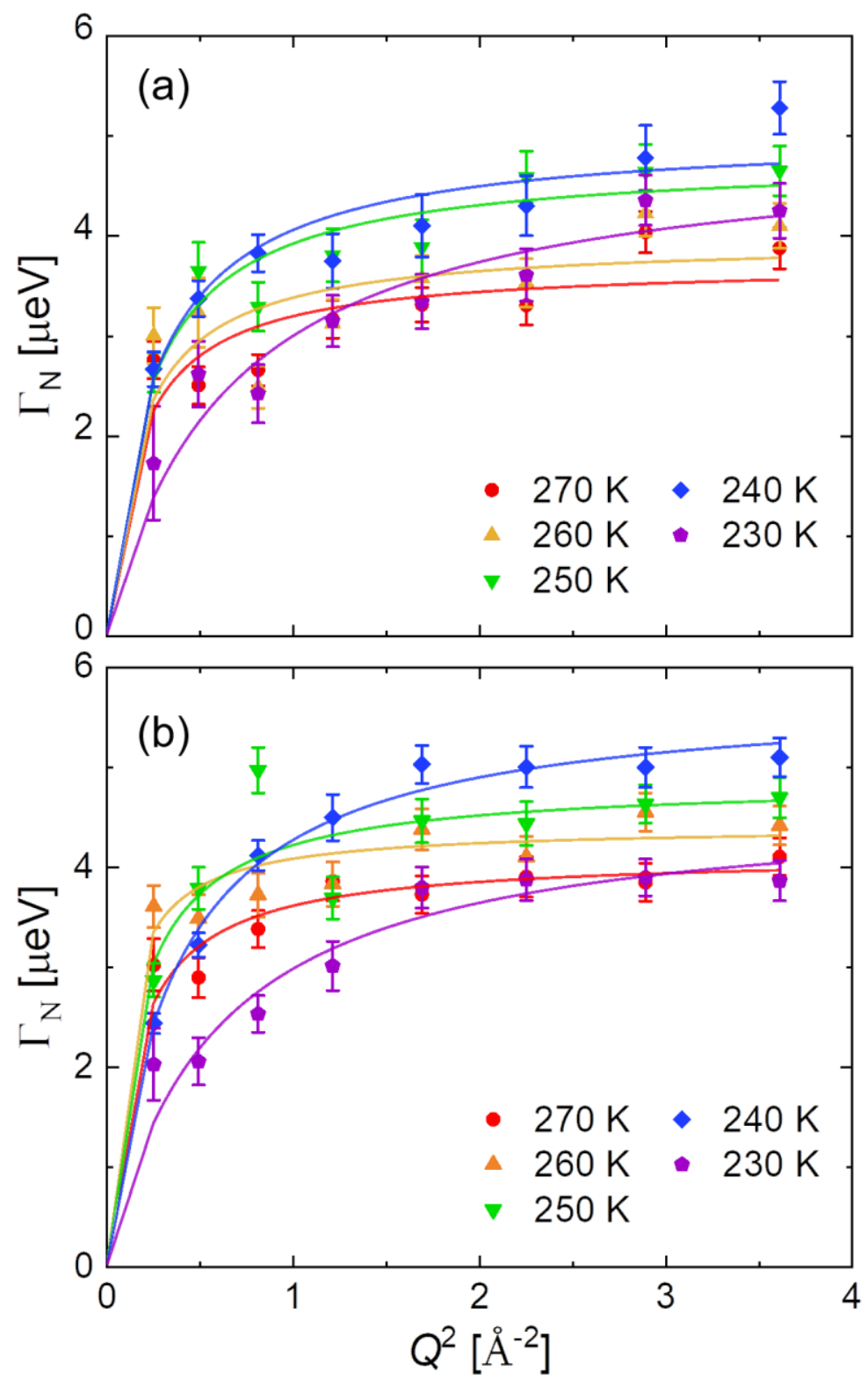

Figure 4.16. HWHM of the narrow Lorentzian $\Gamma_{N}$ as a function of $Q^{2}$ at selected temperatures as measured on BaSiS for (a) the wet $(60 \mu \mathrm{l})$ and (b) dry $(10 \mu \mathrm{l}) \mathrm{CuO}$ coated samples. Solid curves are guides to the eye.

To determine the location of the slowly diffusing water represented by the narrow Lorentzian, we start by considering the temperature dependence of the narrow Lorentzian intensity of the dry sample since the broad Lorentzian intensity of the broad sample is weaker than that of the wet sample (see Fig. 4.12). We see in Fig. 4.12 that the intensity of the narrow Lorentzian has very little temperature dependence down to $240 \mathrm{~K}$. This 
feature is similar to that observed for highly confined water, for example, near the anionic head groups of bilayer lipid membranes [129]. Likewise, we propose that the narrow Lorentzian component of the dry sample arises due to more slowly diffusing water performing a bounded motion close to the nanostructures. From the similarity in the width of the narrow Lorentzian component in both the dry and wet samples (Fig. 4.16) as well as the common initial slope in their elastic scans (Fig. 4.5), we suggest that this interpretation also applies to the wet sample.

In considering the type of diffusive motion (e.g., translational or rotational) represented by the narrow Lorentzian, we see in Fig. 4.16 that, like the broad Lorentzian, $\Gamma_{N}$ generally increases with $Q$ at low $Q$. The quality of these data, however, does not allow a determination of whether Fick's law of translational diffusion is obeyed. Instead, as was the case for the broad Lorentzian, it is useful to compare the low- $Q$ and high- $Q$ behavior of the narrow Lorentzian intensity. In Fig. $\mathbf{4 . 1 5 b}$, we see that, for both samples, there is essentially no difference between the temperature dependence of the narrow Lorentzian intensity at low and high $Q$. This behavior differs from that of the broad Lorentzian intensity (Fig. 4.15a) for which a large difference was explained by the presence of rotational diffusion. As discussed above, the intensity of the quasielastic scattering from a sample undergoing translational diffusion should be $Q$-independent [160]. Thus, the absence of $Q$ dependence in the temperature dependence of the narrow Lorentzian intensity provides indirect evidence of a relatively slow diffusive motion that is translational in nature.

As discussed in Sec. 3.4, the background levels in our neutron diffraction scans (see, e.g., Fig. C.4) and elastic scans (Fig. 4.5) as well as the strong elastic peak of the 
QENS spectra (Figs. 4.6 and 4.9) and the vibrational spectra of a dehydrated $\mathrm{CuO}$ sample (Fig. 4.3) all provide evidence of immobile water that is present on the $\mathrm{CuO}$ surfaces after annealing a sample at a temperature of $328 \mathrm{~K}$. In fact, this residual water is believed to be the reason why the amount of water in the scattering volume as determined by the FWS of the wet and dry samples differs by only a factor of two and, in both cases, exceed that in the initial droplet $(60 \mu 1$ and $10 \mu 1$, respectively). Such strongly bound water apparently seeds a hydrogen-bonded network that frustrates the crystallization of bulk ice, as indicated by the distorted vibrational spectra of our wet and dry samples in Fig. 4.4 compared to that of bulk ice. Water in the process of forming this nascent hydrogenbonded network may undergo relatively slow translational diffusion represented by the narrow Lorentzian component in our QENS spectra.

The question remains as to why there is a slope change in the elastic scan of the wet sample (green points in Fig. 4.5). As discussed previously in Sec. 3.3, we proposed that the larger slope represents freezing of a second population of water located further from the nanostructures than the strongly bound water represented by the initial slope in the elastic intensity scan (red points in Fig. 4.5). We can now use our QENS results to investigate this question further. As shown in Figs. 4.12 and 4.15, the intensity of the narrow Lorentzian component exceeds that of the broad Lorentzian in each sample. Also, the narrow Lorentzian intensity increases on going from the dry to the wet sample. Assuming that the water-CuO interaction is stronger than the water-water interaction, additional water should preferentially wet along the length of a $\mathrm{CuO}$ blade as shown by ESEM imaging (Fig. 2.11), rather than form a film of uniform thickness as considered previously [168]. Furthermore, if we assume that the contributions to the elastic intensity 
of the wet sample from the two linear terms are additive and that both extend down to a temperature of $\sim 237 \mathrm{~K}$, the second term (green points) contributes about the same number of water molecules as the first term (red points). From QENS, we find that at 270 $\mathrm{K}$ - the temperature at which the water dynamics lies within the dynamic range of BaSiS - the intensity of the narrow Lorentzian of the wet sample is about three times higher than that of the dry sample. On cooling to $230 \mathrm{~K}$, the narrow Lorentzian intensity decreases by a factor of 2.5. By comparison, the intensity of the broad Lorentzian of the wet sample is only about $70 \%$ higher than that of the dry sample at $270 \mathrm{~K}$. Therefore, we tentatively identify the water represented by the narrow Lorentzian as contributing to the second linear term in the elastic intensity on cooling of the wet sample.

We can only speculate as to the origin of the small but reproducible increase in the magnitude of $\Gamma_{N}$ at large $Q$ with decreasing temperature, indicating faster translational diffusion (Figs. 4.6a and 4.7b). As the hydrogen-bond network in the water closest to the nanostructures forms on cooling, the water density may decrease thereby allowing a slightly faster translational diffusion. The abrupt decrease in the magnitude of $\Gamma_{N}$ and the change in its $Q$-dependence on reducing the temperature from $240 \mathrm{~K}$ to $230 \mathrm{~K}$, features present in both the wet and the dry samples, may possibly indicate a transition to a lowerdensity glassy state characterized by a reduced rate of translational diffusion over most of our $Q$ range. We note that the abrupt change in the width of the narrow Lorentzian component in the QENS spectra occurs in the same temperature interval that the contribution to the broad Lorentzian attributed to the translational diffusion of bulk-like water disappears (see Fig. 4.13). The appearance of the rotational motion at $230 \mathrm{~K}$ in the broad Lorentzian component also coincides with the transition to the proposed low- 
density glassy phase. Further evidence of a phase transition comes from the elastic scans in Fig. 4.5 where both CuO-treated samples also show a small but reproducible upturn in intensity at $\sim 237 \mathrm{~K}$.

A transition between a high-density (e.g., high-density liquid HDL) and a low-density hydrogen-bonded amorphous structure (e.g., low-density liquid LDL) has been proposed previously for confined water in a number of different porous materials at temperatures near $230 \mathrm{~K}$ : Vycor ( 240 K) [80]; lysozyme (220 K) [165]; and mesoporous silica ( 229 $\mathrm{K}$ [166], 225-250 K [145]). In each case, the crystallization of water was suppressed by spatial confinement in small or narrow pores and enabled access into "no man's land" of water's $P$ - $T$ phase diagram (purple region in Fig. 4.17).

Figure 4.17 shows the $P-T$ phase diagram for the liquid and amorphous solid states of water at temperatures and pressures in the ranges $100-300 \mathrm{~K}$ and $0-0.3 \mathrm{GPa}$, respectively [169]. Starting from the bulk melting point $T_{M}$ of $273 \mathrm{~K}$, liquid water can be supercooled down to the temperature of homogeneous nucleation $T_{H}$ of $\sim 231 \mathrm{~K}$. However, if one rapidly cools water at a rate in excess of $10^{6} \mathrm{~K} / \mathrm{s}$ [154], then it can be vitrified into lowdensity amorphous ice (LDA). Upon heating LDA (or any other amorphous ice) above $\sim 150 \mathrm{~K}\left(T_{x}\right)$, it crystallizes primarily into hexagonal ice. The temperatures $T_{H}$ and $T_{x}$ thus mark the upper and lower limits of "no man's land," respectively, in Fig. 4.17. Based on the computational work by Poole et al. $[\mathbf{8 3}]$, later corroborated by experiments $[\mathbf{6 3 , 8 5}]$, the existence of two liquid states (LDL and HDL) could explain the observed first-order transition between LDA and HDA and several other anomalies of supercooled water $[82,84]$. 


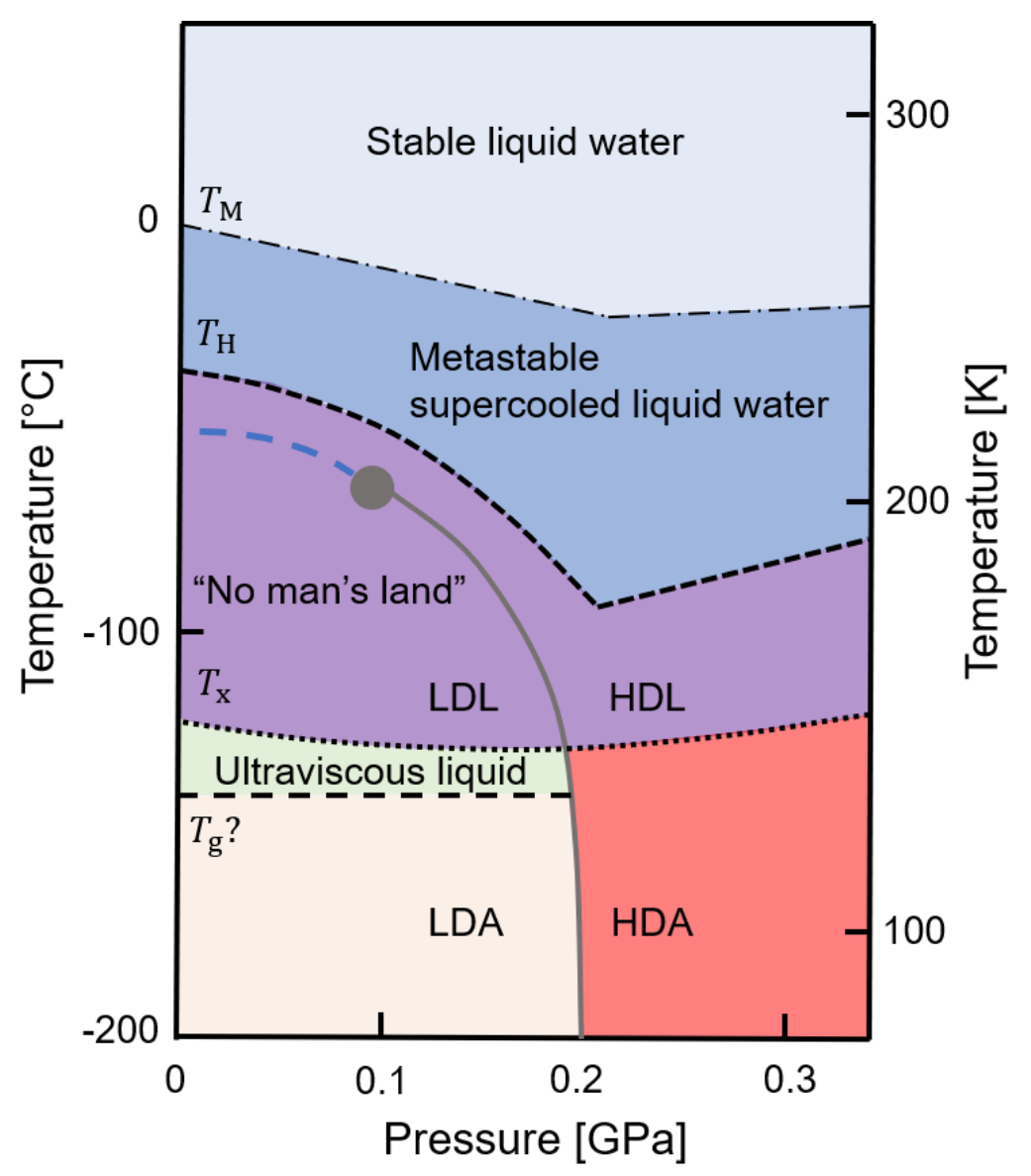

Figure 4.17. Pressure-temperature phase diagram of water adapted from Ref. [169] that depicts a first-order phase transition between low-density (LDL) and high-density liquid (HDL) water. The liquid-liquid coexistence line (grey and blue curves) extends from "no man's land" to below $100 \mathrm{~K}$ that separates amorphous ice phases LDA and HDA.

Confirmation of such a liquid-liquid transition in water in proximity to $\mathrm{CuO}$ nanostructures will require further QENS measurements. The non-cylindrical pore topography associated with the $\mathrm{CuO}$ nanostructures, their exceptional hydrophilicity, and the freezing out of bulk-like supercooled water above the putative transition provide motivation for such studies. 


\section{Chapter 5}

\section{Proposed Future Work}

In this Chapter, we propose a neutron imaging experiment to investigate the thermal performance of water-charged oscillating heat pipes (OHPs) as well as describe a conceptual design of a neutron imaging facility for construction at the University of Missouri Research Reactor (MURR). Specifically, in Sec. 5.1 we propose a neutron imaging experiment for two water-charged, copper flat-plate OHPs (FP-OHPs) with and without a nanostructured-CuO coating [56]. The aims of this experiment are twofold: (1) to compare the thermal performance between bare and $\mathrm{CuO}$-coated OHPs as a function of heat input to the evaporator section; and (2) to measure the amplitude and frequency of water ("slug") oscillations, which are believed to be greater in the CuO-coated FP-OHP compared to a bare OHP. The proposed measurements were contained in a user proposal submitted in October 2018 for neutron beam time to conduct an experiment at the CG-1D Neutron Imaging Facility [170] located at the High-Flux Isotope Reactor (HFIR) at Oak Ridge National Laboratory (ORNL). Although our proposal was accepted, the scheduled beam time was delayed due to unforeseen circumstances at the HFIR [171].

The conceptual design of a thermal neutron imaging facility at MURR is presented in Sec. 5.2 and contains descriptions and locations of critical components, including apertures, filters, gamma and neutron radiation shielding, sample mount, and neutron detector. Principal collaborators on the design of this imaging station are Drs. Kenneth Herwig and Hassina Bilheux of ORNL and Dr. Helmut Kaiser of MURR. 


\subsection{Time-Resolved Neutron Imaging of Liquid Water Dynamics in a CuO-Coated Copper FP-OHP}

\subsubsection{Background}

OHPs offer a promising heat transfer device for a variety of applications, including the cooling of electronic devices [11]. An OHP (see, e.g., Fig. 1.3a) consists of a narrow channel meandering between a heat source (evaporator) and heat sink (condenser) that can be made of tubing with a small inner diameter (T-OHP) or fabricated from a flat-plate with a machined groove (FP-OHP). The OHP is sealed, evacuated, and partially backfilled with a working fluid such as water. The diameter of the channel is sufficiently small such that the fluid forms a chain of alternating liquid "slugs" and vapor "bubbles." Heat addition and rejection at the evaporator and condenser, respectively, cause vapor pressure fluctuations within the channel and drive an oscillating motion of the slugs and bubbles thereby enabling both sensible and latent heat transfer.

Previously, OHP thermal performance has been measured along with characterization of the fluid oscillations by high-speed photography. The latter requires all or part of the OHP to be constructed from optically transparent materials like glass or plastic [26], which have inherently low thermal conductivities. To overcome this limitation, neutron radiography has been used $[\mathbf{1 6 , 1 7 2}]$ to visualize liquid water flow inside copper OHPs because water $\left(\mathrm{H}_{2} \mathrm{O}\right)$ is relatively opaque to neutrons as compared to the copper container of the OHP. For example, Zhang et al. [16] demonstrated that the 
thermal performance of a water-charged (50\% volume fraction) copper FP-OHP is enhanced by the addition of a hydrophilic coating of cupric oxide $(\mathrm{CuO})$ nanostructures on either the evaporator or condenser sections, as compared with an untreated FP-OHP. Moreover, they correlated the improved performance with the enhancement of thin-film evaporation (condensation) in the evaporator (condenser) coated with $\mathrm{CuO}$ nanostructures.

Wettability is believed to be a significant factor in OHP performance, as discussed in Secs. 1.2 and 1.3. For example, Hao et al. [23] used high-speed photography to measure the water slug movement within various copper FP-OHPs coated with $\mathrm{CuO}$ nanostructures whose wetting ranged from hydrophilic to hydrophobic. They determined that the hydrophilic surfaces promoted faster and larger amplitude slug oscillations and therefore better performance compared with untreated-copper and hydrophobic surfaces. Their results are consistent with the model developed by Ma et al. [173], which predicts the dependence of OHP thermal performance on the fluid oscillations. However, Hao et $a l$. required a polycarbonate window to conduct their imaging experiments, which is expected to exhibit different wetting properties compared to copper or $\mathrm{CuO}$ surfaces.

\subsubsection{Thermal Performance Measurements}

Two copper FP-OHPs have been fabricated and assembled at the University of Missouri. Each OHP consists of two flat, rectangular $\left(88 \times 50 \mathrm{~mm}^{2}\right)$ copper plates: a top cover plate ( $1 \mathrm{~mm}$ thick) and a bottom plate $(2.5 \mathrm{~mm}$ thick), the latter having a rectangular groove $(1 \mathrm{~mm}$ wide $\times 1.5 \mathrm{~mm}$ deep) machined into the surface that makes 12 parallel turns between the evaporator and condenser. For the CuO-coated FP-OHP, hydrophilic 
$\mathrm{CuO}$ nanostructures (see Fig. 2.5) were grown on the FP-OHP channel surface by immersing the $\mathrm{OHP}$ into a hot $\left(95^{\circ} \mathrm{C}\right)$ alkaline bath for 10 minutes [56]. The device was then sealed with the copper cover plate using an indium O-ring. We have previously characterized the microscopic interaction between this $\mathrm{CuO}$ coating and its hydration water using incoherent neutron scattering, neutron diffraction, and electron microscopy techniques $[\mathbf{1 6 8}, \mathbf{1 7 4}]$ as described in previous chapters. Our results show that the $\mathrm{CuO}$ coating provides a strongly hydrophilic interface which affects the wetting, freezing and melting behavior, dynamics, and structure of water near the nanostructures.

The thermal performance of an operating OHP can be characterized by the temperature difference between its evaporator and condenser sections. S. Stover has measured the thermal performance of both the untreated and CuO-coated copper FPOHPs [175]. In these tests, heat was input to the FP-OHP at the evaporator by cartridge heaters while the condenser was held to $20^{\circ} \mathrm{C}$ by a separate heat exchanger. Five thermocouples were placed along the outside of the FP-OHP to monitor the device's temperature: two each on the evaporator and condenser and one in the adiabatic section. Heat was applied incrementally by the cartridge heaters, from $0 \mathrm{~W}$ to $230 \mathrm{~W}$, and the temperatures of all thermocouples were recorded continuously. Deionized water was used as the working fluid at a $65 \%$ filling ratio ( $65 \%$ full by weight). The average temperature difference between the evaporator and condenser is plotted in Fig. 5.1 versus heat input (in watts) at the evaporator. These results indicate that, above a heat input of $30 \mathrm{~W}$ the CuO-coated FP-OHP outperforms the untreated FP-OHP as indicated by the reduction in the temperature difference $\Delta T$ between the evaporator and condenser. 


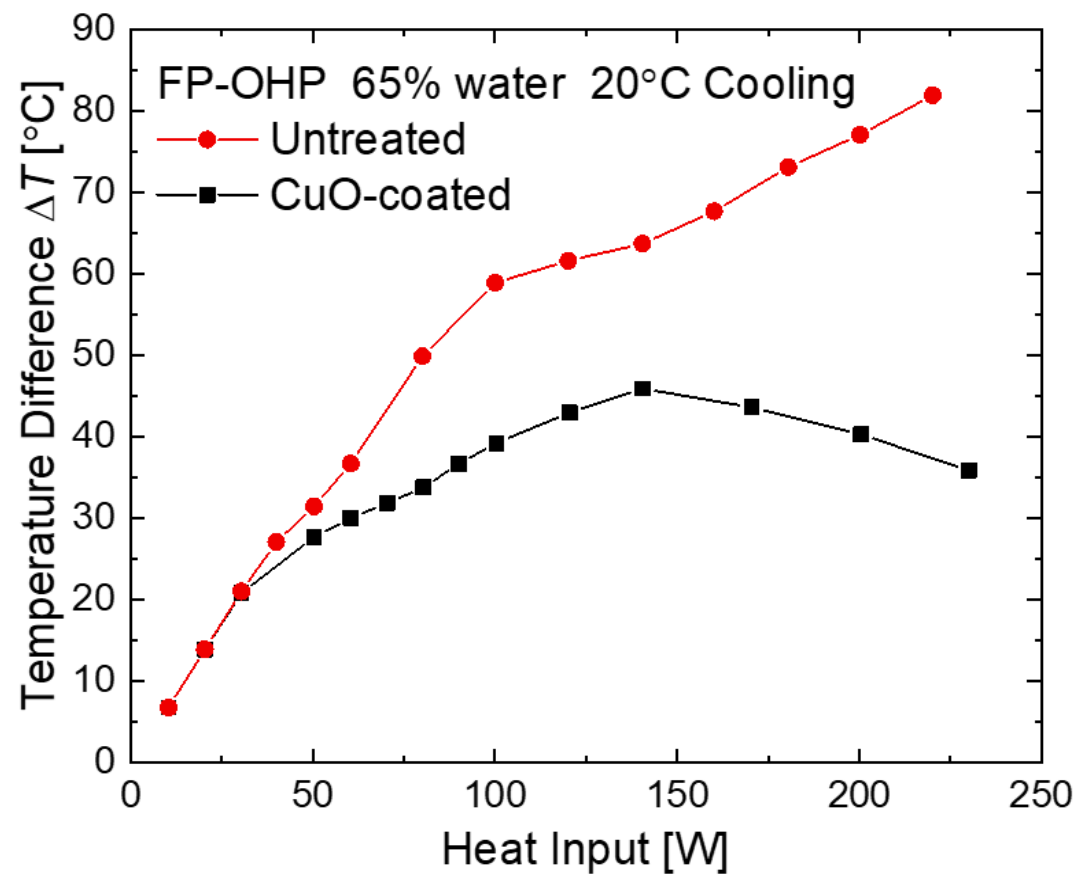

Figure 5.1. Average temperature difference $\Delta T$ between the evaporator and condenser sections of an FP-OHP (see Fig. 1.3a) as a function of heat input at the evaporator for untreated (red circles) and $\mathrm{CuO}$-coated (black squares) FP-OHPs (see Fig. 1.3b). The FP$\mathrm{OHP}$ was vertically oriented with the evaporator directly below the condenser. Also, the condenser was held to a fixed temperature of $20{ }^{\circ} \mathrm{C}$ and the OHP was filled to $65 \%$ water by weight. Adapted from [175], courtesy of S. Stover.

\subsubsection{Neutron Imaging Measurements}

At the Neutron Imaging Facility CG-1D at ORNL's HFIR [170], we measured the neutron beam attenuation by the FP-OHP as well as the image contrast produced by liquid $\mathrm{H}_{2} \mathrm{O}$ within its channels. In this test, the $\mathrm{OHP}$ and its supporting equipment were oriented perpendicular to the incident beam, and images were recorded by the CCD detector in a series of five-second exposures. A representative neutron radiograph is shown in Fig. 5.2. The liquid slugs in the channels near the bottom of the image can be distinguished from the copper OHP as shown by the line scan (grey-scale intensity vs. pixel position along the red dashed line) in Fig. 5.2. The amount of liquid water in the 
OHP is unexpectedly lower than the 50\% filling ratio intended for testing, which could have been caused by a leak during shipping. This issue will be addressed before additional measurements.

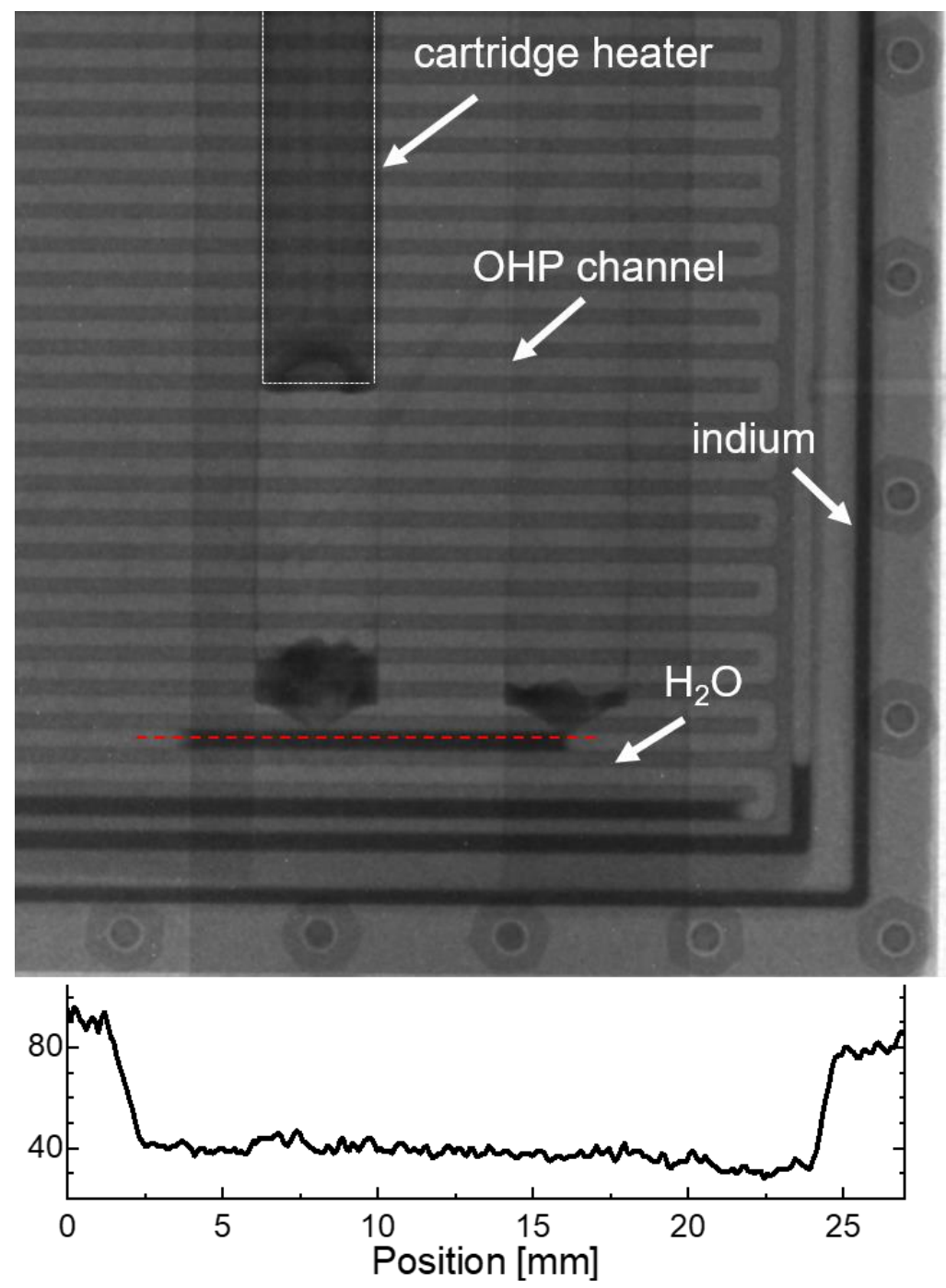

Figure 5.2. Neutron radiograph of the evaporator section of a copper FP-OHP partially filled with water. The image was corrected for inhomogeneities in the neutron beam and detector by normalization against open beam and background images. The line scan below the neutron radiograph shows the grey-scale image intensity vs. position through a liquid slug (red dashed line) [176] demonstrates the image contrast between liquid ( 40) and vapor/copper components ( 80). 
Based on preliminary thermal performance measurements [175] (Fig. 5.1), we hypothesize that the hydrophilic $\mathrm{CuO}$ coating from Ref. [56] facilitates larger amplitude and higher frequency oscillations of the water slugs as indicated by the drop in the temperature difference $\Delta T$ a heat input of $30 \mathrm{~W}$. To characterize the amplitude and frequency of the water slug oscillations, we propose to obtain neutron videos in operando at a rate of $30 \mathrm{~Hz}$ for water-charged $\left(65 \% \mathrm{H}_{2} \mathrm{O}\right.$ by weight $) \mathrm{FP}-\mathrm{OHPs}$ both with and without a nanostructured-CuO coating. The proposed frame rate of $30 \mathrm{~Hz}$ has been shown to resolve the rapid water slug oscillations $[\mathbf{1 6 , 1 7 2}]$. Neutron videos will be recorded as power (heat) is incrementally increased at the evaporator as in the preliminary work (Fig. 5.1) [175]. Temperature data from the thermocouples placed along the OHP's outer surface will be saved within the metadata of each image file to quantify the OHP thermal performance offline. Video analysis will proceed by first correcting for inhomogeneities in the neutron beam across the detector by normalizing individual images to open-beam and background intensities (see, e.g., Fig. 5.2) using standard reduction algorithms available at ORNL. In each video frame, a line scan of each channel of the OHP (pixel intensity vs. position) will allow discrimination of liquid slugs by their image contrast (see, e.g., line scan in Fig. 5.2). The evaporator and condenser will be identified in an image by the footprint of the heating and cooling blocks, respectively, and by the location of the thermocouples on the opposite face. In this way, the slug oscillations (amplitude and frequency) can be measured versus time and/or heat input. 


\subsection{Conceptual Design of a Thermal Neutron Imaging}

\section{Facility at the MU Research Reactor}

The University of Missouri Research Reactor (MURR) hosts the most powerful university-based research reactor in the U.S. and currently operates at $10 \mathrm{MW}$ for 6.5 days/week, 52 weeks/year. Researchers at MURR are seeking to construct a first-of-itskind Center for Dynamic Imaging (CDI) with the aim of advancing agriculture and plantbased sciences. At the heart of the CDI is a thermal neutron imaging facility at MURR that will enable state-of-the-art neutron imaging capabilities. Furthermore, the CDI will house and operate other in-situ imaging capabilities such as x-ray, gamma-ray, and planar PET (Positron Emission Tomography). Recently, MU was invited to submit a Mid-Scale Research Infrastructure-1 proposal to the NSF (National Science Foundation) in May 2019 to support these initiatives. In this section, we introduce the concepts needed to design a neutron imaging facility and follow with a summary of the critical optical components of the MURR Neutron Imaging Station (NIS).

Construction of the NIS is driven by the need for multimodal and non-destructive characterization of complex dynamic processes involved in the uptake of water and nutrients of plant life. These processes span time and length scales of $10^{1}-10^{6}$ seconds and $10^{-6}-10^{0}$ meters, respectively. Accordingly, the NIS should provide capabilities for high flux-on-sample, high-spatial-resolution, and large field-of-view imaging modes. Upon construction of the CDI, MU and MURR will be positioned to advance plant phenotyping and diagnostics into a quantitative science to address local, national, and global challenges in food security and nutrition. 


\subsubsection{MURR Design and Performance}

MURR is a medium-power, light-water cooled/moderated research reactor designed to support the research, education, and service mission of the University of Missouri. As a reliable resource, MURR supports many branches of research including neutron scattering $[\mathbf{1 3 0}, \mathbf{1 6 8}, \mathbf{1 7 4}, \mathbf{1 7 7}-\mathbf{1 7 8}]$, neutron activation analysis [179], radioisotope production [180-181], radiation therapy [182], archaeometry [183], and nuclear forensics [184] to cite a few applications. As illustrated in Fig. 5.3, the core consists of eight, 75 $\mathrm{cm}$-tall, highly enriched ${ }^{235} \mathrm{U}$ fuel elements organized into a $30-\mathrm{cm}$ diameter cylinder, which is submerged 10-m deep in a pool of deionized water [185-187]. Fission reactions within the core produce fission fragments, gamma rays, charged particles, and highenergy $(\geq \mathrm{MeV})$ free neutrons [185]. A fraction of the neutrons lose energy by scattering from hydrogen towards thermal equilibrium with the moderator, i.e., "thermalize" ( $E \sim k_{B} T$ where $k_{B}$ is the Boltzmann constant and $T$ is temperature). Given the average moderator temperature of $136^{\circ} \mathrm{F}(\sim 331 \mathrm{~K})$ at MURR, the peak brightness of neutrons exiting the surface of the moderator occurs at $\sim 28.5 \mathrm{meV}$. While the energy spectrum of a thermal reactor is continuous, it is nonetheless convenient to categorize neutrons based on their mean energies, as given in Table 5.1.

\begin{tabular}{c|c|c|c}
\multicolumn{1}{c}{} & Neutron Energy & Wavelength $[\AA]$ & Velocity [m/s] \\
\cline { 2 - 4 } Cold/Slow & $<12 \mathrm{meV}$ & $>2.61$ & $<1513$ \\
Thermal & $12-100 \mathrm{meV}$ & $0.90-2.61$ & $1513-4370$ \\
Epithermal & $100 \mathrm{meV}-1 \mathrm{eV}$ & $0.29-0.90$ & $4370-13819$ \\
Hot/Fast & $>1 \mathrm{eV}$ & $<0.29$ & $>13819$
\end{tabular}

Table 5.1. Exemplary neutron energy ranges with corresponding wavelength $(\lambda \sim$ $9.045 / \sqrt{E(\mathrm{meV})})$ and velocity $(v \sim 437 \sqrt{E(\mathrm{meV})})$ conversions. 

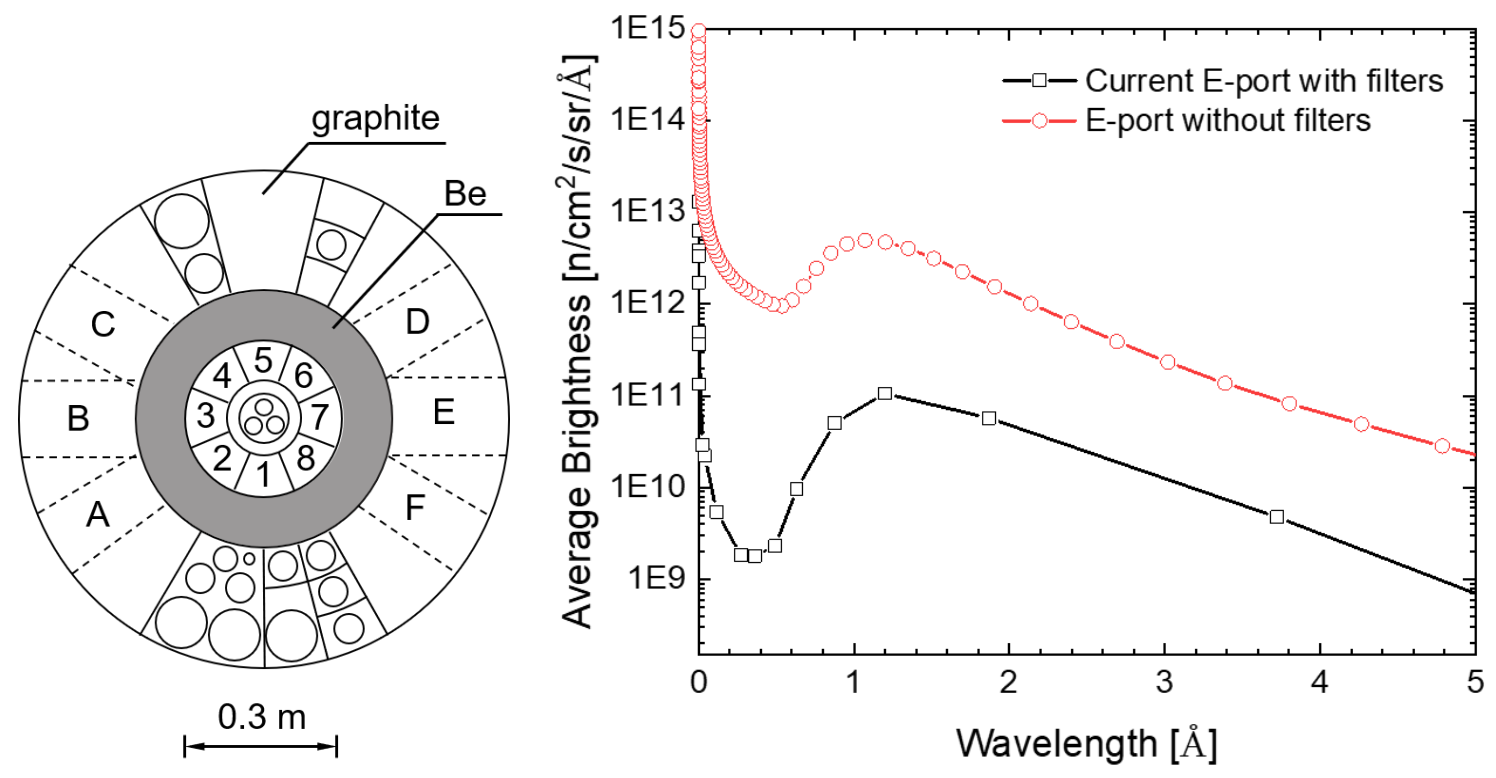

Figure 5.3. (left) Plan view of the MURR core (as of 1983) containing eight 235U fuel elements in a cylindrical arrangement, surrounded by neutron-reflector material consisting of beryllium $(\mathrm{Be})$ and graphite wedges. Six ports $(\mathrm{A}-\mathrm{F})$ are used to extract neutrons from the core. Adapted from Refs. [186-187]. (right) Neutron brightness vs. wavelength at E-port before [188] and after removing filters currently in place. See Appendix D for details.

At MURR, neutrons are extracted radially from the core in a series of six beam tubes, or ports $(\mathrm{A}-\mathrm{F})$, that extend from the Be reflector, through graphite wedges, to the exterior of the concrete frame (biological shielding). The power spectrum for E-port is shown in Fig. 5.3 in the current configuration with filters [188] and without filters. The average brightness without filters was calculated by scaling the current brightness of Eport to that of the HB-2 beamline at the HFIR at ORNL [189], which has a comparable geometry. Fig. 5.4 shows a schematic of the beam-port floor (BPF) at MURR and the arrangement of end-stations. For the conceptual design of the NIS at E-port, the primary size constraints are 1) the maximum length set by the 20-m distance from E-port to the North wall and 2) the height of the vestibule $(3.96 \mathrm{~m})$. Weight restrictions are based primarily on the load capacity of the overhead crane (15 metric tons). 


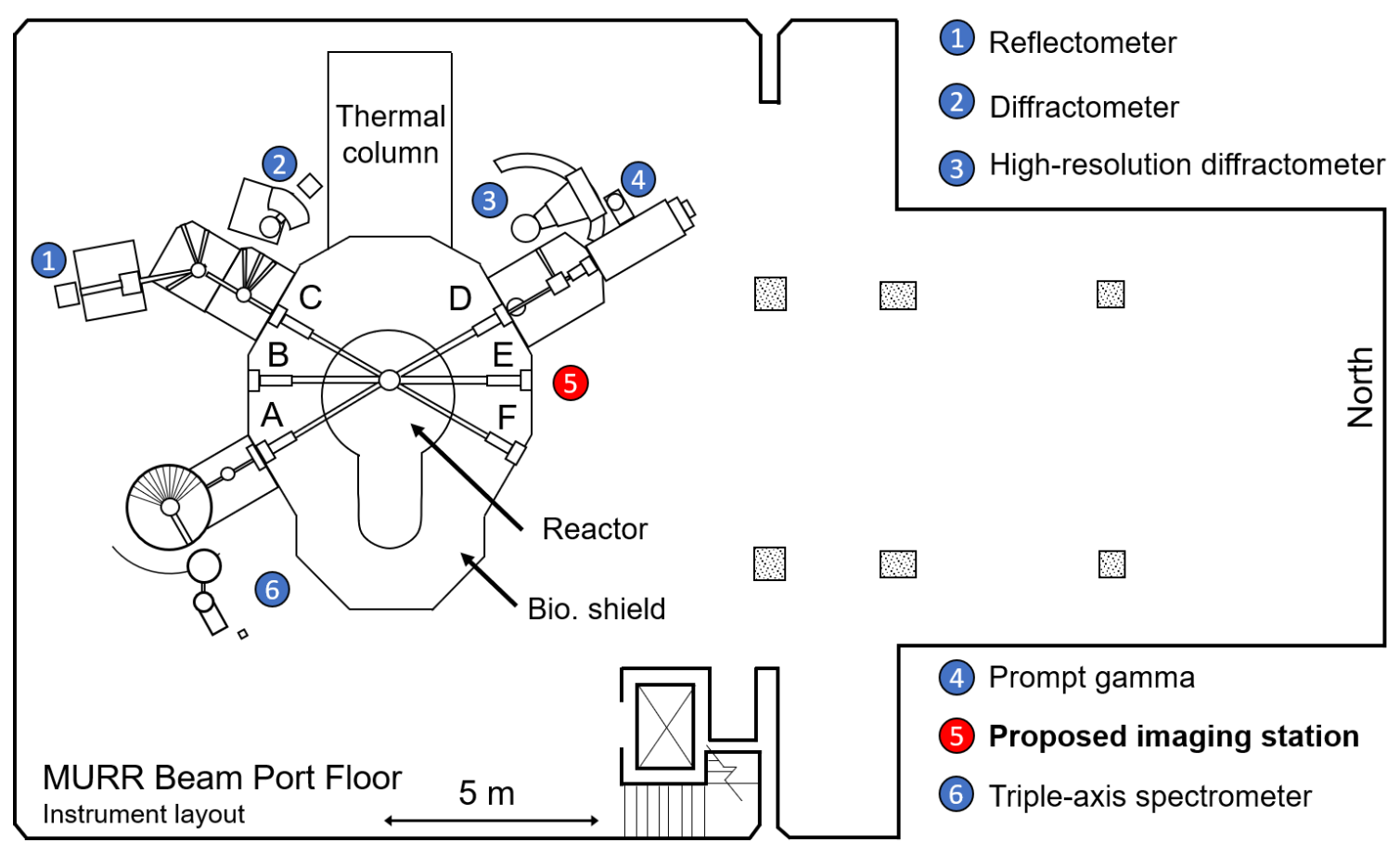

Figure 5.4. Plan view of the MURR beam-port floor (BPF) shown with the current suite of instruments. Courtesy of D. Nickolaus.

\subsubsection{Preliminary Calculations}

Optical components of the NIS are optimized to provide the maximum available neutron flux on a large field-of-view (FOV), suitable for imaging part of fully-grown plants. This is achieved by using the entire effective size of the neutron source $(\sim 15.2 \mathrm{~cm}$ diameter) shaped by the front-end of the cylindrical beam-tube. Like most neutron imaging facilities, the NIS is designed with a pinhole-camera-type geometry (Fig. 5.5), where the spatial resolution is largely defined by the diameter $(D)$ of the aperture placed at a distance $L$ from the detector; This is measured as the quantity $L / D$ where a higher $L / D$ means lower beam angular divergence and thus higher spatial resolution. In this regard, $L / D$ should be maximized, but neutron intensity decreases proportionally to $D^{2} / L^{2}$, so one must compromise between high-flux and high-spatial-resolution. 

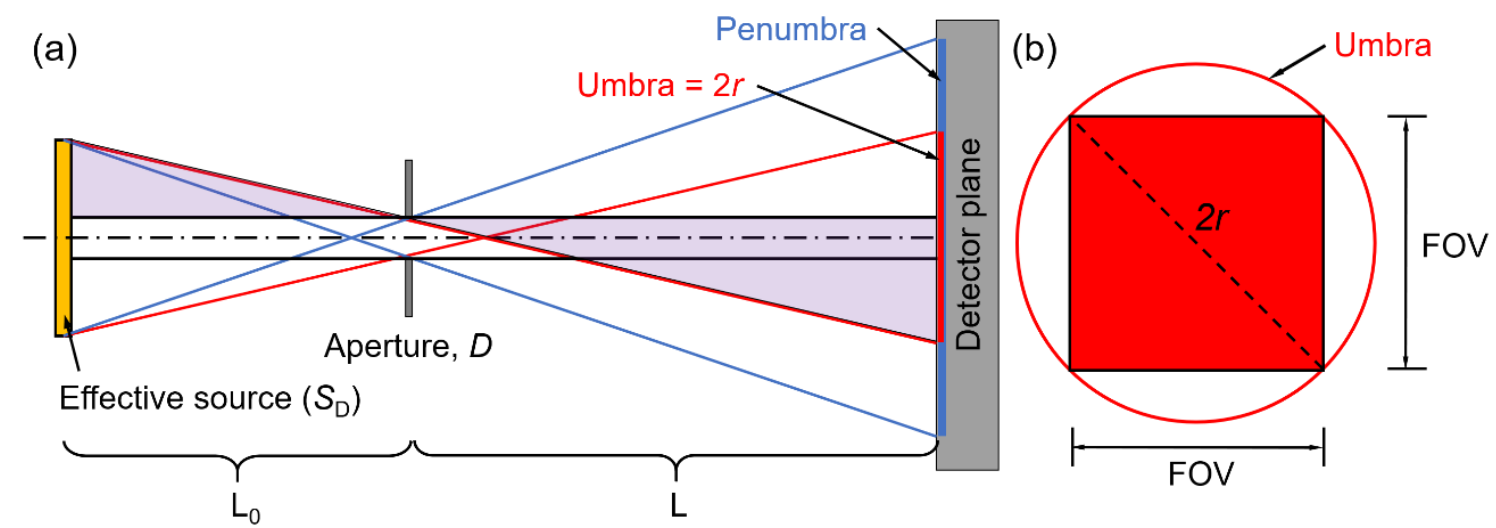

Figure 5.5. (a) Schematic of a pinhole-camera geometry used to determine the optimal diameter $(D)$ and location $(L)$ of the aperture. A set of similar triangles is outlined in purple. The "effective source" models the diameter $\left(S_{D}\right)$ of the beam tube starting from the face of the Be reflector (see Fig. 5.3). (b) Geometric relationship between the region of full illumination (umbra) and a square field-of-view (FOV) typically used by neutron area detectors.

Pinhole-camera geometry is illustrated in Fig. 5.5a and includes two sets of rays that define the outer boundaries of the penumbra (blue) and umbra (red). Every point within the umbra receives equal illumination by the source - a requirement for optimal neutron imaging. Fig. 5.5b shows the geometric relationship between the conical umbra and a square-FOV. Using the maximum source diameter $\left(S_{D}\right)$ of $15.2 \mathrm{~cm}$ and placing the aperture as close to the biological shielding as possible $\left(L_{0}=334 \mathrm{~cm}\right)$, we can set up an equation to solve for the diameter of the umbra,

$$
2 r=\left[\frac{L}{L_{0}}\left(S_{D}-D\right)-D\right]
$$

Note the conversion from umbra-diameter $(2 r)$ to a square-FOV: $2 r=\sqrt{2} F O V$ (see Fig. 5.5b). There is also a mutual relationship between $L$ and $D$, namely $L / D=c$ (constant); thus, by using $L$ as a free parameter, we can solve for the umbra's diameter as shown graphically in Fig. 5.6. Generally, the size of the umbra increases with increasing $L$ out to 15 meters. Given the limited space around E-port, $L$ will be a maximum of 10 meters. 


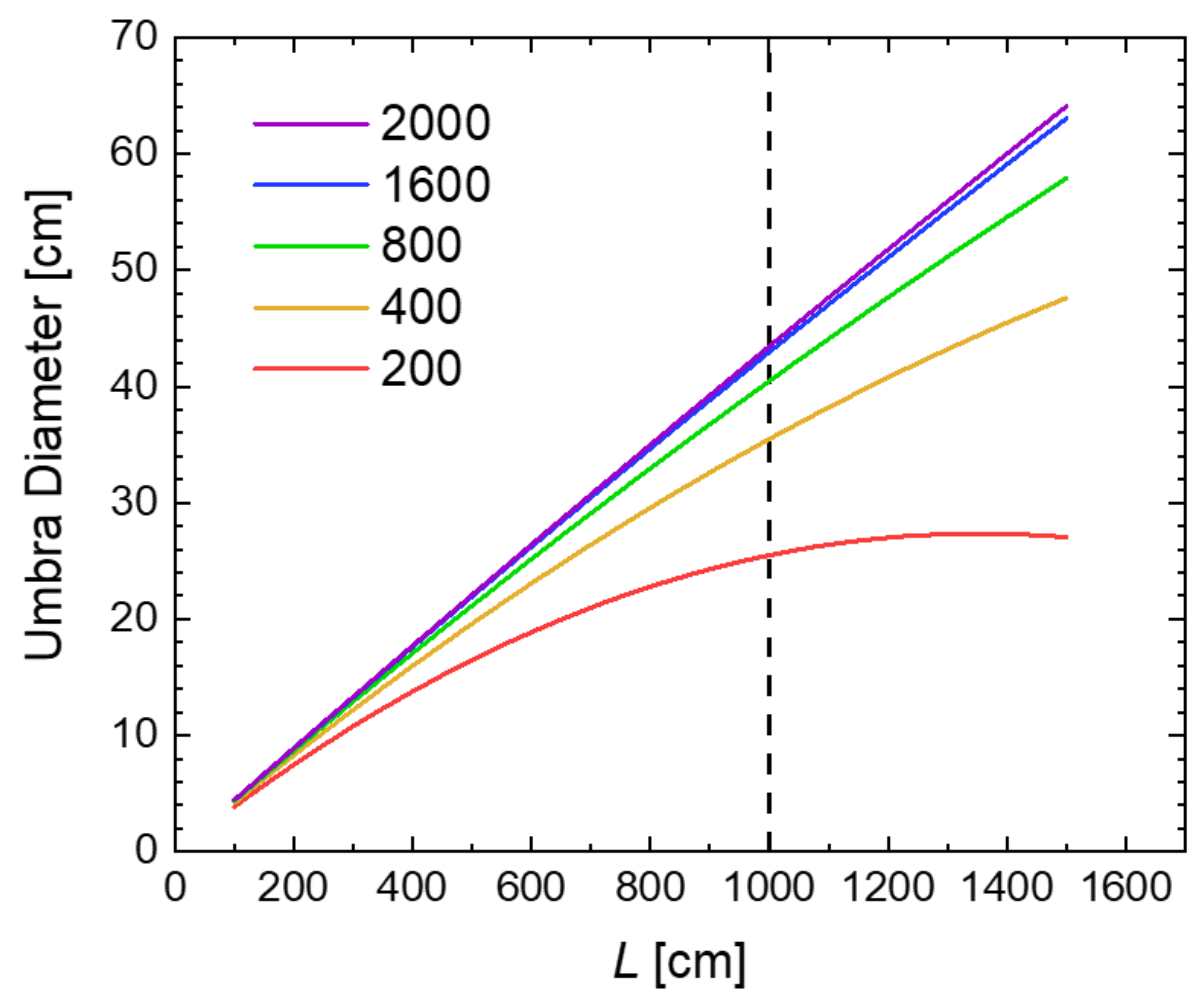

Figure 5.6. Diameter of the umbra of the neutron beam at the detector position vs. aperture-detector distance $L$ for selected $L / D$ settings.

A method to enlarge the FOV at small $L / D$ (high-flux, low-resolution) is to place a second aperture with a fixed diameter upstream from the first aperture closer to the front of the beam tube. This concept was adapted from that of the future time-of-flight neutron imaging station VENUS at ORNL's Spallation Neutron Source [190]. A reasonable location for this second fixed aperture is inside of the biological shielding, 215 $\mathrm{cm}$ from the front of the beam tube. Positioning this aperture inside of the biological shielding also serves to reduce the number of unwanted neutrons that would otherwise exit the beam port and require additional beamline shielding. The shape of the aperture (tapered hole) is designed so that it does not obstruct neutrons that reach the detector for the other $L / D$ settings selected by the downstream aperture system. Following the same procedure as for the first aperture, the diameter of the umbra at the detector plane is $\sim 9.5$ 
$\mathrm{cm}$ for a fixed $L / D$ of 100 . A summary of the proposed aperture sizes and the corresponding instrument performance is given in Table 5.2. The length of the proposed instrument, from the biological shielding to the end of the beam-stop, is $\sim 12.1 \mathrm{~m}$. The distance from the Be-reflector to the image/detector plane is $1334 \mathrm{~cm}$.

\begin{tabular}{ccccc} 
Aperture & $\boldsymbol{L} / \boldsymbol{D}$ & $\boldsymbol{D}[\mathrm{cm}]$ & Umbra diameter $[\mathrm{cm}]$ & FOV $[\mathrm{cm}]$ \\
\hline Fixed & 100 & 11.23 & 9.51 & 6.72 \\
\hline & 200 & 5 & 25.50 & 18.03 \\
& 400 & 2.5 & 35.47 & 25.08 \\
Variable & 800 & 1.25 & 40.46 & 28.61 \\
& 1600 & 0.625 & 42.95 & 30.37 \\
& 2000 & 0.5 & 43.45 & 30.72
\end{tabular}

Table 5.2. Instrument settings and performance. See Fig. 5.5b for the conversion between umbra diameter and FOV.

\subsubsection{Instrument Layout}

In the previous section, we discussed reasonable sizes and locations of both apertures and the maximum length of the NIS at E-port. In addition, the experimental area at the exit of the flight tube should include a large $\left(2 \times 2 \mathrm{~m}^{2}\right)$ floor space to accommodate fully grown, agriculturally relevant plants such as dent corn as dent corn (Zea mays var. indentata), sorghum, soybeans, and more. Furthermore, the space must allow for a platform of multiple detectors around the sample position for multimodal neutron, $\mathrm{x}$-ray, gamma-ray, and planar-PET (Positron Emission Tomography) imaging.

Since E-port directly faces the fuel elements of the core, multiple radiation filters and shields are added to reduce contamination from neutron and gamma radiation. Note that the selection of these materials for this conceptual design of the NIS precede detailed radiation transport calculations (e.g., Monte Carlo N-Particle (MCNP)) used to furnish 
shielding type, thickness, and placement in the facility. We therefore based the following designs on those of other major neutron imaging facilities [190-193]. More detailed descriptions of commonly used filters and shields are provided in Sec. 5.2.4.

The NIS component layout is shown schematically in Fig. 5.7 and Fig. 5.8. In the current configuration of E-port, a permanent cylindrical beam tube is mounted inside of the biological shielding where the first $\sim 200 \mathrm{~cm}$ are practically inaccessible. The frontend of the tube butts against the beryllium (Be) reflector and has a diameter of about 6 inches $(15.24 \mathrm{~cm})$, which is the effective size of the neutron source.

As discussed in the previous section, an aperture with a fixed diameter $(11.23 \mathrm{~cm})$ is placed as close as possible $(215 \mathrm{~cm})$ to the source in order to maximize neutron flux for experiments that require a high signal-to-noise ratio in a short period of time at the detector at the lowest spatial resolution setting. The aperture's secondary role is to collimate and attenuate undesired radiation inside of the biological shielding. The tapered bore of the aperture is designed to pass only the neutron rays that produce homogeneous illumination of the detector.

To decrease the number of epithermal and fast neutrons in the beam, a 5-inch diameter/thick, single crystal of sapphire $\left(\mathrm{Al}_{2} \mathrm{O}_{3}\right)$ will be mounted inside of a stainlesssteel tube, for example, and placed downstream coaxially to the fixed-aperture $262 \mathrm{~cm}$ from the front of the beam tube. As discussed further in Sec. 5.2.6, there is no significant increase in neutron flux if the sapphire were cooled to cryogenic temperatures. Therefore, it can be placed up against the fixed aperture inside of the biological shielding. During the engineering design phase of the NIS construction, additional calculations will be performed to estimate the temperature increase of the crystal while it is in the beam. 
After allowing for a $\sim 30$-cm thick primary shutter system, the resolution-defining aperture is placed as close to the biological shielding as possible, which is $334 \mathrm{~cm}$ from the front end of the beam tube. Given the wide range of time and length scales of experiments planned for the NIS, it is prudent to install an aperture-selecting system in which the aperture size $D$ can be quickly and easily changed based on the nature of the experiment (flux vs. resolution). The conceptual design of this system is simply a $\sim 1-\mathrm{cm}$ thick plate of neutron absorbing material (e.g., boron carbide or boral) with circular holes drilled-out. For each $L / D$ setting, the corresponding hole of diameter $D$ will be translated, or rotated, into the beam.

In addition to epithermal and fast neutrons, gamma radiation contaminates the neutron beam and can degrade the performance of detectors and other electronics downstream. Furthermore, gamma-rays with energies in the $\mathrm{MeV}$ range can be mistaken for neutron-capture events in some types of neutron detectors, leading to changes in image contrast or "fogging" [194]. Unlike neutrons, gamma radiation interacts with matter via electromagnetic forces and can therefore be attenuated by using high-Z materials such as lead or bismuth. In this case, we propose a 5-inch diameter/thick, single crystal of bismuth (Bi) (atomic number 83) whose front face is located $335 \mathrm{~cm}$ from the source. As will be discussed in Sec. 5.2.6, it is advantageous to cool bismuth to liquidnitrogen temperatures $(\sim 80 \mathrm{~K})$. Therefore, the crystal will be housed in a cryostat connected to a liquid-nitrogen source in a manner similar to that used at the NIST facility [191].

Immediately downstream from the Bi filter is another beam scraper designed to stop radiation that is not used for image formation. The form factor of the collimator is a 
60-cm long cylinder where the circular face is normal to the neutron beam and placed $\sim 366 \mathrm{~cm}$ from the source. Tapered holes will be drilled through the collimator to pass neutrons that make up the umbra at the detector plane. The diameters of these holes will depend on the sizes of the apertures; upon selection of an aperture, the collimator barrel will rotate about the long axis to place the appropriately sized hole into the beam.

A long, cylindrical tube (flight tube) is added between the rotating collimator and the experimental area in order to minimize neutron intensity loss from air scattering. Accordingly, the tube will either be evacuated via mechanical pump or filled with a low neutron-attenuating gas like helium. In the current version of the NIS, there are three segments of the flight tube with the following dimensions: in order, 1) 3-ft long and 6inch inner-diameter (ID), 2) 9-ft long and 12-inch ID, and 3) 9-ft long and 18-inch ID. To attenuate the rays of the penumbra that fall outside of the detector's sensitive area and the diameter of the beam stop, the inner walls of the tubes can be lined with a neutron absorbing material. Furthermore, slits (e.g., beam "scrapers" or "limiters") can be added to help shape the beam that exits the flight tube. The end of the NIS is marked by a beamstop whose composition is to be determined. 


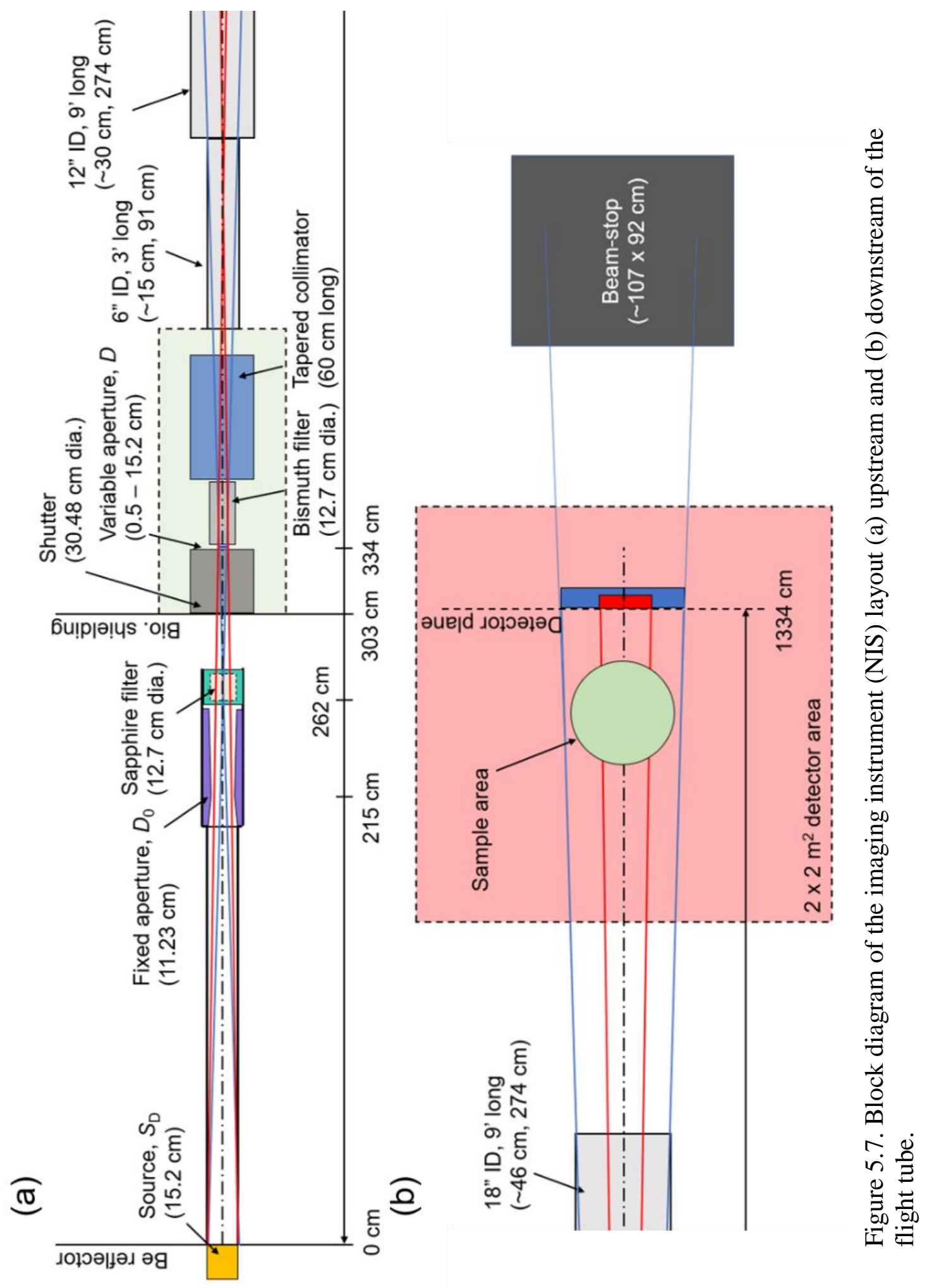




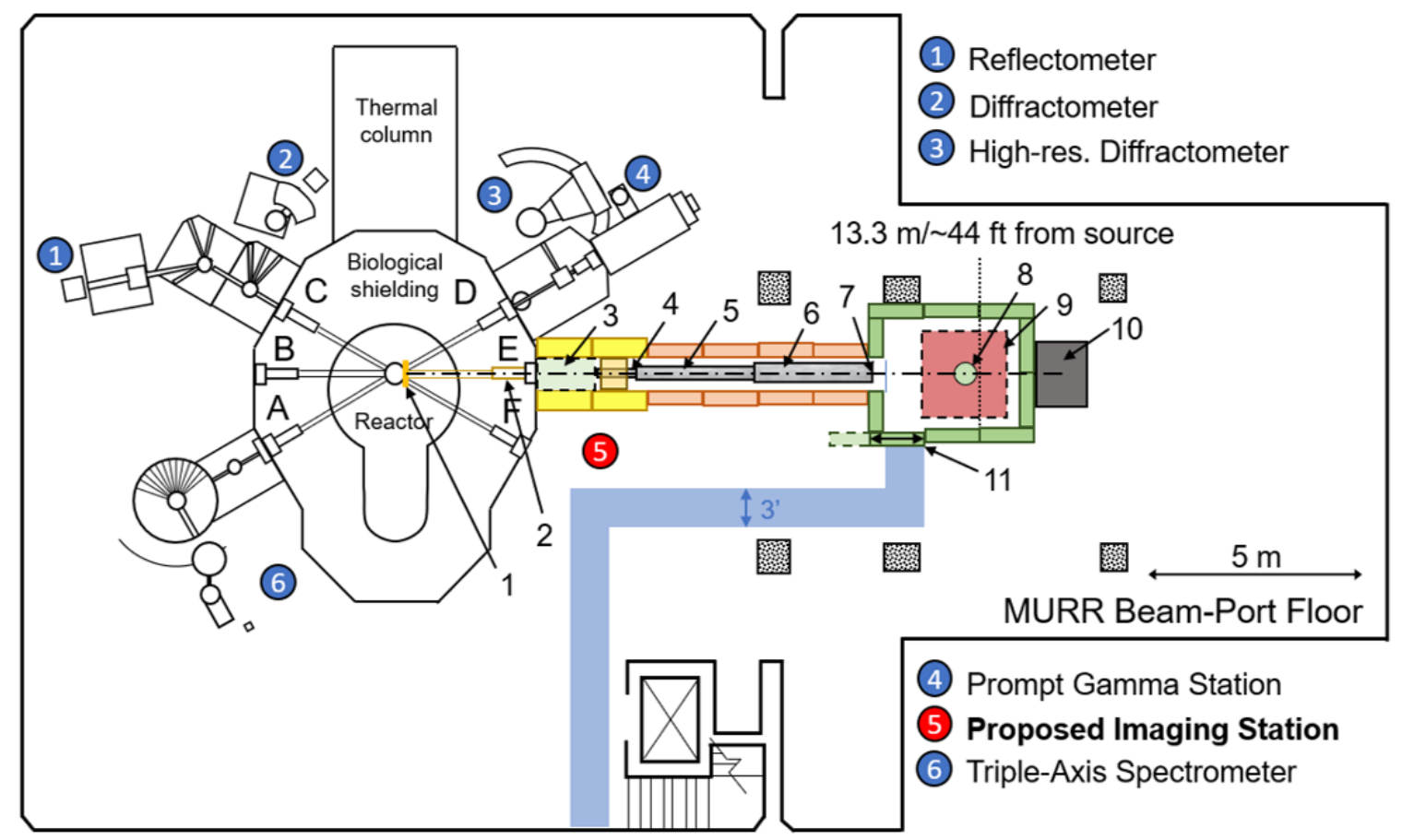

1. Effective source (6" dia.)

6. Flight tube 3 (18" ID, 9' long)

2. Inner collimator (fixed aperture/ sapphire filter)

7. Adjustable slit package

8. Sample position

3. Primary and secondary shutters/ Bi-filter/variable aperture (4.5' $\times 2.5^{\prime}$ area)

9. Sample/detector area ( 6.5' x 6.5')

4. Flight tube 1 (6" ID, 3' long)

5. Flight tube 2 (12" ID, 9' long)

10. Beam-stop (4' $\left.\times 5^{\prime}\right)$

11. Sliding access door

Figure 5.8. Detailed layout of the existing instrument suite at MURR including the proposed NIS at E-port. Critical components have been identified and listed numerically. 


\subsubsection{Neutron and Gamma-Ray Filters and Shielding}

Radiation in the experimental area of the proposed NIS consists of a spectrum of neutrons and gamma-rays from the core as well as secondary photons and other particles emitted from neutron-capture events in materials placed in the beam. Experiments outside of the NIS can act as sources of background radiation. In this section, we discuss the materials used as filters to attenuate neutrons and gamma rays while letting thermal neutrons pass relatively freely. Also, this section includes common materials that are used as radiation shielding to prevent radiation from escaping to, and intruding from, outside of the NIS.

\section{Filters}

The transmitted neutron flux $T$ through any material is approximated by a simple expression [194] (Lambert-Beer law),

$$
\frac{T}{T_{0}}=\exp (-N \sigma d)
$$

where $T_{0}$ is the incident neutron flux $\left(\mathrm{cm}^{-2} \mathrm{~s}^{-1}\right) ; N$ is the number density of the material (e.g., atoms per $\left.\mathrm{cm}^{-3}\right)$; $\sigma$ is the total microscopic cross-section measured in units of barns $\left(10^{-24} \mathrm{~cm}^{2}\right)$; and, $d$ is the thickness or penetration depth $(\mathrm{cm})$. The guiding principle in choosing radiation filters is to control these parameters to pass only the desired radiation (e.g., thermal neutrons).

The cross section $\sigma$ does not represent the real size of a nucleus; rather, it is an effective size based on the probability of interaction with a neutron. The total 
microscopic cross-section of a crystalline solid varies as a function of neutron wavelength ( $\lambda$ ) and is given as a sum [195],

$$
\sigma(\lambda)_{t o t a l}=\sigma(\lambda)_{a b s}+\sigma(\lambda)_{i n e l}+\sigma(\lambda)_{e l}
$$

where $\sigma_{a b s}$ is the absorption cross-section corresponding to neutron-capture events by the target nuclei; $\sigma_{\text {inel }}$ is the inelastic scattering cross-section related to neutron-target interactions in which the total kinetic energy changes. This interaction includes two parts: single- and multi-phonon scattering. The latter can be neglected for neutron energies $E \ll$ $k_{B} \theta_{D}$, where $k_{B}$ is the Boltzmann constant and $\theta_{D}$ is the Debye temperature of the target material. (The Debye temperatures of several common filter materials from Ref. [194] are listed in Table 5.3). The last component, $\sigma_{e l}$, is the scattering cross-section related to elastic scattering in which the total kinetic energy of the neutron-target interaction is unchanged. The total scattering cross-sections of a few selected isotopes are shown in

\section{Fig. 5.9.}

\begin{tabular}{cc} 
Material & Debye temperature $\theta_{D}[\mathrm{~K}]$ \\
\hline Beryllium $(\mathrm{Be})$ & 1100 \\
Graphite $(\mathrm{C})$ & 1050 \\
Sapphire $\left(\mathrm{Al}_{2} \mathrm{O}_{3}\right)$ & 1040 \\
Silicon $(\mathrm{Si})$ & 420 \\
Bismuth $(\mathrm{Bi})$ & 300 \\
Lead $(\mathrm{Pb})$ & 280
\end{tabular}

Table 5.3. Empirical Debye temperatures of several crystalline materials. Adapted from Ref. [195]. 


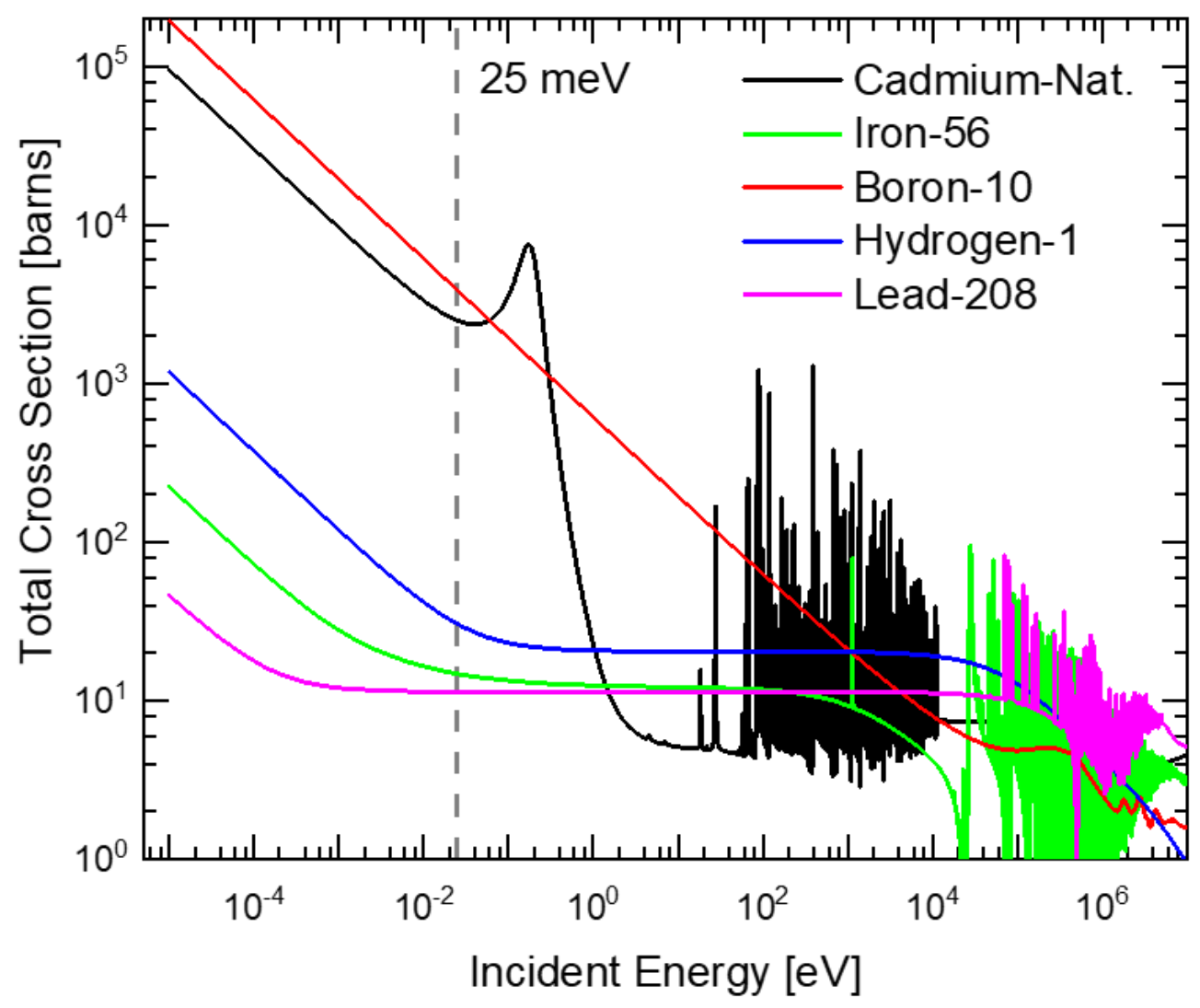

Figure 5.9. Total microscopic cross-sections of naturally occurring cadmium, ${ }^{56} \mathrm{Fe},{ }^{10} \mathrm{~B}$, ${ }^{1} \mathrm{H}$, and ${ }^{208} \mathrm{~Pb}$. Data were reproduced from the ENDF/B-VIII.0 database from Ref. [196]. A dashed vertical line is drawn at $25 \mathrm{meV}$, which corresponds to the typical kinetic energy of thermal ("room-temperature") neutrons.

\section{Shielding}

The primary function of radiation shielding is to provide safe access for users around the NIS during experiments. In addition, shields need to minimize secondary radiation produced by components placed in the beam as well as background from experiments near the NIS, all of which affect dose levels and image quality at the detector. The choice of material(s) can vary between different facilities or institutions. One must consider a material's attenuation, activation, and mechanical properties as well as cost, weight, and availability. Listed below are some information about several shielding materials commonly employed at neutron and accelerator research facilities. This list is, of course, 
not exhaustive as the intention is to apply the following schemes and strategies to the design of the NIS.

\section{Boron and Cadmium}

Boron (specifically ${ }^{10} \mathrm{~B}$ ) is a common material used to absorb thermal neutrons due to its exceptionally large absorption cross-section $\left(\sigma_{a b s}\left({ }^{10} \mathrm{~B}\right) \sim 3835\right.$ barns for $2200 \mathrm{~m} / \mathrm{s}$ neutrons [196]) and low dose of secondary radiation. Reaction products from a neutroncapture event consist of an alpha particle and a lithium-7 ion, the latter of which promptly decays and produces a relatively low-energy $(0.48 \mathrm{MeV})$ gamma [197-198]. Pure boron is extremely difficult to produce in appreciable quantities due to its high reactivity and hardness [198]. Nevertheless, compounds like boron carbide $\left(\mathrm{B}_{4} \mathrm{C}\right)$, Boral $\left(\mathrm{B}_{4} \mathrm{C}\right.$ clad in aluminum), and boron nitride (BN) are used across the nuclear, military, and aerospace industries. Notably, boron carbide, also known as "black diamond," is an advanced ceramic material characterized by high melting point $\left(2427^{\circ} \mathrm{C}\right)$, corrosion resistance, and hardness $\left(3770 \mathrm{~kg} / \mathrm{mm}^{2}\right.$ Vickers hardness) [199]. Given its high hardness, $\mathrm{B}_{4} \mathrm{C}$ (and therefore Boral) is difficult to cut and form into custom sizes/shapes and therefore tends to be expensive compared to some of the other options.

Like boron-10, cadmium $(\mathrm{Cd})$ has a very large neutron absorption cross-section ( $\sigma_{a b s}(\mathrm{Cd}$-nat.) $\sim 2520$ barns for $2200 \mathrm{~m} / \mathrm{s}$ neutrons [95]), but the reaction byproducts include a shower of MeV gammas. Cadmium is a soft, malleable metal (density 8.65 $\mathrm{g} / \mathrm{cm}^{3}$ ) commonly used in the nuclear industry in shielding and control rods. Accordingly, it is available commercially in the form of wires, rods, ingots, and sheets (see, e.g., Ref. [200]). While cadmium demonstrates exceptional shielding performance in the thermal 
neutron energy range, many institutions are restricting its use due to its health hazards (toxic and known carcinogen).

\section{Hydrogen}

The mass of a neutron $(1.008664 \mathrm{u})$ is very close to that of a proton $(1.007276 \mathrm{u})$. Thus, a significant fraction of the kinetic energy of an incident neutron can be transferred to the hydrogen nucleus, which is essentially a proton. The average energy loss $E^{\prime}$ by a neutron, with an incident energy $E$, during an elastic collision with a nucleus of atomic weight $A$ is $E^{\prime}=2 E A /(A+1)^{2}[\mathbf{2 0 1}]$. Using hydrogen $(A=1)$, a $1 \mathrm{MeV}$ neutron loses about half of its energy after a single collision. After about 23 collisions, the neutron lies within the thermal range of neutron energies (see Table 5.1). Therefore, reactor moderators often consist of water, hydrogen, or deuterium $\left({ }^{2} \mathrm{H}\right)$.

Once high-energy neutrons have been thermalized by hydrogen, they can be scattered or absorbed by other compounds like those containing, for example, boron. In fact, a common strategy is to add boron to a compound with a high hydrogen content such as polyethylene $\left(\mathrm{CH}_{2}\right)_{n}$ (density $\left.\sim 0.92 \mathrm{~g} / \mathrm{cm}^{3}\right)$ [202]. Moreover, borated polyethylene is lightweight and can be machined with standard tools. Some other commonly used, Hheavy compounds include wood, earth, and paraffin wax $\left(\mathrm{C}_{\mathrm{n}} \mathrm{H}_{2 \mathrm{n}+2}\right)$ where each present both advantages and disadvantages in terms of applicability and safety (see, e.g., Ref. [202]). For example, the ${ }^{1} \mathrm{H}(\mathrm{n}, \gamma)^{2} \mathrm{H}$ reaction produces highly penetrative $(2.2 \mathrm{MeV})$ gammas [201-202], and wood and paraffin wax are both flammable if not treated properly. 


\section{Iron and Lead}

Despite the effectiveness of iron as a neutron attenuator/filter (see, e.g., Fig. 5.9), it should not be used in pure form as exposure to humid air leads to corrosion (rust) and therefore a high probability of lift-off (dust) contamination. Moreover, iron produces a number of troublesome secondary gammas, many of which in the 7.6 $\mathrm{MeV}$ range [203]. To remedy the corrosion problem, shielding is normally painted. Now, a commonly used method of protecting steel from corrosion is by using Macropoxy® (polyamide epoxy blend), which is typically used for marine applications. Another possibility is to use stainless steel (SS) $\left(\sim 7.8 \mathrm{~g} / \mathrm{cm}^{3}\right)$, which is safe, cheap, and widely available. Noteworthy is the nonmagnetic property of SS, which lends itself to experiments that are sensitive to magnetic fields. One must, however, take into account the long-lived ${ }^{60} \mathrm{Co}$ source of radioactivity from neutron irradiated SS.

Lead is perhaps the most common shield used to attenuate gamma radiation due to its high atomic number $(82)$ and density $\left(11.3 \mathrm{~g} / \mathrm{cm}^{3}\right)$ [202]. Many reactor and accelerator facilities, including MURR, have a stock of lead bricks and blankets on hand to reinforce existing safeguards/shields or to use during scheduled outage and maintenance activities. The main drawback in using lead is of course its toxicity to humans, introduced via inhalation and ingestion, which can be mitigated by painting. Bismuth is considered a non-toxic substitute for lead in some gamma-shielding applications. 


\section{Concrete}

Concrete is by-far the most common type of shielding material at reactor and accelerator facilities because it is cheap, widely available, non-toxic, and has favorable nuclear properties. Furthermore, it can be poured in place or formed into shapes or blocks. A couple short but good reviews on the nuclear properties of several types of concrete are given in Refs. [192,202]. While the neutron attenuation factor of concrete is low compared to boron or water, it can be improved by adding small amounts of heavy minerals such as magnetite $\left(\mathrm{Fe}_{3} \mathrm{O}_{4}\right)$, hematite $\left(\mathrm{Fe}_{2} \mathrm{O}_{3}\right)$, limonite $(\mathrm{FeOOH})$, serpentine $\left(3 \mathrm{MgO} \cdot 2 \mathrm{SiO}_{2} \cdot 2 \mathrm{H}_{2} \mathrm{O}\right)$, barite $\left(\mathrm{BaSO}_{4}\right)$, or colemanite $\left(\mathrm{Ca}_{2} \mathrm{~B}_{6} \mathrm{O}_{11} \cdot 5 \mathrm{H}_{2} \mathrm{O}\right)$ to the matrix [192]. Some important non-mineral additives include steel, lead, and boron. Note that with any chemical modifications, the mechanical properties of the product need to be evaluated.

In our conceptual design of the NIS, the wall and roof shielding consist of a mixture of steel-shot and paraffin wax, which is inserted into rectangular blocks whose dimensions are similar to those used at the NIST Neutron Imaging Facility (NIF) [191]. The special feature of these shielding blocks is that they can be bolted together into an interlocking solid structure in order to prevent direct line-of-sight or gaps through which neutrons or other radiation might escape to the BPF. For clarity, the shielding pieces are about $30 \%$ thicker than those used at the NIF, which probably overestimates the required thickness needed to reach minimum acceptable radiation levels. However, detailed radiation transport calculations will be required during the preliminary engineering design phase of the NIS construction to optimize the shielding configuration. 
The area around the exit of the beam port is expected to exhibit relatively higher levels of radiation (neutrons and gamma-rays) largely due to scattering and secondary emissions from filter, collimation, and shielding materials. Therefore, thicker blocks of shielding will be placed in this area. To isolate this relatively high radiation area from the rest of the beam line, a very thick (2-ft) block will be placed in-line some 5-ft downstream of the port. This shield is especially important for the low- $L / D$ settings where the penumbra is large. A circular hole will be cut into this block to accommodate the diameter of the flight-tube. Further downstream, the walls and rooves are presumably thinner, in this case $1-\mathrm{ft}$, and all sections are topped with 1 -ft-thick rooves. From the port to the end of the flight-tube, the walls will be 6-ft tall beyond which the height increases to 8 -ft around the sample/detector area. An extra 2-ft of ceiling clearance (total 10-ft) is designed over the sample position for experiments in which tall plants are vertically translated. The shielding pieces are shown schematically in Fig. 5.8 and listed with additional information in Table $\mathbf{5 . 4}$. 


\begin{tabular}{|c|c|c|c|c|c|c|c|}
\hline \multicolumn{4}{|c|}{ Walls } & \multicolumn{4}{|c|}{ Roofs } \\
\hline Shield & No. & Dim. $\left[\mathrm{ft}^{3}\right]$ & Wt. [lbs.] & Shield & No. & Dim. $\left[\mathrm{ft}^{3}\right]$ & Wt. [lbs.] \\
\hline $\mathrm{a}$ & 4 & $4 \times 6 \times 1.5$ & 40,032 & f1 & 2 & $8.5 \times 5 \times 1$ & $23,630^{\dagger}$ \\
\hline $\mathrm{b}$ & 8 & $4 \times 6 \times 1$ & 53,376 & $\mathrm{f} 2$ & 2 & $3.5 \times 5 \times 1$ & $9,730^{\dagger}$ \\
\hline $\mathrm{c}$ & 2 & $3 \times 8 \times 1$ & 13,344 & g & 2 & $8 \times 4.5 \times 1$ & 20,016 \\
\hline $\mathrm{d}$ & 7 & $4 \times 8 \times 1$ & $62,272^{*}$ & $\mathrm{~h}$ & 2 & $4 \times 5.5 \times 1$ & 12,232 \\
\hline $\mathrm{e}$ & 1 & $2.5 \times 6 \times 2$ & 8,340 & $\mathrm{~m}$ & 1 & $3.5 \times 5 \times 1$ & 4,865 \\
\hline $\mathrm{i}$ & 1 & $2 \times 3 \times 1$ & 1,668 & $\mathrm{r}$ & 1 & $4 \times 5 \times 1$ & 5,560 \\
\hline $\mathrm{j}$ & 1 & $4.5 \times 2 \times 1$ & 2,502 & & & & \\
\hline $\mathrm{k}$ & 2 & $1.5 \times 2 \times 1$ & 1,668 & & & & \\
\hline 1 & 2 & $5 \times 2 \times 1$ & 5,560 & & & & \\
\hline $\mathrm{n}$ & 1 & $4 \times 6 \times 1$ & 6,672 & & & & \\
\hline $\mathrm{p}$ & 2 & $3 \times 5.5 \times 1$ & 9,174 & & & & \\
\hline $\mathrm{q}$ & 1 & $5 \times 5.5 \times 1$ & 7,645 & & & & \\
\hline Sub-tot: & & & 212,253 & & & & 76,033 \\
\hline
\end{tabular}

Total estimated weight: $288,286 \mathrm{lbs}$.

Total estimated cost: $\$ 1,009,001$

Table 5.4. Estimations of the radiation shielding's dimensions, weight (using $278 \mathrm{lb} . / \mathrm{ft} 3$ ), and cost (using $\$ 3.50 / \mathrm{lb}$.). *Advised to subtract the weight of a $2 \times 2 \mathrm{ft}^{2}$ hole. ${ }^{\dagger}$ Advised to subtract the weight of a $3 \times 1.5 \mathrm{ft}^{2}$ hole. 


\subsubsection{CAD Drawings of the MURR-NIS}

The following CAD (computer-aided design) drawings of the MURR-NIS were designed in support of the NSF RI-1 proposal to help identify components and materials required during construction. In this regard, the exact choice, location, and dimensions of these materials are subject to change upon construction of the NIS. All sketches were made with SOLIDWORKS (2018) software at ORNL. An overview of the NIS located at E-port is shown in Fig. 5.10 along with a top-view in Fig. 5.11, side-view in Fig. 5.12, and trimetric-view in Fig. 5.13. Details of the shielding modules identified in these sketches are listed in Table $\mathbf{5 . 4}$ in the previous section.

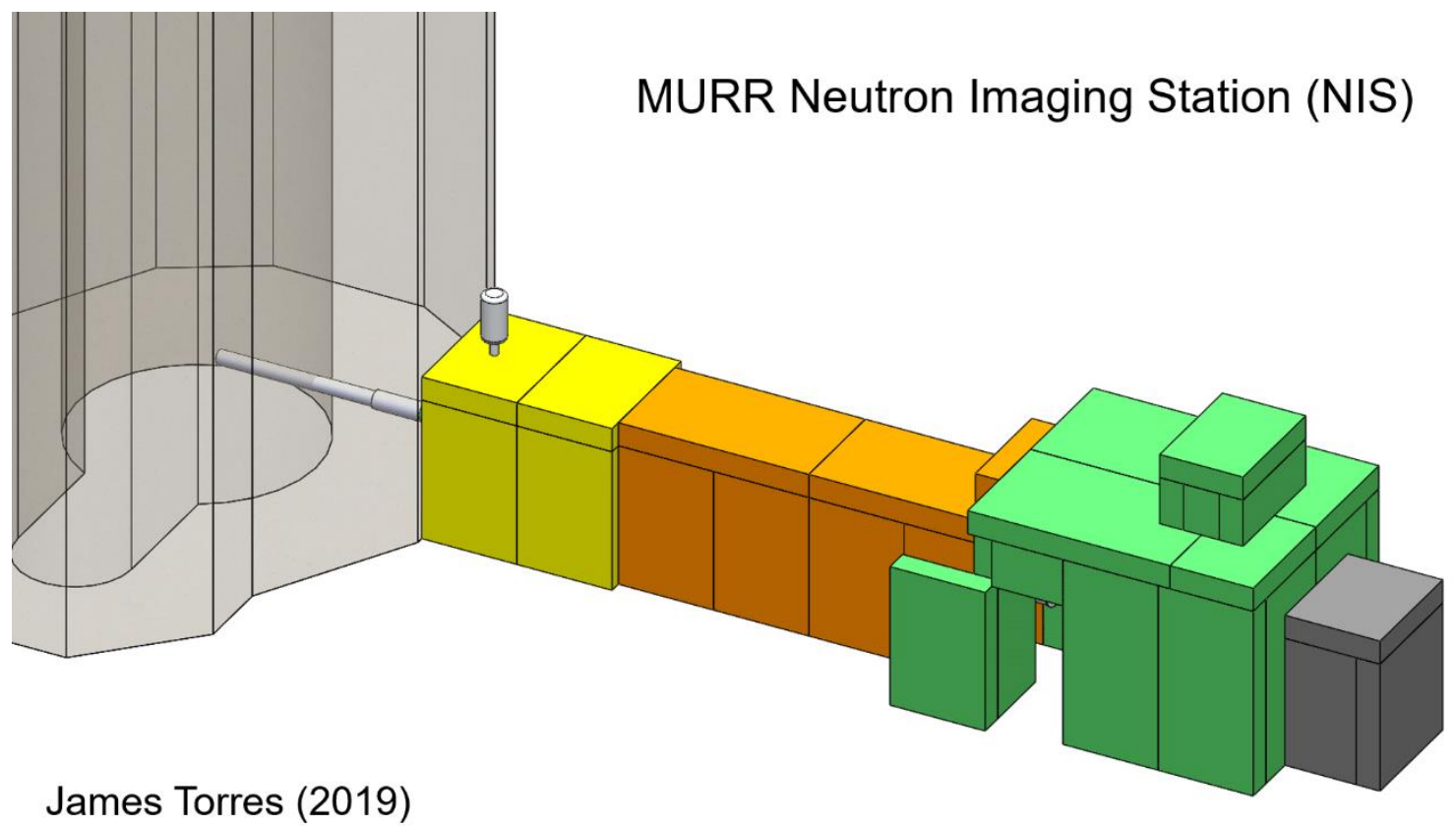

Figure 5.10. 3D sketch of the NIS conceptual design at E-port at MURR. 


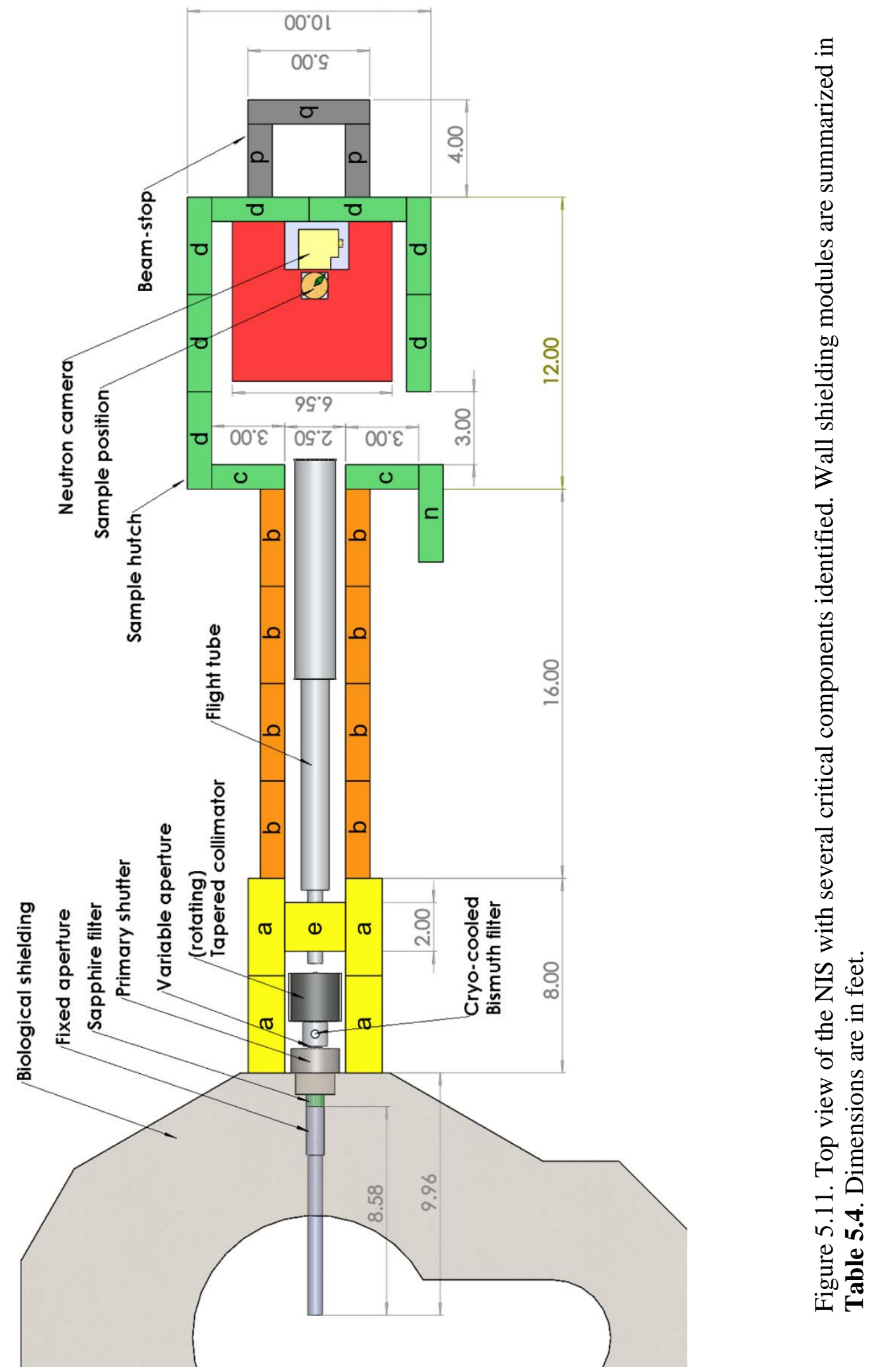




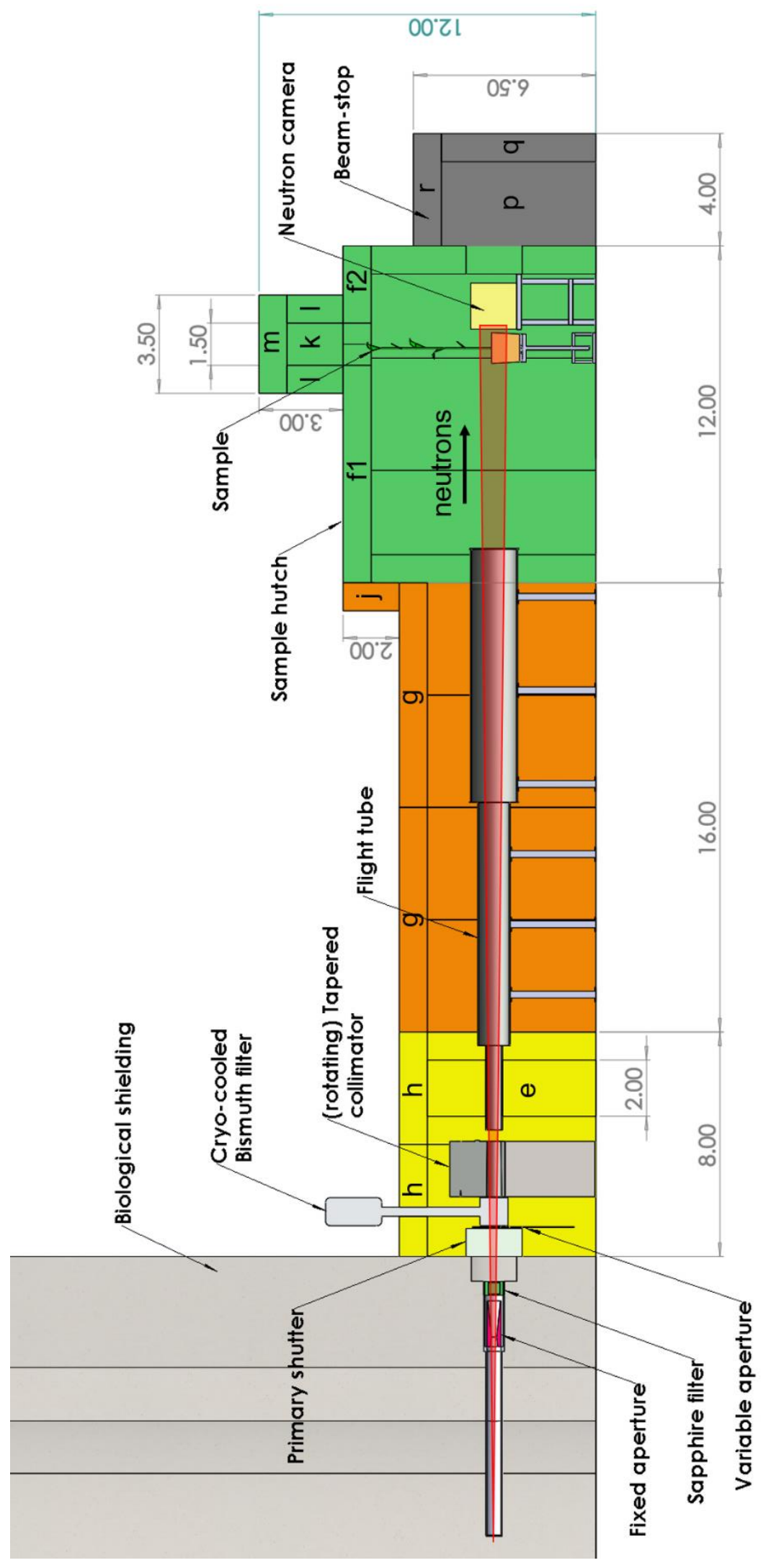

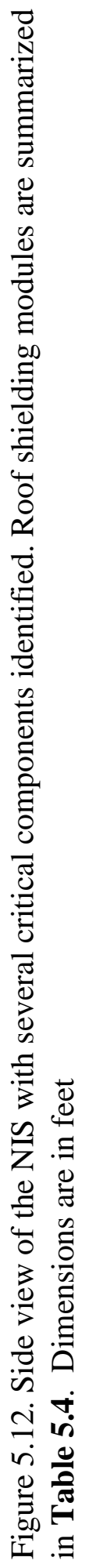




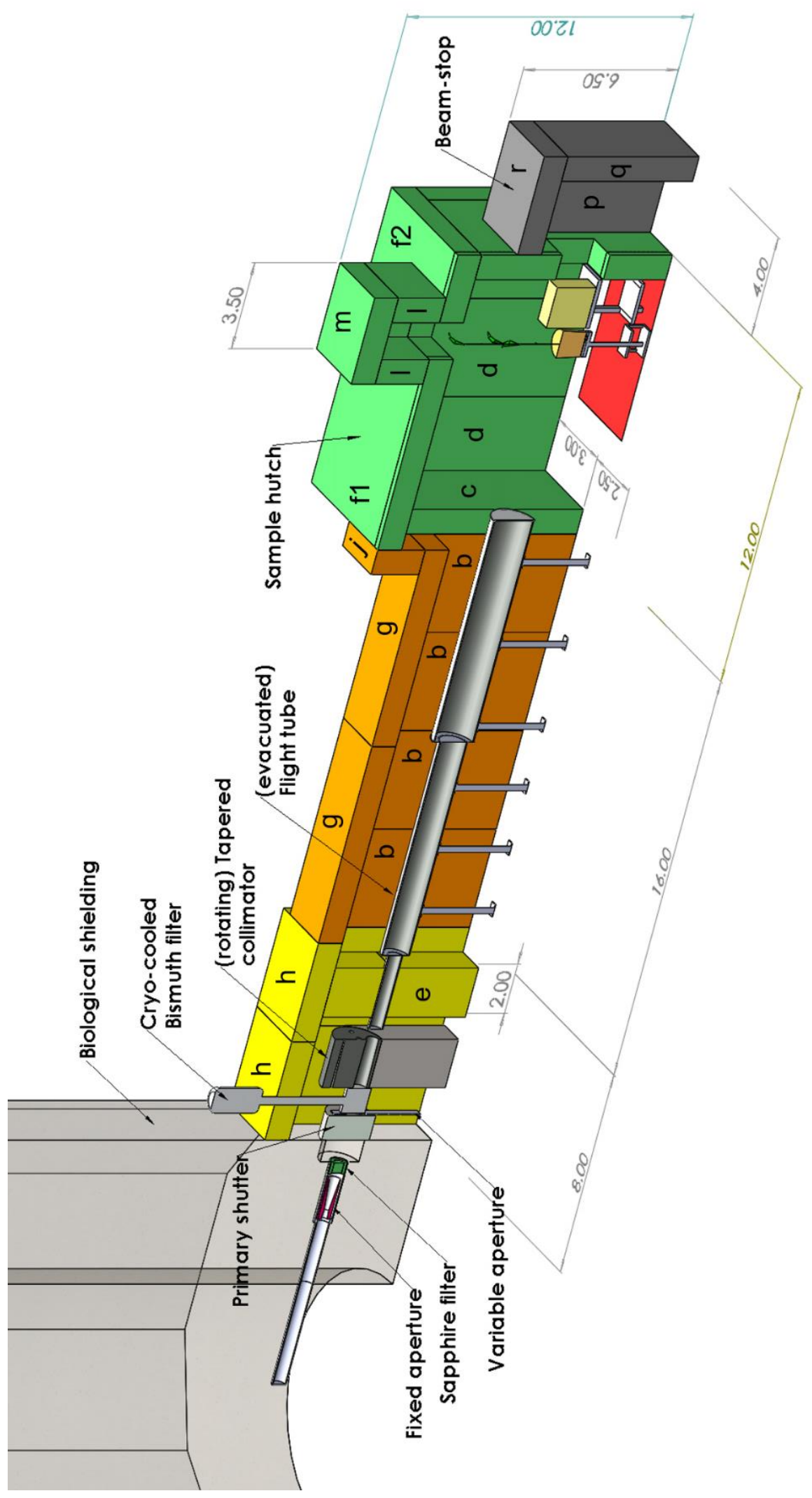

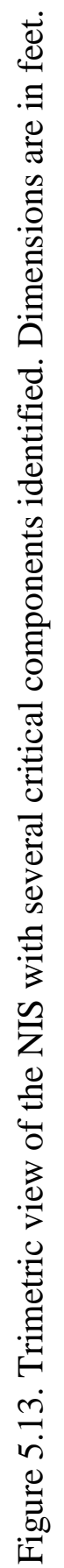




\subsubsection{McStas Neutron Ray-Tracing Computer Simulations}

Monte Carlo neutron ray-tracing simulations of the proposed beam line were carried out using the McStas 2.5 package. The goal of these simulations is to test the virtual beam line's performance, including neutron flux, divergence and image size at the detector plane using the optical components proposed in Sec. 5.2.3 (see Fig. 5.7). The script used to simulate the $L / D=400$ setting is given in Appendix $\mathbf{D}$.

In the first round of simulations, we used a simple source that is flat in neutron intensity vs. energy to determine the size and divergence of the beam transmitted through two apertures: a fixed aperture $\left(D_{0}=11.23 \mathrm{~cm}\right)$ and a variable aperture $(D=2.5 \mathrm{~cm}$ for $L / D=400$ ) placed $2.15 \mathrm{~m}$ and $3.34 \mathrm{~m}$ from the source, respectively. Two positionsensitive detectors (PSD) were placed at the calculated detector and beam-stop planes located $13.34 \mathrm{~m}$ and $14.65 \mathrm{~m}$ from the source, respectively. To attenuate fast neutrons, we added a community-developed sapphire filter component, $2.62 \mathrm{~m}$ from the source. Unfortunately, a bismuth filter was not available in the current (2.5) McStas distribution; Therefore, we modelled the total scattering cross section of bismuth from a polynomial function developed by Freund [195] to experimental data of neutron attenuation coefficient $\Sigma$ vs. neutron wavelength (in $\mathrm{nm}$ ),

$$
\Sigma(\lambda)=A \lambda+C\left\{1-\exp \left[-\left(\frac{B}{\lambda^{2}}+\frac{D}{\lambda^{4}}\right)\right]\right\}
$$

where $A, B, C$, and $D$ are the fitted parameters used to model the transmission of bismuth. The total cross sections for sapphire and bismuth were reproduced from Refs. [195] and [204] in Fig. 5.14 and Fig. 5.15, respectively. The fit of Eq. (5.4) to bismuth is shown in

Fig. 5.16. As discussed in Sec. 5.2.3 (also see Figs. 5.14 and 5.15), it is more 
advantageous to cryogenically cool the bismuth filter compared to sapphire as this leads to a substantial increase in neutron flux, especially in the thermal neutron energy range.

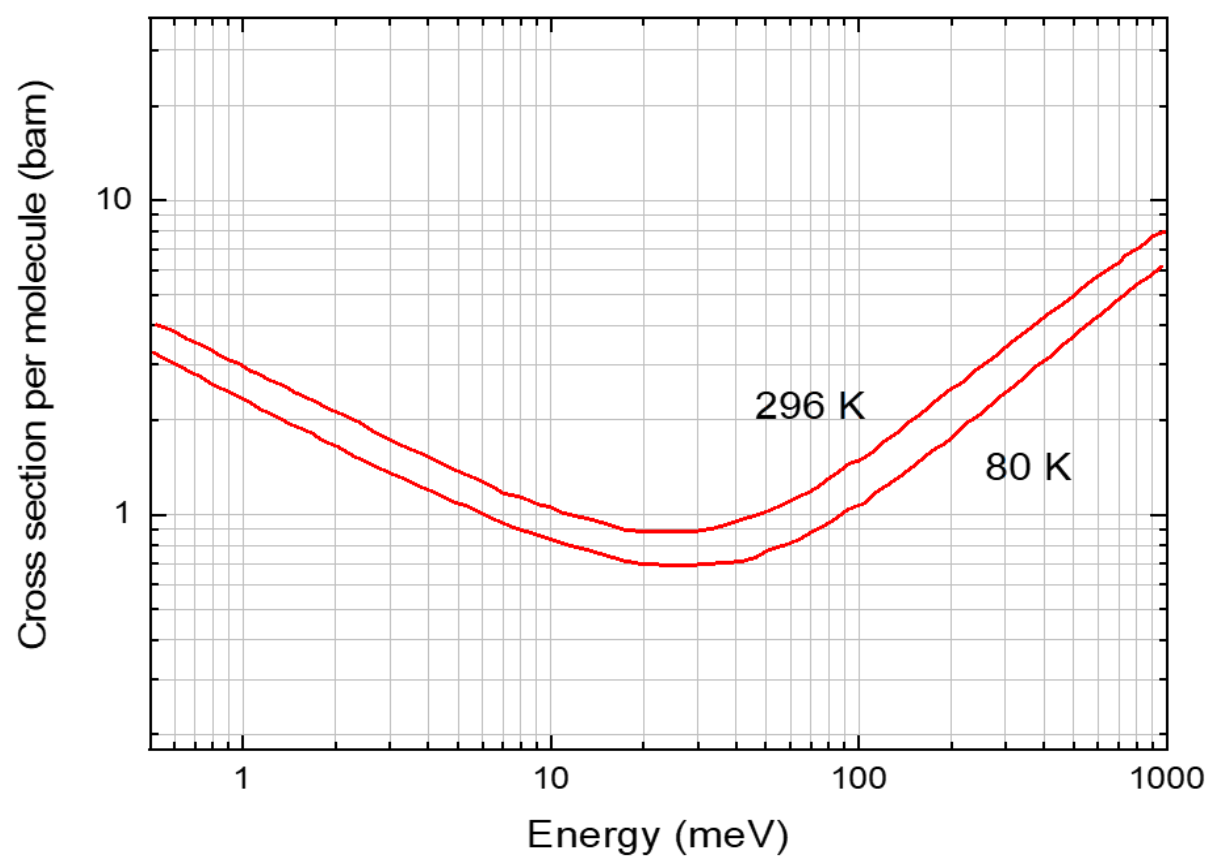

Figure 5.14. Total (calculated) neutron cross section of sapphire at temperatures of $296 \mathrm{~K}$ and $80 \mathrm{~K}$ vs. neutron energy. Reproduced from Ref. [195].

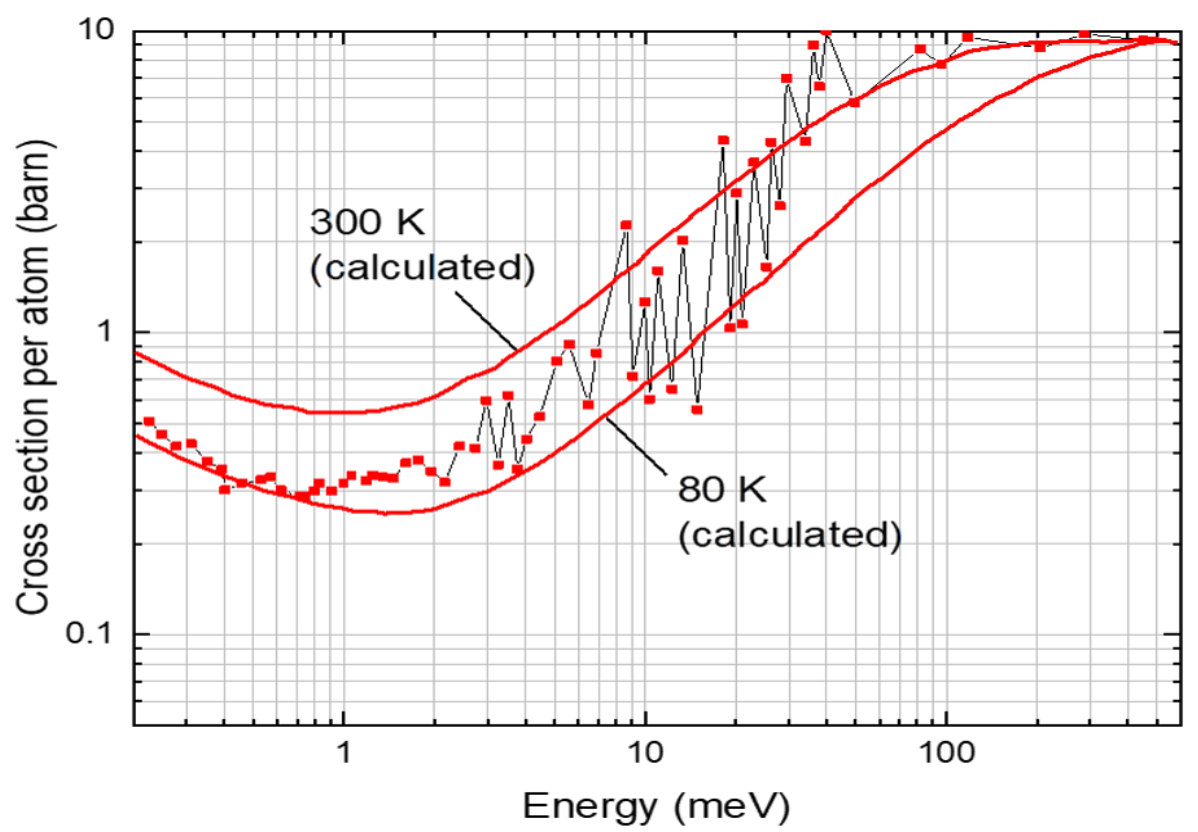

Figure 5.15. Experimental data for the total neutron cross section of bismuth at temperature of $100 \mathrm{~K}$ vs. neutron energy. Solid curves correspond to calculated total cross sections at $300 \mathrm{~K}$ and $80 \mathrm{~K}$. Reproduced from Ref. [195]. 


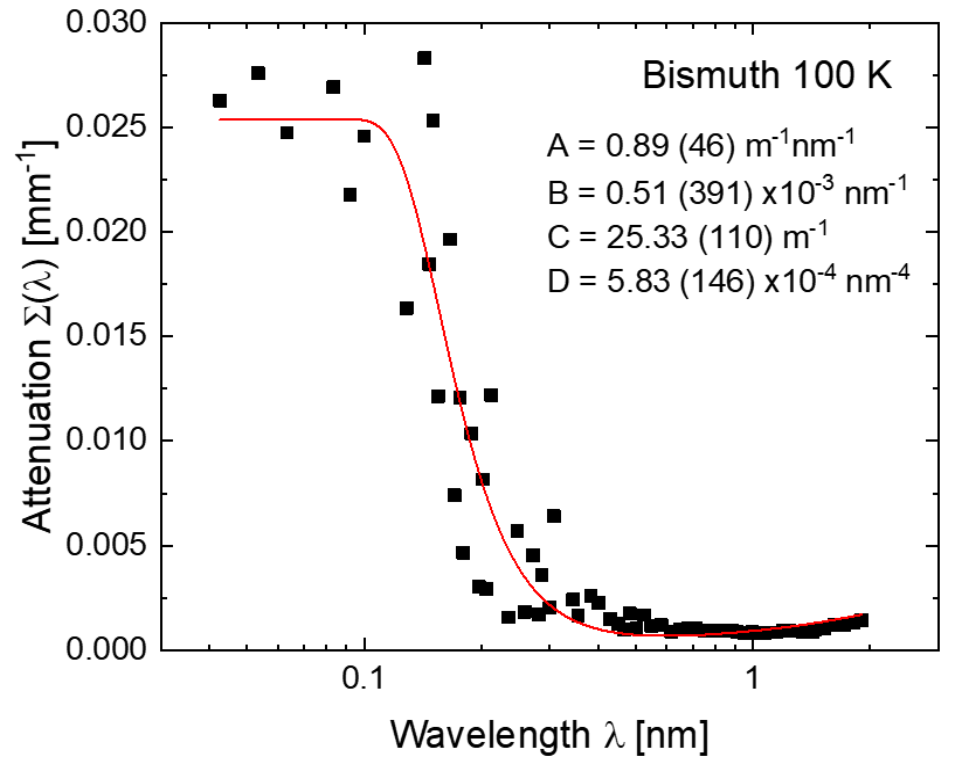

Figure 5.16. Semi-log plot of the attenuation coefficient $\Sigma$ of Bi at $100 \mathrm{~K}$ vs. neutron wavelength, derived by multiplying the total cross section (see Fig. 5.15 and Ref. [195]) by the number density $N$ for bismuth $\left(2.82\right.$ barn $\left.^{-1} \mathrm{~m}^{-1}\right)$. The best fit to the data is shown by the red curve.

A summary of these initial simulations is given in Fig. 5.17, which includes the total neutron count in the PSDs at the (a) detector and (b) beam-stop positions as well as neutron counts in (c) a wavelength-dependent intensity monitor and (d) an angular divergence monitor where the latter two are located at the detector position. In Fig. 5.17a, the umbra diameter measures about $36 \mathrm{~cm}$, in agreement with the calculated $\sim 35.5 \mathrm{~cm}$ diameter in Table 5.2. From Fig. 5.17b, the beam-stop must have a minimum diameter of $60 \mathrm{~cm}$ to capture the size of the penumbra. Fast-neutron attenuation from the initially flat source is demonstrated in Fig. 5.17c, which also demonstrates the cost in neutron intensity for using filters. The total beam divergence $\alpha$ follows as,

$$
\alpha=\operatorname{atan}\left(\frac{D}{2 L}\right)
$$

which for $L / D=400$ is $\sim 0.072^{\circ}$ and equivalent to the simulated beam divergence in Fig. 5.17d. A summary of these simulations for all $L / D$ settings is given in Table $\mathbf{5 . 5}$. 


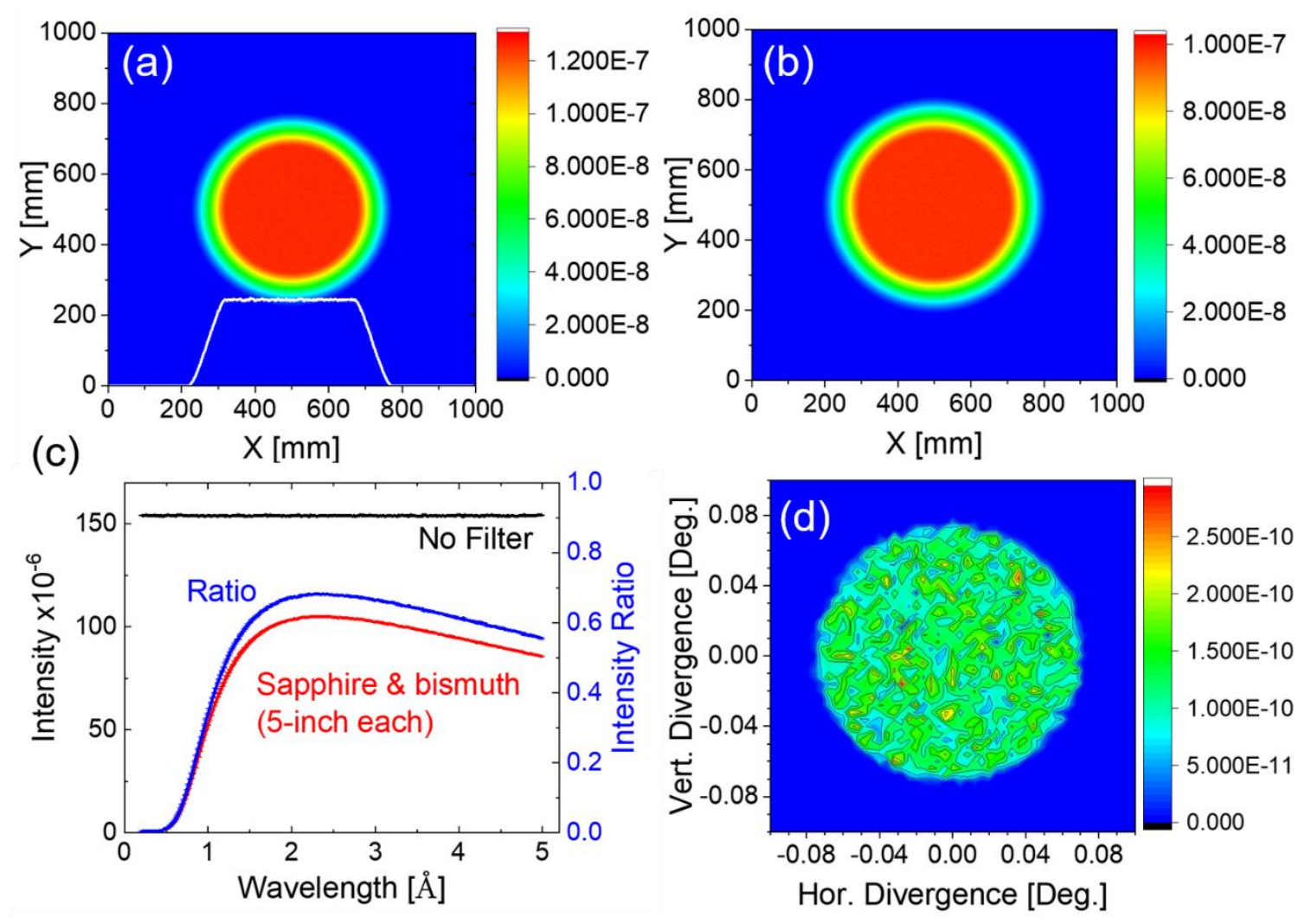

Figure 5.17. Simulation results of the NIS for the L/D = 400 setting. $1 \times 1 \mathrm{~m}^{2}$ PSDs recorded neutron intensity at the (a) detector and (b) beam-stop locations. A line scan through the beam cross section is shown in (a) with an umbra diameter of $\sim 36 \mathrm{~cm}$. (c) Wavelength-dependent intensity integrated over the $1 \times 1 \mathrm{~m}^{2}$ detector: without sapphire and bismuth filters (black), with 5-inch-thick filters (red), and the ratio filter-to-no filter (blue) to demonstrate the penalty of using filters. (d) $1 \times 1 \mathrm{~mm}^{2}$ beam-divergence monitor at the detector plane showing $\sim 0.072^{\circ}$ divergence.

\begin{tabular}{ccccccc} 
Aperture & $\boldsymbol{L} / \boldsymbol{D}$ & $\begin{array}{c}\text { Umbra } \\
{[\mathrm{cm}]}\end{array}$ & $\begin{array}{c}\text { Penumbra } \\
{[\mathrm{cm}]}\end{array}$ & $\begin{array}{c}\text { Hor. Div. } \\
{[\text { Deg.] }}\end{array}$ & $\begin{array}{c}\text { Vert. Div. } \\
{[\text { Deg.] }}\end{array}$ & Intensity $^{*}$ \\
\hline Fixed & 100 & 9 & 98 & 0.29 & 0.29 & $32 \%$ \\
\hline \multirow{5}{*}{ Variable } & 200 & 26 & 72 & 0.14 & 0.14 & $4 \%$ \\
& 400 & 36 & 62 & 0.072 & 0.072 & $1 \%$ \\
& 1600 & 40 & 56 & 0.036 & 0.036 & $0.3 \%$ \\
& 2000 & 43 & 54 & 0.018 & 0.018 & $0.07 \%$ \\
& 42 & 52 & 0.014 & 0.014 & $0.04 \%$
\end{tabular}

Table 5.5. Results of neutron ray-tracing simulations. Approximate diameters of the umbra and penumbra precede the use of collimation (e.g., beam-scrapers). * Measured at the detector plane relative to the intensity at the fixed aperture. 


\section{Appendix A - Samples and Sample Cell Used for Neutron Scattering}

Sample 1-80 CuO-coated [20] copper foil disks, interlaid with 79 annular aluminum foil rings, and sealed with $120 \mu \mathrm{l}$ of $\mathrm{H}_{2} \mathrm{O}$ under helium atmosphere. Prior to sealing, the foils were heated in air at $55-60{ }^{\circ} \mathrm{C}$ for about 13 hours to remove excess water from their surfaces. The sample was measured on the HFBS in May of 2015 during a proof-ofprinciple study with cooling and heating scans shown in Fig. A.1.

Sample 2 - $100 \mathrm{CuO}$-coated [56] copper foil disks sealed with $60 \mu \mathrm{l}$ of $\mathrm{H}_{2} \mathrm{O}$ under helium atmosphere. Prior to sealing, the foils were heated in air at $\sim 55{ }^{\circ} \mathrm{C}$ for about 3 days to remove excess water from their surfaces. An aluminum spacer was used to ensure the foils were in the $~ 30$-mm-tall neutron beam. Measured on the HFBS in September of 2016 in collecting elastic scans (Figs. 3.3a, 3.4, 4.5, 4.10, B.2a) and quasielastic spectra (Figs. 4.6, 4.8).

Sample 3-100 CuO-coated [56] copper foil disks sealed with $10 \mu \mathrm{l}$ of $\mathrm{H}_{2} \mathrm{O}$ under helium atmosphere. Prior to sealing, the foils were heated in air at $\sim 55^{\circ} \mathrm{C}$ for about 3 days to remove excess water from their surfaces. An aluminum spacer was used to ensure the foils were in the $\sim 30-\mathrm{mm}$-tall neutron beam. Measured on the HFBS in September of 2016 in collecting elastic scans (Figs. 3.3b, 3.4, 4.5, 4.10, B.2b). 
Sample 4 - 100 bare (untreated) copper foil disks sealed with $60 \mu \mathrm{l}$ of $\mathrm{H}_{2} \mathrm{O}$ under helium atmosphere. Measured on the HFBS in September of 2016 in collecting elastic scans (Figs. 3.3c, 4.5, B.2c).

Sample 5 - 100 CuO-coated [56] copper foil disks sealed without any added $\mathrm{D}_{2} \mathrm{O}$, i.e., "dry," under helium atmosphere. Measured on the MURR PSD-diffractometer in collecting background diffraction patterns (Figs. 3.6, 3.7d, C.4) prior to hydrating with $\mathrm{D}_{2} \mathrm{O}$.

Sample 6 - 100 CuO-coated [56] copper foil disks (previously Sample 5) sealed with 120 $\mu 1$ of $\mathrm{D}_{2} \mathrm{O}$ under helium atmosphere. Measured on the MURR PSD-diffractometer in collecting diffraction patterns as a function of temperature (Figs. 3.7c, C.1, C.4). Sample checked for leaks via leak detector at the MU Physics Machine Shop.

Sample 7 - 100 CuO-coated [56] copper foil disks (previously Sample 6) sealed with 240 $\mu 1$ of $\mathrm{D}_{2} \mathrm{O}$ under helium atmosphere. Measured on the MURR PSD-diffractometer in collecting diffraction patterns as a function of temperature (Figs. 3.7b, 3.8, C.2-C.8).

Sample 8 - 100 bare copper foil disks sealed with $120 \mu 1$ of $\mathrm{D}_{2} \mathrm{O}$ under helium atmosphere. Measured on the MURR PSD-diffractometer in collecting diffraction patterns as a function of temperature (Figs. 3.7a, C.4).

Sample 9-100 CuO-coated [56] copper foil disks (1-cm-dia.) sealed with $6 \mu 1$ of $\mathrm{H}_{2} \mathrm{O}$ under helium atmosphere. Measured on the cold-neutron chopper spectrometer (CNCS) at the ORNL Spallation Neutron Source in collecting quasielastic spectra at $\sim 293 \mathrm{~K}$.

Sample 10 - $100 \mathrm{CuO-coated} \mathrm{[56]} \mathrm{copper} \mathrm{foil} \mathrm{disks} \mathrm{(1-cm-dia.)} \mathrm{sealed} \mathrm{without} \mathrm{any} \mathrm{added}$ $\mathrm{H}_{2} \mathrm{O}$, i.e., "dry," under helium atmosphere. Measured on the cold-neutron chopper 
spectrometer (CNCS) at ORNL in collecting quasielastic spectra at room-temperature ( 293 K).

Sample 11 - $100 \mathrm{CuO}$-coated [56] copper foil disks sealed with $60 \mu 1$ of $\mathrm{H}_{2} \mathrm{O}$ under helium atmosphere. Measured on $\mathrm{BaSiS}$ in collecting quasielastic spectra in the temperature range $230-270 \mathrm{~K}$ with a resolution scan at $100 \mathrm{~K}$ (Figs. 4.9-4.16) This sample was also measured on the VISION spectrometer at ORNL-SNS in May of 2018 in collecting vibrational spectra (Figs. 4.2-4.4).

Sample 12 - $100 \mathrm{CuO}$-coated [56] copper foil disks sealed with $10 \mu \mathrm{l}$ of $\mathrm{H}_{2} \mathrm{O}$ under helium atmosphere. Measured on $\mathrm{BaSiS}$ in collecting quasielastic spectra in the temperature range 230-270 $\mathrm{K}$ with a resolution scan at $100 \mathrm{~K}$ (Figs. 4.10-4.13, 4.154.16). This sample was also measured on VISION in May of 2018 in collecting vibrational spectra (Figs. 4.2-4.4).

Sample 13 - 100 bare copper foil disks sealed with $10 \mu 1$ of $\mathrm{H}_{2} \mathrm{O}$ under helium atmosphere. Measured on BASIS in collecting quasielastic spectra at sample temperatures of $260 \mathrm{~K}$ and $270 \mathrm{~K}$ with a resolution scan at $100 \mathrm{~K}$. This sample was also measured on VISION starting in May 2018 in collecting vibrational spectra at a temperature of $5 \mathrm{~K}$.

Sample 14 - 100 bare copper foil disks sealed without any added $\mathrm{H}_{2} \mathrm{O}$ under helium atmosphere. Measured on VISION in May of 2018 at a temperature of $5 \mathrm{~K}$ in collecting background vibrational spectra contributed by the copper foil substrates at a temperature of $5 \mathrm{~K}$ (Figs. 4.2, 4.3). 
Sample 15 - Previously Sample 14 now oxidized (with assistance from Luke Daemen) to form $\mathrm{CuO}$-coated [56] copper foil disks sealed without any added $\mathrm{H}_{2} \mathrm{O}$ under helium atmosphere. The sample was dried in a vacuum flange for 24 hours without applying heat. Measured on VISION in May of 2018 in collecting background vibrational spectra contributed by the $\mathrm{CuO}$ coating and copper foil substrates at a temperature of $5 \mathrm{~K}$ (Figs. 4.2, 4.3).

Sample 16 - Previously Sample 11 (wet $\mathrm{CuO}$ sample measured on BaSiS) now opened and dried in vacuum at $\sim 100{ }^{\circ} \mathrm{C}$ for 24 hours to remove excess water. Measured on VISION in May of 2018 on heating from a sample temperature of $5 \mathrm{~K}$ to $50 \mathrm{~K}, 100 \mathrm{~K}$, $150 \mathrm{~K}, 200 \mathrm{~K}$ and then cooled to $150 \mathrm{~K}, 100 \mathrm{~K}, 50 \mathrm{~K}$, and $5 \mathrm{~K}$. Used to compare with Sample 15.

Sample 17 - Previously Sample 16 now opened and hydrated with $60 \mu$ of $\mathrm{H}_{2} \mathrm{O}$. Measured on VISION in May of 2018 on heating from a sample temperature of 5 K (Fig. 4.4) to $50 \mathrm{~K}, 100 \mathrm{~K}, 200 \mathrm{~K}$, and $250 \mathrm{~K}$ and then cooled to $200 \mathrm{~K}, 150 \mathrm{~K}, 100 \mathrm{~K}, 50 \mathrm{~K}$, and 5 K. Used to compare with Sample 11 in Fig. 4.4.

Sample 18 - Previously Sample 15 now opened, dried in vacuum at $200{ }^{\circ} \mathrm{C}$ (max. temperature of the vacuum-oven) for about 54 hours to remove excess and adsorbed water, based on the results from TG measurements. Measured on VISION in August of 2018 at a sample temperature of $5 \mathrm{~K}$ to compare with Samples 15 and 16.

Sample 19 - Previously Sample 18 now opened and hydrated with $60 \mu 1$ of water (in air). After sealing, the sample was heated at $55^{\circ} \mathrm{C}$ for $\sim 18$ hours to evaporate and redistribute water inside. Measured on VISION in August of 2018 at a sample temperature of $5 \mathrm{~K}$. 
Sample 20 - Previously Sample 19 opened and hydrated with an additional $60 \mu 1$ of water (in air), totaling $120 \mu \mathrm{l}$ of $\mathrm{H}_{2} \mathrm{O}$. After sealing, the sample was heated at $55^{\circ} \mathrm{C}$ for $\sim 19$ hours to evaporate and redistribute water inside. Measured on VISION in August of 2018 at a sample temperature of $5 \mathrm{~K}$.

Sample 21 - Previously Sample 20 opened and hydrated with an additional $60 \mu 1$ of water (in air), totaling $180 \mu \mathrm{l}$ of $\mathrm{H}_{2} \mathrm{O}$. After sealing, the sample was heated at $55^{\circ} \mathrm{C}$ for $\sim 4$ hours to evaporate and redistribute water inside. Measured on VISION in August of 2018 at a sample temperature of $5 \mathrm{~K}$.

Sample 22 - Previously Sample 21 opened and hydrated with an additional $60 \mu 1$ of water (in air), totaling $240 \mu \mathrm{l}$ of $\mathrm{H}_{2} \mathrm{O}$. (Unknown heating time after sealing - performed by L. Daemen). Measured on VISION at a sample temperature of $5 \mathrm{~K}$.

\begin{tabular}{|c|c|c|c|c|}
\hline $\begin{array}{l}\text { Sample } \\
\text { No. }\end{array}$ & $\begin{array}{c}\text { Surface } \\
\text { Composition }\end{array}$ & $\begin{array}{c}\text { Hydration } \\
{[\mu 1]}\end{array}$ & $\begin{array}{c}\text { Time of } \\
\text { Measurement }\end{array}$ & Appears in Figure(s) \\
\hline 1 & $\mathrm{CuO}$ & 120 & May 2015 & A.1 \\
\hline 2 & $\mathrm{CuO}$ & 60 & September 2016 & $\begin{array}{c}\text { 3.3a, } 3.4,4.5,4.6,4.8 \\
\text { 4.10, B.2a }\end{array}$ \\
\hline 3 & $\mathrm{CuO}$ & 10 & September 2016 & $3.3 \mathrm{~b}, 3.4,4.5,4.10$, B.2b \\
\hline 4 & $\mathrm{Cu}_{2} \mathrm{O} / \mathrm{Cu}$ & 60 & September 2016 & $3.3 \mathrm{c}, 4.5$, B.2c \\
\hline 5 & $\mathrm{CuO}$ & 0 & November 2016 & 3.5, 3.7d, C.4 \\
\hline 6 & $\mathrm{CuO}$ & 120 & Nov.-Dec. 2016 & 3.7c, C.1, C.4 \\
\hline 7 & $\mathrm{CuO}$ & 240 & Jan.-Feb. 2017 & 3.7b, 3.8, C.2-C.8 \\
\hline 8 & $\mathrm{Cu}_{2} \mathrm{O} / \mathrm{Cu}$ & 120 & July-Aug. 2017 & 3.7a, C.4 \\
\hline 9 & $\mathrm{CuO}$ & 6 & September 2017 & N/A \\
\hline 10 & $\mathrm{CuO}$ & 0 & September 2017 & N/A \\
\hline 11 & $\mathrm{CuO}$ & 60 & Dec. '17/May '18 & $4.2-4.4,4.9-4.16$ \\
\hline 12 & $\mathrm{CuO}$ & 10 & Dec. '17/May '18 & $\begin{array}{c}4.2-4.4,4.10-4.13,4.15 \text {, } \\
4.16\end{array}$ \\
\hline 13 & $\mathrm{Cu}_{2} \mathrm{O} / \mathrm{Cu}$ & 10 & Dec. '17/May '18 & N/A \\
\hline 14 & $\mathrm{Cu}_{2} \mathrm{O} / \mathrm{Cu}$ & 0 & May 2018 & $4.2,4.3$ \\
\hline
\end{tabular}




\begin{tabular}{|l|l|c|c|c|}
\hline 15 & $\mathrm{CuO}$ & 0 & May 2018 & $\mathbf{4 . 2}, \mathbf{4 . 3}$ \\
\hline 16 & $\mathrm{CuO}$ & 0 & May 2018 & N/A \\
\hline 17 & $\mathrm{CuO}$ & 60 & May 2018 & $\mathbf{4 . 4}$ \\
\hline 18 & $\mathrm{CuO}$ & 0 & August 2018 & N/A \\
\hline 19 & $\mathrm{CuO}$ & 60 & August 2018 & N/A \\
\hline 20 & $\mathrm{CuO}$ & 120 & August 2018 & N/A \\
\hline 21 & $\mathrm{CuO}$ & 180 & August 2018 & N/A \\
\hline 22 & $\mathrm{CuO}$ & 240 & August 2018 & N/A \\
\hline
\end{tabular}

Table A.1. List of samples, their composition, and the period in which they were used in neutron scattering experiments, between May of 2015 and August of 2018. The rightmost column shows where to find the data corresponding to each sample.

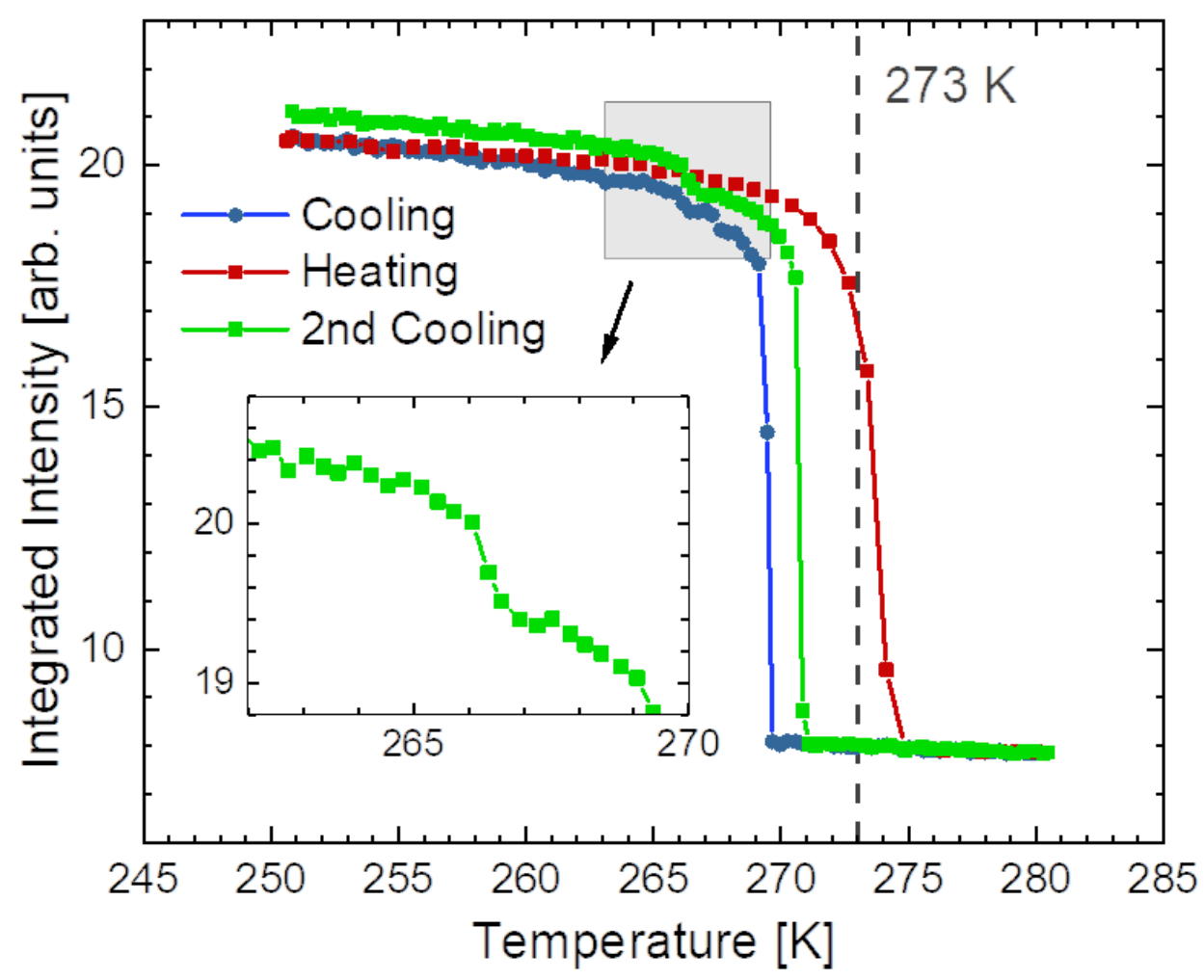

Figure A.1. Temperature-dependent elastic neutron scattering scans of a CuO-coated sample with $120 \mu \mathrm{l}$ of water added that hydrates $80 \mathrm{CuO}$-coated [20] copper foil disks (Sample \#1) as measured on the HFBS at the NIST Center for Neutron Research in May 2015. The elastic intensity was summed over all 16 detectors, which span a wave vector transfer $(Q)$ range of $0.25-1.75 \AA^{-1}$. The vertical dashed line is drawn at the bulk melting point of water $\left(\mathrm{H}_{2} \mathrm{O}\right)$ at $273 \mathrm{~K}$. The inset shows an inflection in the elastic intensity of the $2^{\text {nd }}$ cooling scan that we interpret as a $2^{\text {nd }}$ freezing transition at $\sim 266.5 \mathrm{~K}$ of interfacial water which occurs after the initial freezing transition at $\sim 270.5 \mathrm{~K}$. 


\section{Sample Cell}

To enhance the scattering from the interfacial water on the surfaces of our copper foil samples, a large sample volume was desired. Samples 1-8 and 11-22 were loaded into cylindrical aluminum (6061-T6) cans, the main body of which is shown schematically in Fig. A.2, that was fabricated at the University of Missouri Physics Machine Shop. The total available inner volume is about $75 \mathrm{~cm}^{3}$, which is used to house a stack of 100 copper foil disks, each $\sim 5 \mathrm{~cm}$ in diameter and about $12.7 \mu \mathrm{m}$ thick. Bends and folds from handling the foils during sample preparation effectively increases the height of the 100stack, allowing the foils to fill the cell volume with a positive pressure applied by the lid. In some cases, a flexible aluminum spacer (not shown) is added to the cell in order to ensure the copper foils fill the neutron beam cross section. The cell is sealed with indium wire (Alfa Aesar, 99.99\% purity) of diameter 0.06 inches $(\sim 1.5 \mathrm{~mm})$. 

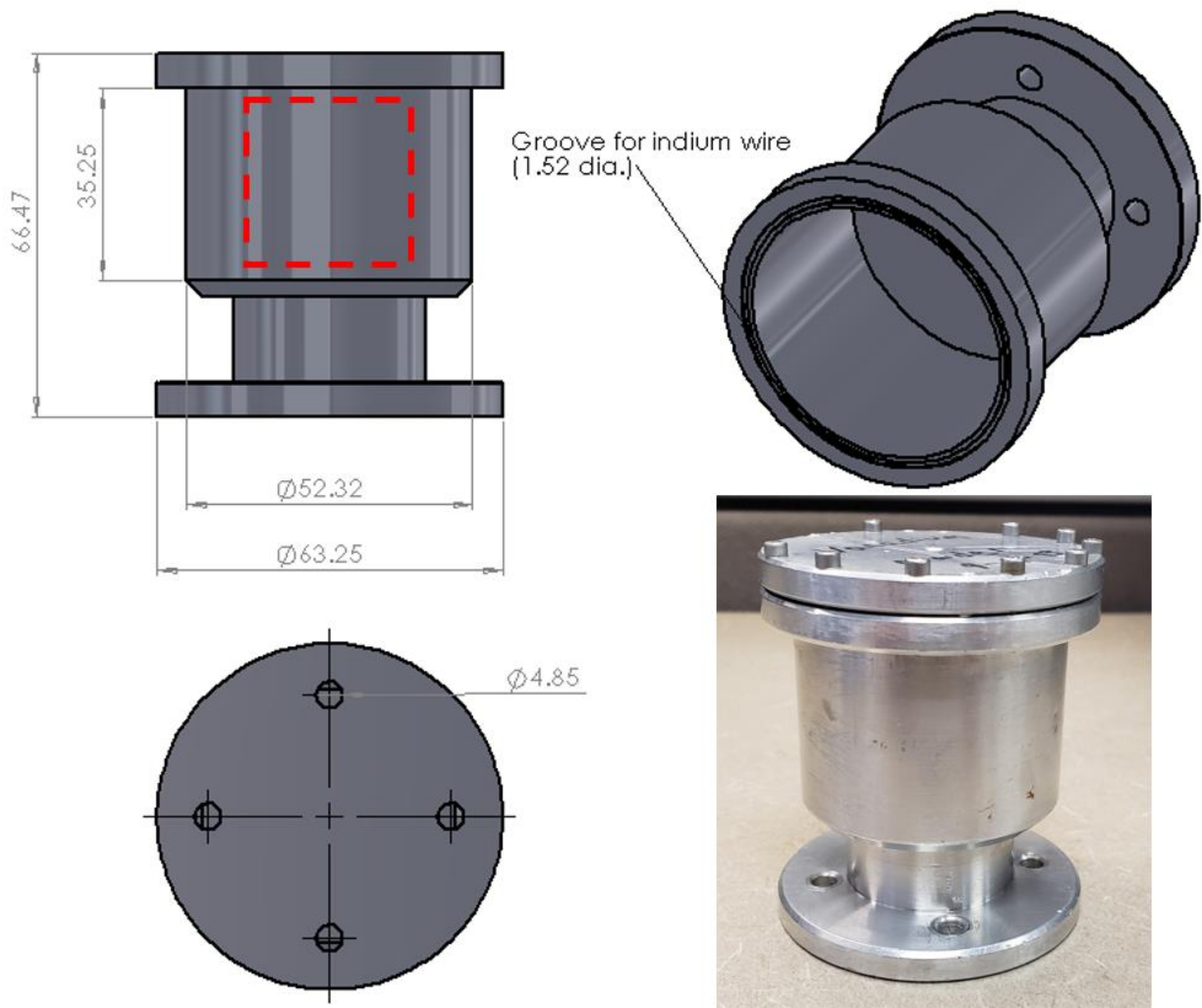

Figure A.2. 3D schematic of the main body of the sample cell used for neutron scattering experiments: (top left) side view, (bottom left) bottom view, (top right) trimetric view, and (bottom right) photo of a sealed cell. The red dashed box represents a $30 \times 30 \mathrm{~mm}^{2}$ cross section of the neutron beam approximately equal to the beam size on the HFBS and BASIS that is incident on the thin $(\sim 508 \mu \mathrm{m})$ wall of the aluminum cell. All dimensions are in millimeters. 


\section{Appendix B - Estimating the Amount of}

\section{Frozen and/or Immobile Water in HFBS}

\section{Elastic Scans}

This appendix provides a rough estimate of the number of water molecules that contribute to the relative change in the intensity of elastically scattered neutrons from CuO-coated and bare- $\mathrm{Cu}$ foil samples hydrated with $\mathrm{H}_{2} \mathrm{O}$ as measured on the High-Flux Backscattering Spectrometer (HFBS) in September of 2016. These estimations are based on a calibration against an alkane standard sample, which contains a known number of $\mathrm{H}-$ atoms that become immobile on the experimental time scale. A scale factor is defined to account for the difference in the incoherent scattering from the different substrates $(\mathrm{Cu}$ vs. Si). These details are given in the first section. In the second section, we estimate $\sim 3.4$ mmol of water freezing on bare $\mathrm{Cu}$ foils. The equivalent thickness of the (theoretical) water layer is $\sim 1,500 \AA$ on both sides of the $100 \mathrm{Cu}$ foils, assuming the bulk density of water and perfectly flat $\mathrm{Cu}$ surfaces. A similar treatment yields $\sim 2,000 \AA$ and $\sim 1,000 \AA$ water film thicknesses for the $\mathrm{CuO}$ samples hydrated with $60 \mu \mathrm{l}$ and $10 \mu \mathrm{lof} \mathrm{H}_{2} \mathrm{O}$, respectively. 


\section{B.1 Alkane Standard}

In principle, it is possible to quantify the number of $\mathrm{H}$ atoms in the HFBS scattering volume by calibration against a well-characterized standard under equivalent experimental conditions. Below, we summarize the experiment and analysis of an "alkane standard" sample whose elastic intensity was measured previously on the HFBS [167]. The sample was prepared by dip-coating 100 electronic-grade silicon wafers, each measuring $5 \mathrm{~cm}$ in diameter and 300- $\mu \mathrm{m}$-thick (approx. $3.04 \times 10^{24} \mathrm{Si}$ atoms), in a solution consisting of $\mathrm{C}_{32} \mathrm{H}_{66}$ (herein designated as $\mathrm{C}_{32}$ ) nanoparticles suspended in heptane. After sealing in an aluminum sample can (see Fig. A.2) under argon atmosphere, the sample was heated above the bulk melting point of monoclinic $\mathrm{C}_{32}$ (341 K) and then cooled to $318 \mathrm{~K}$. Subsequent AFM measurements confirmed the $\mathrm{C}_{32}$ molecules were oriented perpendicular to the Si surface, each molecule occupied an average area of $18.9 \AA^{2}$, and together they formed mesa-like agglomerates.

In Fig. B.1, we reproduced from Ref. [167] a plot of the elastic scattering intensity summed over all detectors vs. temperature as measured on the HFBS at NIST for a sample of 10 monolayers of $\mathrm{C}_{32}$ deposited on $100 \mathrm{Si}$ wafers. The temperaturedependent elastic intensity $I(T)$ is the sum of the scattering from all elements/isotopes of the sample $(\mathrm{Si}, \mathrm{H}, \mathrm{O}, \mathrm{C})$ and can be expressed as,

$$
I(T)=I_{0} \sum_{i} N_{i} \sigma_{i}
$$

Here, $I_{0}$ is a scalar quantity which is sensitive to instrument settings; and, $N_{i}$ and $\sigma_{i}$ are the number and total scattering cross section of the $i^{t h}$ atom. The coherent $\left(\sigma_{c o h}\right)$ and 
incoherent $\left(\sigma_{i n c}\right)$ scattering cross sections of the known elements of the alkane standard are given in Table B.1. From this table, it is reasonable to conclude that the total scattering is dominated by the incoherent scattering from hydrogen, which is at least four orders of magnitude higher than the incoherent scattering from the other elements.

Therefore, Eq. (B.1) simplifies to,

$$
I(T)=I_{0} \sum_{i} N_{H} \sigma_{\text {inc }}(H)
$$

\begin{tabular}{ccc} 
Element & $\boldsymbol{\sigma}_{\boldsymbol{c o h}}[\mathrm{barn}]$ & $\boldsymbol{\sigma}_{\boldsymbol{i n c}}[\mathrm{barn}]$ \\
\hline $\mathrm{H}$ & 1.7568 & 80.26 \\
$\mathrm{C}$ & 5.551 & 0.001 \\
$\mathrm{O}$ & 4.232 & 0.0008 \\
$\mathrm{Si}$ & 2.163 & 0.004
\end{tabular}

Table B.1. Coherent $\left(\sigma_{c o h}\right)$ and incoherent scattering $\left(\sigma_{i n c}\right)$ cross sections of the elements in the alkane standard sample from Ref. [95].

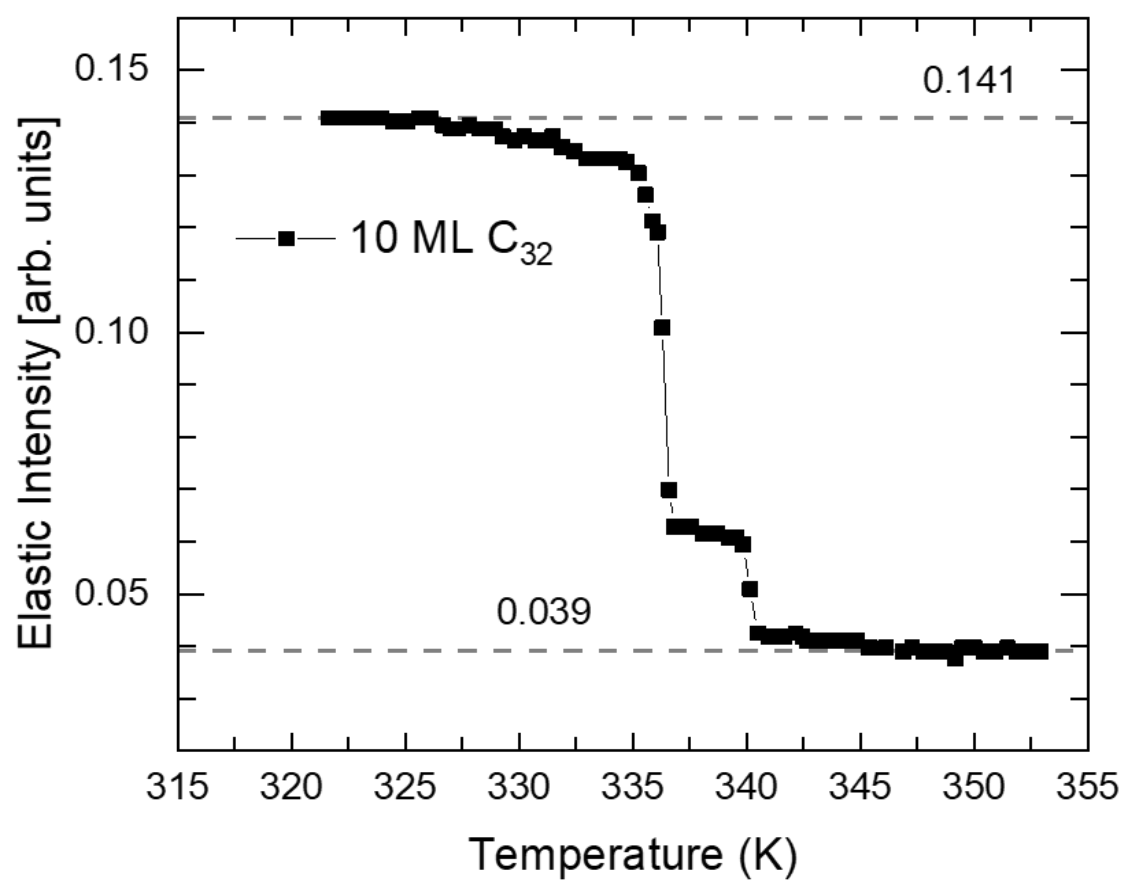

Figure B.1. Elastic scattering intensity summed over all 16 detectors vs. temperature as measured on the HFBS for a sample of 10 monolayers of $\mathrm{C}_{32}$ alkane material adsorbed onto $100 \mathrm{Si}$ wafers ( $5 \mathrm{~cm}$ dia., $300 \mu \mathrm{m}$ thick). Horizontal dashed lines are drawn at the measured intensities at the temperature extrema ( $353 \mathrm{~K}$ and $322 \mathrm{~K}$ ). Adapted from Ref. [167]. 
At a sample temperature above the bulk melting point $\left(T_{m}\right)$ of monoclinic $\mathrm{C}_{32}$ $(341 \mathrm{~K})$, the alkane molecules are in a fluid phase and the $\mathrm{H}$-atoms are considered mobile, i.e., they do not contribute to the elastic intensity. So, the elastic scattering is from the Si substrate plus some background. As shown in Fig. B.1, the monoclinic-totriclinic phase transition occurs at $\sim 337 \mathrm{~K}$ on cooling below $T_{m}$, after which the intensity levels-off indicating all the $\mathrm{H}$-atoms are fixed on a crystal lattice, i.e., they are considered immobile. By knowing the area per $\mathrm{C}_{32}$ and the total surface area of the silicon substrate (both sides of the 100 wafers), the calculation of number of $\mathrm{H}$-atoms $N_{H}$ is straightforward. The temperature dependence of the elastic intensity $I(T)$ can therefore be summarized as follows,

$$
I(T) \propto\left\{\begin{array}{cc}
\sigma(\mathrm{Si})+B G & T>341 K \\
\sigma\left(\mathrm{C}_{32}\right)+\sigma(\mathrm{Si})+B G & T<337 K
\end{array}\right.
$$

where $B G$ is an environmental (instrument) background intensity. Assuming the BG is temperature-independent, the difference in the intensity between temperature extrema is that which is contributed by the immobilization of $\mathrm{H}$-atoms in the alkane molecules, $I(\Delta T)=N_{H}\left(\mathrm{C}_{32}\right) \sigma\left(\mathrm{C}_{32}\right)$. We are now positioned to define the empirical scale factor $\alpha$, which is the fractional increase in elastic intensity between $353 \mathrm{~K}$ and $322 \mathrm{~K}$ :

$$
\begin{gathered}
\alpha \equiv \frac{I\left(T_{\min }\right)-I\left(T_{\max }\right)}{I\left(T_{\max }\right)}=\frac{I(322 K)-I(353 K)}{I(353 K)} \\
\alpha \cong 2.62
\end{gathered}
$$

This ratio $\alpha$ corresponds to the immobilization of approximately $1.43 \times 10^{21}$ hydrogen nuclei, assuming uniform coverage and phase purity of the $\mathrm{C}_{32}$ molecules. 


\section{B.2 Water-Film Thickness on $\mathrm{Cu}$ and $\mathrm{CuO}$ Surfaces}

We start with some preliminary calculations. Our sample consists of a stack of 100 copper foil disks, each measuring $5 \mathrm{~cm}$ in diameter and $12.7 \mu \mathrm{m}$ thick. The surface area of the 200 circular faces of these 100 disks is about $4,053 \mathrm{~cm}^{2}$. Using the bulk density of copper $\left(8.96 \mathrm{~g} / \mathrm{cm}^{3}\right)$, the mass of this sample is $23.06 \mathrm{~g}$, which is equal to $\sim 0.3629$ moles of copper or $\sim 2.185 \times 10^{23}$ copper atoms. The total incoherent scattering cross section $\Sigma(\mathrm{Cu})$ of the copper in this sample is obtained by scaling the incoherent scattering cross section of a single copper atom ( 0.55 barn) by the number of copper atoms $N_{C u}$ in the 100 foils,

$$
\begin{gathered}
\Sigma(\mathrm{Cu})=N_{\mathrm{Cu}} * \sigma_{\text {inc }}(\mathrm{Cu}) \\
=2.185 \times 10^{23} * 0.55 \text { barn } \\
\Sigma(\mathrm{Cu}) \cong 1.202 \times 10^{23} \text { barn }
\end{gathered}
$$

In ordered to calibrate our measurements against the alkane standard, we need to account for the difference in the incoherent scattering from the different substrates, Recall there are about $3.04 \times 10^{24} \mathrm{Si}$ atoms in the Si substrates, so we define the empirical scale factor $\gamma$, which is the ratio of the total incoherent scattering of copper to silicon,

$$
\begin{gathered}
\Sigma(\mathrm{Si})=3.04 \times 10^{24} * 0.004 \text { barn }=1.22 \times 10^{22} \text { barn } \\
\gamma \equiv \frac{\Sigma(\mathrm{Cu})}{\Sigma(\mathrm{Si})} \cong 9.896
\end{gathered}
$$


Figure B.2 shows the temperature-dependent elastic scans collected on cooling and heating at $0.08 \mathrm{~K} / \mathrm{min}$ in the temperature range of $\sim 200-280 \mathrm{~K}$ for our three samples: $\mathrm{CuO}$ with (a) $60 \mu \mathrm{l}$ of added $\mathrm{H}_{2} \mathrm{O}$, (b) $10 \mu \mathrm{l}$ of $\mathrm{H}_{2} \mathrm{O}$; and, (c) bare-Cu foils with $60 \mu \mathrm{l}$ of $\mathrm{H}_{2} \mathrm{O}$. The elastic intensity was summed over all 16 detectors, which span a wave vector transfer $(Q)$ range of $0.25-1.75 \AA^{-1}$, and then normalized to the incident beam monitor located just upstream of the sample position. The elastic intensities at the highest and lowest temperatures in the cooling scans of each sample are listed in Table B.2.

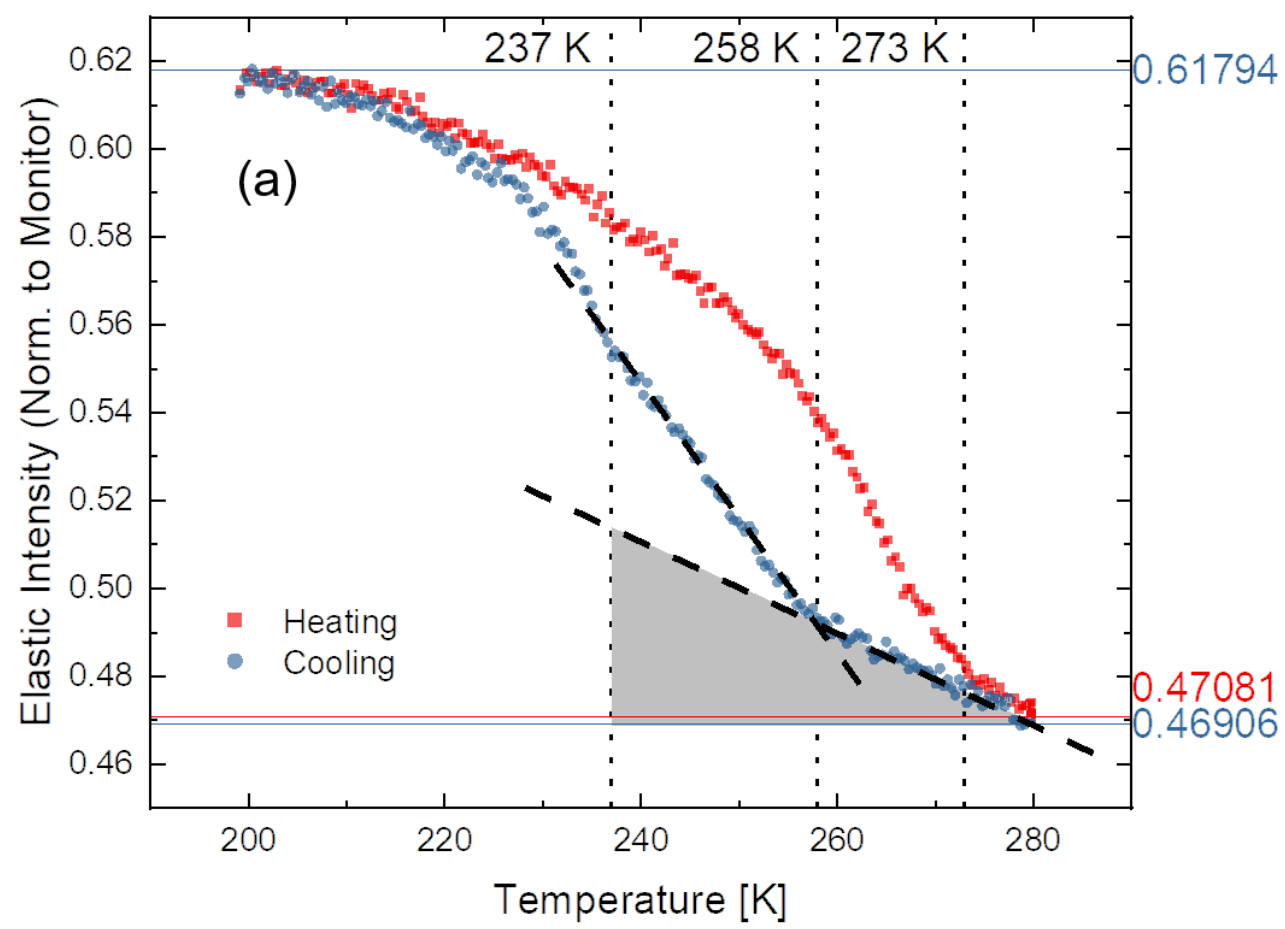



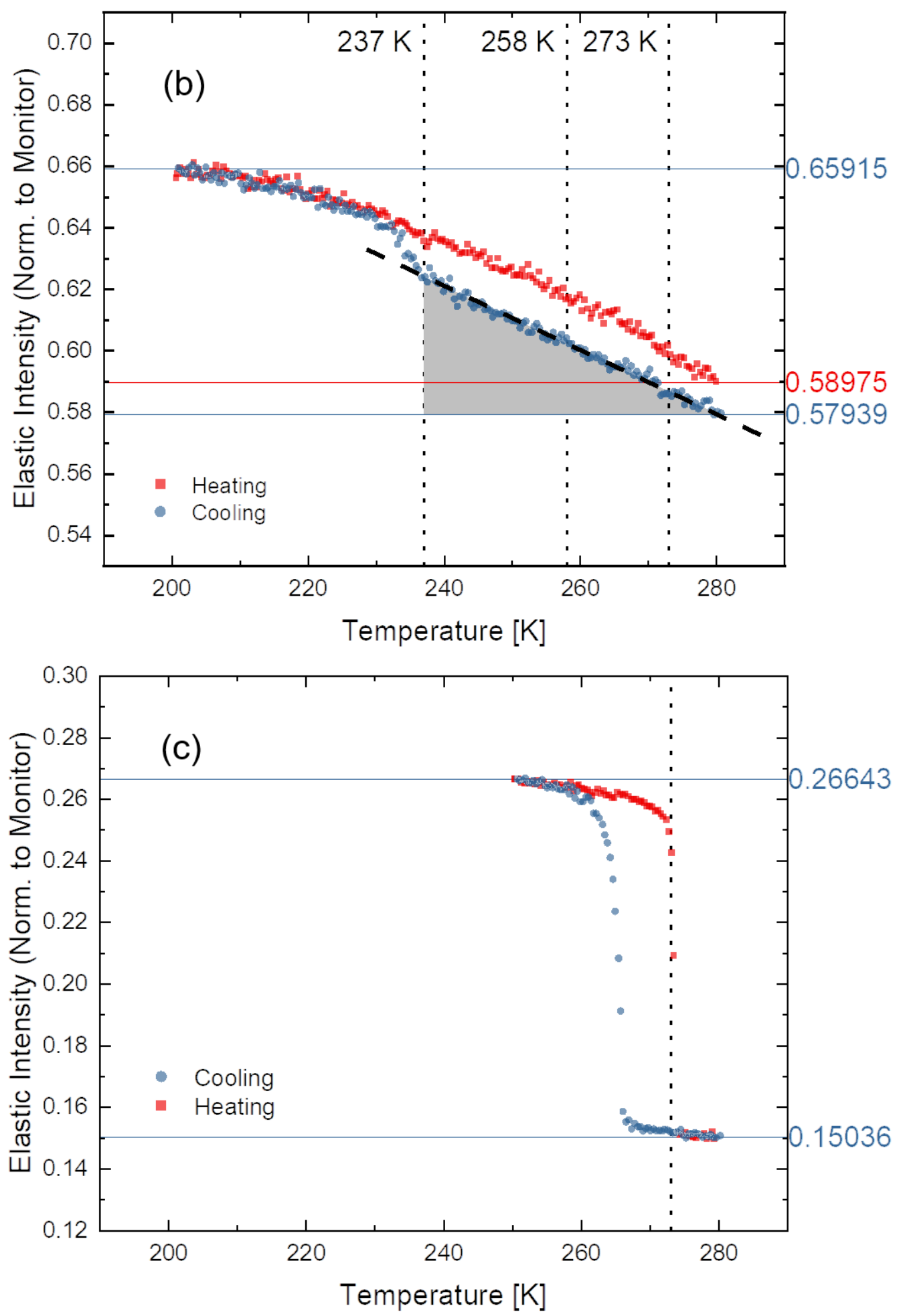

Figure B.2. Intensity of elastically scattered neutrons vs. temperature, summed over all 16 detectors (all $Q$ ) and normalized to the incident beam monitor. Data are shown for three samples: $\mathrm{CuO}$ with (a) $60 \mu \mathrm{l}$ of added $\mathrm{H}_{2} \mathrm{O}$, (b) $10 \mu \mathrm{l}$ of $\mathrm{H}_{2} \mathrm{O}$; and, (c) bare-Cu foils with $60 \mu \mathrm{l}$ of $\mathrm{H}_{2} \mathrm{O}$. Horizontal lines are drawn at the intensities at the highest ( $\left.280 \mathrm{~K}\right)$ and lowest temperatures ( $200 \mathrm{~K}$ or $250 \mathrm{~K})$ for each sample and summarized in Table B.2. 


\begin{tabular}{|c|c|c|c|}
\hline & $\mathrm{CuO}+60 \mu \mathrm{l} \mathrm{H}_{2} \mathrm{O}$ & $\mathrm{CuO}+10 \mu \mathrm{l} \mathrm{H}_{2} \mathrm{O}$ & $\mathrm{Cu}+60 \mu \mathrm{l} \mathrm{H} \mathrm{H}_{2} \mathrm{O}$ \\
\hline$T_{\min }[\mathrm{K}]$ & 200.5 & 201.04 & 250.81 \\
\hline$I\left(T_{\min }\right)$ & 0.61794 & 0.65915 & 0.26643 \\
\hline$T_{\max }[\mathrm{K}]$ & 279.92 & 280.85 & 280.38 \\
\hline$I\left(T_{\max }\right)$ & 0.46906 & 0.57939 & 0.15036 \\
\hline$\Delta I$ & 0.14888 & 0.07976 & 0.11607 \\
\hline$\beta$ & 0.31740 & 0.13766 & 0.77194 \\
\hline$h[\AA]$ & 1,952 & 1,048 & 1,520 \\
\hline
\end{tabular}

Table B.2. Monitor-normalized elastic intensity $(I(T))$ measured at the minimum $\left(T_{\min }\right)$ and maximum $\left(T_{\max }\right)$ temperatures of the elastic scans shown in Fig. B.2. Their difference $(\Delta I)$ is used to calculate the empirical unitless scale factor $\beta$.

Our analysis begins with the sample of untreated copper foils where we assume the elastic intensity $I(T)$ above the melting point of water $T_{m}\left(273 \mathrm{~K}\right.$ for bulk $\left.\mathrm{H}_{2} \mathrm{O}\right)$ is dominated by the scattering from the copper substrates plus some background. Likewise, the elastic intensity below $T_{m}$ is a sum of the scattering from copper, background, and the $\mathrm{H}$-atoms in water molecules that are immobile or "frozen" on the experimental time scale $(\sim 4 \mathrm{~ns})$,

$$
I(T) \propto\left\{\begin{array}{cl}
\sigma(\mathrm{Cu})+B G & T>T_{m} \\
\sigma\left(\mathrm{H}_{2} \mathrm{O}\right)+\sigma(\mathrm{Cu})+B G & T \leq T_{m}
\end{array}\right.
$$

From the elastic intensities determined graphically in Fig. B.2c, we define the empirical parameter $\beta$, which is the fractional increase in elastic intensity between the highest and lowest temperatures (identical to the definition of $\alpha$ ),

$$
\beta \equiv \frac{I\left(T_{\min }\right)-I\left(T_{\max }\right)}{I\left(T_{\max }\right)}=\frac{A \Sigma\left(\mathrm{H}_{2} \mathrm{O}\right)}{A \Sigma(\mathrm{Cu})+B G}
$$

Here, the coefficient $A$ is a proportionality constant which is sensitive to data reduction and normalization methods. Moreover, the quantity $A$ is physically a factor which 
converts neutron count to intensity. The ratio $\beta / \alpha$ tells us how many times bigger the fractional increase in intensity due to water freezing on the $100 \mathrm{Cu}$ foils compares to the $1.43 \times 10^{21} \mathrm{H}$-atoms immobilized in the $\mathrm{C}_{32}$ molecules that are adsorbed on $100 \mathrm{Si}$ wafers. In order to convert $\beta$ to its corresponding value for water freezing on $\mathrm{Si}$, we need to multiply by $\gamma$. That is, the number of $\mathrm{H}$-atoms in the water freezing on a $\mathrm{Cu}$ substrate would be,

$$
\frac{\gamma \beta}{\alpha} * 1.43 \times 10^{21} \mathrm{H} \text { atoms } \cong 4.11 \times 10^{21} \mathrm{H} \text { atoms }
$$

So, there are $4.11 \times 10^{21} \mathrm{H}$ atoms or $2.05 \times 10^{21}$ water molecules freezing onto the 100 untreated $\mathrm{Cu}$ foils. This number also corresponds to $3.41 \times 10^{-3}$ moles, $6.14 \times 10^{-2}$ grams, or $6.14 \times 10^{-2} \mathrm{~cm}^{3}$ of frozen water. Uniformly distributing the volume of water $\left(6.14 \times 10^{-2} \mathrm{~cm}^{3}\right)$ over the total surface area of the $100 \mathrm{Cu}$ foils $\left(4.05 \times 10^{3} \mathrm{~cm}^{2}\right)$ equates to an equivalent slab thickness $h_{C u+60 \mu l}$ of roughly $1,520 \AA$.

Since the $\mathrm{CuO}$ samples were measured under the same experimental conditions on the HFBS, to calculate their effective water slab thickness we do not consider the fractional in the elastic intensity between $\sim 280 \mathrm{~K}$ and $\sim 200 \mathrm{~K}$. Rather, we use the increase in the elastic intensity scaled to the incident monitor, $\Delta I=I(200 \mathrm{~K})-$ $I(280 K)$. That is,

$$
\begin{aligned}
& h_{C u O+60 \mu l}=h_{C u+60 \mu l}\left(\frac{\Delta I_{C u O+60 \mu l}}{\Delta I_{C u+60 \mu l}}\right)=1,952 \AA \\
& h_{C u O+10 \mu l}=h_{C u+60 \mu l}\left(\frac{\Delta I_{C u O+10 \mu l}}{\Delta I_{C u+60 \mu l}}\right)=1,048 \AA
\end{aligned}
$$


The above quantities are given in Table B.2 as well. The above slab thicknesses are applicable for perfectly flat and chemically homogeneous solid surfaces on which the wetting is considered ideal. 


\section{Appendix C - Neutron Diffraction Bragg}

\section{Peak Analysis at the MU Research Reactor}

This Appendix supplements the analysis of diffraction patterns presented in Sec 3.4. All diffraction scans were recorded using the powder diffractometer at D-port at MURR, assuming a neutron wavelength of $1.485 \AA$. The detector system consists of a vertical stack of five linear position-sensitive detectors (PSDs) mounted on a movable arm that covers scattering angles in the range $0^{\circ}<2 \theta<105^{\circ}$. In a single arm position, the detectors subtend a scattering arc of $2 \theta \sim 20^{\circ}$. In most cases, we set the detectors to count neutrons in the scattering range $19.45^{\circ}<2 \theta<39.40^{\circ}$ where the most intense Bragg peaks corresponding to hexagonal and cubic ice can be found [136].

\section{C.1 Temperature Dependence of the Diffraction}

\section{Patterns}

The neutron diffraction patterns for CuO-coated copper foils hydrated with $120 \mu \mathrm{l}$ of $\mathrm{D}_{2} \mathrm{O}$ are shown in Fig. C.1. By inspection, the patterns do not contain Bragg peaks that could be indexed to crystalline ice. It was after adding a total of $240 \mu \mathrm{l}$ of $\mathrm{D}_{2} \mathrm{O}$ to the sample cell that we observed three Bragg peaks develop on cooling from 265 K to 200 $\mathrm{K}$ that could be indexed to hexagonal ice. 


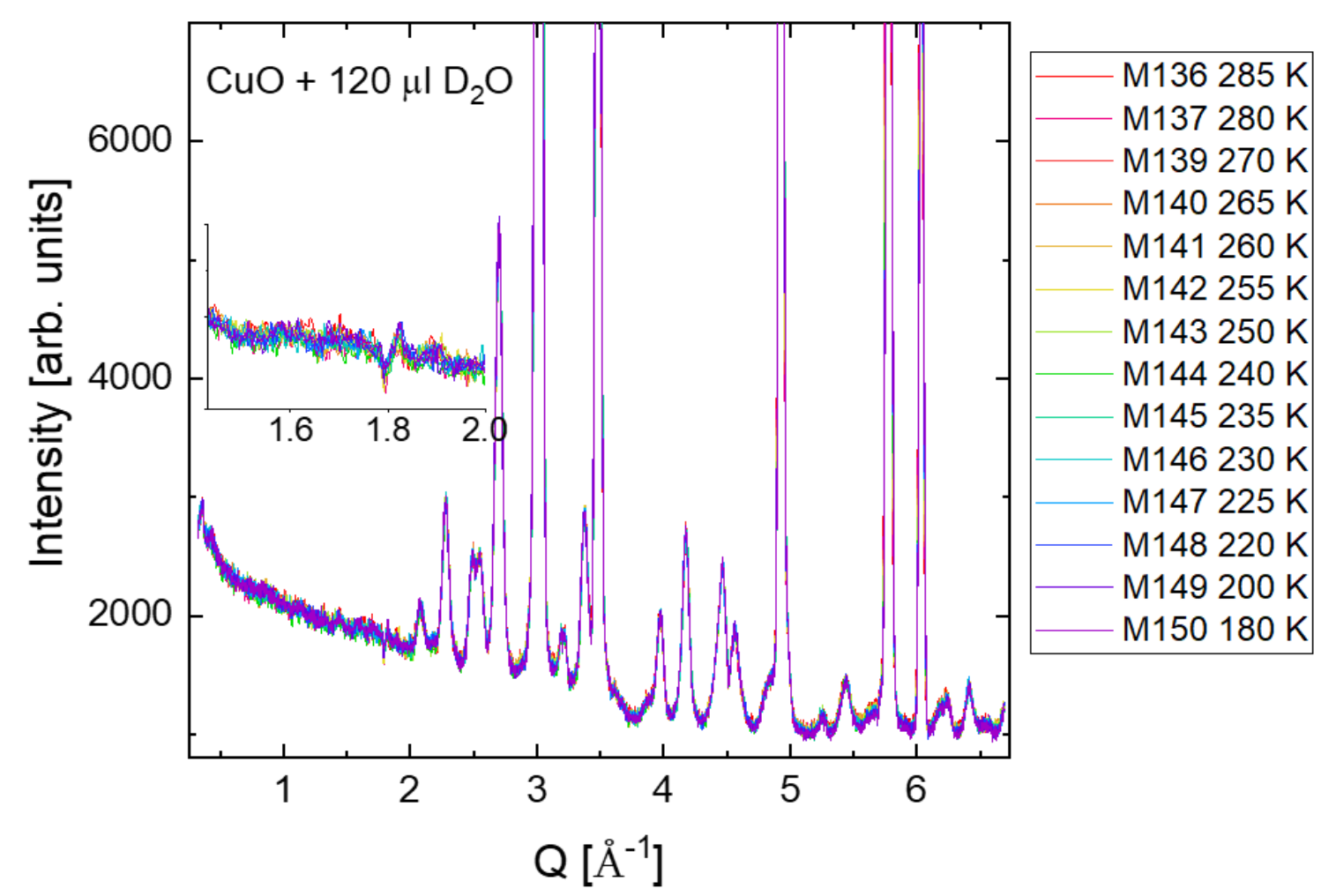

Figure C.1. Diffraction patterns of the CuO-coated sample hydrated with $120 \mu 1$ of $\mathrm{D}_{2} \mathrm{O}$ measured on cooling to $180 \mathrm{~K}$. Inset shows the low- $Q$ region where the most intense peaks of hexagonal and cubic ice would be located [136]. M136-M150 refer to scan numbers on the PSD.

Several diffractions patterns for the $\mathrm{CuO}$ sample hydrated with $240 \mu 1 \mathrm{D}_{2} \mathrm{O}$ are shown in Fig. C.2 for several temperatures on cooling from $295 \mathrm{~K}$ to $200 \mathrm{~K}$. Vertical lines are drawn at the calculated peak positions for the (100), (002), and (101) reflections of hexagonal $\mathrm{D}_{2} \mathrm{O}$ ice at $265 \mathrm{~K}[\mathbf{1 3 6}]$. The scatter in the data makes fitting the Bragg peaks to a Gaussian or Lorentzian function difficult. However, in most cases, the most intense (100) peak could be fit with a Gaussian function of the form,

$$
y=y_{0}+\frac{A}{\left(w \sqrt{\frac{\pi}{2}}\right)} \exp \left[-2\left(\frac{x-x_{c}}{w}\right)^{2}\right]
$$


where $y_{0}$ is free parameter and $x_{c}, w$, and $A$ are the peak center, width, and integrated intensity, respectively. A representative fit to the data collected at $\sim 200 \mathrm{~K}$ is shown in Fig. C.3. By comparing the observed and calculated positions of the $\mathrm{D}_{2} \mathrm{O}(100)$ peak, one notices that the observed peak is shifted to lower scattering angles (lower $Q$ ) than calculated. It turns out this observed peak shift is an instrument effect and details are provided in the next section.

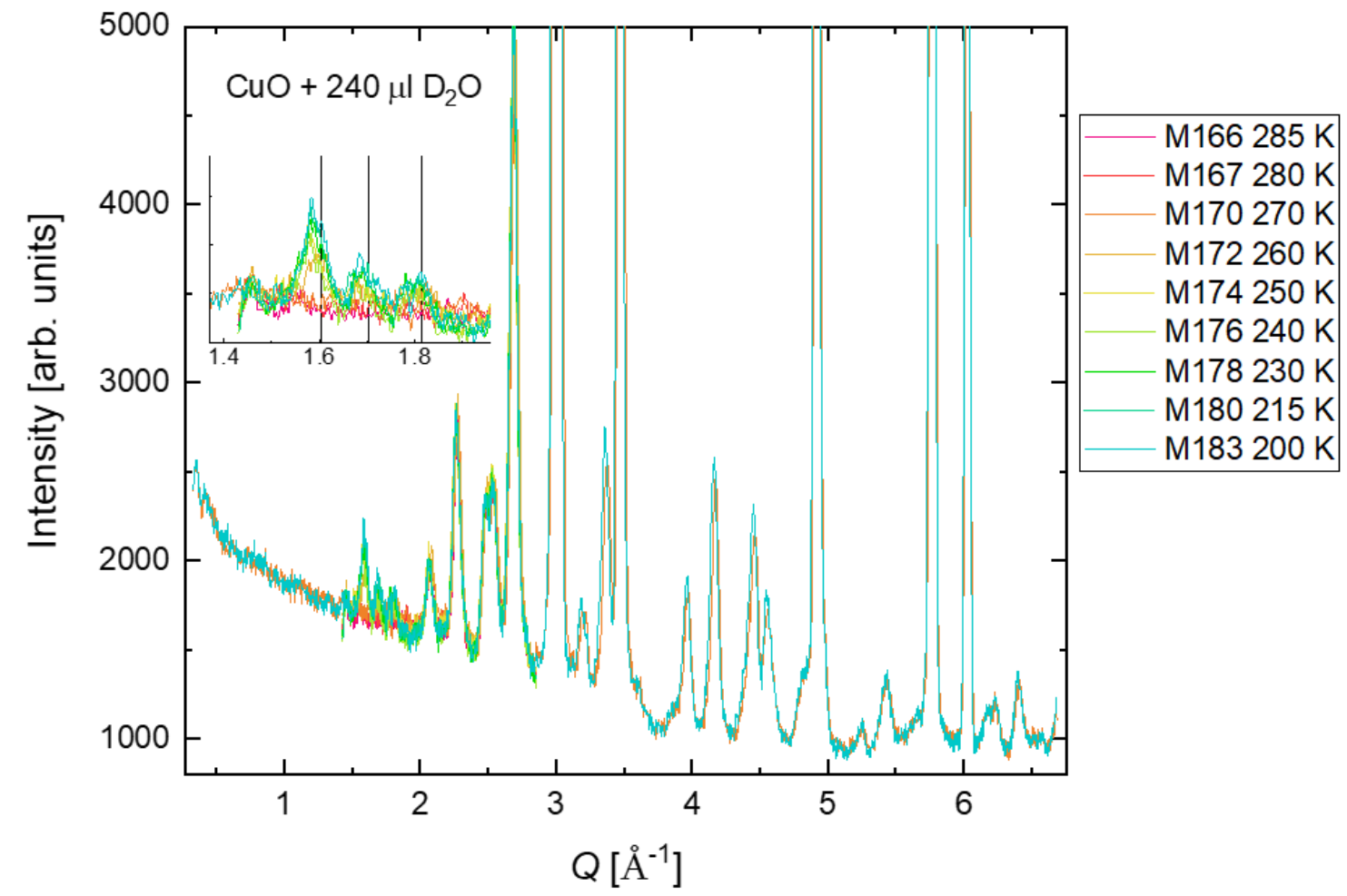

Figure C.2. Diffraction scans of the CuO-coated sample hydrated with $240 \mu \mathrm{l}$ of $\mathrm{D}_{2} \mathrm{O}$ measured on cooling to $200 \mathrm{~K}$. Inset shows the three Bragg peaks indexed to hexagonal ice. M166-M183 refer to scan numbers on the PSD.

As discussed in Sec. 3.4, the background intensity of the CuO-coated samples was greater than that of the bare-Cu samples. Furthermore, as shown in Fig. C.4, the magnitude of the background level was independent of hydration level. 


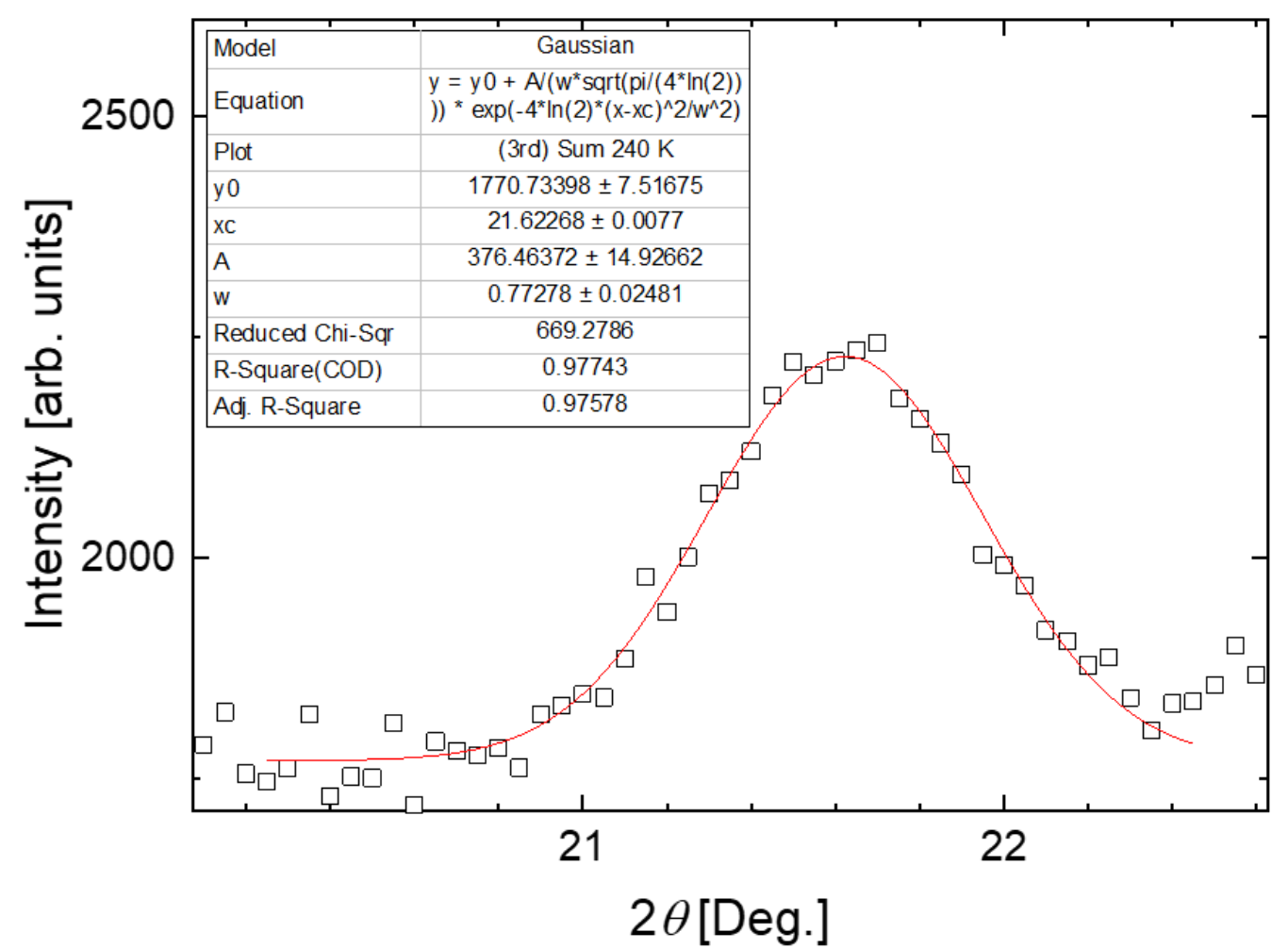

Figure C.3. Example of a Gaussian line shape fit (Eq. (C.1)) to the (100) Bragg peak of $\mathrm{D}_{2} \mathrm{O}$ hexagonal ice [136]. Inset shows the results and goodness of the fit.

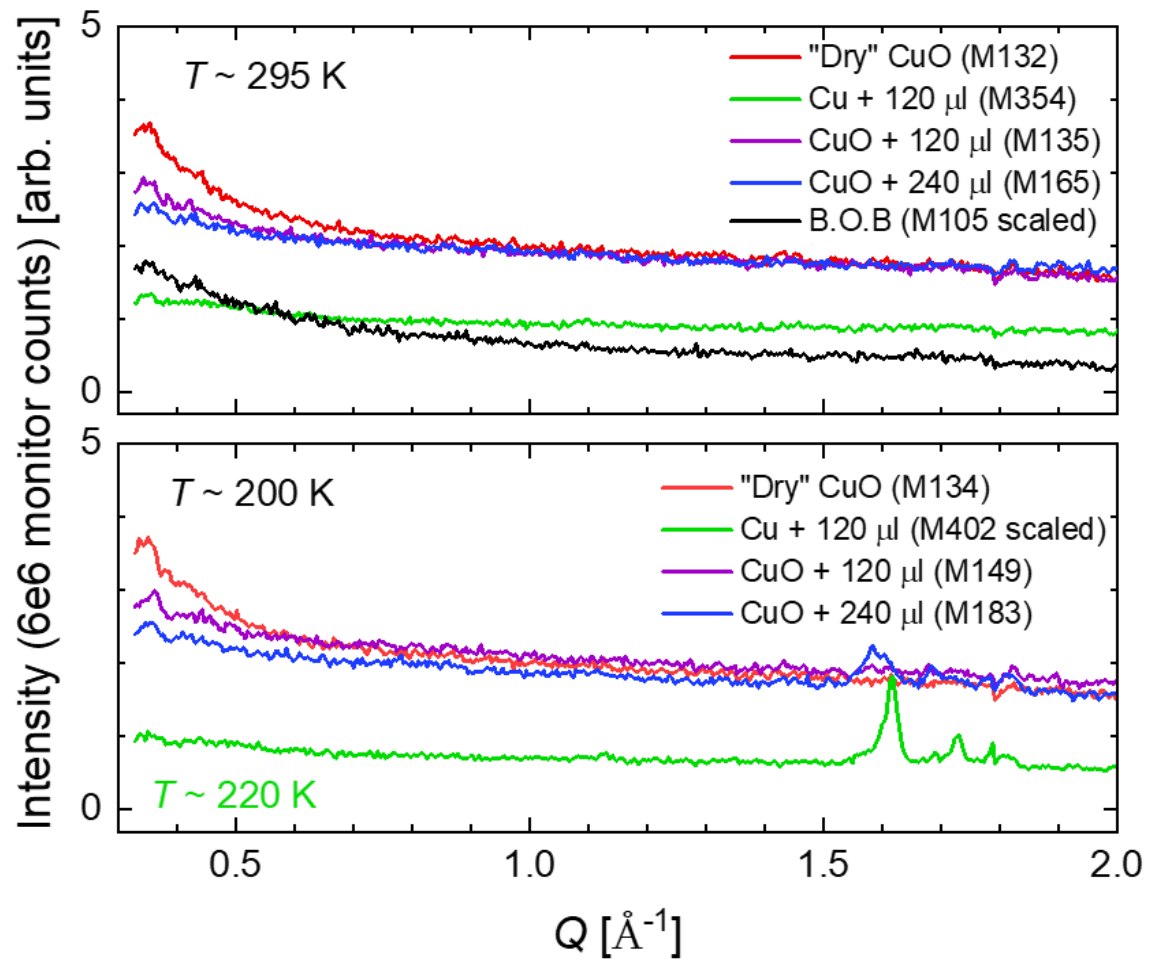

Figure C.4. Background levels of several diffraction scans and beam-open background (B.O.B.) 


\section{C.2 Correction to Bragg Peak Position}

In the second cooling cycle of the high hydration $\left(240 \mu 1 \mathrm{D}_{2} \mathrm{O}\right) \mathrm{CuO}$ sample, an anomalous shift in the Bragg peak positions of copper, $\mathrm{CuO}$, and $\mathrm{D}_{2} \mathrm{O}$ was observed around $260 \mathrm{~K}$. This angular shift is illustrated in Fig. C.5 where the position of the fiducial $\mathrm{Cu}(111)$ peak (in degrees) is plotted versus temperature. With some investigation we found the offset's origin was mechanical, which was addressed and promptly fixed.

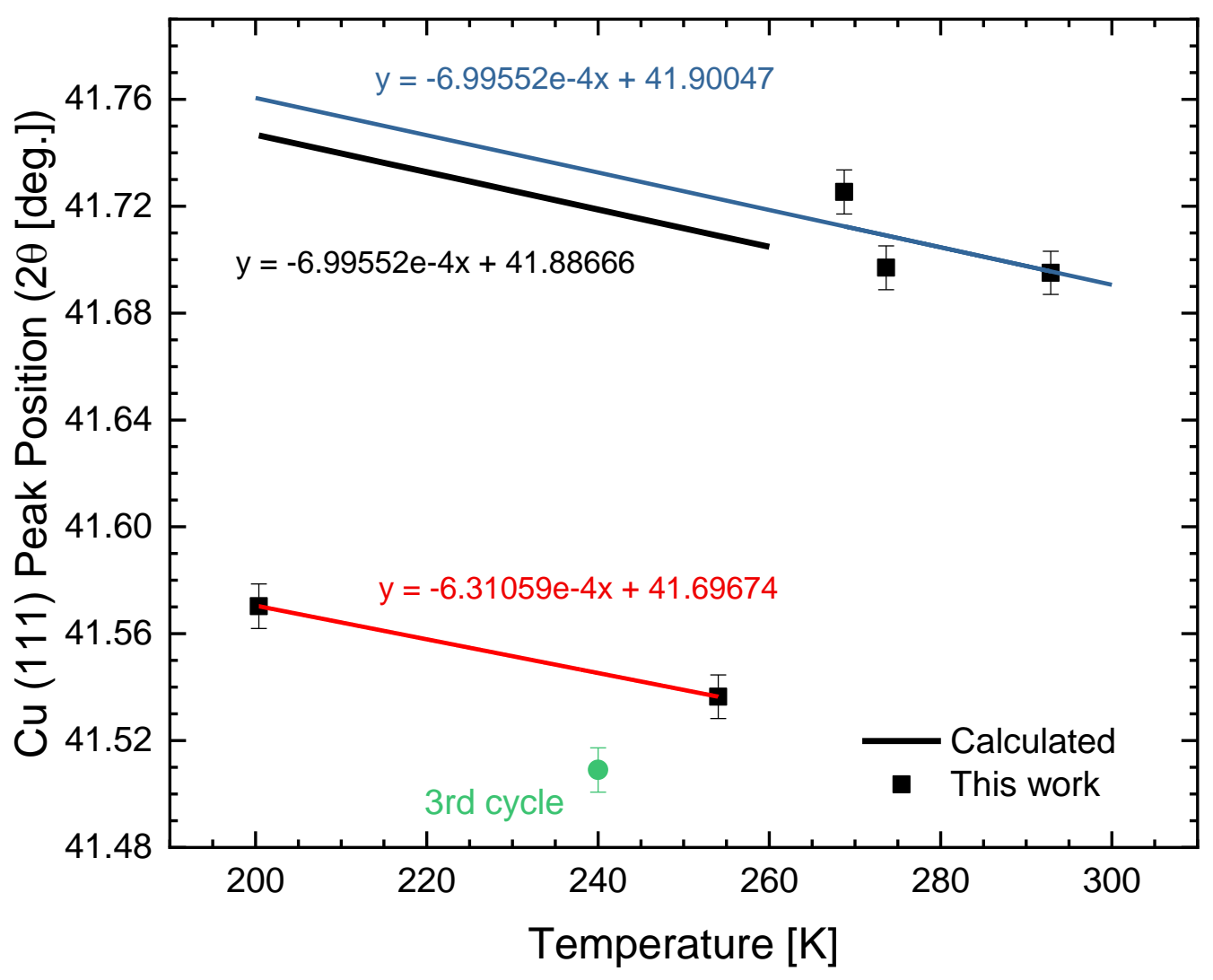

Figure C.5. $\mathrm{Cu}(111)$ Bragg peak position vs. temperature that illustrates an instrumental shift around $260 \mathrm{~K}$. The black line consists of calculated positions of the $\mathrm{Cu}(111)$ peak extrapolated from room temperature to $200 \mathrm{~K}$ (see text). The red line is a linear fit to the two low-temperature data points and the blue line is a fit to the high-temperature data where its slope has been fixed to that of the black line, following the bulk lattice contraction. 
The following procedure was used to correct for the angular offset of the $\mathrm{D}_{2} \mathrm{O}$ peaks. First, the $\mathrm{Cu}(111) d$-spacing was calculated using the lattice parameter at $293 \mathrm{~K}\left(a_{0}=\right.$ $3.6148 \AA$ ). Then, using the linear thermal expansion coefficient of copper $(\alpha=1.604 \mathrm{E}-5$ $\left.\mathrm{K}^{-1}, \sigma \sim 4.8 \mathrm{E}-7[\mathbf{2 0 5}]\right)$, the copper lattice parameter was recalculated for temperatures in the range 200-300 K:

$$
\Delta a=a_{0} * \alpha \Delta T
$$

The black line in Fig. C.5 shows the results of the re-calculated $\mathrm{Cu}(111)$ peak position. Next, the data above (blue) and below (red) the angular shift at $\sim 260 \mathrm{~K}$ were fit to lines as shown in Fig. C.5 where the slope of the blue line was fixed to that of bulk copper. The difference between the red and blue lines is therefore equal to the correction term $\left(2 \theta_{0}\right)$ added to the data below the discontinuity $(<260 \mathrm{~K})$. Finally, the peak position of $\mathrm{D}_{2} \mathrm{O}(100)$ was determined for temperatures between $200 \mathrm{~K}$ and $300 \mathrm{~K}$ (see right-most column in Table C.1); The observed and corrected $\mathrm{D}_{2} \mathrm{O}(100)$ positions are also illustrated in Fig. C.6.

\begin{tabular}{ccccccc}
$\begin{array}{c}\text { Temp. } \\
{[\mathrm{K}]}\end{array}$ & $\begin{array}{c}\mathbf{D}_{2} \mathbf{O}(\mathbf{1 0 0}) \\
{\left[{ }^{\circ} 2 \theta\right]}\end{array}$ & $\begin{array}{c}\text { Blue line } \\
(\text { Fig. C.5 })\end{array}$ & $\begin{array}{c}\text { Red line } \\
(\text { Fig. C.5) }\end{array}$ & $\begin{array}{c}\text { Blue }- \\
\text { Red }\left[{ }^{\circ} 2 \theta\right]\end{array}$ & $\begin{array}{c}\text { Corrected } \\
{\left[{ }^{\circ} 2 \theta\right]}\end{array}$ & $\begin{array}{c}\text { Corrected } \\
(Q[\AA-1])\end{array}$ \\
\hline 260.00 & 21.733 & 41.719 & 41.533 & 0.186 & 21.919 & 1.609 \\
254.03 & 21.610 & 41.723 & 41.536 & 0.186 & 21.796 & 1.600 \\
249.15 & 21.509 & 41.726 & 41.540 & 0.187 & 21.696 & 1.593 \\
244.25 & 21.521 & 41.730 & 41.543 & 0.187 & 21.708 & 1.593 \\
239.37 & 21.529 & 41.733 & 41.546 & 0.187 & 21.716 & 1.594 \\
234.50 & 21.549 & 41.736 & 41.549 & 0.188 & 21.737 & 1.596 \\
229.63 & 21.574 & 41.740 & 41.552 & 0.188 & 21.762 & 1.597 \\
224.77 & 21.580 & 41.743 & 41.555 & 0.188 & 21.769 & 1.598 \\
215.03 & 21.618 & 41.750 & 41.561 & 0.189 & 21.807 & 1.601 \\
210.19 & 21.596 & 41.753 & 41.564 & 0.189 & 21.786 & 1.599 \\
205.27 & 21.630 & 41.757 & 41.567 & 0.190 & 21.820 & 1.602 \\
200.39 & 21.654 & 41.760 & 41.570 & 0.190 & 21.844 & 1.603
\end{tabular}

Table C.1. $\mathrm{D}_{2} \mathrm{O}$ peak position corrected for instrument effects using $\mathrm{Cu}(111)$ fiducial. 


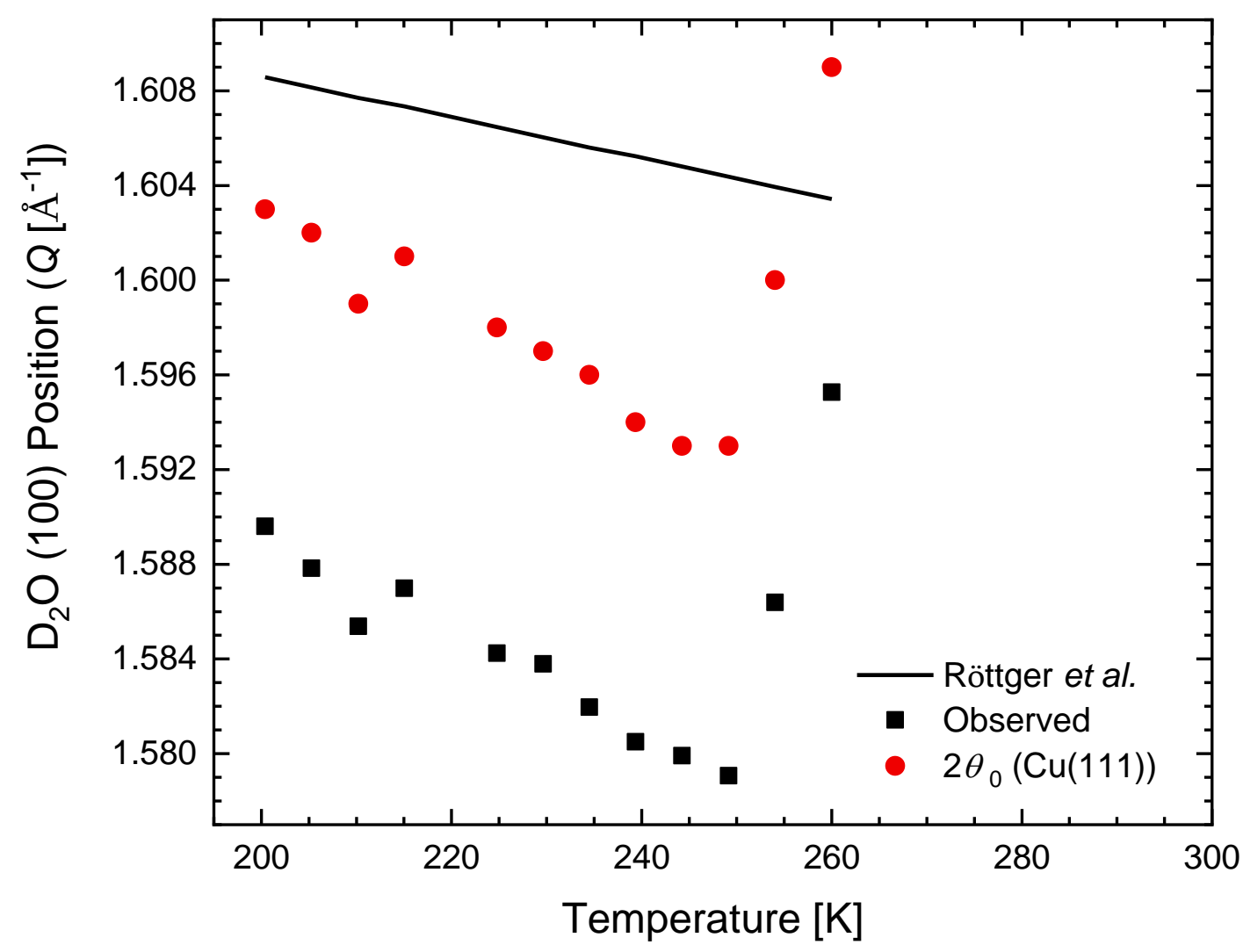

Figure C.6. Observed (black points) and corrected (red points) positions of the $\mathrm{D}_{2} \mathrm{O}(100)$ peak as a function of temperature. Also shown are the reference data for bulk $\mathrm{D}_{2} \mathrm{O}$ calculated from Ref. [136].

Because diffraction patterns were recorded within a narrow $Q$ range that excludes the $\mathrm{Cu}(111)$ peak, we performed an identical correction using the $\mathrm{CuO}(111)$ peak as a fiducial. As in Fig. C.5, the peak position of $\mathrm{CuO}(111)$ is plotted in Fig. C.7 vs. temperature where, again, a discontinuity is observed around $260 \mathrm{~K}$. Data above and below the discontinuity were fit with lines and compared with calculated peak positions using the thermal expansion coefficient of $\alpha=5.1 \mathrm{E}-6 \mathrm{~K}^{-1}$ [206]. Results are summarized in Fig. C.8 and Table C.2 below. Clearly, the $\mathrm{CuO}(111)$ correction provides a better agreement between observed and calculated positions of the $\mathrm{D}_{2} \mathrm{O}(100)$ peak compared to the $\mathrm{Cu}(111)$ correction. The data presented in Sec. 3.4 are therefore corrected using the $\mathrm{CuO}(111)$ fiducial. 


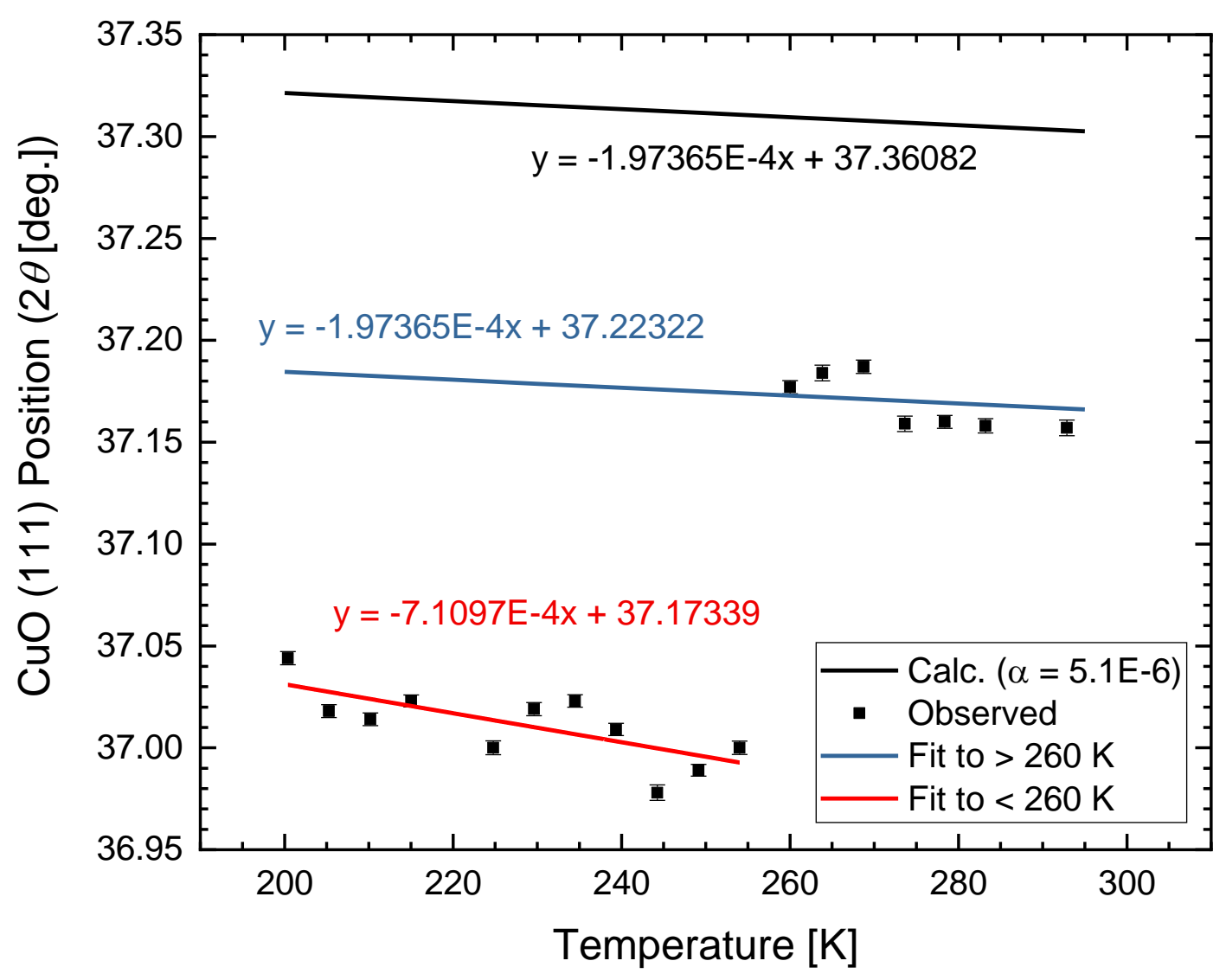

Figure C.7. $\mathrm{CuO}(111)$ Bragg peak position vs. temperature that illustrates a shift around $260 \mathrm{~K}$. The black line consists of calculated positions of the $\mathrm{CuO}(111)$ peak extrapolated from room temperature to $200 \mathrm{~K}$ (see text). The red line is a linear fit to the lowtemperature data points and the blue line is a fit to the high-temperature data where its slope has been fixed to that of the black line, following the bulk lattice contraction. 


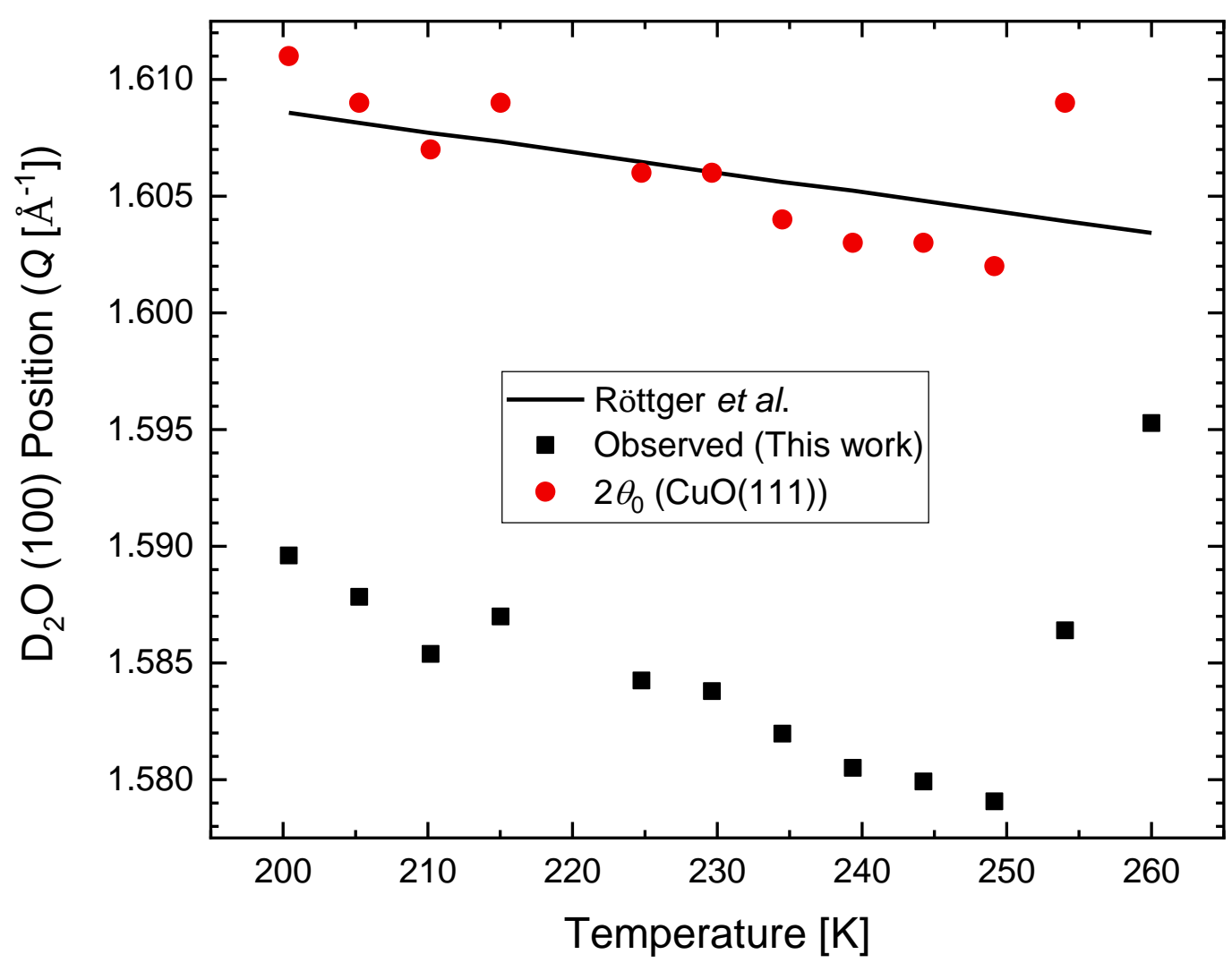

Figure C.8. Observed (black points) and corrected (red points) positions of the $\mathrm{D}_{2} \mathrm{O}(100)$ peak vs. temperature. Also shown are the reference data for bulk $\mathrm{D}_{2} \mathrm{O}$ [136].

\begin{tabular}{ccccccc}
$\begin{array}{c}\text { Temp. } \\
{[\mathrm{K}]}\end{array}$ & $\begin{array}{c}\mathbf{D}_{2} \mathbf{O}(\mathbf{1 0 0}) \\
{\left[{ }^{\circ} 2 \theta\right]}\end{array}$ & $\begin{array}{c}\text { Blue line } \\
(\text { Fig. C.7) }\end{array}$ & $\begin{array}{c}\text { Red line } \\
(\text { Fig. C.7) }\end{array}$ & $\begin{array}{c}\text { Blue - Red } \\
{\left[{ }^{\circ} 2 \theta\right]}\end{array}$ & $\begin{array}{c}\text { Corrected } \\
{\left[{ }^{\circ} 2 \theta\right]}\end{array}$ & $\begin{array}{c}\text { Corrected } \\
\left(Q\left[\AA^{-1}\right]\right)\end{array}$ \\
\hline 254.03 & 21.610 & 37.311 & 36.993 & 0.318 & 21.928 & 1.609 \\
249.15 & 21.509 & 37.312 & 36.996 & 0.315 & 21.825 & 1.602 \\
244.25 & 21.521 & 37.313 & 37.000 & 0.313 & 21.834 & 1.603 \\
239.37 & 21.529 & 37.314 & 37.003 & 0.310 & 21.839 & 1.603 \\
234.50 & 21.549 & 37.315 & 37.007 & 0.308 & 21.857 & 1.604 \\
229.63 & 21.574 & 37.315 & 37.010 & 0.305 & 21.880 & 1.606 \\
224.77 & 21.580 & 37.316 & 37.014 & 0.303 & 21.883 & 1.606 \\
215.03 & 21.618 & 37.318 & 37.021 & 0.298 & 21.916 & 1.609 \\
210.19 & 21.596 & 37.319 & 37.024 & 0.295 & 21.892 & 1.607 \\
205.27 & 21.630 & 37.320 & 37.027 & 0.293 & 21.923 & 1.609 \\
200.39 & 21.654 & 37.031 & 37.031 & 0.290 & 21.945 & 1.611
\end{tabular}

Table C.2. $\mathrm{D}_{2} \mathrm{O}$ peak position corrected for instrument effects using $\mathrm{CuO}(111)$ fiducial. 


\section{C.3 $\mathrm{D}_{2} \mathrm{O}$ Ice Particle Domain Size Calculation}

The following expression was used to estimate the average particle/domain size $L$ of the $\mathrm{D}_{2} \mathrm{O}$ ice,

$$
L=\frac{K \lambda}{B_{h k l} \cos \frac{2 \theta}{2}}
$$

Here, $\lambda$ is the neutron wavelength $(1.485 \AA), 2 \theta$ is the scattering angle, $K$ is the Scherrer constant $^{1}(0.9)$, and $B_{h k l}$ is the resolution-broadened full-width at half-maximum (FWHM) of the $\mathrm{D}_{2} \mathrm{O}(100)$ Bragg peak:

$$
B_{h k l}=\sqrt{B_{o b s}^{2}-B_{r e s}^{2}}
$$

where $B_{\text {obs }}$ is the observed FWHM of the $\mathrm{D}_{2} \mathrm{O}(100)$ peak and $B_{\text {res }}$ is the FWHM of the silicon powder standard (111) peak $(\sim 0.00469)$, both in radians. The procedure that was used to calculate the $\mathrm{D}_{2} \mathrm{O}$ domain size is as follows.

1. Extract the FWHM of the $\mathrm{D}_{2} \mathrm{O}(100)$ peak from a Gaussian fit

2. Convert the FWHM to the appropriate units

3. Calculate $B_{h k l}$ as shown above in Eq. (C.4)

4. Calculate $L$ as shown above in Eq. (C.3)

The data used to calculate the domain size are provided below in Table C.3. The average domain size $L$ is $307.22 \AA$ with a standard deviation of $57.93 \AA$.

\footnotetext{
${ }^{1}$ A more detailed analysis of the domain size would include the following to estimate the Scherrer constant: how the peak width is defined, shape of the crystal, particle size distribution, and background subtraction. In this case, the value of 0.9 is reasonable as it commonly applies to many types of materials.
} 


\begin{tabular}{cccccc}
$\begin{array}{c}\mathrm{D}_{2} \mathrm{O}(100) \\
\text { width } \\
(2 \theta \text { deg })\end{array}$ & error & $\begin{array}{c}\mathrm{D}_{2} \mathrm{O}(100) \\
\text { width } \\
(\theta \text { deg })\end{array}$ & $\begin{array}{c}\mathrm{D}_{2} \mathrm{O}(100) \\
\text { width (rad. })\end{array}$ & $\begin{array}{c}B_{h k l} \\
(\text { rad. })\end{array}$ & $\begin{array}{c}L \\
(\AA)\end{array}$ \\
\hline $8.205 \mathrm{E}-01$ & $2.904 \mathrm{E}-02$ & 0.410 & $7.160 \mathrm{E}-03$ & $5.410 \mathrm{E}-03$ & 251.56 \\
$7.532 \mathrm{E}-01$ & $5.647 \mathrm{E}-02$ & 0.377 & $6.573 \mathrm{E}-03$ & $4.605 \mathrm{E}-03$ & 295.44 \\
$6.307 \mathrm{E}-01$ & $4.520 \mathrm{E}-02$ & 0.315 & $5.504 \mathrm{E}-03$ & $2.881 \mathrm{E}-03$ & 472.26 \\
$7.348 \mathrm{E}-01$ & $5.448 \mathrm{E}-02$ & 0.367 & $6.413 \mathrm{E}-03$ & $4.373 \mathrm{E}-03$ & 311.07 \\
$7.223 \mathrm{E}-01$ & $7.401 \mathrm{E}-02$ & 0.361 & $6.303 \mathrm{E}-03$ & $4.212 \mathrm{E}-03$ & 323.03 \\
$7.259 \mathrm{E}-01$ & $4.399 \mathrm{E}-02$ & 0.363 & $6.334 \mathrm{E}-03$ & $4.258 \mathrm{E}-03$ & 319.54 \\
$9.024 \mathrm{E}-01$ & $5.605 \mathrm{E}-02$ & 0.451 & $7.874 \mathrm{E}-03$ & $6.325 \mathrm{E}-03$ & 215.09 \\
$7.624 \mathrm{E}-01$ & $5.294 \mathrm{E}-02$ & 0.381 & $6.653 \mathrm{E}-03$ & $4.719 \mathrm{E}-03$ & 288.34 \\
$7.292 \mathrm{E}-01$ & $3.460 \mathrm{E}-02$ & 0.365 & $6.364 \mathrm{E}-03$ & $4.301 \mathrm{E}-03$ & 316.33 \\
$7.412 \mathrm{E}-01$ & $4.367 \mathrm{E}-02$ & 0.371 & $6.469 \mathrm{E}-03$ & $4.455 \mathrm{E}-03$ & 305.42 \\
$7.643 \mathrm{E}-01$ & $4.170 \mathrm{E}-02$ & 0.382 & $6.670 \mathrm{E}-03$ & $4.742 \mathrm{E}-03$ & 286.93 \\
$7.457 \mathrm{E}-01$ & $3.545 \mathrm{E}-02$ & 0.373 & $6.508 \mathrm{E}-03$ & $4.511 \mathrm{E}-03$ & 301.62
\end{tabular}

Table C.3. Width of the $\mathrm{D}_{2} \mathrm{O}(100)$ peak, measured in degrees, which is converted to domain size using Eqs. (C.3) and (C.4). 


\section{Appendix D - McStas Script Used to}

\section{Simulate Instrument Performance}

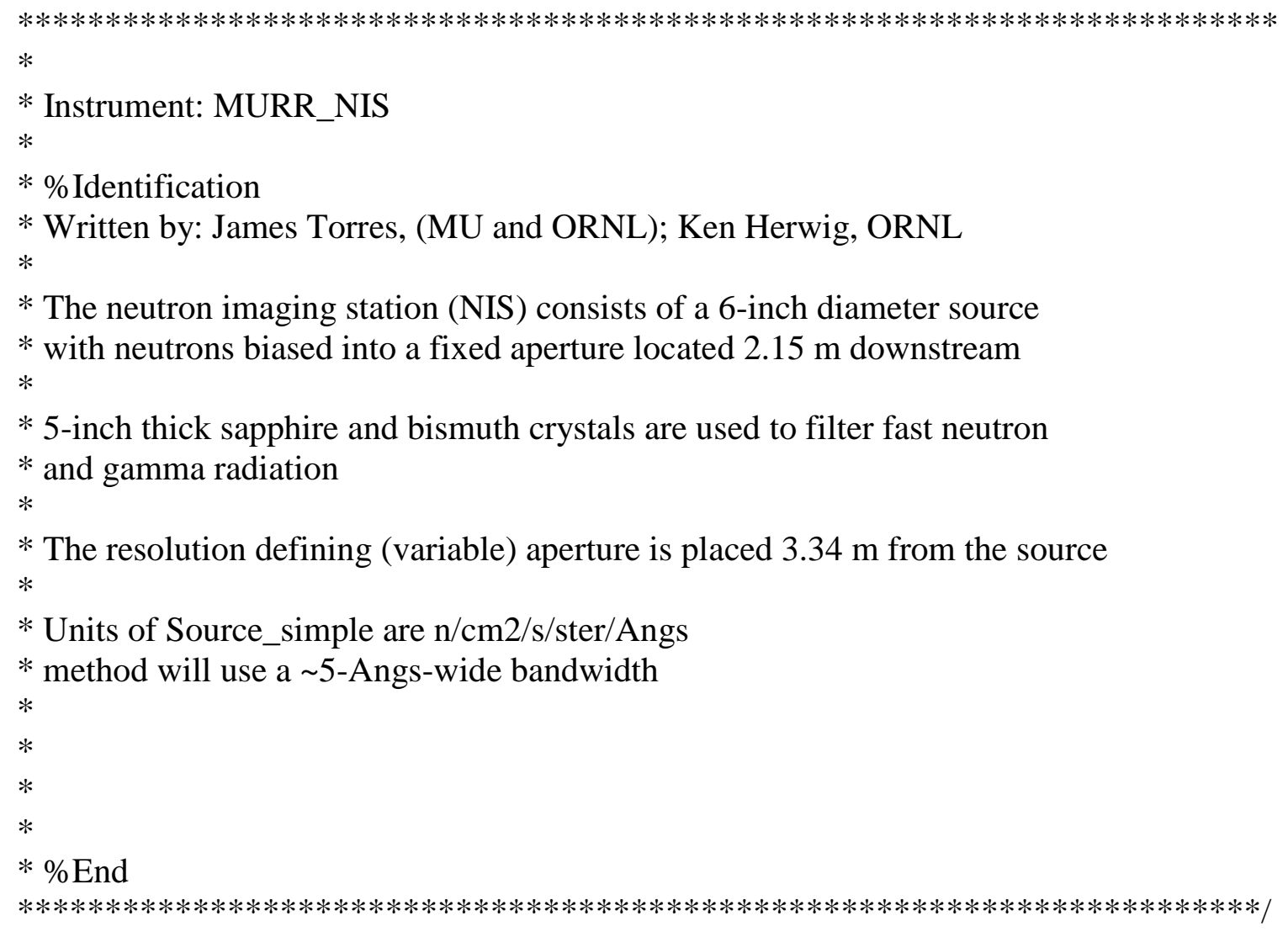

/* The DECLARE section allows us to declare variables or small */ $/ *$ functions in $\mathrm{C}$ syntax. These may be used in the whole instrument. */ DECLARE

$\%\{$

$\%\}$

/* The INITIALIZE section is executed when the simulation starts */

/* (C code). You may use them as component parameter values. */ INITIALIZE

$\%\{$

$\%\}$ 
/* Here comes the TRACE section, where the actual */

$/ *$ instrument is defined as a sequence of components. */

TRACE

COMPONENT Origin $=\operatorname{Arm}()$

AT $(0,0,0)$ ABSOLUTE

COMPONENT Progress = Progress_bar( $)$

AT $(0,0,0)$ RELATIVE Origin

/* Source focused onto the fixed aperture */

Component Moderator $=$ Source_simple $\left(\right.$ radius $=0.0762$, dist $=2.15$, focus $\_\mathrm{xw}=0.1123$, focus_yh $=0.1123$,

lambda $0=2.6$, dlambda $=2.4$ )

AT $(0,0,0)$ RELATIVE Origin

$/ *$ Fixed aperture at $2.15 \mathrm{~m}$ of $\mathrm{r}=5.615 \mathrm{~cm} * /$

Component Aperture_A = Slit(radius=0.05615)

AT $(0,0,2.15)$ RELATIVE Origin

/* Place a PSD monitor downstream from the fixed aperture */

COMPONENT FixedAp_det = PSD_monitor(

filename $=$ "FixedAp_mon.dat",

xwidth $=0.2$,

yheight $=0.2$,

restore_neutron $=1$ )

AT $(0,0,0.0001)$ RELATIVE Aperture_A

/* Place a 5-inch thick sapphire filter a distance $2.62 \mathrm{~m}$ from the source */

COMPONENT Sapphire_filter $=$ Saphire_Filter (

$\mathrm{xmin}=-0.0635$,

$\mathrm{xmax}=0.0635$,

$\mathrm{ymin}=-0.0635$,

$\mathrm{ymax}=0.0635$,

len $=0.127)$

AT $(0,0,2.62)$ RELATIVE Origin

/* Variable aperture at $3.34 \mathrm{~m}$ of $\mathrm{r}=1.25 \mathrm{~cm} *$ /

COMPONENT Aperture_B = Slit (

radius $=0.0125)$

AT $(0,0,3.34)$ RELATIVE Origin

/* Place a 5-inch thick bismuth filter $3.35 \mathrm{~m}$ from the source */

COMPONENT Bis_filter $=$ Saphire_Filter (

$\mathrm{xmin}=-0.0635$, 


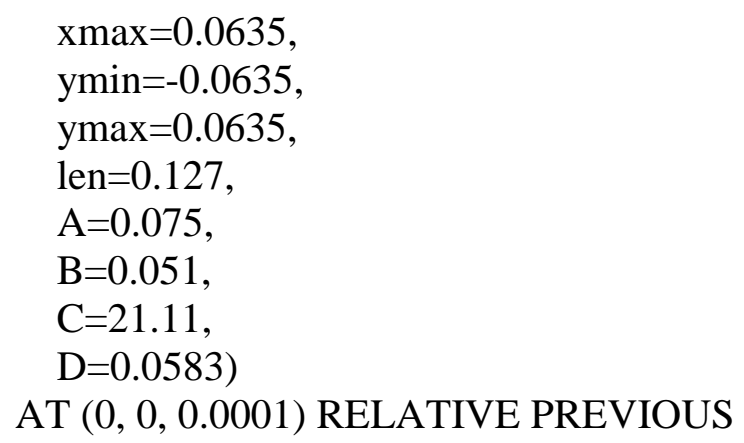

/* Now place brightness monitors at the detector plane

Component HBrightness $=$ Monitor_nD $($ xwidth $=0.5$, yheight $=0.5$, options="lambda limits $[0.2,1.8]$ bins $=250$ hdiv limits $[-2.0,2.0]$ bins $=500 "$, filename $=$ "Hdiv.dat", restore_neutron=1)

AT $(0,0,13.34)$ RELATIVE Origin

Component VBrightness $=$ Monitor_nD $($ xwidth $=0.5$, yheight $=0.5$, options="lambda limits $[0.2,1.8]$ bins $=250$ vdiv limits $[-2.0,2.0]$ bins $=500 "$, filename = "Vdiv.dat", restore_neutron=1)

AT (0,0,0.0001) RELATIVE HBrightness */

/* Wavelength monitor */

Component LambdaMonitor $=$ L_monitor(filename="Lambda.dat", xwidth $=1.0$, yheight $=1.0$,

$\mathrm{nL}=250, \operatorname{Lmin}=0.2, \operatorname{Lmax}=5.0$, restore_neutron $=1)$

AT $(0,0,13.3401)$ RELATIVE Origin

COMPONENT VariableAp_det $=$ PSD_monitor(

filename $=" M o n . d a t ", n x=1000, n y=1000$,

$\mathrm{xwidth}=1.0$,

yheight $=1.0$,

restore_neutron=1)

AT $(0,0,0.0001)$ RELATIVE PREVIOUS

COMPONENT 1_monitor = L_monitor(

$\mathrm{nL}=250$,

filename="Div.dat",

xwidth $=0.001$,

yheight $=0.001$,

$\mathrm{Lmin}=0.2$,

$\mathrm{Lmax}=5$,

restore_neutron $=1)$

AT $(0,0,0.0001)$ RELATIVE PREVIOUS

COMPONENT divergence_monitor $=$ Divergence_monitor ( $\mathrm{nh}=250$, 
$\mathrm{nv}=250$,

filename="Div_1.dat", $\mathrm{xwidth}=0.001$,

yheight $=0.001$,

maxdiv_h=0.5,

maxdiv_v $=0.5$,

restore_neutron $=1$ )

AT $(0,0,0.000001)$ RELATIVE PREVIOUS

COMPONENT Beam_stop $=$ PSD_monitor (

filename $=" B e a m S t o p . d a t ", n x=1000, n y=1000$,

$\mathrm{xwidth}=1$,

yheight $=1$,

restore_neutron $=1)$

AT $(0,0,1.31)$ RELATIVE PREVIOUS

/* This section is executed when the simulation ends ( $\mathrm{C}$ code). Other */

/* optional sections are : SAVE

*/

FINALLY

$\%\{$

$\%\}$

/* The END token marks the instrument definition end */

END 


\section{Bibliography}

1. G. E. Moore, "Cramming More Components onto Integrated Circuits," Electronics 38, No. 8 (1965).

2. G. E. Moore, "Progress in Digital Integrated Electronics," International Electron Devices Meeting, IEEE, pp. 11-13 (1975).

3. I. Mudawar, "Assessment of High-Heat-Flux Thermal Management Schemes," IEEE Transactions on Components and Packaging Technologies 24, No. 2, pp. 122-141 (2001).

4. Y. Zhang and A. Faghri, "Advances and Unsolved Issues in Pulsating Heat Pipes," Heat Transfer Engineering 29, No. 1, pp. 20-44 (2008).

5. G. Y. Eastman, “The Heat Pipe,” Scientific American 218, No. 5, pp. 38-47 (1968).

6. A. A. Abdulshaheed, P. Wang, G. Huang, and C. Li, "High Performance CopperWater Heat Pipes with Nanoengineered Evaporator Sections," J. Heat and Mass Transfer 133, pp. 474-486 (2019).

7. H. Jouhara, A. Chauhan, T. Nannou, S. Almahoud, B. Delpech, and L. C. Wrobel, "Heat Pipes Based Systems - Advances and Applications," Energy 128, pp. 729-754 (2017).

8. M. Edalatpour, L. Liu, A. M. Jacobi, K. F. Eid, and A. D. Sommers, "Managing Water on Heat Transfer Surfaces: A Critical Review of Techniques to Modify Surface Wettability for Applications with Condensation or Evaporation," Applied Energy 222, pp. 967-992 (2018).

9. Report 116-9, House Joint Resolution $1-116^{\text {th }}$ Congress, pp. 636 (2019-2020).

10. M. S. El-Genk and T. M. Schriener, "Long Operation Life Reactor for Lunar Surface Power," Nuclear Engineering and Design 241, pp. 2339-2352 (2011).

11. A. Faghri, "Review and Advances in Heat Pipe Science and Technology," J. Heat Transfer 143, 123001 (2012).

12. H. Ma, "Oscillating Heat Pipes," New York, NY: Springer (2015).

13. H. Akachi. Structure of a Heat Pipe. U.S. Patent No. 4,921,041 (1990).

14. H. Akachi. Structure of Micro-Heat Pipe. U.S. Patent No. 5,219,020 (1993). 
15. H. J. Cho, D. J. Preston, Y. Zhu, and E. N. Wang, "Nanoengineered Materials for Liquid-Vapour Phase-Change Heat Transfer," Nature Reviews 2, 16092 (2016).

16. F. Z. Zhang, R. A. Winholtz, W. J. Black, M. R. Wilson, H. Taub, and H. B. Ma, "Effect of Hydrophilic Nanostructured Cupric Oxide Surfaces on the Heat Transport Capability of a Flat-Plat Oscillating Heat Pipe," J. Heat Transfer 138, 062901 (2016).

17. J. Qu and H. Wu, "Thermal Performance Comparison of Oscillating Heat Pipes with $\mathrm{SiO}_{2}$ /water and $\mathrm{Al}_{2} \mathrm{O}_{3} /$ water Nanofluids," International Journal of Thermal Sciences 50, pp. 1954-1962 (2011).

18. H. S. Ahn, H. J. Jo, S. H. Kang, and M. H. Kim, "Effect of Liquid Spreading Due to Nano/Microstructures on the Critical Heat Flux During Pool Boiling," Appl. Phys. Lett. 98, 071908 (2011).

19. M. Ghanbarpour, N. Nikkam, R. Khodabandeh, and M. S. Toprak, "Improvement of Heat Transfer Characteristics of Cylindrical Heat Pipe by Using SiC Nanofluids," Applied Thermal Engineering 90, pp. 127-135 (2015).

20. J. Liu, X. Huang, Y. Li, K. M. Sulieman, Z. He, and F. Sun, "Hierarchical Nanostructures of Cupric Oxide on a Copper Substrate: Controllable Morphology and Wettability," J. Mater. Chem. 16, pp. 4427-4434 (2006).

21. H. B. Ma, P. Cheng, B. Borgmeyer, and Y. X. Wang, "Fluid Flow and Heat Transfer in the Evaporating Thin Film Region," Microfluid Nanofluid 4, pp. 237-243 (2008).

22. Y. Ji, H.-H. Chen, Y. J. Kim, Q. Yu, X. Ma, and H. B. Ma, "Hydrophobic Surface Effect on Heat Transfer Performance in an Oscillating Heat Pipe," J. Heat Transfer 134, 074502 (2012).

23. T. Hao, X. Ma, Z. Lan, N. Li, Y. Zhao, and H. Ma, "Effects of Hydrophilic Surface on Heat Transfer Performance and Oscillating Motion for an Oscillating Heat Pipe," International Journal of Heat and Mass Transfer 72, pp. 50-65 (2014).

24. J. Qu, X. Li, Q. Wang, F. Liu, and H. Guo, "Heat Transfer Characteristics of MicroGrooved Oscillating Heat Pipes," Experimental Thermal and Fluid Science 85, pp. 75-84 (2017).

25. D. Attinger, C. Frankiewicz, A. R. Betz, T. M. Schutzius, R. Ganguly, A. Das, C-J. Kim, and C. M. Megaridis, "Surface Engineering for Phase Change Heat Transfer: A review," MRS Energy \& Sustainability 1, pp. 1-40 (2014).

26. P. Xu and Q. Li, "Visualization Study on the Enhancement of Heat Transfer for the Groove Flat-Plate Heat Pipe with Nanoflower Coated CuO Layer," Appl. Phys. Lett. 111, 141609 (2017). 
27. "The Fukushima Daiichi Accident," Report by the Director General, Yukiya Amano, International Atomic Energy Agency (IAEA) (2015).

28. J. L. Plawsky, A. G. Fedorov, S. V. Garimella, H. B. Ma, S. C. Maroo, L. Chen, and Y. Nam, "Nano- and Microstructures for Thin-Film Evaporation - A Review," Nanoscale and Microscale Thermophysical Engineering 18, pp. 251-269 (2014).

29. T. Hao, X. Ma, Z. Lan, N. Li, and Y. Zhao, "Effects of Superhydrophobic and Superhydrophilic Surfaces on Heat Transfer and Oscillating Motion of an Oscillating Heat Pipe," J. Heat Transfer 136, 082001 (2014).

30. T. Hao, X. Ma, and Z. Lan, "Effects of Hydrophilic and Hydrophobic Surfaces on Start-Up Performance of an Oscillating Heat Pipe," J. Heat Transfer 140, 012002 (2018).

31. T. Cubaud, U. Ulmanella, and C.-M. Ho, “Two-Phase Flow in Microchannels with Surface Modifications," Fluid Dynamics Research 38, pp. $772-786$ (2006).

32. R. Chen, M-C. Lu, V. Srinivasan, Z. Wang, H. H. Cho, and A. Majumdar, "Nanowires for Enhanced Boiling Heat Transfer," Nano Letters 9, No. 2, pp. 548553 (2009).

33. T. Young, "III. An Essay on the Cohesion of Fluids," Philosophical Transactions of the Royal Society of London 95, pp. 65-87 (1805).

34. J. Drelich, E. Chibowski, D. D. Meng, and K. Terpilowski, "Hydrophilic and Superhydrophilic Surfaces and Materials," Soft Matter 7, 9804 (2011).

35. R. N. Wenzel, "Resistance of Solid Surfaces to Wetting by Water," Industrial \& Engineering Chemistry 28, No. 8, pp. 988-994 (1936).

36. A. B. D. Cassie and S. Baxter, "Wettability of Porous Surfaces," Transactions of the Faraday Society 40, pp. 546-551 (1944).

37. L. Gao and T. J. McCarthy, "How Wenzel and Cassie Were Wrong," Langmuir 23, pp. 3762-3765 (2007).

38. G. McHale, “Cassie and Wenzel: Were They Really So Wrong?” Langmuir 23, pp. 8200-8205 (2007).

39. D. Ahmad, I. van den Boogaert, J. Miller, R. Presswell, and H. Jouhara, "Hydrophilic and Hydrophobic Materials and their Applications," Energy Sources, Part A:

Recovery, Utilization, and Environmental Effects 40, No. 22, pp. 2686-2725 (2018). 
40. D. Sun and K. F. Bohringer, "Self-Cleaning: From Bio-Inspired Surface Modification to MEMS/Microfluidics System Integration,” Micromachines 10, 101 (2019).

41. S. Chen, L. Li, C. Zhao, and J. Zheng, "Surface Hydration: Principles and Applications Toward Low-Fouling/Nonfouling Biomaterials," Polymer 51, pp. 5283 -5293 (2010).

42. J. Drelich and A. Marmur, "Physics and Applications of Superhydrophobic and Superhydrophilic Surfaces and Coatings," Surface Innovations 2, No. S14, pp. 211227 (2013).

43. E. Gogolides, K. Ellinas, and A. Tserepi, "Hierarchical Micro and Nano Structured, Hydrophilic, Superhydrophobic and Superoleophobic Incorporated in Microfluidics, Microarrays and Lab on Chip Microsystems," Microelectronic Engineering 132, pp. 135-155 (2015).

44. M. Liu, S. Wang, and L. Jiang, "Nature-Inspired Superwettability Systems," Nature Reviews 2, 17036 (2017).

45. J. Yong, J. Huo, F. Chen, Q. Yang, and X. Hou, "Oil/Water Separation Based on Natural Materials with Super-Wettability: Recent Advances," Phys. Chem. Chem. Phys. 20, 25140 (2018).

46. C. J. Russo and L. A. Passmore, "Progress Towards an Optimal Specimen Support for Electron Cryomicroscopy," Current Opinion in Structural Biology 37, pp. 81-89 (2016).

47. I. Razinkov, V. P. Dandey, H. Wei, Z. Zhang, D. Melnekoff, W. J. Rice, C. Wigge, C. S. Potter, and B. Carragher, "A New Method for Vitrifying Samples for Cryo-EM," J. Structural Biology 195, pp. 190-198 (2016).

48. Y. Cai, L. Lin, Z. Xue, M. Liu, S. Wang, and L. Jiang, "Filefish-Inspired Surface Design for Anisotropic Underwater Oleophobicity," Adv. Funct. Mater. 24, pp. 809816 (2014).

49. M. Cao and L. Jiang, "Superwettability Integration: Concepts, Design and Applications," Surface Innovations 4, pp. 180-194 (2016).

50. G. E. Ewing, “Ambient Thin Film Water on Insulator Surfaces,” Chem. Rev. 106, pp. 1511-1526 (2006).

51. S. Meng, Z. Zhang, and E. Kaxiras, "Tuning Solid Surfaces from Hydrophobic to Superhydrophilic by Submonolayer Surface Modification,” PRL 97, 036107 (2006).

52. D. Quéré, "Wetting and Roughness," Ann. Rev. Mater. Res. 38, pp. 71-99 (2008). 
53. T. S. Chow, "Wetting of Rough Surfaces," J. Phys.: Condens. Matter 10, pp. L445L451 (1998).

54. T. Nishino, M. Meguro, K. Nakamae, M. Matsushita, and Y. Ueda, "The Lowest Surface Free Energy Based on - $\mathrm{CF}_{3}$ Alignment," Langmuir 15, pp. 4321-4323 (1999).

55. B. Su, Y. Tian, and L. Jiang, "Bioinspired Interfaces with Superwettability: From Materials to Chemistry," J. Am. Chem. Soc. 138, pp. 1727-1748 (2016).

56. Y. Nam and Y. S. Ju, "A Comparative Study of the Morphology and Wetting Characteristics of Micro/nanostructured Cu Surfaces for Phase Change Heat Transfer Applications," J. Adhesion Science and Technology 27, No. 20, pp. 2163-2176 (2013).

57. R. Enright, N. Miljkovic, N. Dou, Y. Nam, and E. N. Wang, "Condensation on Superhydrophobic Copper Oxide Nanostructures," J. Heat Transfer 135, 091304 (2013).

58. R. Wang, K. Hashimoto, A. Fujishima, M. Chikuni, E. Kojima, A. Kitamura, M. Shimohigoshi, and T. Watanabe, "Light-Induced Amphiphilic Surfaces," Nature 388, pp. 431-432 (1997).

59. T. A. Otitoju, A. L. Ahmad, and B. S. Ooi, "Superhydrophilic (Superwetting) Surfaces: A Review on Fabrication and Application," J. Industrial and Engineering Chemistry 47, pp. 19-40 (2017).

60. L. Zhang, N. Zhao, and J. Xu, "Fabrication and Application of Superhydrophilic Surfaces: A Review," J. Adhesion Science and Technology 28, Nos. 8-9, pp. 769-790 (2014).

61. E. Mamontov, L. Vlcek, D. J. Wesolowski, P.T. Cummings, W. Wang, L. M. Anovitz, J. Rosenqvist, C. M. Brown, and V. Garcia Sakai, "Dynamics and Structure of Hydration Water on Rutile and Cassiterite Nanopowders Studied by Quasielastic Neutron Scattering and Molecular Dynamics Simulations," J. Phys. Chem. C 111, pp. 4328-4341 (2007).

62. M.-C. Bellissent-Funel, "Protein Dynamics and Hydration Water," Water: The Forgotten Biological Molecule. Pan Stanford Publishing, Singapore, pp. 23-47 (2011).

63. S. Cerveny, F. Mallamace, J. Swenson, M. Vogel, and L. Xu, "Confined Water as Model of Supercooled Water," Chem. Rev. 116, pp. 7608-7625 (2016). 
64. W. Hagen, A. G. G. M. Tielens, and J. M. Greenberg, "A Laboratory Study of the Infrared Spectra of Interstellar Ices," Astronomy and Astrophysics Supplement Series 51, 389 (1983).

65. D. Laage, T. Elsaesser, and J. T. Haynes, "Water Dynamics in the Hydration Shells of Biomolecules," Chem. Rev. 117, pp. 10694-10725 (2017).

66. J. Swenson and S. Cerveny, "Dynamics of Deeply Supercooled Interfacial Water," J. Phys.: Condens. Matter 27, 033102 (2015).

67. C. Alba-Simionesco, B. Coasne, G. Dosseh, G. Dudziak, K. E. Gubbins, R. Radhakrishnan, and M. Sliwinska-Bartkowiak, "Effects of Confinement on Freezing and Melting," J. Phys.: Condens. Matter 18, pp. R15-R68 (2006).

68. J. B. W. Webber, "Studies of Nano-Structured Liquids in Confined Geometries and at Surfaces," Progress in Nuclear Magnetic Resonance Spectroscopy 56, pp. 78-93 (2010).

69. J. Jelassi, T. Grosz, I. Bako, M.-C. Bellissent-Funel, J. C. Dore, H. L. Castricum, and R. Sridi-Dorbez, "Structural Studies of Water in Hydrophilic and Hydrophobic Mesoporous Silicas: An X-Ray and Neutron Diffraction Study," J. Chem. Phys. 134, 064509 (2011).

70. E. B. Moore, J. T. Allen, and V. Molinero, "Liquid-Ice Coexistence Below the Melting Temperature for Water Confined in Hydrophilic and Hydrophobic Nanopores," J. Phys. Chem. C 116, pp. $7507-7514$ (2012).

71. E. B. Moore, E. de la Llave, K. Welke, D. A. Scherlis, and V. Molinero, "Freezing, Melting and Structure of Ice in a Hydrophilic Nanopore," Phys. Chem. Chem. Phys. 12, pp. 4124-4134 (2010).

72. E. G. Solveyra, E. de la Llave, D. A. Scherlis, and V. Molinero, "Melting and Crystallization of Ice in Partially Filled Nanopores," J. Phys. Chem. B 115, pp. 14196-14204 (2011).

73. D. B. Asay, A. L. Barnette, and S. H. Kim, "Effects of Surface Chemistry on Structure and Thermodynamics of Water Layers at Solid-Vapor Interfaces," J. Phys. Chem. C 113, pp. 2128-2133 (2009).

74. S. Jähnert, F. V. Chávez, G. E. Schaumann, A. Schreiber, and M. Schönhoff, and G. H. Findenegg, "Melting and Freezing of Water in Cylindrical Silica Nanopores," Phys. Chem. Chem. Phys. 10, pp. 6039-6051 (2008).

75. M. Erko, G. H. Findenegg, N. Cade, A. G. Michette, and O. Paris, "ConfinementInduced Structural Changes of Water Studied by Raman Scattering," Phys. Rev. B 84, 
104205 (2011).

76. S. Cerveny, F. Barroso-Bujans, A. Alegria, and J. Colmenero, "Dynamics of Water Intercalated in Graphite Oxide," J. Phys. Chem. C 114, pp. 2604-2612 (2010).

77. P. S. Thomas, J.-P. Guerbois, and A. Smallwood, "Low Temperature DSC Characterisation of Water in Opal," J. Therm. Anal. Calorim. 113, pp. 1255-1260 (2013).

78. G. Leone, A. Bidini, S. Lamponi, and A. Magnani, "States of Water, Surface and Rheological Characterisation of a New Biohydrogel as Articular Cartilage Substitute," Polym. Adv. Technol. 24, pp. 824-833 (2013).

79. P. G. Debenedetti and H. E. Stanley, "Supercooled and Glassy Water," Physics Today 56, No. 6, 40 (2003).

80. J.-M. Zanotti, M.-C. Bellissent-Funel, and S.-H. Chen, "Experimental Evidence of a Liquid-Liquid Transition in Interfacial Water," Europhys. Lett. 71, pp. 91-97 (2005).

81. T. Miyatou, R, Ohashi, T. Ida, S. Kittaka, and M. Mizuno, "An NMR Study on the Mechanisms of Freezing and Melting of Water in Spherically Mesoporous Silicas SBA-16," Phys. Chem. Chem. Phys. 18, 18555 (2016).

82. L. Xu, P. Kumar, S. V. Buldyrev, S.-H. Chen, P. H. Poole, F. Sciortino, and H. E. Stanley, "Relation Between the Widom Line and the Dynamic Crossover in Systems with a Liquid-Liquid Phase Transition," PNAS 102, no. 46, pp. 16558-16562 (2005).

83. P. H. Poole, F. Sciortino, U. Essmann, and H. E. Stanley, "Phase Behaviour of Metastable Water," Nature 360, pp. 324-328 (1992).

84. K. Amann-Winkel, R. Böhmer, F. Fujara, C. Gainaru, B. Geil, and T. Loerting, "Colloquium: Water's Controversial Glass Transitions," Rev. Mod. Phys. 88, no. 1, 011002 (2016).

85. L. Liu, S.-H. Chen, A. Faraone, C.-W. Yen, and C.-Y. Mou, "Pressure Dependence of Fragile-to-Strong Transition and a Possible Second Critical Point in Supercooled Confined Water," PRL 95, 117802 (2005).

86. P. G. Debenedetti, "Supercooled and Glassy Water," J. Phys.: Condens. Matter 15, pp. R1669-R1726 (2003).

87. G. H. Findenegg, S. Jähnert, D. Akcakayiran, and A. Schreiber, "Freezing and Melting of Water Confined in Silica Nanopores," ChemPhysChem 9, pp. 2651-2659 (2008). 
88. S. Cerveny, J. Colmenero, and A. Alegria, "Comment on "Pressure Dependence of Fragile-to-Strong Transition and a Possible Second Critical Point in Supercooled Confined Water,"” PRL 97, 189802 (2006).

89. J. Swenson, "Comment on "Pressure Dependence of Fragile-to-Strong Transition and a Possible Second Critical Point in Supercooled Confined Water," PRL 97, 189801 (2006).

90. S. Kittaka, S. Ishimaru, M. Kuranishi, T. Matsuda, and T. Yamaguchi, "Enthalpy and Interfacial Free Energy Changes of Water Capillary Condensed in Mesoporous Silica, MCM-41 and SBA-15," Phys. Chem. Chem. Phys. 8, pp. 3223-3231 (2006).

91. S. Kittaka, Y. Ueda, F. Fujisaki, T. Iiyama, and T. Yamaguchi, "Mechanism of Freezing of Water in Contact with Mesoporous Silicas MCM-41, SBA-15 and SBA16: Role of Boundary Water of Pore Outlets in Freezing," Phys. Chem. Chem. Phys. 13, pp. 17222-17233 (2011).

92. C. E. Bertrand, Y. Zhang, and S.-H. Chen, "Deeply-Cooled Water Under Strong Confinement: Neutron Scattering Investigations and the Liquid-Liquid Critical Point Hypothesis," Phys. Chem. Chem. Phys. 15, pp. 721-745 (2013).

93. E. Liu, J. C. Dore, J. B. W. Webber, D. Khushalani, S. Jähnert, G. H. Findenegg, and T. Hansen, "Neutron Diffraction and NMR Relaxation Studies of Structural Variation and Phase Transformations for Water/Ice in SBA-15 Silica: I. The Over-Filled Case," J. Phys.: Condens. Matter 18, pp. 10009-10028 (2006).

94. J. B. W. Webber, J. C. Dore, J. H. Strange, R. Anderson, and B. Tohidi, "Plastic Ice in Confined Geometry: The Evidence from Neutron Diffraction and NMR Relaxation," J. Phys.: Condens. Matter 19, 415117 (2007).

95. (a) V. F. Sears, "Neutron Scattering Lengths and Cross Sections," Neutron News 3, No. 3, pp. 26-37 (2006); (b) www.ncnr.nist.gov/resources/n-lengths/

96. S. Takahara, M. Nakano, S. Kittaka, Y. Kuroda, T. Mori, H. Hamano, and T. Yamaguchi, "Neutron Scattering Study on Dynamics of Water Molecules in MCM41,” J. Phys. Chem. B 103, pp. 5814-5819 (1999).

97. A. Kiwilsza, A. Pajzderska, M. A. Gonzalez, J. Mielcarek, and J. Wąsicki, "QENS and NMR Study of Water Dynamics in SBA-15 with a Low Water Content," J. Phys. Chem. C 119, pp. 16578-16586 (2015).

98. J. Liu, X. Huang, Y. Li, K. M. Sulieman, Z. He, and F. Sun, "Hierarchical Nanostructures of Cupric Oxide on a Copper Substrate: Controllable Morphology and Wettability," J. Mater. Chem. 16, pp. 4427-4434 (2006). 
99. Q. Zhang, K. Zhang, D. Xu, G. Yang, H. Huang, F. Nie, C. Liu, and S. Yang, "CuO Nanostructures: Synthesis, Characterization, Growth Mechanisms, Fundamental Properties, and Applications," Progress in Materials Science 60, pp. 208-337 (2014).

100. M. A. Dar, S. H. Nam, Y. S. Kim, and W. B. Kim, "Synthesis, Characterization, and Electrochemical Properties of Self-Assembled Leaf-Like CuO Nanostructures," J. Solid State Electrochem 14, pp. 1719-1726 (2010).

101. S. J. Pennycook, “Z-Contrast STEM for Materials Science,” Ultramicroscopy 30, pp. 58-69 (1989).

102. P. A. Midgley, M. Weyland, J. M. Thomas, and B. F. G. Johnson, “Z-Contrast Tomography: A Technique in Three-Dimensional Nanostructural Analysis Based on Rutherford Scattering," Chem. Commun. 10, 907 (2001).

103. R. F. Egerton, "Physical Principles of Electron Microscopy - An Introduction to TEM, SEM, and AEM," New York, NY, Springer (2005).

104. L. Lou, Y. Kang, Z. Liu, J. C. Yang, and G. Zhou, "Dependence of Degree of Orientation of Copper Oxide Nuclei on Oxygen Pressure During Initial Stages of Copper Oxidation," Phys. Rev. B 83, 155418 (2011).

105. G. Zhou, L. Luo, L. Li, J. Ciston, E. A. Stach, and J. C. Yang, "Step-Edge-Induced Oxide Growth During the Oxidation of Cu Surfaces," PRL 109, 235502 (2012).

106. R. F. Egerton, "Electron Energy-Loss Spectroscopy in the TEM," Rep. Prog. Phys. 72, 016502 (2009).

107. K. Sun, J. Liu, and N. D. Browning, "Direct Atomic Scale Analysis of the Distribution of $\mathrm{Cu}$ Valence States in $\mathrm{Cu} / \gamma-\mathrm{Al}_{2} \mathrm{O}_{3}$ Catalysts," Applied Catalysis B: Environmental 38, pp. 217-281 (2002).

108. G. Yang, S. Cheng, C. Li, J. Zhong, C. Ma, Z. Wang, and W. Xiang, "Investigation of the Oxidation States of $\mathrm{Cu}$ Additive in Colored Borosilicate Glasses by Electron Energy Loss Spectroscopy," J. Appl. Phys. 116, 223707 (2014).

109. A. M. Donald, "The Use of Environmental Scanning Electron Microcopy for Imaging Wet and Insulating Materials," Nature Materials 2, pp. 511-516 (2003).

110. K. Rykaczewski, J. H. J. Scott, and A. G. Fedorov, "Electron Beam Heating Effects During Environmental Scanning Electron Microscopy Imaging of Water Condensation on Superhydrophobic Surfaces," APL 98, 093106 (2011).

111. K. Rycakzewski and J. H. J. Scott, "Best Practices for Quantitative in situ ESEM Imaging of Microscale Water Condensation Dynamics on Superhydrophobic Surfaces," Microsc. Microanal. 18 (Suppl. 2), (2012). 
112. F. W. Murray, "On the Computation of Saturation Vapor Pressure," J. Appl. Meteor. 6, pp. 203-204 (1967).

113. X. Chen, J. Shu, and Q. Chen, “Abnormal Gas-Liquid-Solid Phase Transition Behaviour of Water Observed with in situ Environmental SEM," Sci. Rep. 7, 46680 (2017).

114. K. H. Kim, A. Spah, H. Pahak, F. Perakis, D. Mariedahl, K. Amann-Winkel, J. A. Sellberg, J. H. Lee, S. Kim, J. Park, K. H. Nam, T. Katayama, and A. Nilsson, "Maxima in the Thermodynamic Response and Correlation Functions of Deeply Supercooled Water," Science 358, pp. 1589-1593 (2017).

115. V. Vins, M. Fransen, J. Hykl, and J. Hruby, "Surface Tension of Supercooled Water Determined by Using a Counterpressure Capillary Rise Method," J. Phys. Chem. B 119, pp. 5567-5575 (2015).

116. X. Zhang, P. Sun, T. Yan, Y. Huang, Z. Ma, B. Zou, W. Zheng, J. Zhou, Y. Gong, and C. Q. Sun, "Water's Phase Diagram: From the Notion of Thermodynamics to Hydrogen-Bond Cooperativity," Progress in Solid State Chemistry 43, pp. 71-81 (2015).

117. E. P. Barrett, L. G. Joyner, and P. P. Halenda, "The Determination of Pore Volume and Area Distributions in Porous Substances. I. Computations from Nitrogen Isotherms," J. Am. Chem. Soc. 73, No. 1, pp. 373-380 (1951).

118. M. Thommes, K. Kaneko, A. V. Neimark, J. P. Olivier, F. Rodriguez-Reinso, J. Rouquerol, and K. S. W. Sing, "Physisorption of Gases, with Special Reference to the Evaluation of Surface Area and Pore Size Distribution (IUPAC Technical Report," Pure and Applied Chemistry 87, pp. 1051-1069 (2015).

119. S. J. Gregg and K. S. W. Sing, “Adsorption, Surface Area and Porosity,” New York, NY, Academic Press Inc. (1982).

120. V. Kruefu, A. Wisitoraat, and S. Phanichphant, "Effects of Niobium-Loading on Sulfur Dioxide Gas-Sensing Characteristics of Hydrothermally Prepared Tungsten Oxide Thick Film,” Journal of Nanomaterials 2015, 820509 (2015).

121. S. Brunauer, P. H. Emmett, and E. Teller, "Adsorption of Gases in Multimolecular Layers,” J. Am. Chem. Soc. 60, No. 2, pp. 309-319 (1938).

122. K. C. Clarke, "Computation of the Fractal Dimension of Topographic Surfaces using the Triangular Prism Surface Area Method," Computers \& Geosciences 12 , No. 5, pp. 713-722 (1986).

123. A. Meyer, R. M. Dimeo, P. M. Gehring, and D. A. Neumann, "The High-Flux Backscattering Spectrometer at the NIST Center for Neutron Research," Rev. Sci. Instrum. 74, 2759 (2003).

124. E. Mamontov and K. W. Herwig, "A Time-of-Flight Backscattering Spectrometer at the Spallation Neutron Source, BASIS," Rev. Sci. Instrum. 82, 085109 (2011). 
125. K. W. Herwig and W. S. Keener, "Design and Performance of the Spallation Neutron Source Backscattering Spectrometer," Appl. Phys. A $\mathbf{7 4}$ [Suppl.], pp. S1592-S1594 (2002).

126. J. Jelassi, H. L. Castricum, M.-C. Bellissent-Funel, J. Dore, J. B. W. Webber, and R. Sridi-Dorbez, "Studies of Water and Ice in Hydrophilic and Hydrophobic Mesoporous Silicas: Pore Characterisation and Phase Transitions," Phys. Chem. Chem. Phys. 12, 2838 (2010).

127. E. Mamontov, Y, Yue, J. Bahadur, J. Guo, C. I. Contescu, N. C. Gallego, and Y. B. Melnichenko, "Hydration Level Dependence of the Microscopic Dynamics of Water Adsorbed in Ultramicroporous Carbon," Carbon 111, pp. 705-712 (2017).

128. E. J. Kintzel Jr., M. K. Kidder, A. C. Buchanan III, P. F. Britt, E. Mamontov, M. Zamponi, and K. W. Herwig, "Dynamics of 1,3-Diphenylpropane Tethered to the Interior Pore Surfaces of MCM-41," J. Phys. Chem. C 116, pp. 923-932 (2012).

129. A. Miskowiec, Z. N. Buck, F. Y. Hansen, H. Kaiser, H. Taub, M. Tyagi, S. O. Diallo, E. Mamontov, and K. W. Herwig, "On the Structure and Dynamics of Water Associated with Single-Supported Zwitterionic and Anionic Membranes," J. Chem. Phys. 147, 125102 (2017).

130. A. Miskowiec, Z. N. Buck, M. C. Brown, H. Kaiser, F. Y. Hansen, G. M. King, H. Taub, R. Jiji, J. W. Cooley, M. Tyagi, S. O. Diallo, E. Mamontov, and K. W. Herwig, "On the Freezing Behavior and Diffusion of Water in Proximity to SingleSupported Zwitterionic and Anionic Bilayer Lipid Membranes," EPL 107, 28008 (2014).

131. L. Liu, A. Faraone, C.-Y. Mou, C.-W. Yen, and S.-H. Chen, "Slow Dynamics of Supercooled Water Confined in Nanoporous Silica Materials," J. Phys.: Condens. Matter 16, pp. S5403-S5436 (2004).

132. M. A. Henderson, "The Interaction of Water with Solid Surfaces: Fundamental Aspects Revisited," Surface Science Reports 46, pp. 1-308 (2002).

133. E. G. Solveyra, E. de la Llave, D. A. Scherlis, and V. Molinero, "Melting and Crystallization of Ice in Partially Filled Nanopores," J. Phys. Chem. B 115, pp. 14196-14204 (2011).

134. J. Seyed-Yazdi, J. C. Dore, J. B. W. Webber, and H. Farman, "Structural Characterization of Water and Ice in Mesoporous SBA-15 silicas IV: Partially Filled Cases for 86 Å Pore Diameter," J. Phys.: Condens. Matter 25, 465015 (2013).

135. R. Berliner, D. F. R. Mildner, J. Sudol, and H. Taub, "Position Sensitive Detectors and Data Collection Systems at the University of Missouri Research Reactor Facility," Position Sensitive Detection of Thermal Neutrons (Academic, New York), pp. 120 (1983). 
136. K. Röttger, A. Endriss, J. Ihringer, S. Doyle, and W. F. Kuhs, "Lattice Constants and Thermal Expansion of $\mathrm{H}_{2} \mathrm{O}$ and $\mathrm{D}_{2} \mathrm{O}$ Ice Ih Between 10 and $265 \mathrm{~K}$," Acta. Cryst. B50, pp. 644-648 (1994).

137. Z. N. Buck, "Neutron Scattering Studies of Water Diffusion Near the Interface of Model Cell Membranes," PhD Dissertation, University of Missouri, 2018.

138. B. D. Cullity, “Elements of X-Ray Diffraction,” Reading, Massachusetts: AddisonWesley, 1978.

139. P. A. Korzhavyi and B. Johansson, "Literature Review on the Properties of Cuprous Oxide $\mathrm{Cu}_{2} \mathrm{O}$ and the Process of Copper Oxidation," Report (SKB-TR-11-08) Sweden, (2011).

140. J. B. Forsyth and S. Hull, "The Effect of Hydrostatic Pressure on the Ambient Temperature Structure of CuO," J. Phys.: Condens. Matter 3, pp. 5257-5261 (1991).

141. S. F. Parker, A. J. Ramirez-Cuesta, and L. Daemen, "Vibrational Spectroscopy with Neutrons: Recent Developments," Spectrochimica Acta Part A: Molecular and Biomolecular Spectroscopy 190, pp. 518-523 (2018).

142. Y. Edura and N. Morishima, "Cold and Thermal Neutron Scattering in Liquid Water: Cross-Section Model and Dynamics of Water Molecules," Nuclear Instruments and Methods in Physics Research A 534, pp. 531-543 (2004).

143. J. Li, "Inelastic Neutron Scattering Studies of Hydrogen Bonding in Ices," J. Chem. Phys. 105, 6733 (1996).

144. E. C. Spencer, N. L. Ross, S. F. Parker, R. E. Olsen, and B. F. Woodfield, "Inelastic Neutron Scattering Studies of Hydrated $\mathrm{CuO}, \mathrm{ZnO}$ and $\mathrm{CeO}_{2}$," Chemical Physics 427, pp. 66-70 (2013).

145. S. Kittaka, S. Takahara, H. Matsumoto, Y. Wada, T. J. Satoh, and T. Yamaguchi, "Low Temperature Phase Properties of Water Confined in Mesoporous Silica MCM-41: Thermodynamic and Neutron Scattering Study," J. Chem. Phys. 138, 204714 (2013).

146. A. Paciaroni, A. Orecchini, G. Goracci, E. Cornicchi, C. Petrillo, and F. Sacchetti, "Glassy Character of DNA Hydration Water," J. Phys. Chem. B 117, pp. 20262031 (2013).

147. I. Michalarias, I. Beta, R. Ford, S. Ruffle, and J. Li, "Inelastic Neutron Scattering Studies of Water in DNA," Applied Physics A 74, pp. S1242-S1244 (2002).

148. R. C. Ford, S. V. Ruffle, A. J. Ramirez-Cuesta, I. Machalarias, I. Beta, A. Miller, and J. Li, "Inelastic Incoherent Neutron Scattering Measurements of Intact Cells and Tissues and Detection of Interfacial Water," J. Am. Chem. Soc. 126, no. 14, pp. 4682-4688 (2004). 
149. E. A. Engel, A. Anelli, M. Ceriotti, C. J. Pickard, and R. J. Needs, "Mapping Uncharted Territory in Ice from Zeolite Networks to Ice Structures," Nature Communications 9, pp. 2173 (2018).

150. C. A. Angell, “Amorphous Water,” Annu. Rev. Phys. Chem. 55, pp. 559-583 (2004).

151. T. Loerting and N. Giovambattista, "Amorphous Ices: Experiments and Numerical Simulations," J. Phys.: Condens. Matter 18, pp. R919-R977 (2006).

152. T. Loerting, K. Winkel, M. Seidl, M. Bauer, C. Mitterdorfer, P. H. Handle, C. G. Salzmann, E. Mayer, J. L. Finney, and D. T. Bowron, "How Many Amorphous Ices are There?” Phys. Chem. Chem. Phys. 13, pp. 8783-8794 (2011).

153. E. F. Burton and W. F. Oliver, "X-ray Diffraction Patterns of Ice," Nature 135, no. 3413, pp. 505 (1935).

154. P. Brüggeller and E. Mayer, "Complete Vitrification in Pure Liquid Water and Dilute Aqueous Solutions," Nature 288, no. 5791, pp. 569 (1980).

155. E. Mayer and R. Pletzer, "Polymorphism in Vapor Deposited Amorphous Solid Water," J. Chem. Phys. 80, no. 6, pp. 2939-2952 (1984).

156. C. A. Tulk, J. J. Molaison, A. R. Makhluf, C. E. Manning, and D. D. King, "Absence of Amorphous Forms When Ice is Compressed at Low Temperature," Nature 569, no. 7757, pp. 542 (2019).

157. D. T. Bowron, J. L Finney, A. Hallbrucker, I. Kohl, T. Loerting, E. Mayer, and A. K. Soper, "The Local and Intermediate Range Structures of the Five Amorphous Ices at $80 \mathrm{~K}$ and Ambient Pressure: A Faber-Ziman and Bhatia-Thornton Analysis," J. Chem. Phys. 125, 194502 (2006).

158. Z. N. Buck, J. Torres, A. Miskowiec, E. Mamontov, H. Kaiser, F. Y. Hansen, H. Taub, M. Tyagi, L. Collins, and K. W. Herwig, "Effect of Melittin on Water Diffusion and Membrane Structure in DMPC Lipid Bilayers," EPL 123, 18002 (2018).

159. R. T. Azuah, L. R. Kneller, Y. Qiu, P. L. W. Tregenna-Piggott, C. M. Brown, J. R. D. Copley, and R. M. Dimeo, "DAVE: A Comprehensive Software Suite for the Reduction, Visualization, and Analysis of Low Energy Neutron Spectroscopic Data," J. Res. Natl. Inst. Stan. Technol. 114, 341 (2009).

160. M. Bée, “Quasielastic Neutron Scattering: Principles and Applications in Solid State Chemistry, Biology, and Materials Science," Hilger, A.: Philadelphia, PA, 1988; pp. 150-153.

161. J. Teixeira, M.-C. Bellissent-Funel, S. H. Chen, and A. J. Dianoux, "Experimental Determination of the Nature of Diffusive Motions of Water Molecules at Low Temperatures," Phys. Rev. A 31, No. 3, pp. 1913-1917 (1985).

162. D. Di Cola, A. Deriu, M. Sampoli, and A. Torcini, "Proton Dynamics in Supercooled Water by Molecular Dynamics Simulations and Quasielastic Neutron Scattering," J. Chem. Phys. 104, no. 11, pp. 4223-4232 (1996). 
163. J. Qvist, H. Schober, and B. Halle, "Structural Dynamics of Supercooled Water from Quasielastic Neutron Scattering and Molecular Simulations," J. Chem. Phys. 134, 144508 (2011).

164. F. Cavatorta, A. Deriu, D. Di Cola, and H. D. Middendorf, "Diffusive Properties of Water Studied by Incoherent Quasi-Elastic Neutron Scattering," J. Phys.: Condens. Matter 6, pp. A113-A117 (1994).

165. J.-M. Zanotti, G. Gibrat, and M.-C. Bellissent-Funel, "Hydration Water Rotational Motion as a Source of Configurational Entropy Driving Protein Dynamics. Crossovers at 150 and 220 K," Phys. Chem. Chem. Phys. 10, pp. 48654870 (2008).

166. K. Yoshida, T. Yamaguchi, S. Kittaka, M.-C. Bellissent-Funel, and P. Fouquet, "Thermodynamic, Structural, and Dynamic Properties of Supercooled Water Confined in Mesoporous MCM-41 Studied with Calorimetric, Neutron Diffraction, and Neutron Spin-Echo Measurements," J. Chem. Phys. 129, 054702 (2008).

167. S.-K. Wang, E. Mamontov, M. Bai, F. Y. Hansen, H. Taub, J. R. D. Copley, V. García-Sakai, G. Gasparovic, T. Jenkins, M. Tyagi, K. W. Herwig, D. A. Neumann, W. Montfrooij, and U. G. Volkmann, "Localized Diffusive Motion on Two Different Time Scales in Solid Alkane Nanoparticles," EPL 91, 66007 (2010).

168. J. Torres, Z. N. Buck, H. Kaiser, X. He, T. White, M. Tyagi, R. A. Winholtz, F. Y. Hansen, K. W. Herwig, and H. Taub, "A Neutron Scattering and Electron Microscopy Study of the Structure, Wetting, and Freezing Behavior of Water Near Hydrophilic CuO-Nanostructured Surfaces,” J. Appl. Phys. 125, 025302 (2019).

169. S. ZhaoRu, S. Gang, C. YiXuan, and Xu LiMei, "Liquid-Liquid Phase Transition in Water," Sci. China-Phys. Mech. Astron. 57, pp. 810-818 (2014).

170. L. Santodonato, H. Bilheux, B. Bailey, J. Bilheux, P. Nguyen, A. Tremsin, D. Selby, and L. Walker, "The CG-1D Neutron Imaging Beamline at the Oak Ridge National Laboratory High Flux Isotope Reactor,” Physics Procedia 69, pp. 104-108 (2015).

171. J. Huotari, "Reactor Shutdown Affects Experiments, Isotope Production at HFIR," Oak Ridge Today, 29 November 2018.

172. C. Wilson, B. Borgmeyer, R. A. Winholtz, H. B. Ma, D. L. Jacobson, D. S. Hussey, and M. Arif, "Visual Observation of Oscillating Heat Pipes Using Neutron Radiography," J. Thermophysics and Heat Transfer 22, No. 3, pp. 366-372 (2008).

173. H. B. Ma, B. Borgmeyer, P. Cheng, and Y. Zhang, "Heat Transport Capability in an Oscillating Heat Pipe," J. Heat Transfer 130, 081501 (2008).

174. J. Torres, Z. N. Buck, H. Kaiser, M. Tyagi, F. Y. Hansen, K. W. Herwig, E. Mamontov, L. Daemen, M. K. Kidder, and H. Taub, "Study of the Water Dynamics Near Hydrophilic Nanostructured CuO Surfaces by Inelastic Neutron Scattering," in preparation.

175. S. Stover, "Thermal Performance of a Nam-Ju Nanostructured Copper Oscillating Heat Pipe," M.S. Thesis, University of Missouri, 2018. 
176. C. A. Schneider, W. S. Rasband, and K. W. Eliceiri, "NIH Image to ImageJ: 25 Years of Image Analysis," Nature Methods 9, No. 7, pp. 671-675 (2012).

177. T. Wang, F. Li, S. R. Parnell, W. A. Hamilton, H. Kaiser, A. L. Washington, D. V. Baxter, and R. Pynn, "Neutron Spin Manipulation Devices using YBCO Films," Journal of Physics: Conference Series 528, No. 1 (2014).

178. C. A. Jeffrey, E. H. Conrad, R. Feng, M. Hupalo, C. Kim, P. J. Ryan, P. F. Miceli, and M. C. Tringides, "Influence of Quantum Size Effects on Island Coarsening," Phys. Rev. Lett. 96, 106105 (2006).

179. O. D. Osborne, A. Pring, R. S. Popelka-Filcoff, J. W. Bennett, A. Stopic, M. D. Glascock, and C. E. Lenehan, "Comparison of the Relative Comparator and k0 Neutron Activation Analysis Techniques for the Determination of Trace-Element Concentrations in Pyrite," Mineralogical Magazine 76, No. 5, pp. 1229-1245 (2012).

180. "University of Missouri Research Reactor First U.S. I-131 Supplier in 30 Years," Imaging Technology News, published 13 November 2018, accessed 20 April 2019. (url: https://www.itnonline.com/content/university-missouri-research-reactor-firstus-i-131-supplier-30-years).

181. "Four US Companies Chosen for Mo-99 Production Funding," World Nuclear News, published 21 February 2019, accessed 20 April 2019 (url: http://worldnuclear-news.org/Articles/Four-US-companies-chosen-for-Mo-99-productionfund).

182. P. J. Kueffer, C. A. Maitz, A. A. Khan, S. A. Schuster, N. I. Shlyakhtina, S. S. Jalisatgi, J. D. Brockman, D. W. Nigg, and M. F. Hawthorne, "Boron Neutron Capture Therapy Demonstrated in Mice Bearing EMT6 Tumors Following Selective Delivery of Boron by Rationally Designed Liposomes," Proceedings of the National Academy of Sciences of the United States of America 110, No. 16, 6512 (2013).

183. M. T. Boulanger, B. Buchanan, M. J. O’Brien, B. G. Redmond, M. D. Glascock, and M. I. Eren, "Neutron Activation Analysis of 12,900-year-old Stone Artifacts Confirms 450-510+ Clovis Tool-stone Acquisition at Paleo Crossing (33ME274), Northeast Ohio, U.S.A.," Journal of Archaeological Science 53, pp. 550-558 (2015).

184. J. M. Osborn, K. J. Glennon, E. D. Kitcher, J. D. Burns, C. M. Folden III, S. S. Chirayath, "Computation and Experimental Forensics Characterization of Weapons-Grade Plutonium Produced in a Thermal Neutron Environment," Nuclear Engineering and Technology 50, pp. 820-828 (2018).

185. S. B. Wright, "Neutron Fluence Measurements, Ch. 2: Neutron Spectra," Technical Reports Series No. 107, International Atomic Energy Agency (IAEA), Vienna 
(1970).

186. R. M. Brugger, "The University of Missouri Research Reactor, MURR,” Use and Development of Low and Medium Flux Research Reactors, Proc. International Symposium on the Use and Development of Low and Medium flux Research Reactors, pp. 166-173, Massachusetts Institute of Technology, Cambridge, MA, USA (1983).

187. R. M. Brugger, J.-L. A Shih, and H. B. Liu, “An Epithermal Neutron Beam for Neutron Capture Therapy at the Missouri University Research Reactor," Nuclear Technology 98, No. 3, pp. 322-332 (1992).

188. J. Brockmann, D. W. Nigg, M. F. Hawthorne, and C. McKibben, "A New SingleCrystal Thermal Neutron Source for Neutron Capture Therapy Research at the University of Missouri," International Conference on Reactor Physics, Nuclear Power: A Sustainable Resource, Casino-Kursaal Conference Center, Interlaken, Switzerland, Sept. 14-19 (2008).

189. F. Gullmeier, private communication, June 2019

190. H. Bilheux, K. Herwig, S. Keener, and L. Davis, "Overview of the Conceptual Design of the Future VENUS Neutron Imaging Beam Line at the Spallation Neutron Source," Physics Procedia 69, pp. 55-59 (2015).

191. D. S. Hussey, D. L. Jacobson, M. Arif, P. R. Huffman, R. E. Williams, and J. C. Cook, "New Neutron Imaging Facility at the NIST," Nucl. Instrum. Meth. Phys. Res. A 542, pp. 9-15 (2005).

192. F. Grünauer, "Design, Optimization, and Implementation of the New Neutron Radiography Facility at FRM-II," PhD Dissertation, Technische Universität München (2005).

193. E. Calzada, F. Gruenauer, M. Mühlbauer, B. Schillinger, and M. Schulz, "New Design for the ANTARES-II Facility for Neutron Imaging at FRM II," Nucl. Instrum. Meth. Phys. Res. A 605, pp. 50-53 (2009).

194. P. von der Hardt and H. Röttger, "Neutron Radiography Handbook: Nuclear Science and Technology," Springer Science and Business Media (2012).

195. A. K. Freund, "Cross-Sections of Materials Used as Neutron Monochromators and Filters," Nucl. Instrum. Methods 213, pp. 495-501 (1983).

196. (a) N. Soppera, M. Bossant, E. Dupont, "JANIS 4: An Improved Version of the NEA Java-based Nuclear Data Information System", Nuclear Data Sheets, Volume 120, June 2014, Pages 294-296. (b) http://www.oecd-nea.org/janisweb/ 
197. C. Subramanian, A. K. Suri, and T. S. R. Ch. Murthy, "Development of BoronBased Materials for Nuclear Applications," Barc Newsletter 313, pp. 14 - 22 (2010).

198. F. Piscitelli, "Boron-10 Layers, Neutron Reflectometry and Thermal Neutron Gaseous Detectors," PhD Thesis, University of Perugia (2013).

199. A. K. Suri, C. Subramanian, J. K. Sonber, and T. S. R. Ch. Murthy, "Synthesis and Consolidation of Boron Carbide: A Review," International Materials Reviews 55, pp. 4-40 (2010).

200. Cadmium, ESPI Metals (https://www.espimetals.com/index.php/online-catalog/346Cadmium), accessed 23 May 2019.

201. P. Rinard, "Neutron Interactions with Matter," Passive Nondestructive Assay of Nuclear Materials, pp. 357-377 (1991).

202. J. D. Cossairt, "Radiation Physics for Personnel and Environmental Protection," Ch. 6, Fermilab Report TM-1834, Revision 9B, Fermi National Accelerator Laboratory (2007).

203. N. M. Schaeffer, "Reactor Shielding for Nuclear Engineers," No. TID-25951, Radiation Research Associates, Fort Worth, TX (1973).

204. D. F. R. Mildner and G. P. Lamaze, "Neutron Transmission of Single-Crystal Sapphire,” J. Appl. Cryst. 31, pp. 835-840 (1998).

205. G. K. White, “Thermal Expansion of Reference Materials: Copper, Silica and Silicon," J. Appl. Phys. D 6, no. 17, pp. 2070 (1973).

206. I. B. Krynetskiı̌, B. A. Gizhevskiı̌, S. V. Naumov, and E. A. Kozlov, "Size Effect of the Thermal Expansion of Nanostructural Copper Oxide," Physics of the Solid State 50, no. 4, pp 756-758 (2008). 


\section{VITA}

James Torres was born in Phoenix, Arizona and later relocated to New Jersey where he spent most of his childhood. In May of 2014, James graduated from Rowan University with a Bachelor of Science degree in Physics, and in the following summer he participated in a research fellowship at the National Institute of Standards and Technology where he was introduced to neutron scattering. James began his graduate work in the Fall of 2014 at the University of Missouri - Columbia under the supervision of Prof. Haskell Taub. Between 2017 and 2019, he participated in an Oak Ridge National Laboratory-funded Graduate Opportunity (GO!) program during which time he conducted a large portion of his PhD research. 